PhD Dissertation

\title{
Enhanced fluid characterization in the millimeter-wave band using Gap Waveguide Technology
}

\author{
Universitat Politècnica de València \\ Colaboración con TELECOM BRETAGNE (FRANCIA) \\ Departamento de Comunicaciones
}

Author

Cristina Arenas Buendia

Advisor

Alejandro Valero Nogeuira

Valencia, January 2015 

Acknowledgments

A mi familia 


\section{ACKNOWLEDGMENTS}

During the three years of my $\mathrm{PhD}$ thesis, I have had the opportunity to learn and gain meaningful and valuable work experience thanks to Télécom Bretagne and the Universitat Politècnica de València.

I am grateful to Philippe Ferrari, Serge Verdeyme and Jose Luis Vazquez Roy for reviewing my thesis and I appreciate their interest in my work as well as their insightful comments.

I thank Eric Rius and Mariano Baquero for participating in the PhD jury and for the interest they showed in my work.

My PhD advisers played a key role in achieving this work and I am very grateful for their supervision and support:

Je voudrais remercier à François Gallée pour l'encadrement et pour toute l'aide apporté pendant ces trois années de thèse. Aussi je voudrais remercier à mon directeur de thèse Christian Person pour m'avoir guidé pendant ces années et pour ses commentaires.

También me gustaría agradecer a Alejandro Valero, mi director de la UPV, por su ayuda y por siempre estar ahí cuando se le necesita.

Je tiens à remercier également au département de micro-ondes de Télécom Bretagne, à toute l'équipe LTCC, spécialement à Camilla Karnfelt pour toute 
l'aide dans le laboratoire. Merci beaucoup à Maïna aussi pour l'aide en fabrication.

Je tiens à saluer les doctorants du département que j'ai eu le plaisir de rencontrer pendant cette thèse et remercier à Sandra et Véronique pour les bonnes moments passés dans le département.

También agradacer a todo el GRE su ayuda y gracias por los buenos momentos pasados en el grupo, el primer año y todas las veces que he estado alli han sido muy especiales. Muchas gracias a todos los profesores y gracias al equipo GRE y en general del iTEAM: Nora, Maria, Alicia, Rocio, Dani, Miguel, Toni, Tomy, Dani, Toni B, Fernando, Diana y todos los demás. Muchas gracias también Javi por haberme ayudado y haber estado ahí en algunos momentos difíciles de la tesis.

También me gustaria agradecer a mis amigos de Télecom : Dani, Daniel, Juan David, Javi, Ronald, etc. Y a todos mis amigos de Brest y a la comunidad hispanobrestois con los que pude pasar muy buenos momentos: Isa, Santi, July, Patri, Luiz, Encarna, Miguel, Soraya, etc.

No podría olvidar a mis amigos de Valencia, de Sedavi, de la Universidad y en especial mis chicas de volley, cada vez que estoy en Valencia me motiváis y me animáis a seguir con todo.

Finalement, je voudrais remercier Adrien qui à été mon soutien le plus important, en particulier pendant la rédaction et à la fin de la thèse. Tu as été une grande motivation. Je n'ai pas de mots pour te remercier pour tout ce que tu as fait et tout ce que tu fais toujours. Merci.

Y para acabar a mi familia, que siempre ha estado ahí, y si he conseguido acabar esta tesis y estar donde estoy es gracias a ellos, gracias mamá, papá y a mi hermano por estar ahí siempre. 


\section{ABSTRACT (ENGLISH)}

Microfluidic systems have emerged as a promising technology for molecular analysis, biodefence and microelectronics. Fluid sensing by microwave sensors based on the RF analysis offers new possibilities for the characterization of mediums by non-invasive methods. Several techniques are available in the frequency domain for analyzing the dielectric properties of liquids and their composition. Among them, one of the most accurate techniques is the resonant cavity methods. However, these techniques are incompatible with lab-on-chip process due its dimensions in the millimeter-wave band $(\lambda<1 \mathrm{~cm})$. In this context, a new structure called gap waveguide appears as a good candidate to overcome the principal drawbacks of the classical resonant cavities. This thesis presents the development of the gap waveguide technology in the millimeter-wave band. Other conventional technologies are discussed as well, to compare them with the performance in terms of gap waveguide losses. We also present the resonator design based on gap waveguide capable of working in the microfluidic sensing domain in the millimeter-wave band. In this context, I present a comparative study between gap waveguide and Substrate Integrated Cavity (SIC) with the aim to characterize the fluid permittivity at $60 \mathrm{GHz}$. With this purpose, several prototypes have been manufactured with Printed Circuit Board (PCB) and Low Temperature Co-fired Ceramic (LTCC) technologies. An important experimental was carried out to validate work some critical steps in the LTCC process which are important for the gap waveguide manufacturing, particularly those related with the creation of cavities using LTCC materials. 
Key words: Microfluidic, Millimeter-wave band, Gap Waveguide, Low Temperature Co-fired Ceramic (LTCC), Printed Circuit Board (PCB), Substrate Integrated Waveguide (SIW) 


\section{RÉSUMÉ}

Les systèmes micro-fluidiques apparaissent comme une technologie très prometteuse pour l'analyse moléculaire, la bio-défense et la micro-électronique. La détection de fluides par des capteurs à micro-ondes fondée sur l'analyse $\mathrm{RF}$ offre de nouvelles possibilités pour la caractérisation des milieux par des méthodes non invasives. Plusieurs techniques RF existent pour analyser les propriétés diélectriques des liquides et leur composition. Parmi elles, celle qui a été choisie car étant l'une des plus précises repose sur cavités résonants. Toutefois, la plupart de ces techniques ne sont pas compatibles avec le processus " lab-on-chip " en raison de leurs faibles dimensions dans la bande des fréquences millimétriques $(\lambda<1 \mathrm{~cm})$. Dans ce contexte, une nouvelle structure appelée « gap waveguide" apparaît comme être un bon candidat pour pallier les principaux inconvénients des techniques classiques. Cette thèse présente le développement de la technologie "gap waveguide" dans la bande millimétrique. Elle est comparée à d'autres technologies classiques telle la technologie SIW (" Substrate Integrated Waveguide ») afin d'évaluer ses performances en termes de pertes. Par la suite, je présente la conception du résonateur basée sur "gap waveguide» compatible avec la création d'un canal micro-fluidique dans la bande millimétrique. Dans ce cadre, j’ai effectué une étude comparative entre la « gap waveguide " et "Substrate Integrated Cavity " afin de caractériser la permittivité des fluides à $60 \mathrm{GHz}$. Pour ce faire, plusieurs prototypes ont été fabriqués avec les technologies «Printed Circuit Board (PCB)» et Low Temperature Co-fired Ceramic (LTCC)». Un important travail expérimental a été réalisé pour valider 
les étapes critiques du procédé LTCC avec la technologie "gap Waveguide ", en particulier celles liées à la création de cavités.

Mots-clés: Microfluidique, Bande milimétrique, Gap Waveguide, Low Temperature Co-fired Ceramic (LTCC), Printed Circuit Board (PCB), Substrate Integrated Waveguide (SIW) 


\section{RESUMEN}

Los sistemas micro-fluidicos han emergido como una tecnología prometedora para el análisis molecular, la bio-defensa y la micro-electrónica. La detección de fluidos por sensores de microondas basada en análisis $\mathrm{RF}$, ofrece nuevas posibilidades para la caracterización de medios a través de métodos no-invasivos. Diferentes técnicas son disponibles en el dominio frecuencial para analizar las propiedades dieléctricas de los líquidos y su composición. Entre ellas, una de las técnicas más precisas son los métodos de cavidad resonantes. Sin embargo, estas técnicas son incompatibles con los procesos "lab־on-chip» debidos a sus dimensiones en la banda de frecuencias milimétricas $(\lambda<1 \mathrm{~cm})$. En este contexto, una nueva estructura denominada "gap waveguide" aparece como un buen candidato para superar los principales problemas de las clásicas cavidades resonantes. Esta tesis presenta el desarrollo de la tecnología "gap waveguide" en la banda de frecuencias milimétricas. Otras tecnologías serán analizadas también, para compararlas en términos de propiedades y pérdidas con la "gap waveguide». También se presentara el diseño del resonador basado en la topología «gap waveguide " capaz de trabajar en el dominio de la detección de microfluidos en la banda de ondas milimétricas. En este contexto, presento un estudio comparativo entre la " gap waveguide " y « Substrate Integrated Cavity (SIC) » con el objetivo de caracterizar fluidos a $60 \mathrm{GHz}$. Con este objetivo, varios prototipos fueron fabricados con la tecnología de circuitos impresos «PCB» y con LTCC «Low Temperature Co-fired Ceramic». Un importante trabajo experimental para 
validar algunas etapas críticas en el proceso LTCC, que son importantes para la fabricación de la "gap waveguide, en particular las relacionadas con la creación de cavidades utilizando materiales LTCC.

Palabras clave: Micro-fluidica, banda de frecuencias milimétricas, Gap Waveguide, Low Temperature Co-fired Ceramic (LTCC), Printed Circuit Board (PCB), Substrate Integrated Waveguide (SIW) 


\section{RESUM}

Els sistemes microfluidics s'han convertit en una tecnologia prometedora per a l'anàlisi molecular i la microelectrònica, així com també el combat contra el bioterrorisme. La detecció de fluïts per sensors de microones (basada en l'anàlisi RF) ofereix noves possibilitats per a la caracterització de medis a través de mètodes no invasius. Diferents tècniques són disponibles en el domini freqüencial per a analitzar la composició i les propietats dielèctriques dels fluïts. Una de les tècniques més precises és el mètode anomenat de cavitats ressonants. No obstant, aquestes tècniques són incompatibles amb els processos lab-on-chip degut a les dimensions físiques requerides per poder ser utilitzades en banda de freqüències mil limètriques $(\lambda<1 \mathrm{~cm})$. En aquest context, una nova estructura denominada gap waveguide es presenta com una bona candidata per a superar els principals problemes lligats a les clàssiques cavitats ressonants. Aquesta tesi tracta doncs del desenvolupament de la tecnologia gap waveguide en la banda de freqüències mil limètriques. Altres tecnologies seran analitzades per a comparant-les en termes de propietats i pèrdues. Es presentarà també el disseny del ressonador basat en la topologia gap waveguide per a la detecció de microfluïts en la banda d'ones mil limètriques. En el marc d'aquesta tesi, es presenta també un estudi comparatiu entre la gap waveguide i Substrate Integrated Cavity (SIC) amb l'objectiu de caracteritzar fluïts a $60 \mathrm{GHz}$. Amb aquest objectiu, diversos prototips han estat fabricats amb la tecnologia de circuits impresos (PCB) i amb Low Temperature Co-fired Ceramic (LTCC). Un important treball experimental ha estat realitzat per a validar algunes etapes crítiques durant el procés LTCC. 
Aquestes etapes són importants per a la fabricació de la gap waveguide, en particular aquelles relacionades amb la creació de cavitats utilitzant materials LTCC.

Paraules clau: Microfluïts, banda de freqüències mil limètriques, Gap Waveguide, Low Temperature Co-fired Ceramic (LTCC), Printed Circuit Board (PCB), Substrate Integrated Waveguide (SIW) 


\section{TABLE OF CONTENTS}

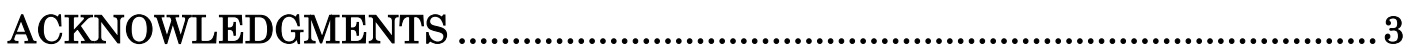

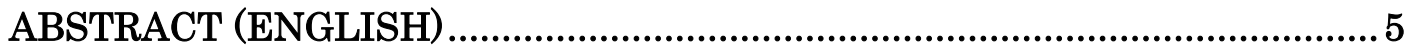

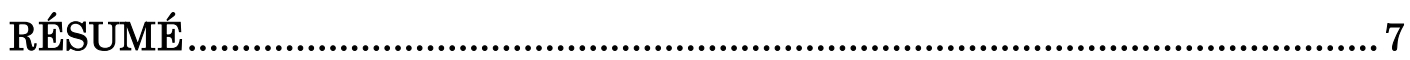

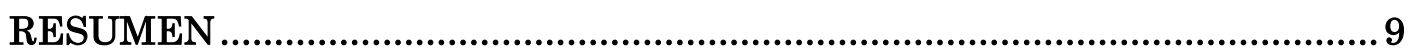

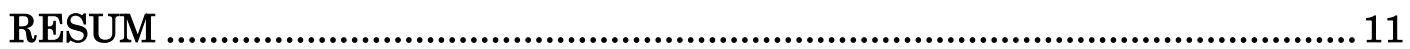

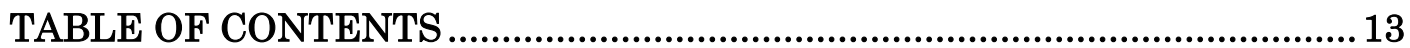

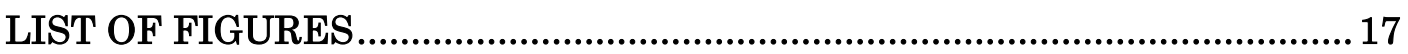

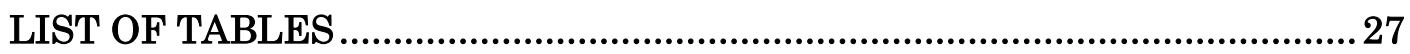

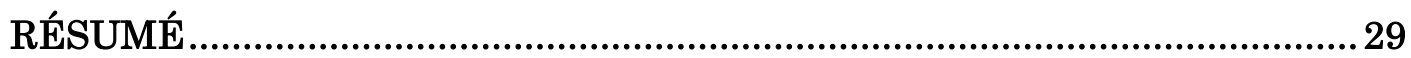

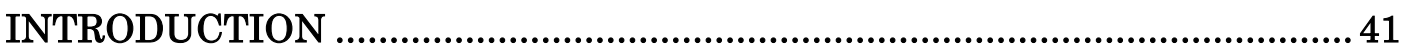

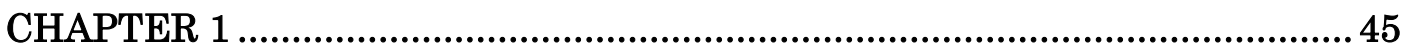

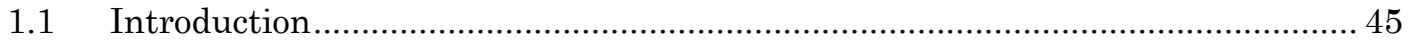


1.2 Microfluidic applications: microwave fluid sensing ........................................ 46

1.3 Increasing frequency for liquid characterization ....................................... 46

1.4 Measurement methods ............................................................................ 48

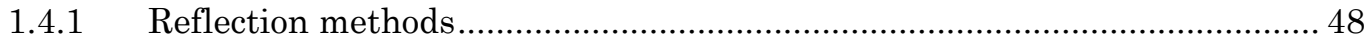

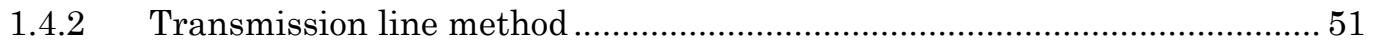

1.4.3 Resonant transmission lines ......................................................... 56

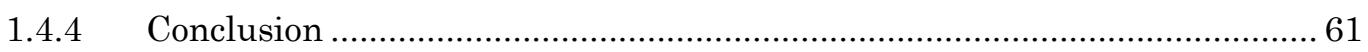

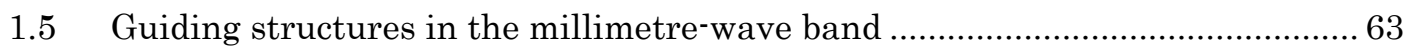

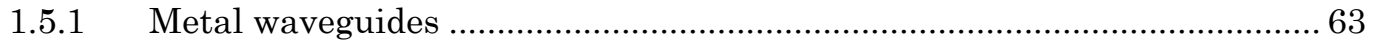

1.5.2 Printed planar transmission lines ...................................................... 63

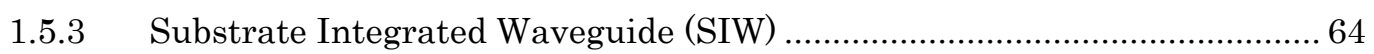

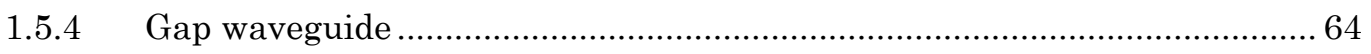

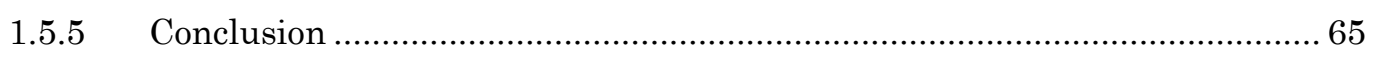

1.6 Manufacturing techniques for microfluidic sensors ..................................... 66

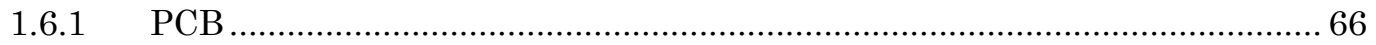

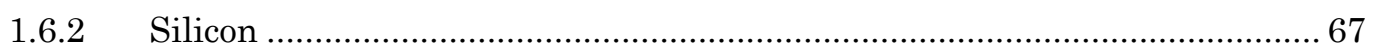

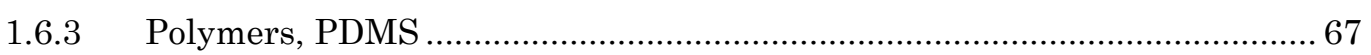

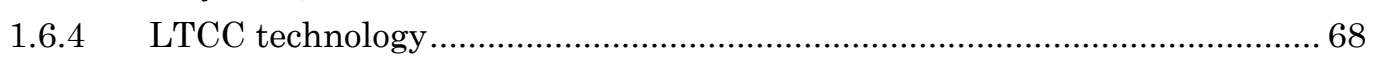

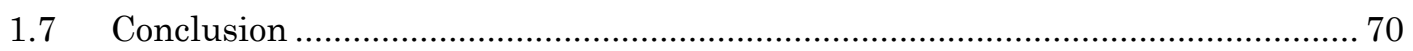

CHAPTER 2 ................................................................................ 71

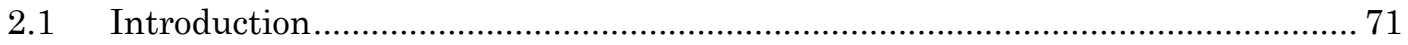

2.2 Overview of the gap waveguide technology ................................................... 72

2.2.1 Basic theory of the gap waveguide.......................................................... 72

2.2.2 Classification of the structures based on the gap waveguide concept......... 74

2.2.3 Principle of operation of the gap waveguide .......................................... 75

2.3 Design of the microstrip gap waveguide compatible with PCB or LTCC

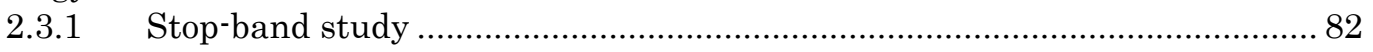

2.3.2 Impedance analysis methods .......................................................... 85

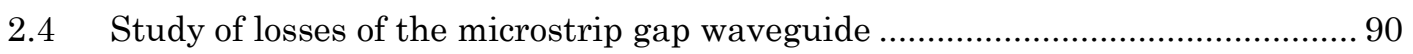

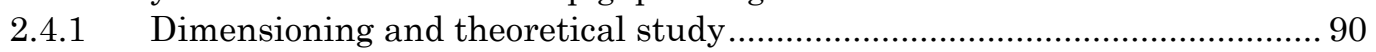

2.4.2 Influence of the gap height in the $\mathrm{Q}$-factor value: ....................................... 95

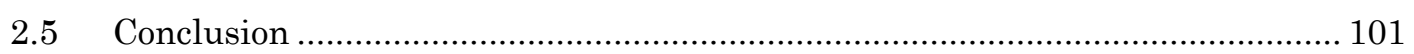

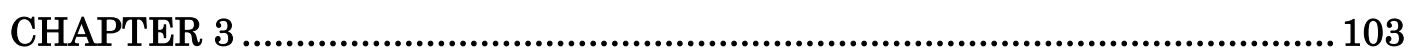

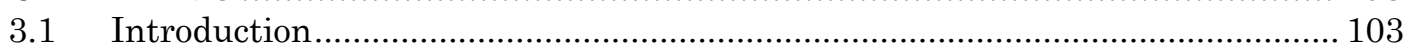

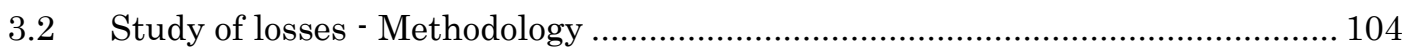

3.3 SIW and gap waveguide design .............................................................. 106

3.3.1 Design of the SIW structure ............................................................ 106

3.3.2 Design of the gap waveguide structure .............................................. 109

3.3.3 Computed results for both technologies ............................................... 110

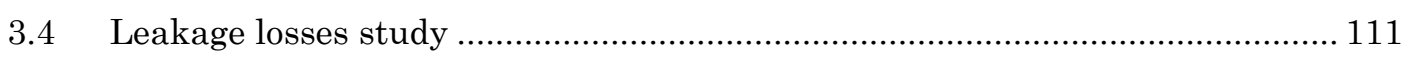

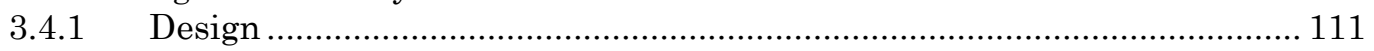




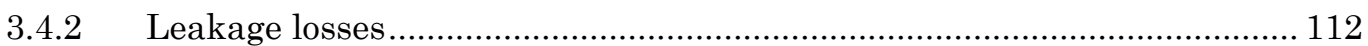

3.4.3 Comparison of the E-field distribution in gap waveguide and SIW .......... 117

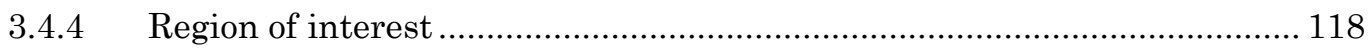

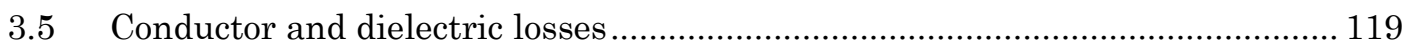

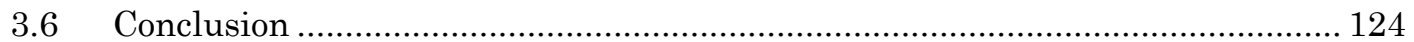

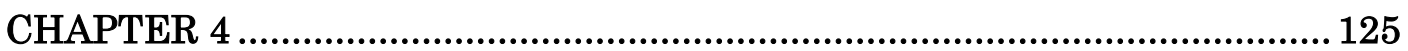

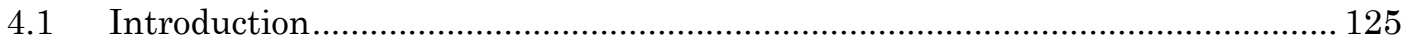

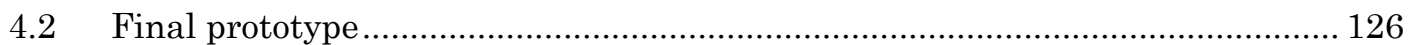

4.3 PCB and LTCC manufacturing techniques ............................................... 127

4.4 Gap waveguide design using PCB multilayer technique ................................ 129

4.4.1 Manufactured prototype with PCB.................................................... 135

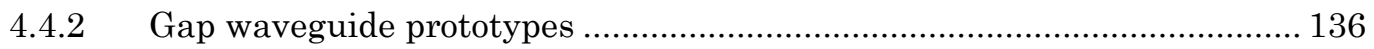

4.4.3 Transition CPW-to-microstrip-to-gap waveguide ....................................... 136

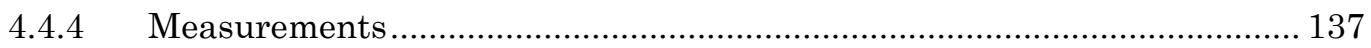

4.5 Gap waveguide design using LTCC technology …............................................. 140

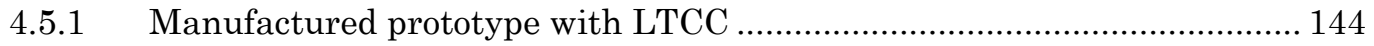

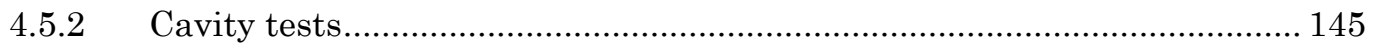

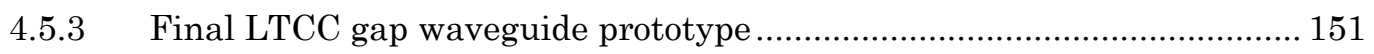

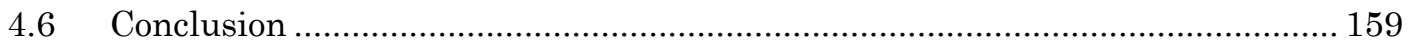

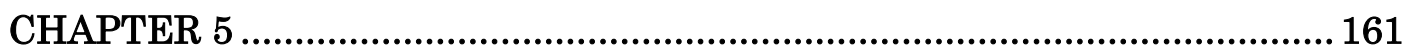

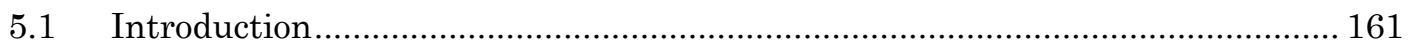

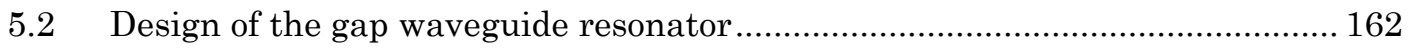

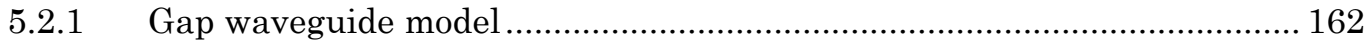

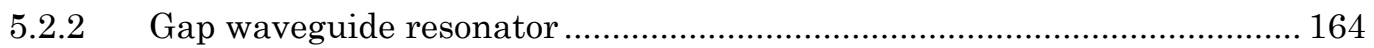

5.3 Comparison between gap waveguide and SIC for fluid detection ..................... 167

5.4 Manufacturing and measurements of the gap waveguide resonator with PCB

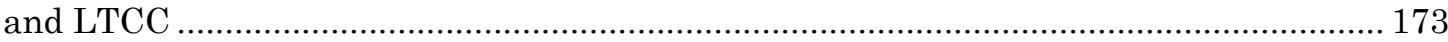

5.4.1 Gap waveguide with Printed Circuit Board (PCB) .................................... 173

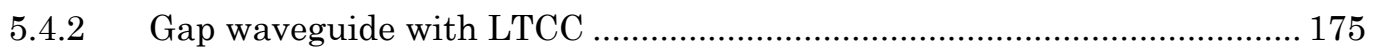

5.5 Other resonant structures based on gap waveguide ….................................. 180

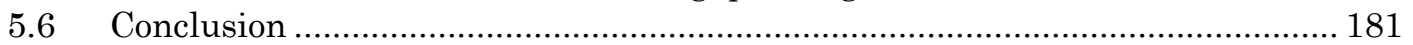

FINAL CONCLUSIONS AND FUTURE LINES ...................................... 183

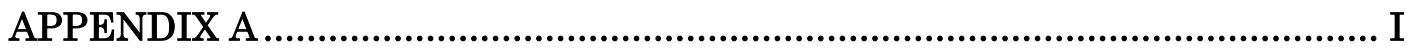

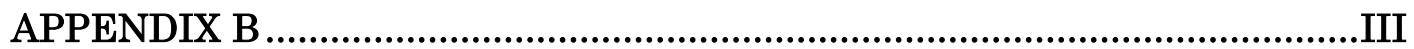

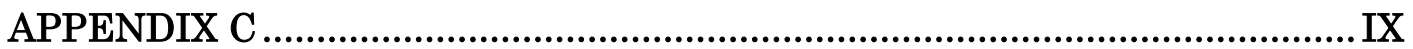

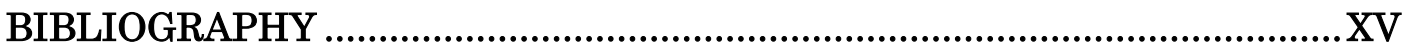




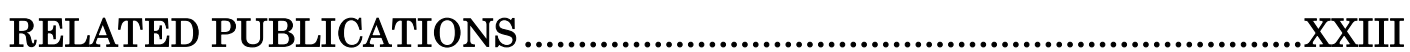




\section{List of Figures}

Figure 0.1. Résultats présentées dans [3] 31

Figure 0.2. Ridge gap waveguide: a) Géométrie; b) Vue de face.

Figure 0.3. Diagramme de dispersion de la " gap waveguide».

Figure 0.4. Distribution du champ électrique dans la surface de la plaque métallique supérieure.

Figure 0.5. Schéma complète du prototype: Transition coplanaire-microruban avec couplage par fente en $\mathrm{H}$, couche pour créer la cavité d'air et gap waveguide.

Figure 0.6. Comparaison entre les résultats en théoriques et expérimentaux de la " gap waveguide» fabriqué avec RO4003. 35

Figure 0.7. Section transversal du prototype "gap waveguide" avec LTCC. .... 36

Figure 0.8. Vue 3D du résonateur "gap waveguide" pour la detection des fluides.

Figure 0.9. Résultats théoriques présentées dans [6] de la structure SIC (a) et avec le résonatuer " gap waveguide " (b) avec la cavité vide, et remplie de méthanol et de l'eau. 38

Figure 0.10. Résultats théoriques et expérimentaux du résonateur "gap waveguide" avec RO4003. 38 
Figure 1.1. Measured a) dielectric constant b) conductivity from $500 \mathrm{MHz}$ to 20 $\mathrm{GHz}$ for various glucose levels presented in [4] ............................................ 47

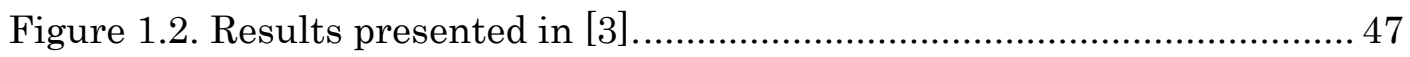

Figure 1.3. Illustration of the principle of the reflection method..................... 48

Figure 1.4. Principle of operation of the open ended coaxial method. .............. 49

Figure 1.5. Open-ended coaxial line reflection method presented in [5] ......... 50

Figure 1.6. Schematic and physical implementation of the sensor proposed in [8] 50

Figure 1.7. Measurement system presented in [8]. ....................................... 51

Figure 1.8. Transmission line method using coaxial line presented in [9]...... 52

Figure 1.9. Transmission method for material characterization using

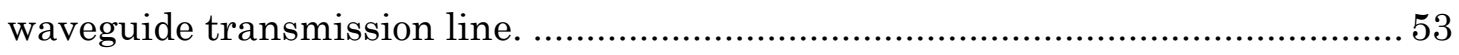

Figure 1.10. Measurement system presented in [12] ................................... 53

Figure 1.11. Measurement system based on microfluidic channels embedded in coplanar transmission lines proposed in [14]. ................................................. 54

Figure 1.12. Planar transmission line sensor illustrated in [15] ..................... 55

Figure 1.13. Proposed coplanar sensor presented in [3] ................................ 56

Figure 1.14. Cylindrical Cavity resonator at the TM0n0 mode. ...................... 57

Figure 1.15. Cylindrical resonator for sample characterization presented in [16]...... .57

Figure 1.16. Prototype of the dielectric disk resonator proposed in [17]......... 58

Figure 1.17. Dielectric sensor presented in [18]. .......................................... 58

Figure 1.18. Non-invasive glucose sensor proposed in [1] ............................59

Figure 1.19. Coplanar resonator presented in [20]....................................... 59

Figure 1.20. S-parameters for 5 ethanol/DI water mixtures at several volume

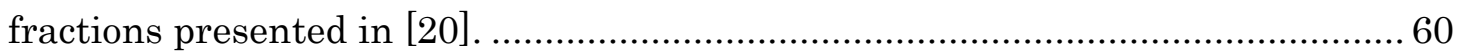

Figure 1.21. Transmission line sensor proposed in [21].................................6 60

Figure 1.22. Microstrip stub resonator presented in [22] ............................... 61

Figure 1.23. Cross section of the microstrip substrate (designed and

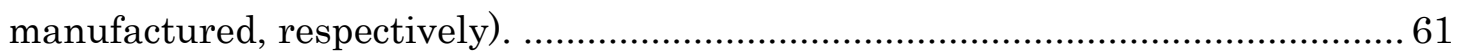

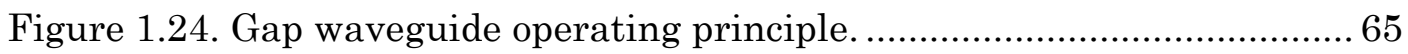

Figure 1.25. Microfluidic sensor based on PCB technology presented in [29].. 67

Figure 2.1 Linearly polarized hard waveguide.......................................... 73

Figure 2.2 No propagation between PEC and PMC condition when $h<\lambda / 4 \ldots \ldots .73$

Figure 2.3. Ideal gap waveguide. ................................................................. 73 


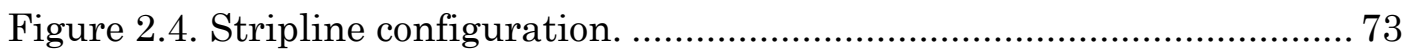

Figure 2.5. Geometry of the ridge gap waveguide. ........................................ 74

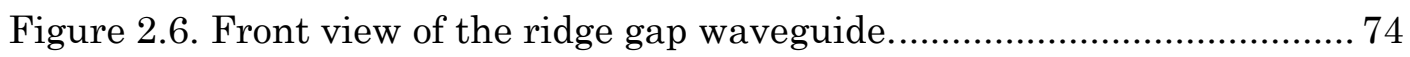

Figure 2.7. Ridge gap waveguide configuration............................................ 74

Figure 2.8. Groove gap waveguide - Vertical polarization. .............................. 75

Figure 2.9. HIS is made with a surface of pins........................................... 75

Figure 2.10. HIS made with mushroom shape surface embedded in dielectric. 75

Figure 2.11. Mushroom surface and strip share the same substrate.............. 75

Figure 2.12. Cross-section of a periodic pattern of metallic mushrooms.......... 76

Figure 2.13. Capacitance and inductance of the circuit model. ....................... 76

Figure 2.14. Periodic mushroom shape pattern............................................. 77

Figure 2.15. Brillouin zone of periodic pattern. .......................................... 77

Figure 2.16. Dispersion diagram of the mushroom surface. ........................... 77

Figure 2.17. Boundary definition to calculate dispersion diagram of periodic pins: a) Pin unit cell, b) Master boundary condition, c) Slave boundary condition.

Figure 2.18. Dispersion diagram created by the pin surface. ........................ 78

Figure 2.19. Dispersion diagram created by the mushroom surface. ............... 79

Figure 2.20. a) $3-\mathrm{D}$ view of the gap waveguide structure. b) Example of the boundary conditions in one row of pins to obtain the dispersion diagram............ 80

Figure 2.21. Dispersion diagram of one row of mushrooms and ridge. ........... 80

Figure 2.22. S-parameters of the gap waveguide with mushroom surface. ..... 80

Figure 2.23. Minimum and maximum frequency of the stop-band by varying

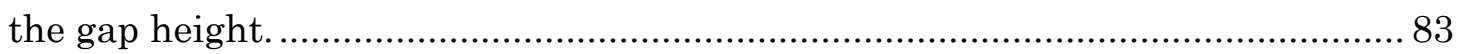

Figure 2.24. Gap height impact over the bandwidth of the stop-band............. 83

Figure 2.25. Impact of the ratio pin_periodicity/diameter over the minimum and maximum frequency of the stop-band with the mushroom surface. 84

Figure 2.26. Impact of the ratio pin_periodicity/diameter over the BW of the

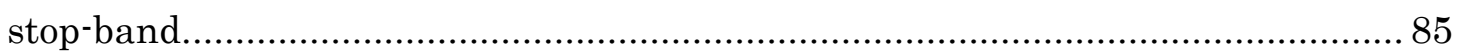

Figure 2.27. Front view of: a) Microstrip line, b) Gap waveguide.................... 85

Figure 2.28. Integration lines used in the simulations .................................. 87

Figure 2.29. Surface used in simulations to obtain the Poynting vector.......... 88

Figure 2.30. Five cross-sections to evaluate the characteristic impedance value.

Figure 2.31. Computed impedance value with the 3 methods for each crosssection. 89 
Figure 2.32. Gap waveguide short-circuit resonator using Eigenmode solver of HFSS.

Figure 2.33. Top view of the short-circuit gap waveguide resonator............... 93

Figure 2.34. Excited third-order resonant mode.............................................. 93

Figure 2.35. Three configurations based on gap waveguide concept............... 94

Figure 2.36. Dispersion diagram of the configuration with strip and pins below

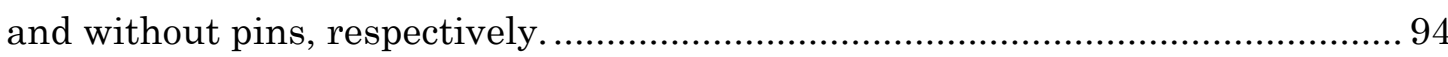

Figure 2.37. Computed $\mathrm{Q}$-factor versus gap height at $\mathrm{f}=60 \mathrm{GHz} \ldots \ldots \ldots \ldots \ldots \ldots . . . . . . . .96$

Figure 2.38. Computed minimum and maximum frequency of the stop-band. 96

Figure 2.39. Computed $\mathrm{Q}$-factor versus gap height for the three configurations considering dielectric and conductor losses.

Figure 2.40. Computed Q-factor due to the conductor loss in function of the gap height.

Figure 2.41. Front view of the E-field propagated in the three configurations for the third order mode of resonance for hgap $=417 \mu \mathrm{m}$ (considering no losses).. 98

Figure 2.42. Q-factor due to conductor, dielectric or both losses for the ridge and transmission line configurations.

Figure 2.43. Attenuation constant due to conductor, dielectric or both losses for the ridge and transmission line configurations.

Figure 2.44. Q-factor as a function of the width of the strip. 100

Figure 3.1. Flowchart diagram of the losses study procedure. 106

Figure 3.2. SIW technology. a) Three-dimensional view of a SIW structure; b) Front view of SIW.

Figure 3.3. Relationship between operating frequency $(\mathrm{GHz})$ and the minimum distance between vias $\mathrm{p}(\mathrm{mm})$ and the minimum via diameter in SIW structures.

Figure 3.4. Front view of Gap Waveguide with LTCC. 109

Figure 3.5. Computed dispersion diagram for SIW and gap waveguide, respectively. 110

Figure 3.6. Computed S11 and S21 of the SIW with LTCC........................ 111

Figure 3.7. Computed S11 and S21 of the LTCC gap waveguide................. 111

Figure 3.8. Computed leakage attenuation versus $\mathrm{p} / \mathrm{d}$. 114

Figure 3.9. Impact of the variation of $\mathrm{p}$ over the stop-band for the gap waveguide.

Figure 3.10. E-field distribution in the dielectric for SIW and in the air gap for GW for the relationship $\mathrm{p} / \mathrm{d}=4.1$ (considering no lossy materials). 116 
Figure 3.11. Evolution of the leakage losses (dB/m) varying the distance x. . 117

Figure 3.12. Vertical E-field distribution at the surface of the upper metal plate

Figure 3.13. Region of interest for the SIW and gap waveguide in the plane of $\mathrm{d} / \mathrm{\lambda c}, \mathrm{p} / \mathrm{\lambda c}$.

Figure 3.14. Design and computed Q-factor for both configurations. 120

Figure 3.15. Properties of the materials used in Simulations: Ideal conductor and lossy dielectric.

Figure 3.16. Properties of the materials used in simulations: Ideal dielectric and lossy conductors 121

Figure 3.17. Properties of the materials: Ideal dielectric, ideal vias metallization (PEC) and losses in the upper and lower metal plate. 122

Figure 3.18. Case 1: with lossy vias and plates; Case 2: With conductor loss in plates and vias without loss.

Figure 3.19. Q-factor obtained with the Eigenmode of HFSS considering dielectric, conductor, both or any losses. 123

Figure 4.1. LTCC module example. 69

Figure 4.2 Breadboard of the complete structure: Coplanar to microstrip to $\mathrm{H}^{-}$ shape coupled aperture transition, cavity layer to create the air gap and gap waveguide including the surface of vias embedded in a substrate and transmission line. 127

Figure 4.3. Difficulties in the via filling after co-firing. 128

Figure 4.4. Via filling difficulties: Pictures 1 and 2 from left to right: Shift in the via filling due to the misalignment in the thick film screen printer. Picture 3 from left to right: insufficient paste quantity applied to the vias 128

Figure 4.5. Misalignment due to lamination process. ................................. 129

Figure 4.6. Dispersion diagram of the gap waveguide with RO4003. ............ 130

Figure 4.7. Gap waveguide with R04003 substrate. a) Transmission and reflection coefficients b) Wave port excitation in HFSS. 131

Figure 4.8. Front view and dimensions of the aperture coupled microstrip to gap waveguide transition. 131

Figure 4.9. Side view of the microstrip to gap waveguide transition. ............ 132

Figure 4.10. H-shape aperture microstrip to gap waveguide transition. ....... 132

Figure 4.11. Microstrip-to-gap waveguide back-to-back transition: a) 3D view of the structure with HFSS, b) E-field distribution in the wave port excitation.132 
Figure 4.12. Computed results for the transmission and reflection coefficients of the microstrip to gap waveguide transition. 133

Figure 4.13. Efficiency of the aperture in terms of radiation loss. ................ 133

Figure 4.14. Dimensions of the coplanar probe............................................. 134

Figure 4.15. Computed results of the back-to-back CPW-to-Microstrip-to Gap Waveguide transition: a) E-field distribution at the Wave Port, b) Computed Sparameters $(\mathrm{dB})$ 134

Figure 4.16. a) Picture with the addition of the RO4003 layer to create the air gap cavity; b) Three parts (gap waveguide, transition and layer for the air cavity) before stacking. 135

Figure 4.17. Final prototype including the transition and the gap waveguide. 136

Figure 4.18. a) Layout of the six prototypes with ADS software; b) Manufactured device with RO4003 substrate. ................................................. 136

Figure 4.19. Manufactured device with RO4003 including the SIW transitions and a calibration kit at right. 137

Figure 4.20. CPW radial stub-microstrip transition.................................... 137

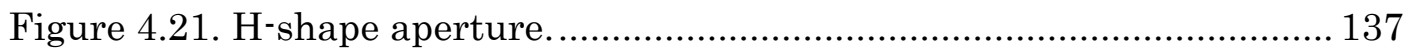

Figure 4.22. RF probe station measurement and GSG (ground signal ground) probe with the coplanar to microstrip transition 138

Figure 4.23. Comparison between computed results and measurements for the gap waveguide with RO4003. 138

Figure 4.24. Transmission and reflection coefficient for air height (hgap) $0.3 \mathrm{~mm}$ and $0.2 \mathrm{~mm}$ and front view of the gap waveguide, respectively. 139

Figure 4.25. Top view of the gap waveguide with two $90^{\circ}$ bends. E-field view in the ground plane with the $\mathrm{H}$-shape apertures at $52 \mathrm{GHz}$. 139

Figure 4.26. Computed results and measurements of the gap waveguide with two 90 bends. 140

Figure 4.27. Geometry and front view of the gap waveguide with LTCC. ..... 141

Figure 4.28. Dispersion diagram created by the gap waveguide with LTCC. 141

Figure 4.29. Transmission and reflection coefficient for the gap waveguide designed with ESL41110, with and without conductor and dielectric losses. .... 142

Figure 4.30. Computed results of the complete LTCC structure, including gap

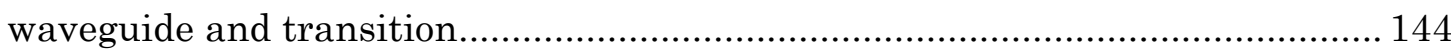

Figure 4.31. Flowchart of cavity fabrication process................................. 145

Figure 4.32. Cross-section of the LTCC open cavity..................................... 146

Figure 4.33. Six open cavities included in the LTCC prototype..................... 146 
Figure 4.34. Relationship between obtained and designed dimensions for each open cavity 147

Figure 4.35. Geometry of the internal cavities. 148

Figure 4.36. Manufactured prototype with two internal cavities: one with holes for the SVM evaporation and other without them. ........................................... 148

Figure 4.37. Laser cut of the first cavity.................................................... 149

Figure 4.38. Picture of the internal cavities: a) Resin base to analyze performances inside the cavity; b) First part of the cavity; c) At the center of the cavity. 149

Figure 4.39. Pictures of the second test of LTCC cavities: side and top view. 150

Figure 4.40. Second test for the LTCC manufacturing of internal cavities. a) Manufactured prototype with the three cavities; b) Backlit image of the prototype. 150

Figure 4.41. Designed and obtained dimensions of the internal cavities

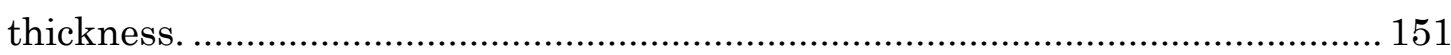

Figure 4.42. Cross-section of the LTCC gap waveguide prototype. ................ 152

Figure 4.43. Laser cutting: holes and cavities. ........................................... 152

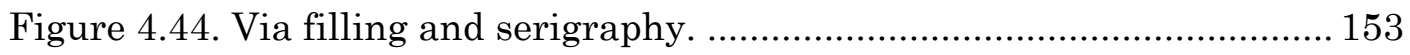

Figure 4.45. Stacking of all the layers........................................................ 153

Figure 4.46. Ablation process to create the gap for the CPW transition: before

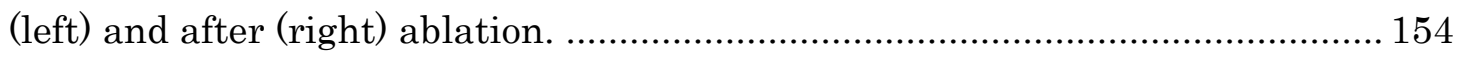

Figure 4.47. Final manufactured prototype with LTCC.............................. 154

Figure 4.48. Measurements with the GSG probe of the LTCC prototype. .... 155

Figure 4.49. Comparison between measurements and computed results of the

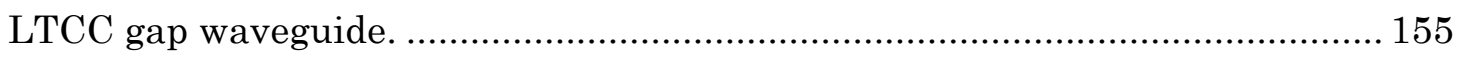

Figure 4.50. Resin base created for measuring the dimensions in the cut plane

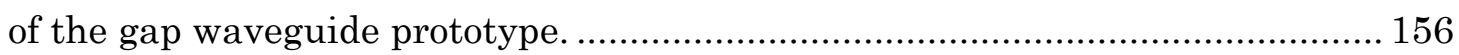

Figure 4.51. Cut of the gap waveguide prototype with LTCC. Dimensions are specified and the problems obtained related to the serigraphy of the gold and the

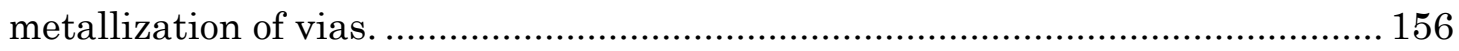

Figure 4.52. Overflow of the gold of the ground plane into the cavity........... 157

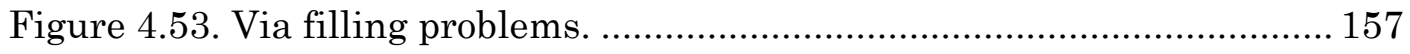

Figure 4.54. Overflow of the gold metallization. Gold thickness $\approx 50 \mu \mathrm{m} . \ldots \ldots . .158$

Figure 4.55. Shift of the aperture related to the central via......................... 158

Figure 4.56. Insertion of a "metallic patch" in each LTCC layer to solve the via filling problems. 158 
Figure 5.1. Gap waveguide geometry with LTCC 162

Figure 5.2. Computed dispersion diagrams: stop-band created by the mushroom surface (HIS) and dispersion diagram of all the structure with the quasi-TEM mode propagated, respectively. 163

Figure 5.3. S-parameters of the gap waveguide topology.............................. 164

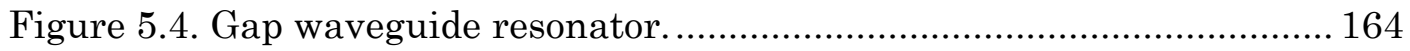

Figure 5.5. Front view of the gap waveguide resonator: Without and with the LTCC wall to separate the air cavity from the microfluidic channel. ................. 165

Figure 5.6. Material properties and boundary condition in the gap zone between the pins and the upper metal plate. 165

Figure 5.7. Gap waveguide resonator with LTCC wall separating the air cavity from the microfluidic channel. 166

Figure 5.8. S-parameters of the gap waveguide resonator including the LTCC wall to separate the air cavity from the microfluidic channel. 166

Figure 5.9. E-field distribution in the air gap cavity, LTCC wall and air channel. 167

Figure 5.10. Cavity resonator based on Substrate Integrated Cavity at $60 \mathrm{GHz}$ [80] 168

Figure 5.11. 3D view of the gap waveguide stub resonator for fluid detection. 168

Figure 5.12. Front view of the gap waveguide resonator. 168

Figure 5.13. Computed results of the SIC sensor presented in [6] of the cavity loaded with air, methanol and water. 169

Figure 5.14. Transmission and reflection coefficient for fluidic cavity filled with: air, $\varepsilon r=7$ and $\varepsilon r=13$. 170

Figure 5.15. Transmission coefficient for the fluidic cavity with: air, $\varepsilon r=7$ and $\varepsilon r=13$. 170

Figure 5.16. Transmission coefficient for the fluidic channel: empty, filled with methanol and with water. 172

Figure 5.17. Resonant frequency in function of the permittivity for the 3 cases: empty channel, with methanol and water. 172

Figure 5.18. Six gap waveguide prototypes using RO4003 as substrate. 173

Figure 5.19. Gap waveguide resonator, ground plane with $\mathrm{H}$-shape apertures and coplanar to microstrip transition manufactured with RO4003 174

Figure 5.20. Computed and measured results for the gap waveguide resonator GW4 174 
Figure 5.21. Computed and measured results for the gap waveguide resonator GW6...... 175

Figure 5.22. Manufactured LTCC prototype................................................. 176

Figure 5.23. Pictures of the stacking process of the LTCC prototype............. 176

Figure 5.24. Top view of the 3X/4 resonator using LTCC and cavity dimensions. 177

Figure 5.25. Comparison between computed and measured results for the $3 \mathrm{~N} / 4$ gap waveguide resonator using LTCC. 178

Figure 5.26. Top view of the 5X/4 resonator and cavity dimensions.............. 178

Figure 5.27. Dimensions of the LTCC wall designed and measured.............. 179

Figure 5.28. Computed and measured results for the 5N/4 gap waveguide resonator. 179

Figure 5.29. Manufactured LTCC prototype. Bad gold serigraphy in the transition 3 for the $5 \lambda / 4$ gap waveguide resonator. Hard to make contact with the probes used for measurements. 180

Figure 5.30. Proposed ring resonator based on gap waveguide technology and electromagnetic response. 181

Figure 5.31. Gap waveguide ring resonator with the microfluidic cavity. ..... 181

Figure 1. Manufacturing steps in LTCC. ...................................................... II

Figure 2.Schema for the layout processing for LTCC................................... IV

Figure 3. Laser machine ASTREE 250 model...............................................

Figure 4. Example of drilled holes with laser machining................................

Figure 5. Stacking LTCC layers ................................................................. VI

Figure 6. LTCC layer under vacuum to eliminate air between layers ............. VI

Figure 7. Uniaxial press machine for lamination process .............................VII

Figure 8. Temperature profile for LTCC process ............................................VII

Figure 9. Coplanar-to-microstrip transition (top view) ....................................

Figure 10. Manufactured CPW-to-microstrip transition with RO4003............ XI

Figure 11. Layout of the ground plane with the $\mathrm{H}$-shape apertures (bottom

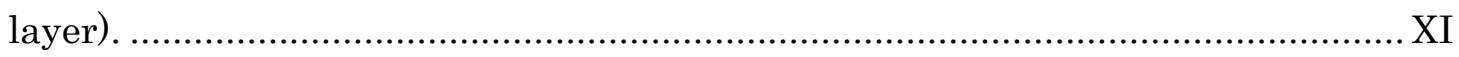




\section{List of Tables}

Table 1.1. State of the art of the measurement methods of fluids at the microwave range. 62

Table 1.2. Properties of each guiding structures according to our objectives... 66

Table 1.3. Manufacturing techniques properties according to our objectives. . 69

Table 2.1. Vertical E-field distribution at the surface of the upper metal plate.

Table 2.2. Gap waveguide parameters. 81

Table 2.3. Values of the characteristic impedance calculated by means of the four methods at the center of the structure. 88

Table 2.4. Result of the three methods in 3 cross-section positions.................. 89

Table 2.5. Characteristics of the two LTCC tapes used in this study............... 92

Table 2.6. Comparison of $\mathrm{Q}$-factor as a function of mushroom-shape and pin surface. 93

Table 2.7. Q-factor and attenuation constant for each air gap height.............. 95

Table 2.8. Computed results of $\mathrm{Q}$ and $\mathrm{a}$ for the three configurations considering: lossy materials, only loss in the dielectric and only loss in the conductor. 98

Table 2.9. Q-factor versus the strip width.................................................. 100 
Table 3.1. Common dimensions of the SIW and gap waveguide design. 109

Table 3.2 Common parameters for both resonators (SIW and gap waveguide).

Table 3.3. Specific design parameters for SIW and gap waveguide.

Table 3.4. S-parameters and leakage attenuation computed with the Driven modal at $170 \mathrm{GHz}$ (considering no losses)

Table 3.5. Impact of p/d over the stop-band of gap waveguide and the fc of SIW (distance $\mathrm{x}=$ wridge $+1.5 \mathrm{sp}$, hgap $=200 \mathrm{um}$ )

Table 3.6. Computed $\mathrm{Q}$-factor due to the dielectric loss (no conductor losses are considered)

Table 3.7. Computed Q-factor due to the conductor loss (no dielectric losses are considered)

Table 3.8. Conductor loss characterization: Gold for upper and lower metal plate, PEC for vias. 122

Table 4.1. Dimensions of the gap waveguide design using RO4003 substrate.

Table 4.2. Dimensions of the gap waveguide design using LTCC substrate.. 141

Table 4.3. Transmission losses with and without losses for two frequencies as reference

Table 4.4. Description of the model: Gap waveguide with LTCC. 143

Table 4.5. E-field propagated in the air gap for three specific frequencies: 45 $\mathrm{GHz}$ (outside the stop-band), $52 \mathrm{GHz}$ (in the middle of the stop-band) and $62 \mathrm{GHz}$ (outside the stop-band)

Table 4.6. Pictures of the open cavities before and after co-firing.

Table 5.1. Design parameters of the gap waveguide model with LTCC 163

Table 5.2. Computer resonant frequency and comparison between SIC and gap waveguide.

Table 5.3. Dielectric properties of the fluids under test at $60 \mathrm{GHz}$. 171

Table 1. Dimensions of the RO4003 gap waveguide prototype. $\mathrm{X}$ 


\section{RÉSUMÉ}

\section{Introduction générale}

Les systèmes micro-fluidiques ont émergé comme une technologie prometteuse pour l'analyse moléculaire, la bio-défense et la micro-électronique. Les propriétés des systèmes micro-fluidiques, tels que le traitement des échantillons et le contrôle de précision des fluides, en ont fait des candidats intéressants pour remplacer les approches expérimentales traditionnelles. Les dispositifs microfluidiques se caractérisent par des canaux fluidiques avec des dimensions de l'ordre de quelques dizaines à quelques centaines de micromètres.

L'analyse de fluides par des capteurs micro-ondes offre de nouvelles possibilités pour la caractérisation des milieux par des méthodes non invasives. L'importance de la mesure diélectrique des fluides est liée à l'extraction des caractéristiques électriques ou magnétiques des matériaux, qui se sont avérés utiles dans de nombreux domaines de recherche et de développement, tels que la biologie moléculaire et le diagnostic médical.

Plusieurs techniques sont disponibles dans le domaine fréquentiel pour analyser les propriétés diélectriques des liquides et leur composition. Nous nous concentrons sur les techniques de cavité résonante pour la caractérisation des fluides dans la gamme des ondes millimétriques. Cependant, ces techniques ne sont pas compatibles avec le processus de laboratoire "lab-on-chip " en raison de ses dimensions. Dans ce contexte, une nouvelle structure appelée "gap 
waveguide" apparaît comme un bon candidat pour surmonter les principaux inconvénients des cavités résonnantes classiques.

Cette thèse présente le développement de la technologie "gap waveguide " dans la bande d'ondes millimétriques en termes de conception et de pertes. D'autres technologies classiques sont discutées ainsi, afin de comparer avec leurs performances en termes de pertes avec celles de la " gap waveguide».

Nous présentons également la conception d'un résonateur basé sur la « gap waveguide" capable de travailler pour la détection micro-fluidique. Dans ce contexte, nous proposons une étude comparative entre la "gap waveguide " et la technologie "Substrate Integrated Cavity" (SIC) en termes de caractérisation des fluides à $60 \mathrm{GHz}$. Dans ce but, plusieurs prototypes ont été fabriqués avec le substrat RO4003 et la technologie "Low Temperature Co-fired Ceramic " (LTCC). Un important travail dans le laboratoire LTCC a été fait dans le but de valider les étapes essentielles du processus LTCC pour la fabrication de la "gap waveguide", en particulier celles liées à la réalisation de cavités (externes et internes).

\section{Chapitre 1 : Motivation et état de l'art}

Ce chapitre présente la motivation et les objectifs de cette thèse. Dans ce but, d'abord, nous présentons les applications micro-fluidiques dans la gamme des ondes millimétriques. Ensuite, les méthodes de mesures principales pour la caractérisation des fluides dans la gamme des micro-ondes sont examinées. Les structures de guidage compatibles avec ces méthodes sont également présentées. Le choix de la structure de guidage est basée sur trois caractéristiques : compatibilité avec la bande d'ondes millimétriques, minimisation des pertes RF et capacité de créer des cavités et des canaux où le champ électromagnétique est maximal. La technologie "gap waveguide" a été identifiée comme une solution prometteuse pour surmonter ces trois exigences. Une fois le choix de la structure de guidage effectué, les techniques de fabrication ont été analysées pour les applications micro-fluidiques.

Notre objectif général est de définir une structure capable de caractériser les propriétés électriques des fluides dans la bande des ondes millimétriques. En effet l'intérêt d'augmenter la fréquence est motivé par l'amélioration de la sensibilité des caractéristiques du fluide aux fréquences millimétriques. La Figure 0.1) présente le contraste de concentration en éthanol (Figure 0.1-a) et en 
cellules lymphatiques (Figure 0.1-b)) [3]. La différence de concentration ou de quantités de cellules impact plus sur la conductance aux hautes fréquences $(>20$ $\mathrm{GHz}$ ) que pour les fréquences plus basses.

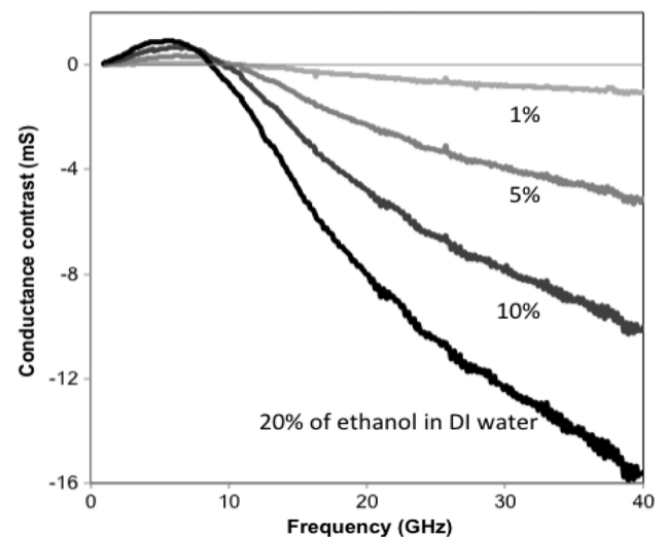

a) Conductance en fonction du contraste de concentration en éthanol

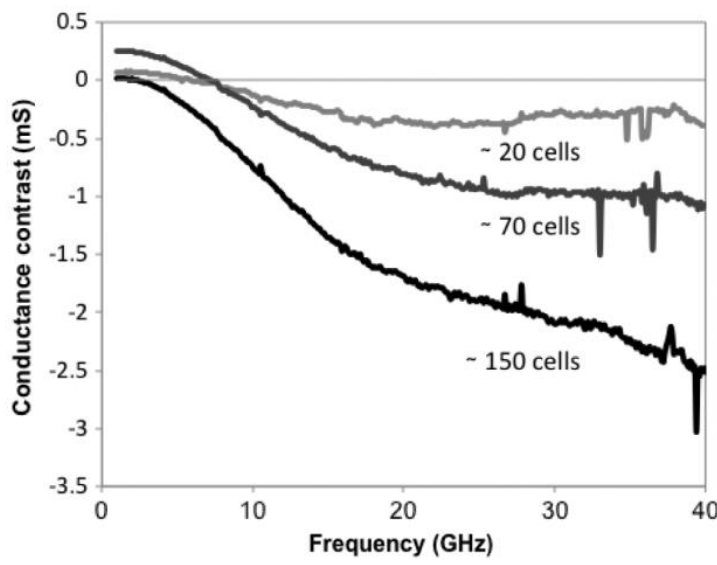

b) Conductance en fonction du pourcentage de cellules

Figure 0.1. Résultats présentées dans [3].

Ensuite, un état de l'art des méthodes de mesure pour la caractérisation des fluides à la gamme des micro-ondes a été fait. La littérature montre que, en termes de précision, la meilleure solution est basée sur des cavités résonnantes. Cependant, leur volume important empêche les applications de laboratoire sur puce.

Ensuite, nous nous sommes concentrés sur le choix de la structure capable de travailler dans la bande d'ondes millimétriques avec de faibles pertes et compatibles avec les méthodes de caractérisation du fluide de mesure. Dans ce contexte, une nouvelle technologie appelée " gap waveguide" présente de bonnes propriétés pour les deux exigences. Dans cette structure la propagation des ondes se fait dans la cavité d'air, qui permet de créer résonateurs basés sur cette technologie.

\section{Chapitre 2: Technologie "Gap Waveguide": Situation actuelle et contributions}

La technologie "gap waveguide" est présentée dans ce chapitre. Dans la bande d'ondes millimétriques, les contraintes techniques en termes de dimensions et de fabrication des structures de guidage classiques deviennent plus importantes. 
Dans ce contexte, ces dernières années, la technologie "gap waveguide " a émergé comme une nouvelle technologie de guidage qui peut surmonter les inconvénients de cette technologie. La structure classique de la "gap waveguide" est représentée dans la Figure 0.2. Cette structure se compose d'une ligne de transmission ou "ridge», une surface de pins métalliques et deux plaques métalliques. Quand la hauteur des pins est $\mathrm{\lambda} / 4$, cette surface crée une condition de haute impédance qui empêche la propagation dans toutes les directions, confinant ainsi le maximum du champ électrique dans la zone souhaitée (Figure $0.3)$.

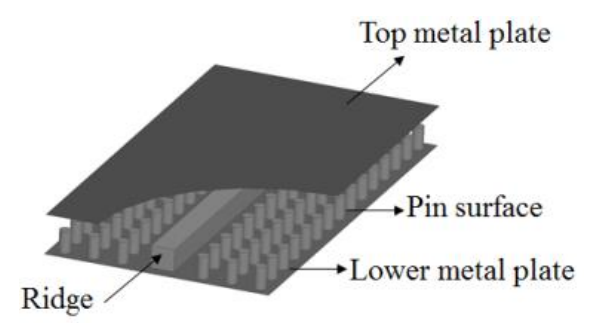

a)

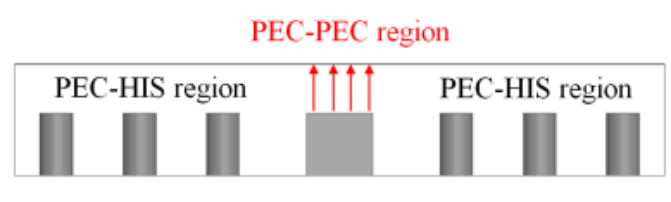

b)

Figure 0.2. Ridge gap waveguide: a) Géométrie; b) Vue de face.

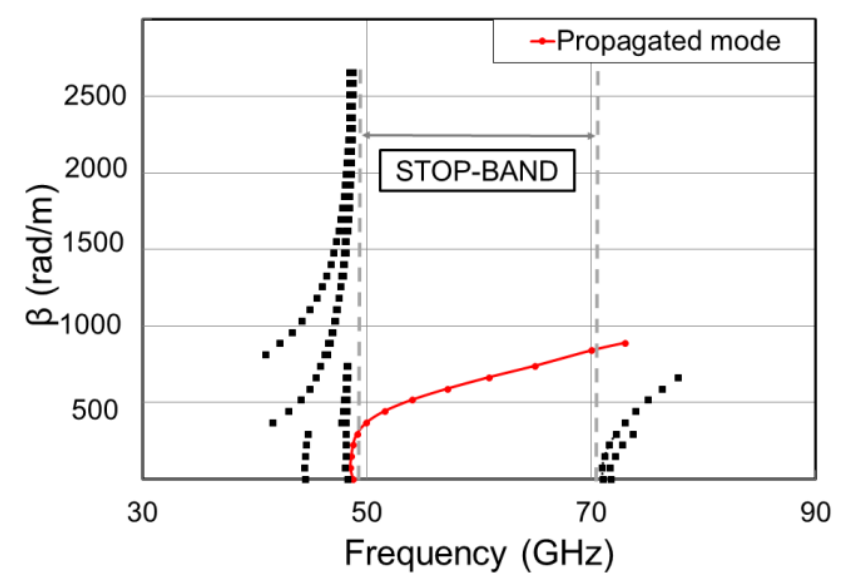

Figure 0.3. Diagramme de dispersion de la «gap waveguide».

Suite la présentation du fonctionnement de la "gap waveguide", une conception de cette technologie compatible avec la technologie PCB ou LTCC est montrée. Des études numériques relatives à la conception et le calcul de l'impédance caractéristique de ces structures ont été présentées. Les résultats 
permettent de caractériser ce type de structures et de fournir des valeurs de référence pour sa conception.

Enfin, on montre une évaluation des pertes par le facteur de qualité $Q$ de la " gap waveguide» incorporant diélectrique et compatible avec PCB ou LTCC. À la suite de cette caractérisation, pour la technologie " gap waveguide " de nombreux facteurs interviennent à la détermination du facteur $\mathrm{Q}$, comme la distance entre la ligne de transmission et la rangée adjacente de pins qui forment la surface HIS. Une analyse importante du facteur $\mathrm{Q}$ en fonction du rapport p/d (périodicité sur diamètre de pin) a été présentée. Cette caractérisation a été réalisée pour être en mesure de faire une comparaison postérieure avec d'autres technologies qui sont soumis à des normes de conception plus strictes en termes de ce rapport $\mathrm{p} / \mathrm{d}$, telle que la technologie SIW, comme on le verra dans le chapitre 3.

\section{Chapitre 3 : Comparaison entre les technologies « gap waveguide » et SIW}

Dans le troisième chapitre, une comparaison entre "gap waveguide " et SIW est présentée. Ces études ont été faites à $170 \mathrm{GHz}$ pour révéler les limites de fonctionnement des deux technologies en termes de pertes du conducteur, diélectrique et de fuite.

Dans un premier temps, la méthodologie employée pour l'étude des pertes est détaillée. Cette procédure consiste à analyser les paramètres de transmission pour la caractérisation des pertes de fuite. Dans un deuxième temps, les propriétés de dispersion de résonateurs sont étudiées afin d'obtenir le facteur $\mathrm{Q}$ qui permet de caractériser la constante d'atténuation.

Ensuite, la conception de la "gap waveguide" et la structure SIW fonctionnant à $170 \mathrm{GHz}$ est présentée. Les limites de conception en termes de par le diamètre et la périodicité entre les vias ont été appliquées pour le calcul des pertes de fuite. Les résultats montrent que la " gap waveguide " permet un range plus flexible pour la valeur de la periodicité et le diamètre de vias que la structure SIW où les pertes de fuites sont plus importantes selon la relation $\mathrm{p} / \mathrm{d}$ augmente (Figure 0.4).

Enfin, les pertes conductrices et diélectriques sont calculées par l'analyse du facteur Q des deux résonateurs. Deux conclusions méritent d'être mentionnées: 1) l'impact des pertes diélectriques est plus important dans la SIW que dans la « gap 
waveguide » car la propagation dans le premier cas se fait dans le diélectrique. 2) les pertes de conduction sont légèrement plus importantes dans la "gap waveguide " que dans SIW, dû à la surface de vias métalliques.

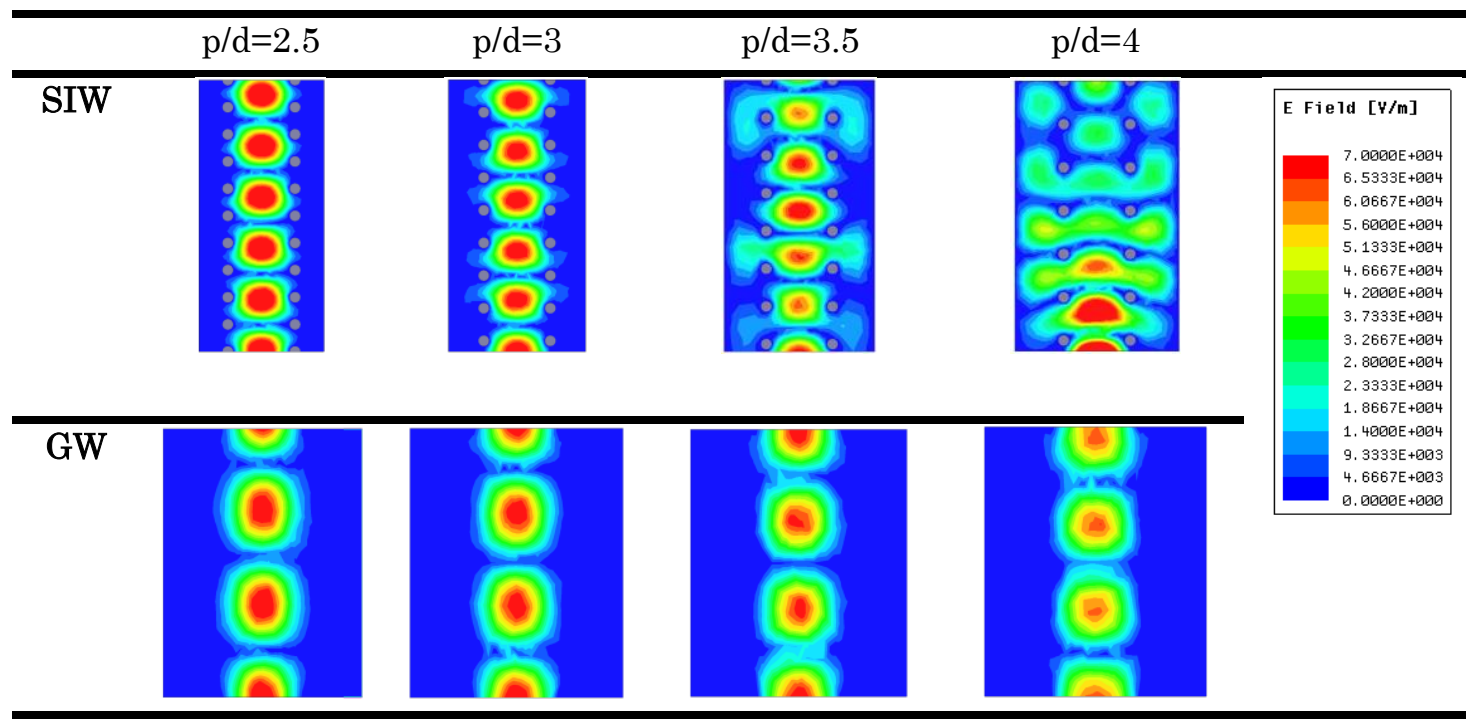

Figure 0.4. Distribution du champ électrique dans la surface de la plaque métallique supérieure.

En résumé, la technologie "gap waveguide" surmonte certaines limitations technologiques de SIW en termes des pertes de fuite et des pertes de diélectrique. En termes de pertes de conducteur, SIW présente un facteur Q plus élevé. Le choix de la structure dépend de l'application et les limitations de la technique de fabrication.

\section{Chapitre 4: Gap waveguide avec PCB et LTCC}

Le quatrième chapitre présente la mise en œuvre physique de la "gap waveguide" avec les technologies PCB et LTCC. Tout d'abord, la conception de la " gap waveguide» avec le substrat RO4003 a été expliquée. Ensuite, la transition pour l'alimentation de la "gap waveguide» est analysée. Cette transition se compose d'une ligne microruban couplée à la "gap waveguide " à partir d'une fente en forme $\mathrm{H}$. La forme de la fente réduit les fuites et la taille par rapport aux fentes rectangulaires. Ensuite, une ligne coplanaire est ajoutée dans une configuration « back-to-back» pour la mesure des prototypes (Figure 0.5).

Les résultats expérimentaux sont bons en comparaison avec les valeurs théoriques pour la " gap waveguide " en utilisant le substrat RO4003 (Figure 0.6). Néanmoins, la dépendance entre la hauteur du gap d'air et l'impédance 
caractéristique a été observée, étant une limitation technologique des circuits imprimés multicouches. Dans ce contexte, la technologie LTCC devient un bon candidat afin surmonter ces limitations en termes de contrôle des cavités.

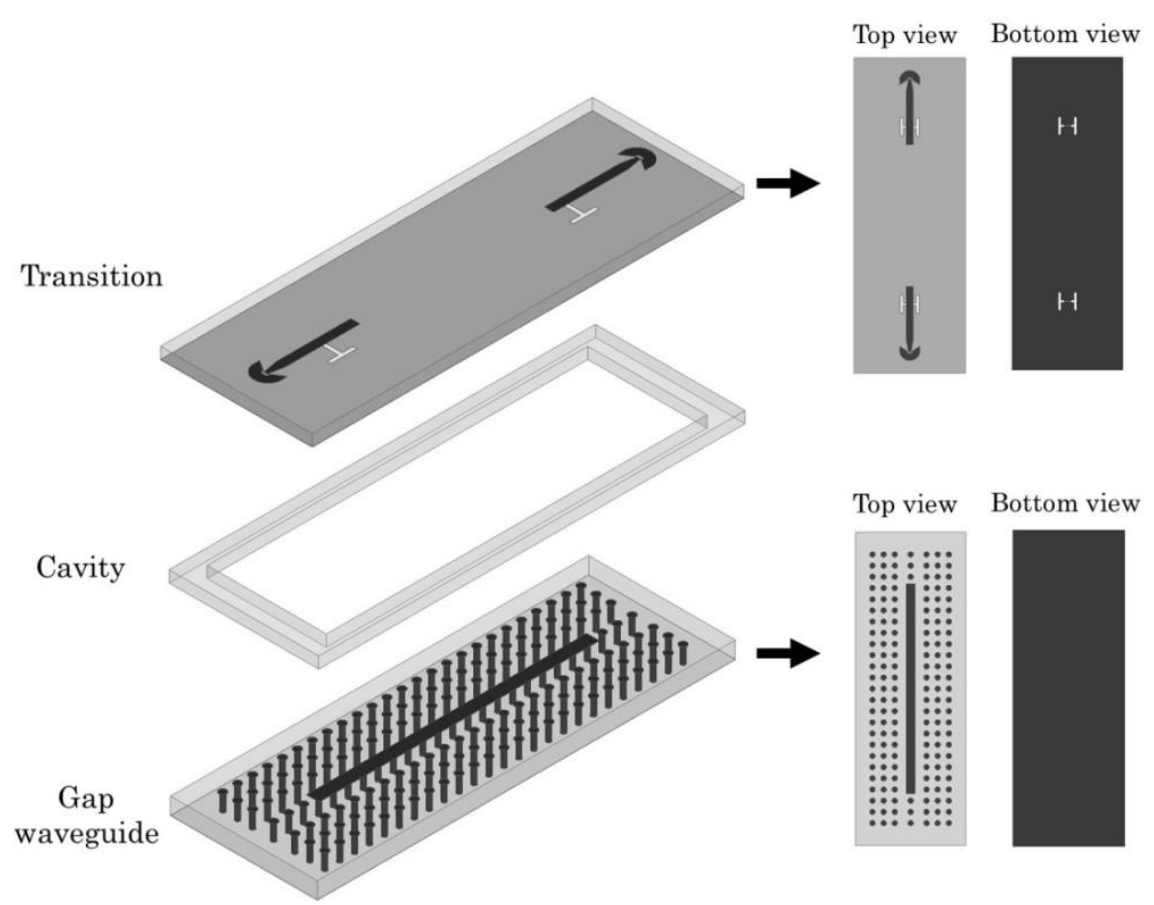

Figure 0.5. Schéma complète du prototype: Transition coplanaire-microruban avec couplage par fente en $\mathrm{H}$, couche pour créer la cavité d'air et gap waveguide.
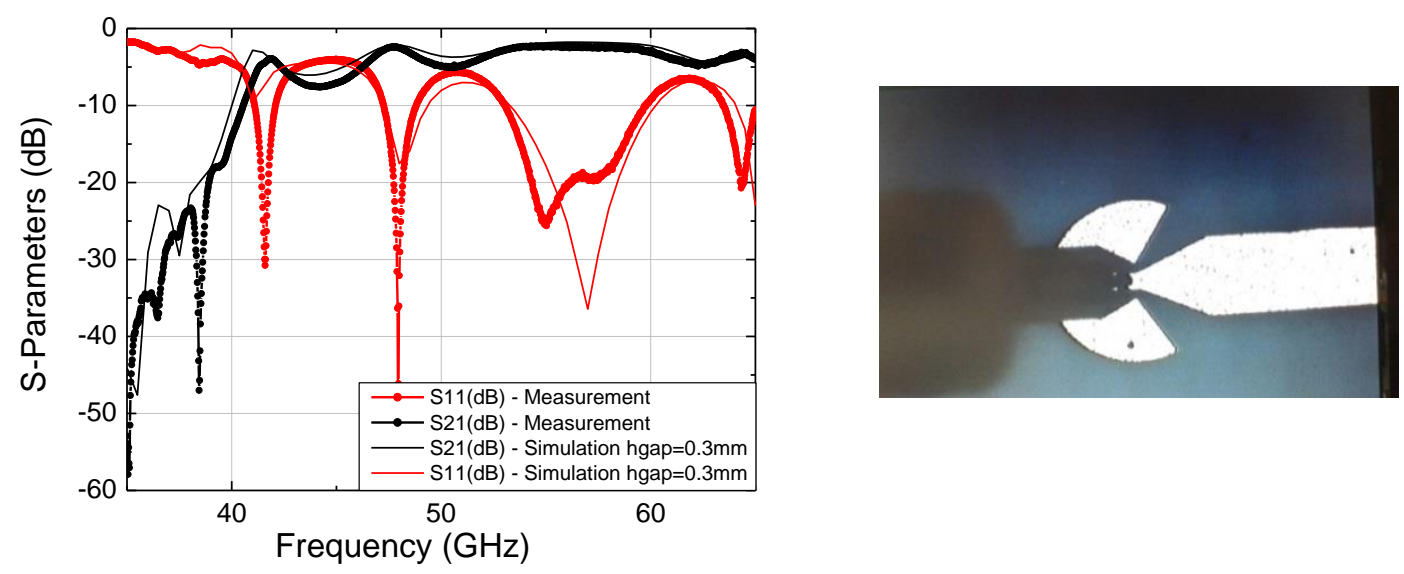

Figure 0.6. Comparaison entre les résultats en théoriques et expérimentaux de la « gap waveguide » fabriqué avec RO4003. 
Pour cette raison, un travail en laboratoire a été fait afin de contrôler les procès de réalisation de cavités. Plusieurs considérations de fabrication ont été expliquées pour le processus de formation de la cavité.

Enfin, le prototype "gap waveguide" a été fabriqué en utilisant LTCC, plus précisément le substrat ESL41110 (Figure 0.7). Des mesures ont montré des problèmes au cours du processus de fabrication. Ces problèmes sont liés à la sérigraphie de l'or et au remplissage. Nous pouvons les résumer aux problèmes suivants : mauvais remplissage des vias, excès de pression dans le processus de pressage et problèmes de désalignement des couches. Pour surmonter les difficultés, il est nécessaire d'améliorer le processus de sérigraphie, car avec la caméra optique de notre système, il est compliqué de voir et d'aligner vias dans l'écran et dans le substrat. Une autre solution consiste à introduire plaque métallique sur le dessus de chaque couche contenant des vias LTCC. Il est également nécessaire d'ajuster la quantité de viscosité de la pâte et numéro de passe. Dans le cas du procédé de pressage, il est intéressant d'étudier une autre solution comme le pressage isostatique en utilisant l'eau chauffée. Cette méthode est plus complexe que la technique uniaxiale, mais présente une déformation inférieure à la densité du substrat.

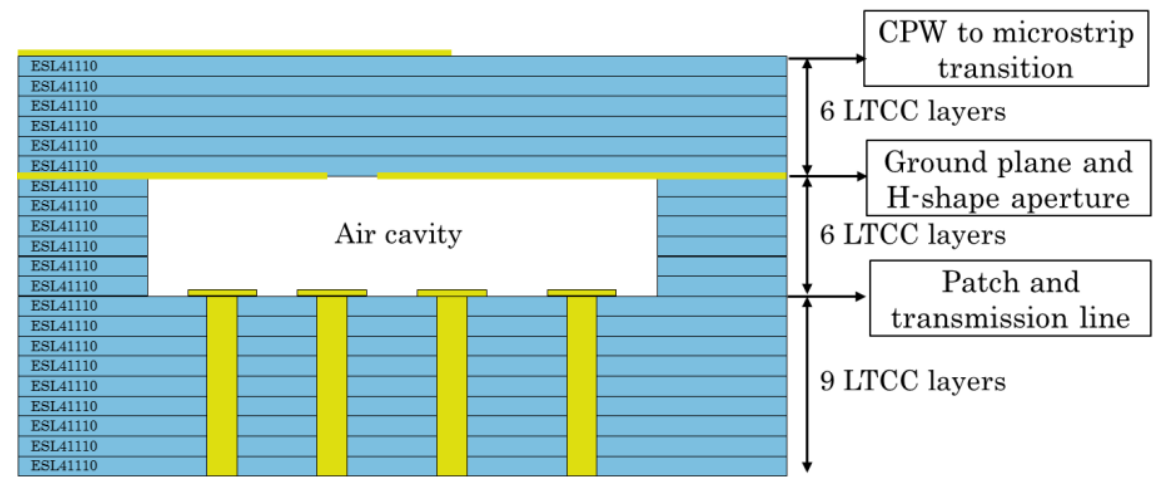

Figure 0.7. Section transversal du prototype "gap waveguide" avec LTCC.

\section{Chapitre 5: Résonateur basé en la technologie gap waveguide pour des applications micro-fluidiques}

Enfin, le cinquième chapitre présente l'application de la " gap waveguide" pour la caractérisation des fluides basée sur des dispositifs résonants. Pour tester les 
performances de notre résonateur, il est comparé avec d'autres technologies en termes de sensibilité. Mesures de résonateurs avec PCB et LTCC sont expliquées.

Dans ce chapitre, nous avons discuté l'intérêt de la technologie "gap waveguide" pour la caractérisation des propriétés électromagnétiques des fluides. Tout d'abord, la conception du résonateur quart de longueur d'onde avec un stub ouvert basé sur la technologie "gap waveguide» est présenté (Figure 0.8). Ensuite, la conception et une comparaison entre gap guide d'onde et SIC ("Substrate Integrated Cavity ») a été réalisée pour mesurer l'impact RF avec l'introduction de deux fluides différents. Cette comparaison montre l'impact des propriétés électromagnétiques du fluide sur la réponse électromagnétique de la structure, avec une variation de la fréquence de résonance. En conclusion, la "gap waveguide" présente une plus grande sensibilité aux changements du fluide parce que l'interaction entre le fluide et le champ électromagnétique a lieu là où le champ électromagnétique est maximal, alors que pour la SIC elle se fait au niveau d'ouvertures et de cavités externes (Figure 0.9). Cette méthode permet de caractériser les liquides avec une bonne sensibilité, par exemple, dans des applications de diagnostic, pour déterminer le niveau de concentration des cellules dans un milieu.

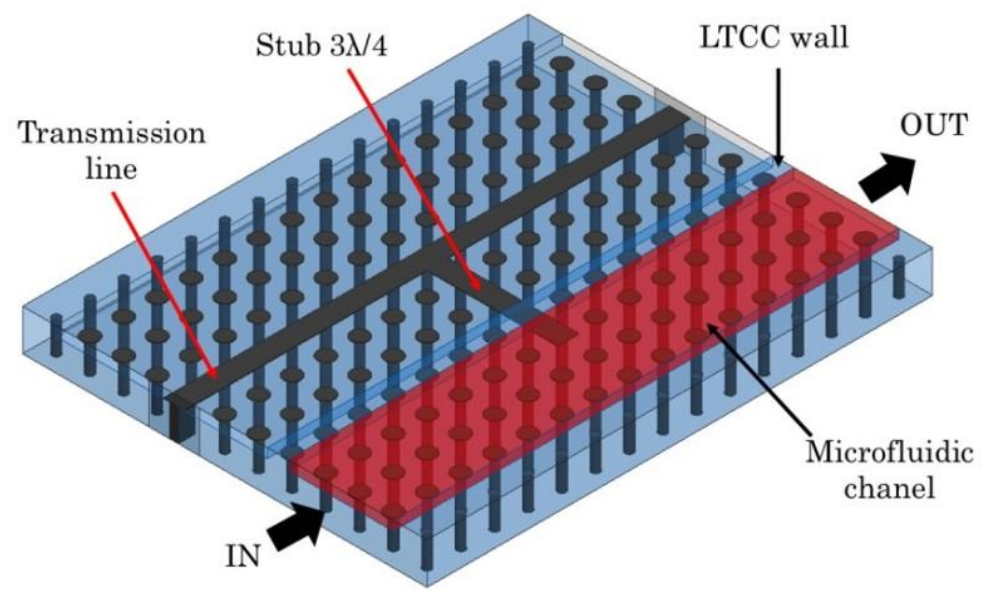

Figure 0.8. Vue 3D du résonateur "gap waveguide" pour la détection des fluides. 


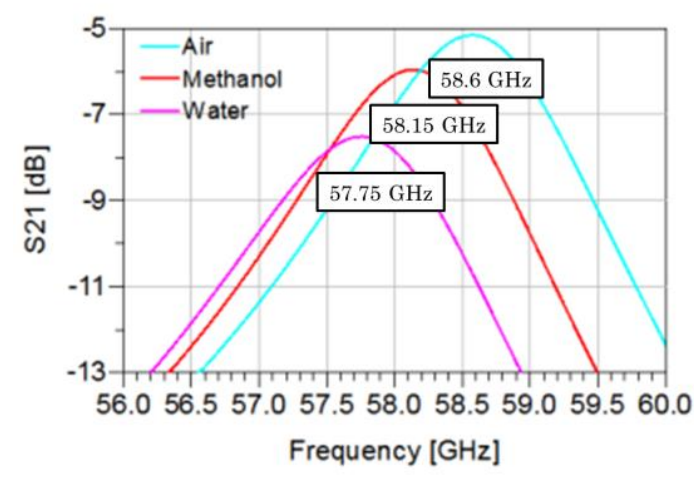

a)

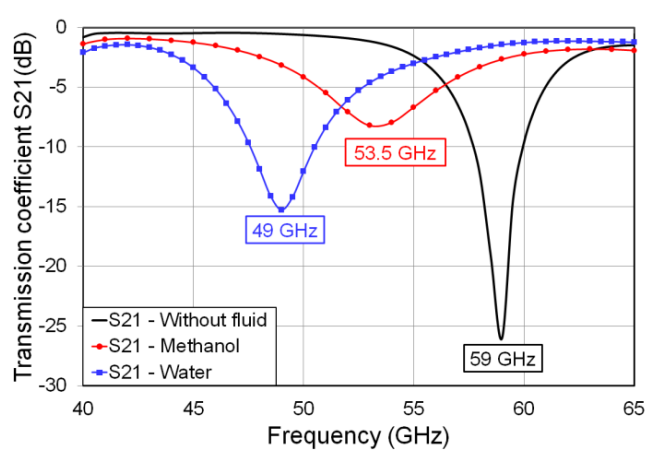

b)

Figure 0.9. Résultats théoriques présentées dans [6] de la structure SIC (a) et avec le résonateur " gap waveguide" (b) avec la cavité vide, et remplie de méthanol et de l'eau.

Ensuite, la conception et le prototype fabriqué du résonateur "gap waveguide " en utilisant PCB et LTCC a été présenté. Les résonateurs basés sur la technologie PCB présentent une bonne concordance entre les valeurs théoriques et les expérimentaux (Figure 0.10). Cependant, une forte dépendance à la hauteur du gap d'air a été observée. En utilisant une technique de circuit imprimé à plusieurs couches, toutes les pièces sont empilées et ce processus peut produire des variations de la hauteur de la cavité. Ces variations ont un impact considérable sur l'adaptation et la transmission entre la transition et le la " gap waveguide». Pour surmonter ces problèmes, LTCC est présenté comme une technologie appropriée dans le but de créer dans une seule étape l'ensemble du module : surface de vias, cavité d'air et transition. Ce prototype a été conçu et présenté, où certains problèmes technologiques ont eu lieu qu'ils ont été définis dans le chapitre 4.

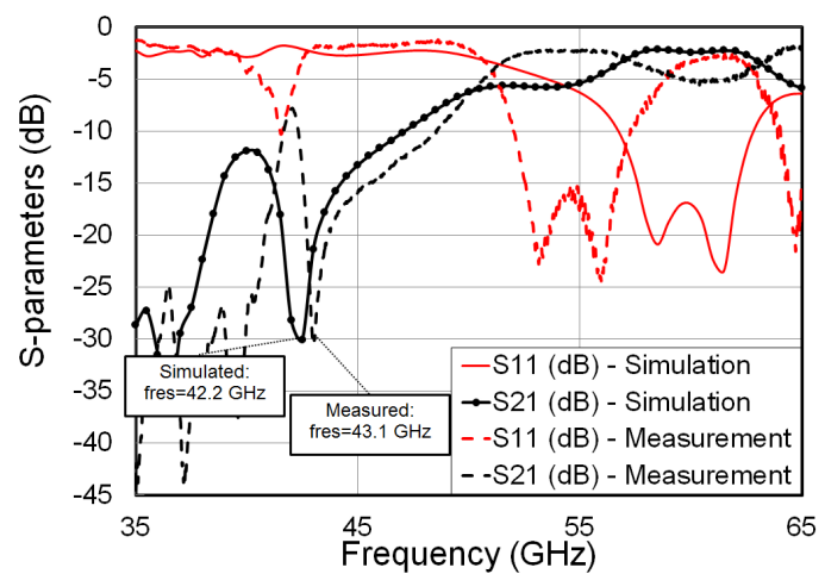

Figure 0.10. Résultats théoriques et expérimentaux du résonateur "gap waveguide" avec RO4003. 


\section{Conclusion générale et lignes futures}

Pour résumer, cette thèse a proposé une nouvelle structure dans la bande d'ondes millimétriques pour la caractérisation des fluides. en améliorant de manière significative la sensibilité des mesures. Le résonateur conçu montre de bonnes performances entre les résultats théoriques et expérimentaux.

Beaucoup de travail peut découler de cette thèse. D'abord, la mise au point de la " gap waveguide" en utilisant la technologie LTCC peut être améliorée. A cet effet, dans le chapitre 4, plusieurs solutions ont été proposées pour améliorer le processus de fabrication LTCC.

En ce qui concerne la caractérisation de fluide, l'étape suivante consiste à remplir les cavités micro-fluidiques avec plusieurs liquides à des mesures pratiques du capteur proposé à $60 \mathrm{GHz}$. Ce point est très important dans la validation complète $\mathrm{du}$ guide d'ondes d'espace pour les applications microfluidiques. La validation des PCB et LTCC devrait comparer les performances et de montrer la biocompatibilité des matériaux différents.

Pour explorer d'autres techniques de fabrication, nous proposons l'utilisation de techniques de PCB combinant avec PDMS canaux pour l'introduction du fluide. En termes de fabrication, cette méthode est simple et rapide et elle peut être compatible aussi avec la fabrication écart de guide d'ondes.

Pour un autre travail, nous proposons également, d'autres types de résonateurs combinant d'autres techniques de fabrication comme par exemple la puce micro-fluidique sur la base liquide accordables déphaseurs de transmission. 
RÉSUMÉ 


\section{INTRODUCTION}

Microfluidic systems have been emerged as a promising technology for molecular analysis, biodefence, and microelectronics. The properties of the microfluidic devices, such as rapid sample processing and the precise control of fluids, have made them attractive candidates to replace traditional experimental approaches. Microfluidic devices are characterized by fluidic channels with dimensions on the order of tens to hundreds of micrometers. Structures with this size enable the integration of lab-on-chip technology, which allows processing miniaturized devices for fluid control and manipulation.

Fluid sensing by microwave sensors based on the RF analysis offers new possibilities for the characterization of mediums by non-invasive methods. Dielectric measurement of fluids is important because it can provide the electric or magnetic characteristics of the materials, which proved useful in many research and development fields, such as molecular biology and medical diagnostics.

Several techniques are available in the frequency domain for analyzing the dielectric properties of liquids and their composition. We are focused on resonant cavity techniques for fluid characterization in the millimeter-wave range. However, these techniques are incompatible with lab-on-chip process due its dimensions. In this context, a new structure called gap waveguide appears as a good candidate to overcome the principal drawbacks of the classical resonant cavities. 
Gap waveguide technology has been presented in the last decade as a promising candidate to address some of the problems faced at millimeter waves with conventional guiding technologies. In this thesis, we are interested in the gap waveguide implementation where the via surface is embedded in a dielectric, with the aim to create cavities and channels in the air gap region above.

This thesis presents the development of the gap waveguide technology in the millimeter-wave band in terms of design and losses. Other conventional technologies are discussed as well, to compare them with the performance in terms of losses of the gap waveguide.

We also present the resonator design based on gap waveguide with the purpose of making the gap waveguide a technology capable of working in the microfluidic sensing domain. In this context, we propose a comparative study between gap waveguide and Substrate Integrated Cavity (SIC) in terms of fluid permittivity characterization at $60 \mathrm{GHz}$. With this purpose, several prototypes have been manufactured with RO4003 substrate and Low Temperature Co-fired Ceramic (LTCC) technology. A work in the LTCC laboratory has been done with the purpose of validating some steps in the LTCC process which are key in the gap waveguide manufacturing, especially those related with the creation of cavities (external and internal) using LTCC materials.

\section{Structure of the thesis}

This thesis is organized as follows:

Chapter 1 presents the motivation and the objectives of this thesis. With this purpose, first, we present the microfluidic applications in the millimeter-wave range. Then, the principal measurement methods for fluid characterization in the microwave range are reviewed. The guiding structures compatible with these methods are also presented. The choice of the guiding structure is based on three characteristics: structure capable at working in the $\mathrm{mm}^{-}$wave band, minimum RF access loss and compatible with the creation of cavities and channels where the electromagnetic field is maximal. The gap waveguide technology has been identified like a promising solution to overcome these three requirements. Once the choice of the guiding structure is made, the manufacturing techniques for microfluidic applications will be analyzed. 
Chapter 2 presents an overview of the gap waveguide technology and the design. An exhaustive study about the stop-band characteristics of the gap waveguide, an impedance analysis and a losses study will be presented. These studies will be developed with the gap waveguide using PCB and LTCC technologies, with the aim to perform cavities in a next step.

In Chapter 3, a comparison between gap waveguide and SIW will be presented. These studies will be done at $170 \mathrm{GHz}$ to reveal the operating limits of both technologies in terms of conductor, dielectric and leakage losses.

Chapter 4 presents the physical implementation of the gap waveguide with PCB and LTCC technologies. Moreover, the feeding transition will be presented. The manufacturing and measurements of both prototypes will be explained. For LTCC, a work at the laboratory will be done with the aim to validate some steps of the LTCC process. In this context, the manufacturing of external and internal cavities will be illustrated.

Finally, Chapter 5 is dedicated to demonstrate the potential of gap waveguide for fluid characterization based on resonant devices. The resonator design and device manufacturing based on the gap waveguide will be explained. For testing the performance of our resonator, it will be compared with other technologies in terms of sensitivity. Measurements of resonators with PCB and LTCC will be explained.

The final conclusion will summarize the contributions of this work and will discuss perspectives and future work. 


\section{Chapter 1}

\section{Motivation and State of the Art}

\subsection{Introduction}

In the last two decades, the interest in microfluidic has grown for biological and chemical sensor applications. These systems offer many advantages over conventional bench top analytical systems such as small sample volumes, portability or disposability [1]. The integration of microwave sensing into microfluidic systems is based on the analysis of the electromagnetic properties of a fluid to obtain information about its composition. Microwave techniques provide powerful tools for characterizing and analyzing liquid samples. The proposal is based on taking advantage of the electromagnetic interaction. The term electromagnetic characterization refers to the full knowledge of the electromagnetic properties (complex permittivity and permeability) of a material versus frequency.

This chapter describes the state of the art of the different parts forming this thesis. First, we present the interest of microfluidic applications. Then, we justify the interest of microfluidic applications in the millimeter-wave band. After, the state of the art of the measurement methods used fluid sensing in the millimeter wave range is studied. Next section presents a review of the classical guiding structure used for liquid characterization at microwave frequencies. And finally, 
once the guiding structure has been chosen, the operating frequency range and the measurement method have been defined, we present a state of the art of the several manufacturing techniques for microfluidic devices.

\subsection{Microfluidic applications: microwave fluid sensing}

Permittivity measurements can give valuable information on liquid and biological systems. As the measurement frequency is increased, we can observe various relaxation phenomena that are specific to the given materials [2], which could potentially lead to the electromagnetic identification of unknown samples. Determining the dielectric properties of biological samples is also necessary for the development of new microwave or millimeter-wave based medical treatments and diagnostics, and could also be applied to investigate the effects of electromagnetic radiation on cells at the cellular level.

Liquids can be characterized as dielectric materials. Dielectric material properties are permittivity and permeability that depend on the frequency. The main mechanisms that contribute to the permittivity of a dielectric material are conduction, dipolar relaxation, atomic polarization and electronic polarization. At low frequencies $\varepsilon$ " is dominated by the influence of ion conductivity. At microwave frequencies, the variation of permittivity is mainly due to dipolar relaxation.

Originally, fluid characterization has been investigated at first time to allow material modeling for dosimetry and more recently for the development of microwave fluid biosensors.

\subsection{Increasing frequency for liquid characterization}

As it can be seen in the previous section, the microwave range offers the possibility to characterize the fluids in terms of effective permittivity and $\tan \delta$. With this purpose, several methods have been presented in the literature [3]. Usually the maximum frequency range for the permittivity characterization of fluids is up to 20 or $40 \mathrm{GHz}$. These frequencies allow integrating classical guiding structures as coplanar or microstrip transmission lines and resonant cavities. Nevertheless, the results presented in [4] show an interest to increase testing frequency for the permittivity characterization of fluids. In this reference mentioned, the dielectric constant and the conductivity are measured up to 20 $\mathrm{GHz}$ for different glucose levels. Measured dielectric constant and conductivity is 
represented in Figure 1.1. We can see that at $20 \mathrm{GHz}$, the permittivity and the conductivity is more sensitive for several glucose concentrations.

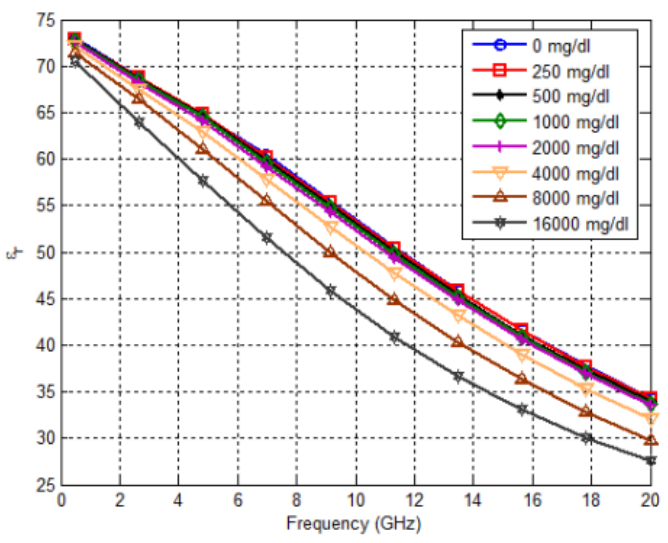

a)

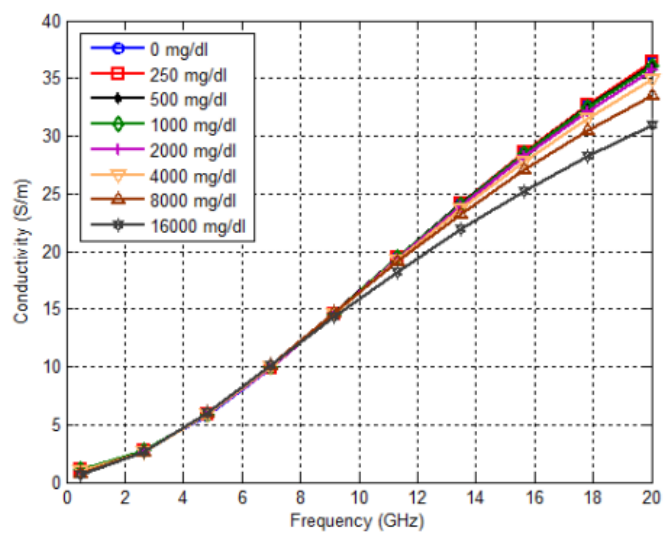

b)

Figure 1.1. Measured a) dielectric constant b) conductivity from $500 \mathrm{MHz}$ to $20 \mathrm{GHz}$ for various glucose levels presented in [4].

Another example is presented in [3], where the conductance of alcohol-based aqueous solutions and for concentration of living lymphoma cells is presented as a function of the frequency (Figure 1.2). As can be seen, the stronger contrasts occur at $40 \mathrm{GHz}$. Therefore an improvement in the detection of varying concentration of cells can be achieved at the frequency with respect to lower ones.

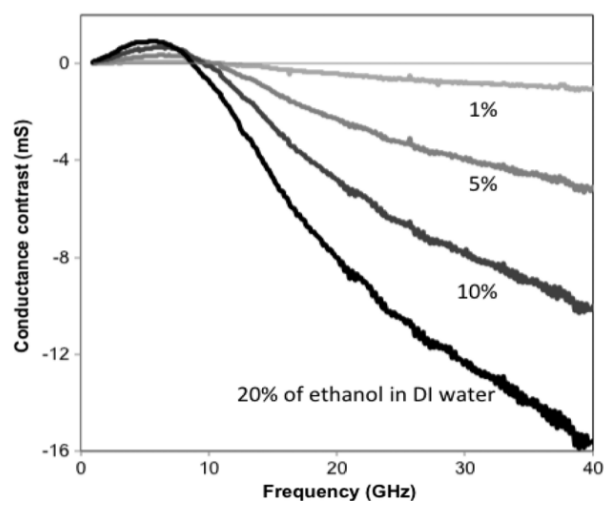

a) Contrast spectra of the conductance of alcohol-based aqueous solutions

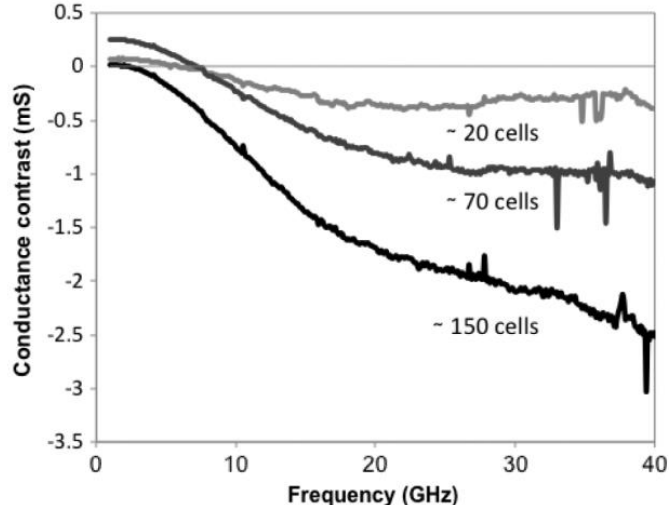

b) Conductance for different concentration of living RL lymphoma cells in their biological culture medium.

Figure 1.2. Results presented in [3]. 
Both examples show that there is an interest for fluid characterization in the mm-wave band, due to strong variation levels of the sensitivity. In this context, lab-on-chip technique appears as a good candidate for the miniaturization of the devise in the mm-wave band.

We can summarize our objectives as:

1. Work in the mm-wave band.

2. Low RF access losses.

3. Maximum interaction between the fluid and the electromagnetic propagation.

4. Guiding structure capable to integrate cavities and channels.

\subsection{Measurement methods}

In this section, we present a review of the measurement methods existing in the literature for fluid sensing in the microwave frequency range. Microwave measurement methods for material characterization are divided into two types, resonant and non-resonant approaches. Resonant methods give more accurate knowledge of dielectric properties over a limited frequency range or a single frequency, while the non-resonant methods give a general knowledge of electromagnetic properties over a frequency range. Non-resonant methods based upon reflection or transmission/reflection methods.

\subsubsection{Reflection methods}

Reflection methods are based on the analysis of the reflection scattering parameters of the devices. By measuring the reflection coefficient, it is possible to detect the electromagnetic properties of the material under test (Figure 1.3). We can distinguish two reflection methods based on: open-ended coaxial probe and waveguides.

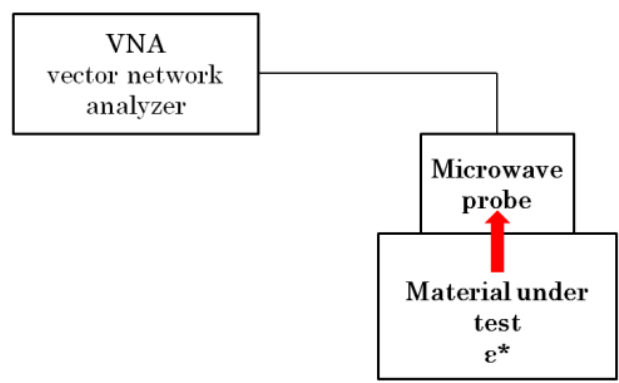

Figure 1.3. Illustration of the principle of the reflection method. 


\subsubsection{Open ended coaxial probe}

The open-ended coaxial probe is a cut off section of a coaxial transmission line. The material is measured by immersing the probe into a liquid or pulling it in contact with the flat face of a solid material. This method is one of the most employed methods for measurement of lossy materials at high frequency. Coaxial line uses a metallic probe, which senses reflected signal (phase and magnitude) from the fluid under test. The basis of the operation is represented in Figure 1.4. This broadband method is characterized by its simplicity and the non-invasive analysis of the samples.

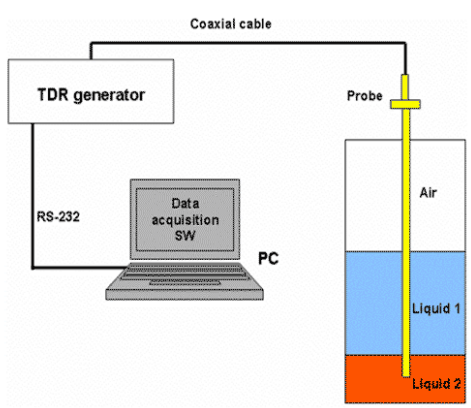

Figure 1.4. Principle of operation of the open ended coaxial method.

The coaxial probe method is basically a modification of the transmission line method. It uses a coaxial line, which has a special tip that senses the signal reflected from the material. The tip is put in contact with the substance by touching the probe to a flat flake of a solid or by immersing it in a liquid. The reflected signal is related to the dielectric properties of the substance. Moreover, it is possible to measure the dielectric properties over a range of frequencies (up to $40 \mathrm{GHz}$ ) with limited accuracy particularly using materials with low values of absolute permittivity.

For example, in [5], a method for broadband measurement of liquids using the open-ended coaxial method is presented. Measurements have been done in the range from $50 \mathrm{MHz}$ to $3 \mathrm{GHz}$. This system allows characterizing the complex permittivity of the sample material based on an inverse problem to coincide with the calculated input impedance and the results of the input impedance measurements. Figure 1.5 shows the open-ended coaxial line method and the measurement system. 


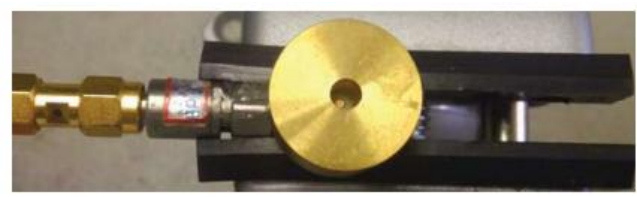

a) Measurement jig.

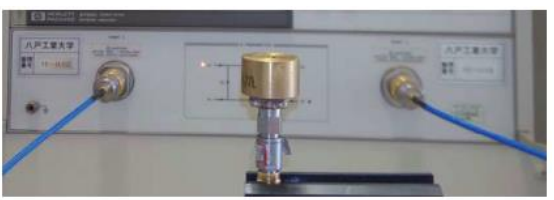

b) Measurement setup.

Figure 1.5. Open-ended coaxial line reflection method presented in [5].

A system for measuring the properties of liquids and biological tissues over a frequency range from $50 \mathrm{MHz}$ to $2.6 \mathrm{GHz}$ using an open-ended probe is presented in [6]. The uncertainties in the measurements are less than $10 \%$.

Another example is presented in [7]. The schematic of the proposed sensor is based on dielectric probe measurement tank and is shown in Figure 1.6. This sensor aims at estimating the tissue properties over a sensing volume of 2 to 3 $\mathrm{mm}$. Measurements have been performed up to $8 \mathrm{GHz}$.

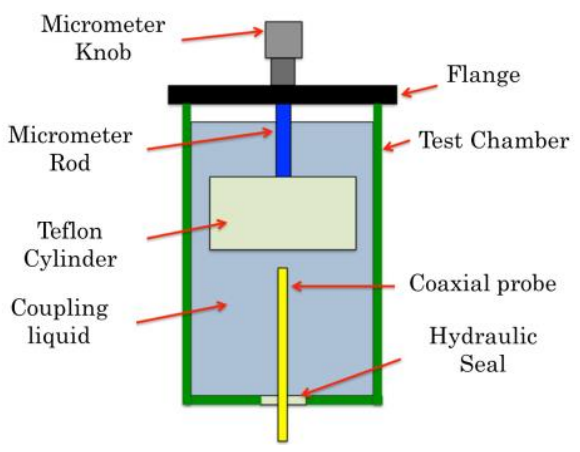

a) Schematic of the experimental test configuration

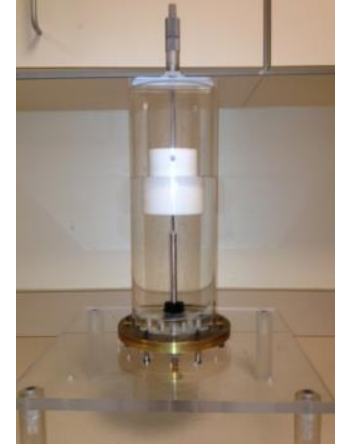

b) Photograph of the physical test configuration

Figure 1.6. Schematic and physical implementation of the sensor proposed in [8].

The uncertainty levels of the complex permittivity measurement are higher than other techniques that we explain in this chapter. Moreover, calibration methods can be delicate and long duration. For these reasons, the dielectric characterization using coaxial probes due its dimensions is a method incompatible with lab-on-chip techniques.

The disadvantage of the coaxial line reflection method is the limited accuracy, under some conditions when compared to other methods like the transmission line method and resonator method. The open coaxial probe device requires strict 
operating conditions: cable stability and especially, control of the air gap between the probe and the sample surface.

\subsubsection{Waveguide probes}

Waveguide probe is a section of a metal waveguide with rectangular cross-section excited above the cut-off frequency. This section is shorted from one side and from another side the material under test is placed. A system based on the waveguide permittivity characterization is presented in [8]. This system deals with the complex permittivity measurement of biological tissues and is shown in Figure 1.7. The measurement method is non-destructive and non-invasive based on reflection coefficient measurement. The properties of the prototypes have been studied in the frequency range from $300 \mathrm{KHz}$ to $3 \mathrm{GHz}$ in [9].

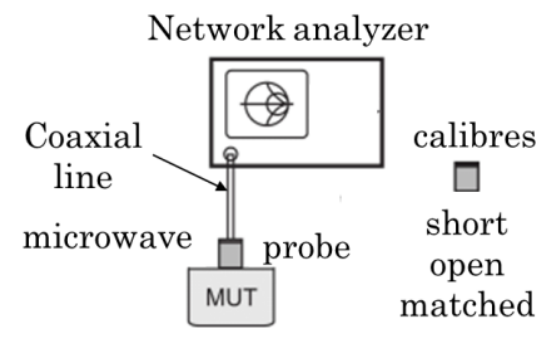

a) Measurement system

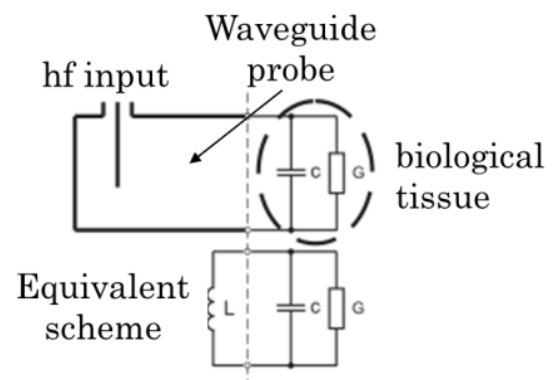

b) Equivalent circuit probe

Figure 1.7. Measurement system presented in [8].

In the reflection methods, coaxial probes are more used in the literature than waveguides due to its smaller size and because waveguide probes are more limited in bandwidth.

\subsubsection{Transmission line method}

With transmission line method, the sample is placed inside a shielded transmission line and both reflection and transmission coefficients are measured. The values of $\varepsilon$ ' and $\varepsilon$ " are derived from transmission line theory, by measuring the phase and amplitude of microwave signals reflected from or transmitted through a sample of material. This method based on coaxial, waveguide probe and planar technology transmission lines. 


\subsubsection{Coaxial transmission line}

In the coaxial transmission line, the fluid under test is inserted between the two conductors of the coaxial probe. In [9], the complex permittivity of a liquid phantom with high-permittivity and high-loss has been measured by the transmission line method using a coaxial line (Figure 1.8). Measurements have been done between 0 and $3 \mathrm{GHz}$. Results show that the measurement error of the complex permittivity due to filling error becomes $2.3 \%$ at $2 \mathrm{GHz}$.

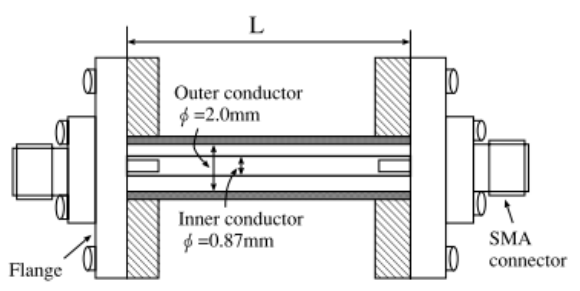

a) Treatment device consisting of coaxial line

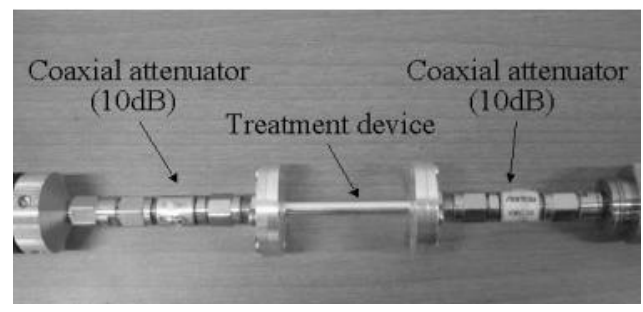

b) Measurement setup

Figure 1.8. Transmission line method using coaxial line presented in [9].

Coaxial probes used as the transmission method show more accuracy than coaxial probes in reflection, especially for frequencies $\mathrm{f}>500 \mathrm{MHz}$ as it is proposed in [10]. This system aims to overcome the accuracy problems of the conventional open-ended coaxial line technique due to the sensitivity of the method and to small inhomogeneities in the measured liquid.

However, coaxial probes in transmission are a process more complicated than the open-ended coaxial probe in reflection. Coaxial probes need to introduce the fluid in the dielectric part between the two conductors. Moreover, they are nocompatible with lab-on-chip techniques due its dimensions and metallic interconnects.

\subsubsection{Waveguide transmission lines}

With the waveguide transmission line method, the sample is placed in a section of the waveguide. Measurements of the complex scattering parameters of the two ports are performed. For a good dielectric measurement, maximum interaction between the material under test and the electric field is required. An example of the measurement apparatus is presented in Figure 1.9 [11]. 


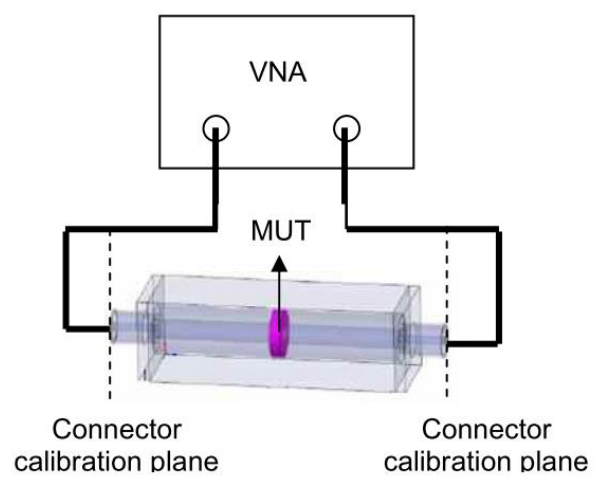

Figure 1.9. Transmission method for material characterization using waveguide transmission line.

In [12], a method to determine the dielectric constant of a thin dielectric slab placed in a waveguide is presented. The proposed system is shown in Figure 1.10. It overcomes the practical problem in the reflection method where the sample must be placed exactly at the waveguide flange. Material under test is Teflon and measurements have been done up to $5.8 \mathrm{GHz}$.

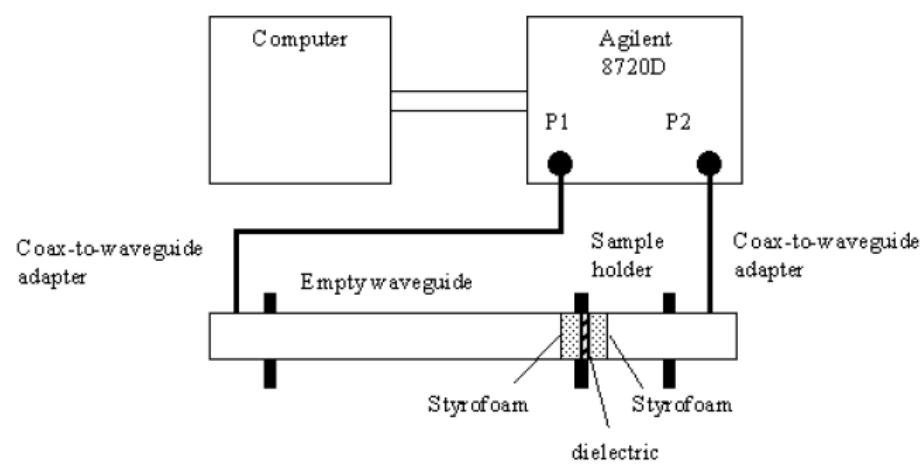

Figure 1.10. Measurement system presented in [12].

In [13], a technique based on transmission/reflection measurements for the determination of the complex permittivity of materials partially filling the cross section of a rectangular waveguide has been described. It demonstrates that the permittivity accuracy obtained using partially filled waveguides is higher than that obtained in completely filled structures. Nevertheless, this method presents limitations in terms of the design of the fluidic structure under test. It is more appropriate for solid characterization. 
The issue of waveguide probes is that they are devices for narrow frequency bands due to the existence of different propagation modes. Moreover, in the microwave range, the liquid volume is higher than the other methods and waveguide probes are incompatible with the lab-on-chip technology.

\subsubsection{Planar transmission lines (coplanar lines)}

Historically, planar transmission lines (coplanar and microstrip lines) has been used for the complex permittivity measurements of dielectric materials. Moreover, the development of the microfabrication techniques and the microfluidic technology allows manufacturing these devices for fluidic characterization.

For example, in [14], a coplanar waveguide transmission line with polydimethylsiloxane (PDMS)-based microfluidic channels is presented to extract the distributed circuit parameters of the fluid-loaded transmission line using the transmission-line model (Figure 1.11). The complex permittivity of the fluid is determined by the distributed capacitance and conductance of the fluid-loaded transmission line segment. Measurements allow determining the frequencydependent complex permittivity of nanoliter fluid volumes for frequencies up to $40 \mathrm{GHz}$.

(a)

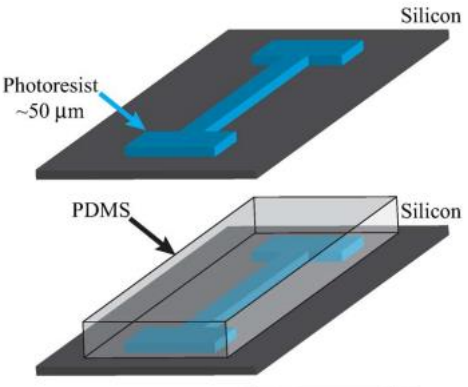

(c)

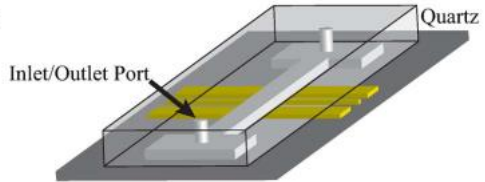

a) Schematic of the planar device integrating the microfluidic channel.

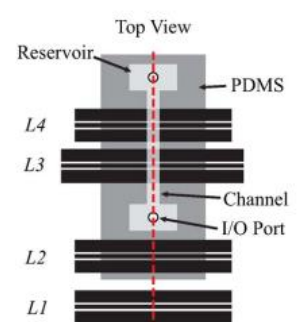

(a)
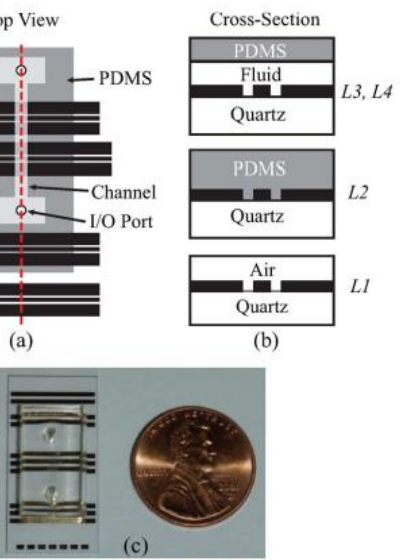

b) Integrated microelectronic microfluidic test structures

Figure 1.11. Measurement system based on microfluidic channels embedded in coplanar transmission lines proposed in [14]. 
Another interesting example is presented in [15], where the dielectric properties of fluids are obtained with coplanar waveguide using the multiline method. The multiline technique consists of the measurement of the scattering parameters of two lines with a known length difference. Two channels are placed in the top of the coplanar lines. The multiline technique allows characterizing the propagation constant, and, as a consequence, the complex permittivity of the fluid under test inside the channels. Analysis has been done up to $5 \mathrm{GHz}$.

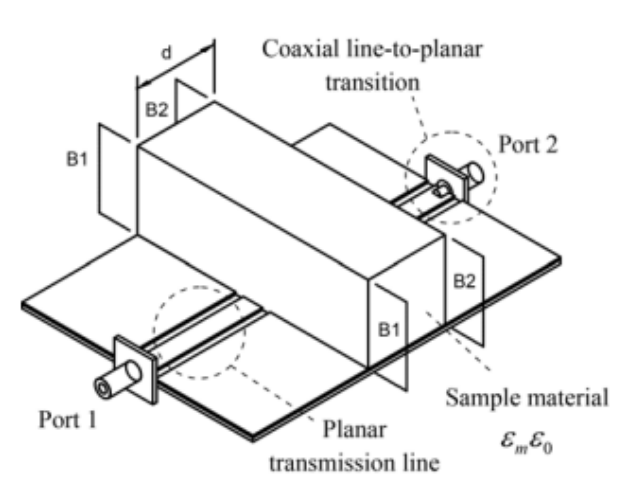

a) Schema of a planar transmission-line sensor.

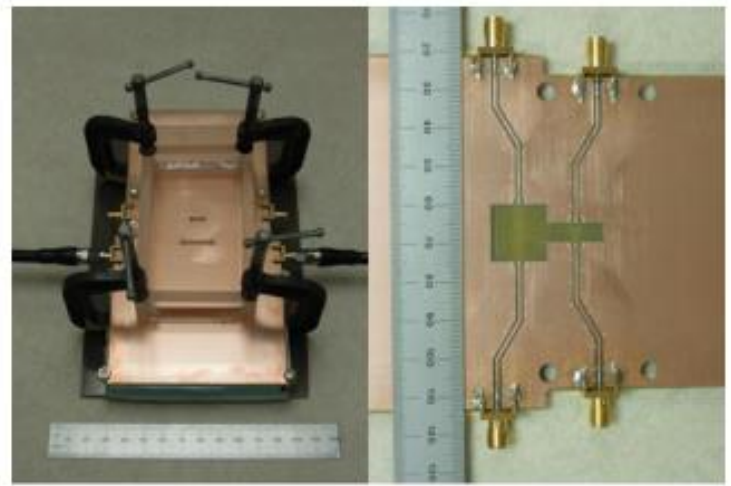

b) Photograph of fabricated sensor: Top side with liquid sample and bottom side with the feed lines.

Figure 1.12. Planar transmission line sensor illustrated in [15].

Yet another interesting implementation was developed by T. Chen et al. in [3] In this work, the fluidic sensor is composed by a coplanar waveguide and an interdigitated capacitor (IDC) with a microfluidic channel to confine the nanoliter-range liquid. This microfluidic channel has been done with PDMS techniques. In the Figure 1.13, the schematic and a photograph of the manufactured prototype is shown. The characterization of liquids has been performed up to $40 \mathrm{GHz}$. The advantages of this device are that it uses small volume of liquid solutions and the in-culture medium analysis of cells, assures a noninvasive impact on living cells. 


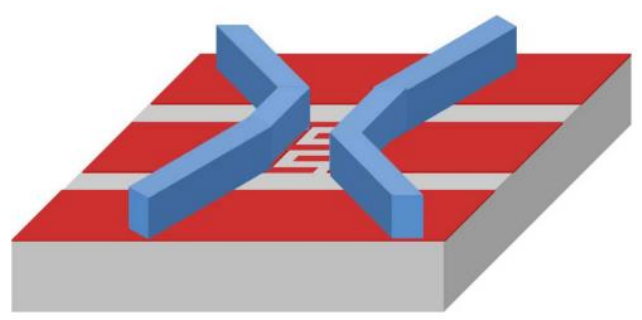

a) Schematic of the IDC with a narrowed microfluidic channel placed on top

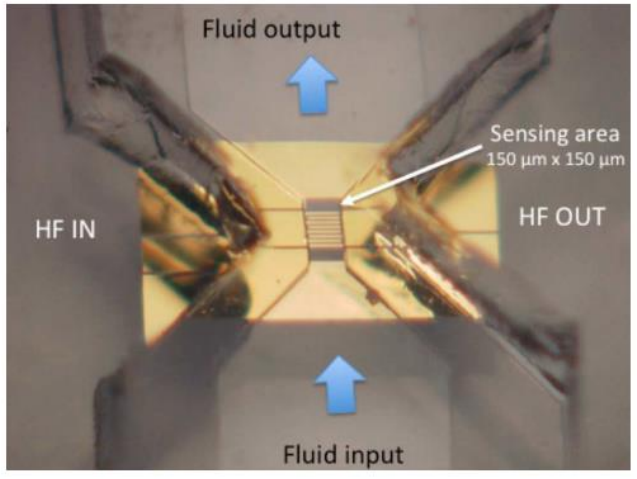

b) Photograph of the fabricated microfluidic IDC with a PDMS channel

Figure 1.13. Proposed coplanar sensor presented in [3].

\subsubsection{Resonant transmission lines}

Typical microfluidic systems acting as a sensor are the so-called microwave resonator. They are theoretically more sensitive than the non-resonant methods, which allow characterizing the fluid over a large frequency band. Inside of the resonant transmission lines, microwave cavities are widely used for characterizing the properties of materials. Different configurations of microwave sensors have been suggested for detecting various chemical ingredients in liquids both organic and inorganic. They are based on the interaction of propagating or resonating modes with solutions under test. Both, waveguides and microwave cavity resonators can be used for practical manufacturing of sensors [16].

Nevertheless, structures based on resonant methods can determine the complex permittivity of the fluid under test for only one determined frequency, the resonant frequency.

\subsubsection{Resonant cavities}

Cylindrical resonant cavities composed by cylinders with metallic boundaries that allow confining the electromagnetic field inside the cavity, are the most popular of the resonant methods. It is very convenient for dielectric measurements and loss tangents over a wide range. Measurement method is based on perturbation technique, which consists of analyzing the Maxwell's equations to derive expressions for the resonant frequency shifts. 
Figure 1.14 shows an example of a cylindrical cavity resonator with the sample located in the middle of the resonator.

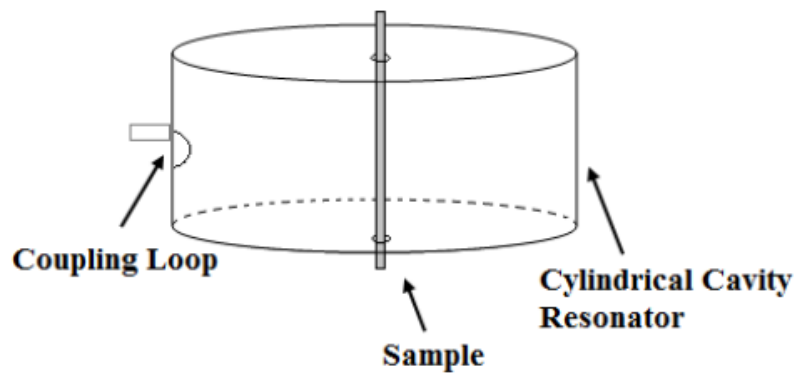

Figure 1.14. Cylindrical Cavity resonator at the TMono mode.

In [16] a microwave resonator sensor for measuring both organic and inorganic ingredient concentrations in water solution was developed and tested (Figure 1.15). It provides high accuracy measurements in the range of $0-5 \%$ of concentration. Measurements have been done at around $1.5 \mathrm{GHz}$.

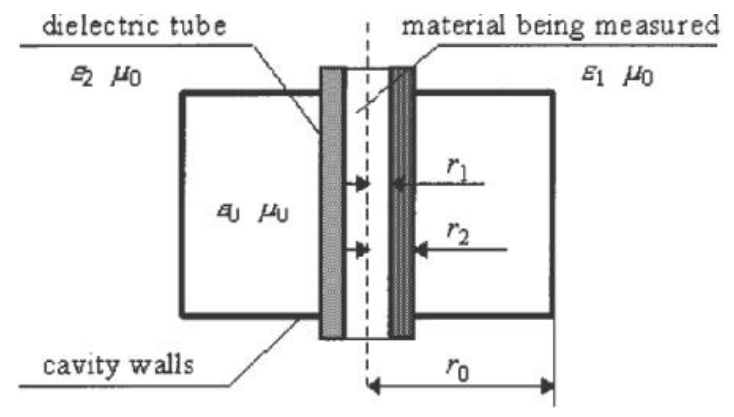

a) Cylindrical resonator with a sample placed on the axis.

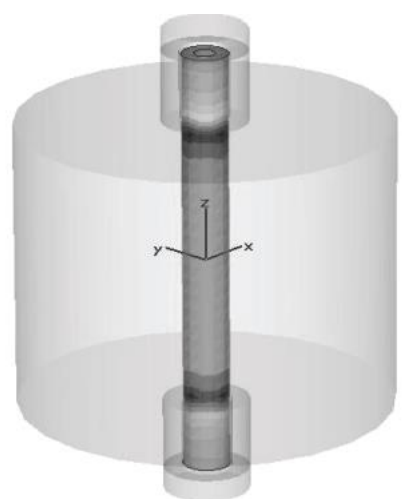

b) Glass tube with liquid under test.

Figure 1.15. Cylindrical resonator for sample characterization presented in [16].

\subsubsection{Dielectric resonator}

As in the case of the resonant cavities, dielectric resonators have a high Q-factor that enhances more measurement accuracy.

In [17], a sensor based on cylindrical dielectric resonators acting on whispering-gallery modes is presented. Whispering-gallery modes are superior order mode propagated around the main rotational axis of the dielectric 
resonator. The electromagnetic field is confined inside the dielectric disk. Figure 1.16 shows the manufactured dielectric sensor.

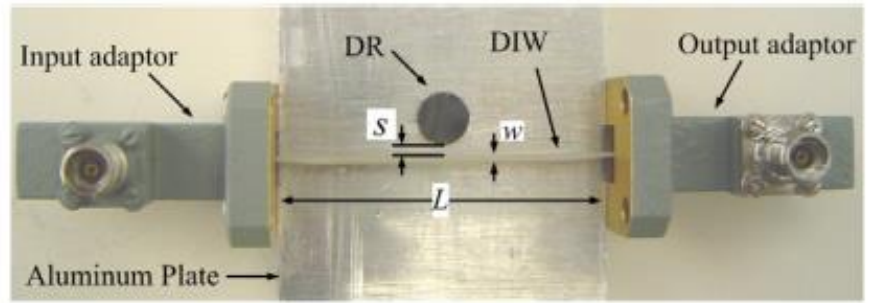

Figure 1.16. Prototype of the dielectric disk resonator proposed in [17].

For the purpose of fluidic applications, the simplest characterization consists of the interaction between the fluid and the electromagnetic field by means of a liquid drop on the upper side of the resonator as for example in [18]. The structure operates between 85 to $110 \mathrm{GHz}$.

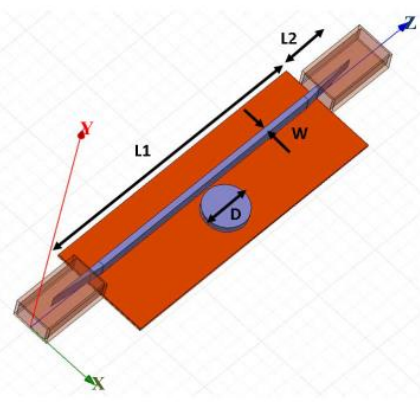

a) General structure of the sensor
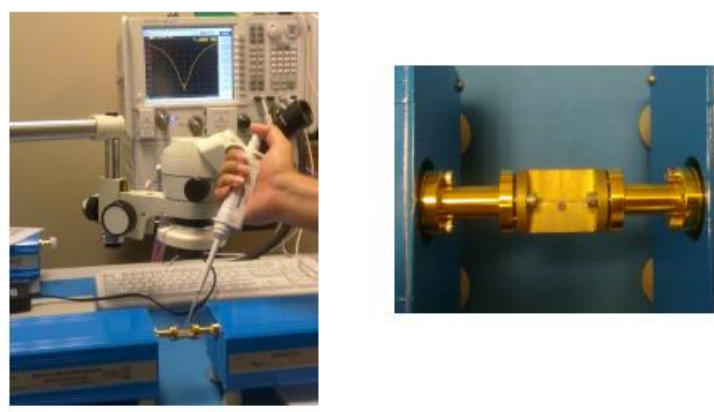

b) Experimental setup

Figure 1.17. Dielectric sensor presented in [18].

Principal advantage of dielectric resonators is the measurement accuracy ( $0.5 \%$ for $\varepsilon$ ' measurement). However, the disadvantages of these resonators are:

- Problems with the positioning of the fluid sample in the resonator.

- Presence of evaporation phenomena.

- High volume that make them incompatible with lab-on-chip techniques.

\subsubsection{Planar lines resonator (incorporated in coplanar and microstrip lines)}

An example based on the planar line resonator method is presented in [19]. In this work, an enclosed microstrip resonator with a slot in the ground plane has 
been designed and fabricated for complex permittivity measurements. The maximum relative error is $8.6 \%$ for a specified permittivity. For lower permittivities, the relative error in measurements is less than $4 \%$.

Another example is presented in [1] where a single-spiral microstrip sensor has been presented. With this method, changes in transmission measurements correspond to changes on the blood glucose level measurements. It is presented as a good candidate for glucose measurements due its non-invasive behavior.

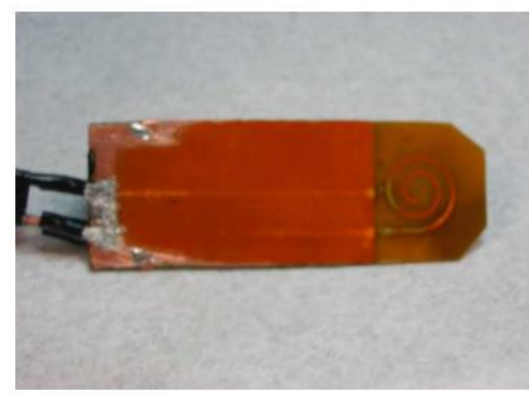

a) Close-up of spiral sensor

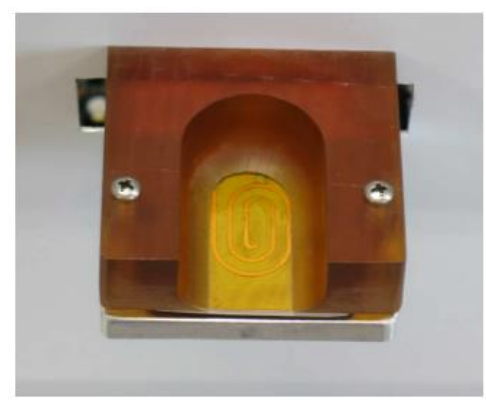

b) Close-up of spiral microstrip with plastic thumb-guiding fixture

Figure 1.18. Non-invasive glucose sensor proposed in [1].

In [20], the microfluidic sensor presented allows the accurate complex permittivity characterization of aqueous solutions (Figure 1.19). It is compatible with lab-on-chip technique due its dimensions and the volume of fluid samples. Measurements are presented with DI (deionized) water/ethanol mixtures in the frequency range up to $20 \mathrm{GHz}$. The S21 parameters obtained in [20] for deionized water/ethanol mixtures is presented in Figure 1.20. Results show as increasing the ethanol concentration, frequency and sensitivity varies.

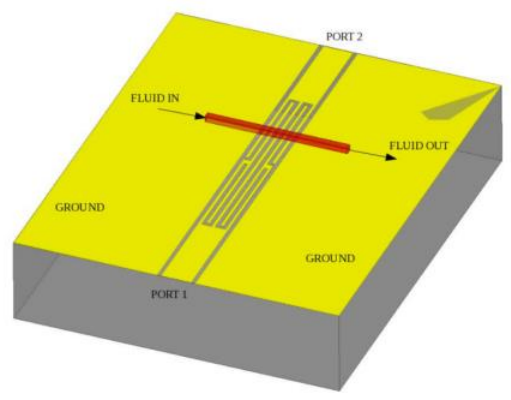

a) Schematic view of the RF coplanar

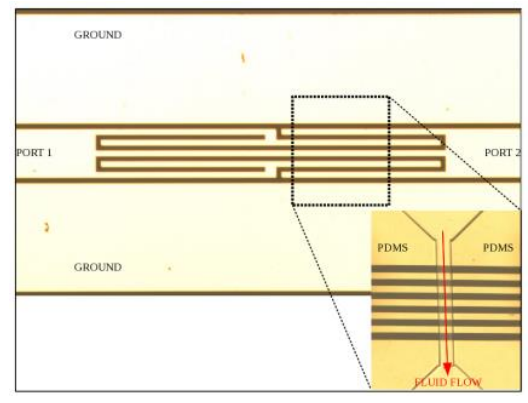

b) Fabricated prototype. resonator with a microfluidic channel on top.

Figure 1.19. Coplanar resonator presented in [20]. 


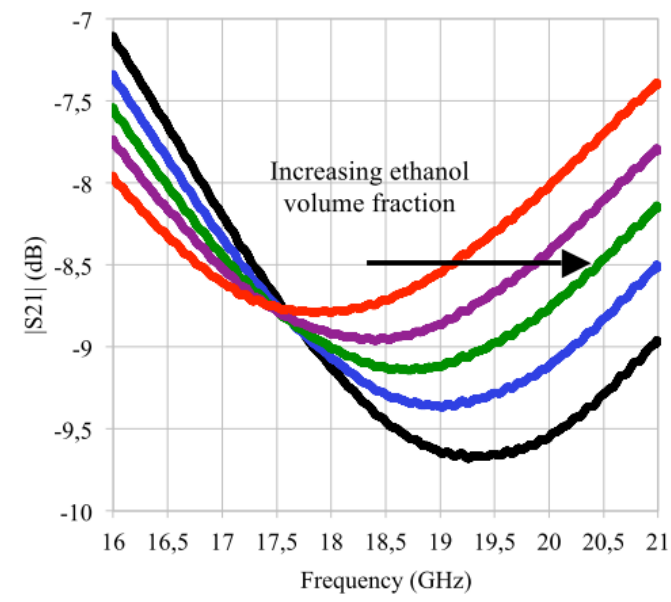

Figure 1.20. S-parameters for 5 ethanol/DI water mixtures at several volume fractions presented in [20].

In [21], a miniaturized transmission-line sensor based on CPWs for characterizing biologically relevant liquids is presented. The biological samples are inserted in a microfluidic channel and are used between $850 \mathrm{MHz}$ and 40 $\mathrm{GHz}$ to detect the broadband permittivity of liquid samples.

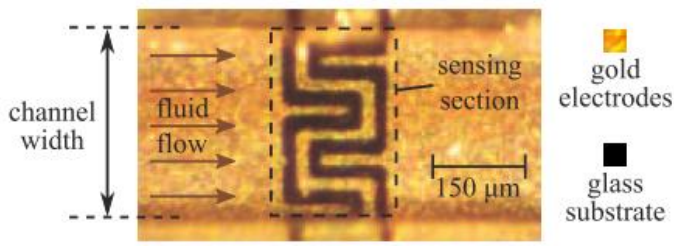

(a) Sensing section of miniaturized transmission-line sensor.

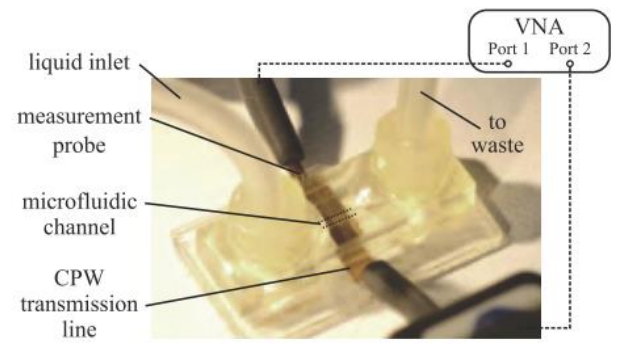

b) Measurement setup of transmission-line sensor with microfluidic channel.

Figure 1.21. Transmission line sensor proposed in [21].

In [22] a microfluidic tunable stop band device has been presented at $25 \mathrm{GHz}$ with SU-8 photoresist (Figure 1.22). To reduce the losses of the SU8 ( $\tan \delta=0.04$ ), they create an air gap channel inside the substrate (Figure 1.23). Liquid under test is deionized water and the filter response by introducing the fluid is modified by more than $20 \%$. 


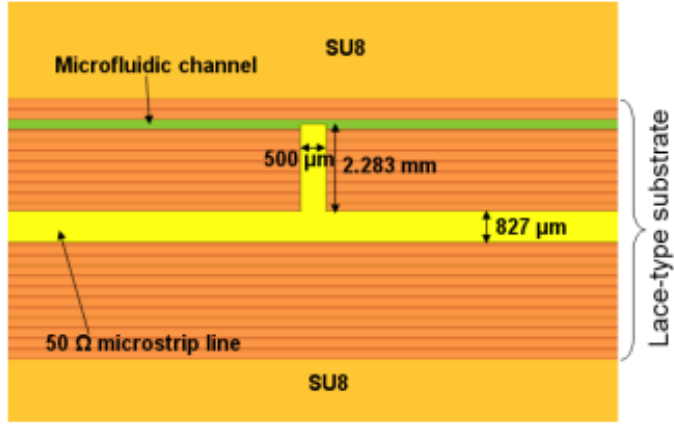

a) Schematic of the microstrip stub resonator.

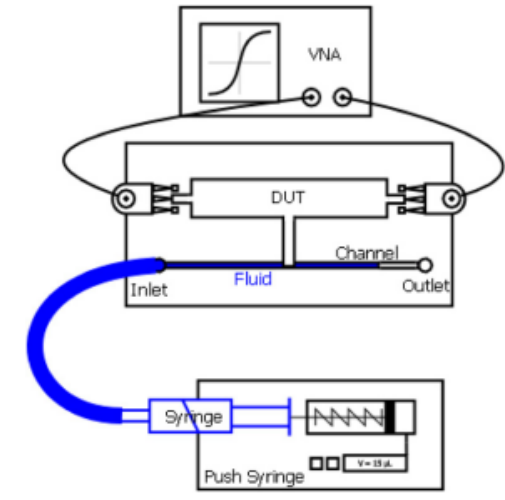

b) Schematic drawing of the set-up.

Figure 1.22. Microstrip stub resonator presented in [22].
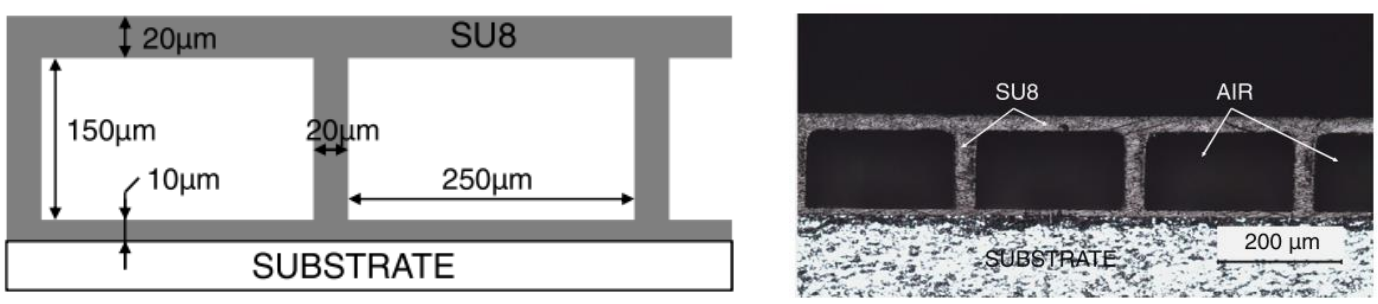

Figure 1.23. Cross section of the microstrip substrate (designed and manufactured, respectively).

\subsubsection{Conclusion}

The advantages, disadvantages and properties of each method: reflection (RF), transmission (TR) and resonant (RS) are summarized in Table 1.1.

The state of the art of several measurement methods for fluid characterization shows that resonant cavities are the best solution in terms of accuracy in the measurement. However, they are bulky in the $\mathrm{mm}^{-w}$ wave band and noncompatible with lab-on-chip techniques. Another solution that shows good performances is the use of planar transmission lines. With this method, the accuracy is reduced, but they are smaller and with less volume of fluid required. Moreover, it is compatible with the compactness leading to minimize fluid volumes in the mm-wave band.

As we will see at the end of this chapter, our analysis will be based on the resonant cavities concept integrating the miniaturization of devices as in the case of planar transmission lines. 
Table 1.1. State of the art of the measurement methods of fluids at the microwave range.

\begin{tabular}{|c|c|c|c|c|c|c|c|c|}
\hline 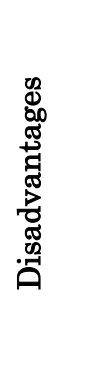 & 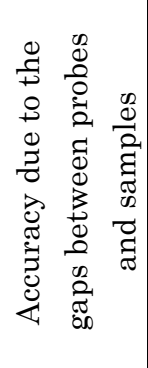 & 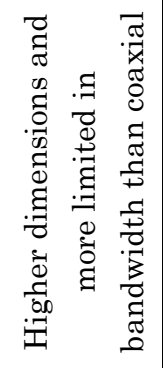 & 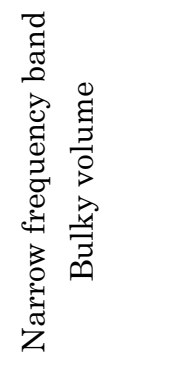 & 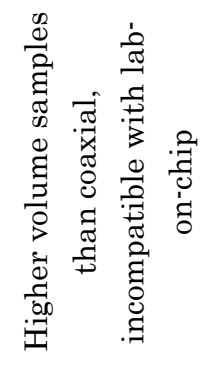 & 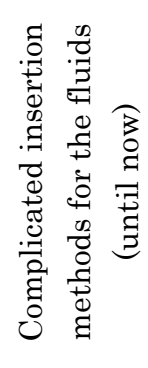 & 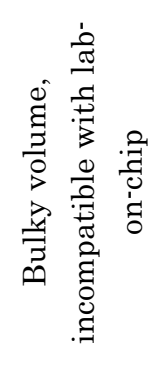 & 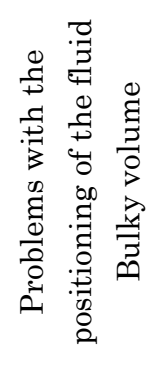 & 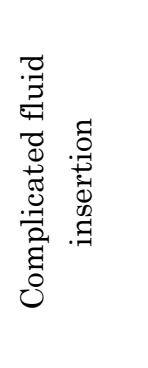 \\
\hline 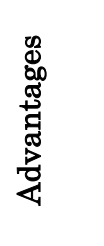 & 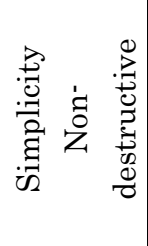 & 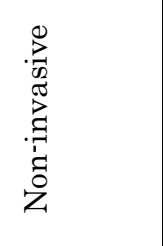 & 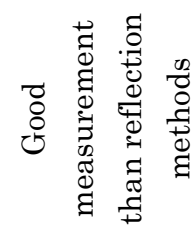 & 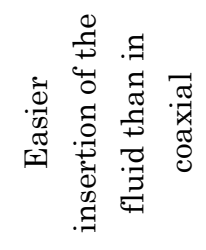 & 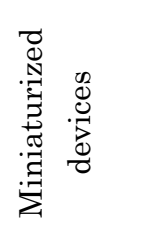 & 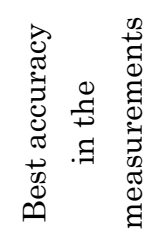 & 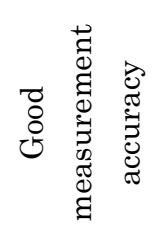 & 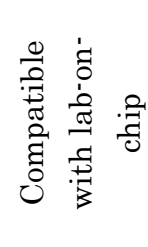 \\
\hline 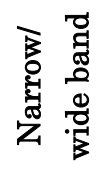 & $\overbrace{3}^{\circ}$ & 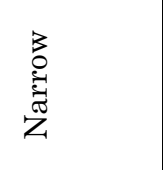 & $\overbrace{0}^{0}$ & 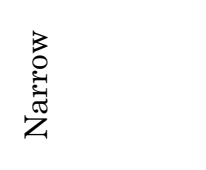 & $\overbrace{3}^{8}$ & 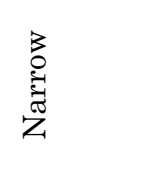 & 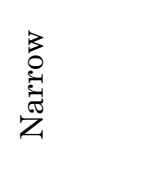 & 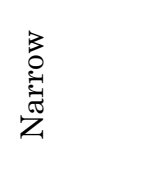 \\
\hline 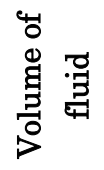 & 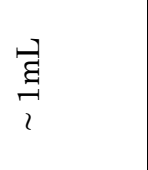 & 离 & $\begin{array}{l}\vec{g} \\
\mathrm{v}\end{array}$ & 咅 & 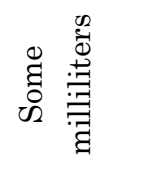 & ह & $\frac{\xi}{2}$ & 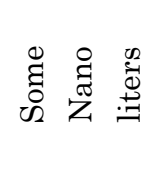 \\
\hline 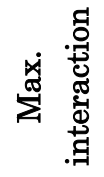 & $x$ & $x$ & $x$ & $>$ & $x$ & $>$ & $x$ & $x$ \\
\hline 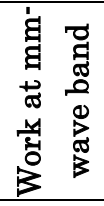 & 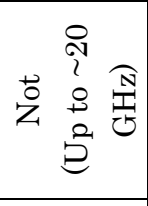 & 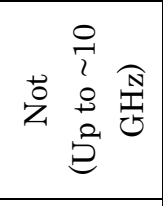 & 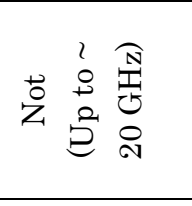 & 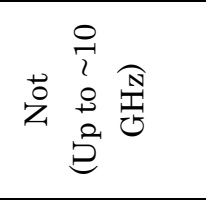 & 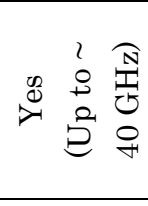 & 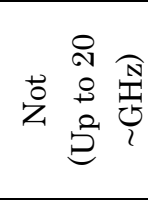 & 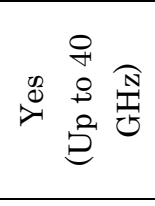 & 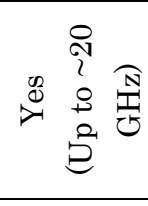 \\
\hline 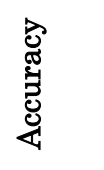 & (3) & 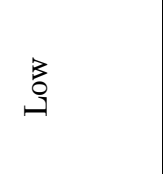 & $\begin{array}{l}8 \\
8 \\
\end{array}$ & : & $\begin{array}{l}\text { Z } \\
8 \\
0\end{array}$ & $\begin{array}{l}\overrightarrow{8} \\
8 \\
\infty \\
0 \\
0 \\
>0\end{array}$ & 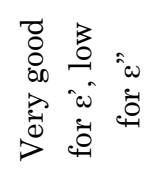 & $\begin{array}{l}8 \\
8 \\
0\end{array}$ \\
\hline 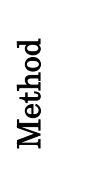 & 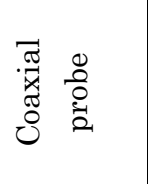 & 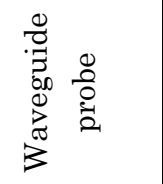 & 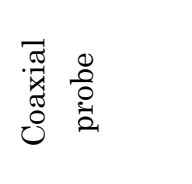 & 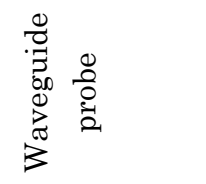 & 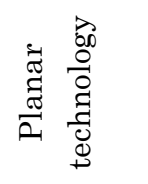 & 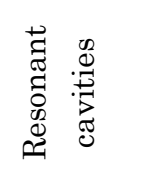 & 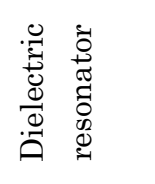 & 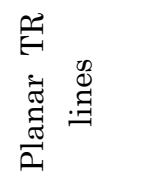 \\
\hline & \multicolumn{2}{|c|}{ 䆖 } & \multicolumn{3}{|c|}{ 总 } & \multicolumn{3}{|c|}{ थ્ર } \\
\hline
\end{tabular}




\subsection{Guiding structures in the millimetre-wave band}

The guiding structure must fulfil the following conditions:

- Work in the mm-wave band.

- Maximum concentration of the electromagnetic field in the desired interaction zone to improve the sensitivity.

- Technology suitable to create cavities and channels inside of the structure for microfluidic applications.

In sensor applications, rectangular waveguides or microstrip lines are used as a transmission media. However, the implementation of passive components and interconnected transmission lines are difficult at $\mathrm{mm}^{-}$waves due to significant losses. Therefore, they are typically packaged within an enclosure, which leads to the appearance of cavity modes that affect the overall performance. Hence, there is a need to develop new multi-functional highly-performing technologies for these bands. First, a review of the classical technologies in microwave applications is done.

\subsubsection{Metal waveguides}

Classical metal waveguides-based passive components can be very low loss even at microwave range spectrum and they are very suitable for low loss applications. However, traditional machining techniques for the fabrication of metal waveguides that operate at high frequency are complicated and costly [23], expensive post fabrication modification and mechanical assembly. Manufactured components in two blocks increase the difficulty to achieve the low loss and high $\mathrm{Q}$ values at high frequencies.

In addition, for fluid characterization, the main difficulty is how to hold the liquid inside the waveguide.

\subsubsection{Printed planar transmission lines}

Microstrip and coplanar lines (CPW) are the most representative planar transmission lines. They are robust, low cost and very suitable for integrating active microwave components on circuit boards. Nevertheless, the transmission properties of both (microstrip and CPW lines) greatly depend on the substrate parameters. In the $\mathrm{mm}^{-w}$ wave frequency range, these lines can present high 
insertion loss due to the presence of lossy dielectric material and that the E-field is not completely confined.

It is expected that high-density integration techniques, combined with a lowcost fabrication process, should be able to offer widespread solutions for future high frequency microwave or mm-wave commercial applications [24]. Researchers have come out with technologies such as Substrate Integrated Waveguide (SIW), low loss thin-film microstrip lines each of the proposed techniques has their own merits and demerits.

\subsubsection{Substrate Integrated Waveguide (SIW)}

Substrate Integrated Waveguide, also called post-wall waveguide or laminated waveguide, is a promising candidate for millimetre-wave applications. SIWs are integrated waveguide-like structures fabricated by using two rows of conducting cylinders or slots embedded in a substrate that electrically connect two parallel metal plates [25]. These structures exhibit propagation characteristics similar to those of rectangular metallic waveguides, provided that the metallic vias are closely spaced.

SIW devices can largely preserve the well-known advantages of conventional rectangular waveguides, namely high $\mathrm{Q}$ and high power capacity [26], and also it takes the advantages of microstrip lines, such as low profile, small volume and light weight. A priori, these advantages make it very suitable for the design of mm-wave circuits. SIW structures are subject to three mechanisms of losses: conductor, dielectric and radiation loss. This last one, it is a consequence of the periodic nature of the vias forming the sidewalls and if the design considerations are not assessed, leakage losses become a very important factor. Moreover, in these devices, propagation takes place through the dielectric, therefore increasing propagation losses. In Chapter 3, these limitations will be explained with more detail.

\subsubsection{Gap waveguide}

Recently, a new waveguide technology based on artificial surfaces for millimetre and sub-millimetre waves has been introduced [27]. This new guiding structure is created in the gap between two parallel metal plates. One of these plates has a textured surface made by means of a periodic pattern which acts as an Artificial Magnetic Conductor (AMC) when the distance between this pattern and the 
upper metal plate is less than $\lambda 0 / 4$. An AMC is nearly lossless reactive surface that presents a reflection coefficient of the order of 1 to incident waves and simultaneously inhibits the propagation of surface waves across its surface over a certain band of frequencies. Consequently, it produces a high impedance condition over a certain frequency range, emulating the properties of theoretical Perfect Magnetic Conductor (PMC). This means that the textured surface forbids the propagation of normal parallel plate modes in any direction as long as the distance to the opposing smooth plate is less than a quarter of wavelength. Still, local waves are allowed to propagate along metal ridge, strips or grooves present in the textured plate (Figure 1.24).

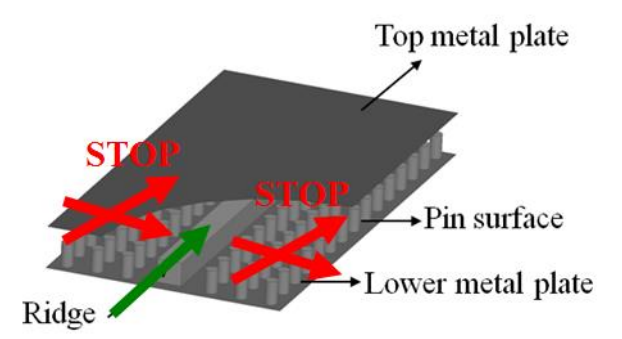

a) $3 \mathrm{D}$ view of the gap waveguide structure

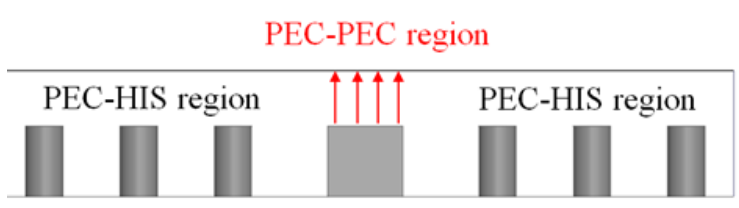

b) Front view of the gap waveguide

Figure 1.24. Gap waveguide operating principle.

Gap waveguide technology was developed as an attempt to overcome mechanical limitations in terms of metallic contact of the classical guiding structures. The general properties, basic theory and design of the gap waveguide are explained in Chapter 2.

\subsubsection{Conclusion}

Once, all the classical guiding structures have been analysed, we present the characteristics of each guiding structure according to our objectives in Table 1.2. We can conclude that gap waveguide is a good technology to work in the $\mathrm{mm}^{-}$ wave band and shows a high interest for microfluidic applications. This technology is used to create resonant cavities, which are one of the best techniques for fluid characterization, as mentioned above. Moreover, in gap waveguide the interaction with the fluid is done in the maximum E-field concentration improving the sensitivity of the sensor. 
Table 1.2. Properties of each guiding structures according to our objectives.

\begin{tabular}{|c|cccc|}
\hline & $\begin{array}{c}\text { Work in the } \\
\text { mm-wave } \\
\text { band }\end{array}$ & $\begin{array}{c}\text { Low } \\
\text { RF } \\
\text { access }\end{array}$ & $\begin{array}{c}\text { Compatible with } \\
\text { microfluidic } \\
\text { applications }\end{array}$ & $\begin{array}{c}\text { Maximum interaction } \\
\text { between the fluid and } \\
\text { the E-field }\end{array}$ \\
\hline Metal waveguide & - & +++ & - & - \\
\hline $\begin{array}{c}\text { Printed planar } \\
\text { transmission lines }\end{array}$ & ++ & + & ++ & - \\
\hline SIW & +++ & + & ++ & - \\
\hline Gap waveguide & +++ & ++ & +++ & $\checkmark$ \\
\hline
\end{tabular}

\subsection{Manufacturing techniques for microfluidic sensors}

Based on the choice of the planar technology for microfluidic sensors, we present in this section several manufacturing techniques for fluid characterization. These technologies are:

- $\mathrm{PCB}$

- Glass, silicon

- Polymers, PDMS

- LTCC

We are focused on those manufacturing techniques compatible with the gap waveguide technology.

\subsubsection{PCB}

In recent years, the development of PCB-based microfluidics containing pumps, electrodes and sensors for fluidic applications has been presented [28].

PCB technology can produce microfluidic devices for lab-on-a-chip applications, due to the high degree of integration. Typical methods to integrate PCB with microfluidics are based on the use of polymer materials.

In the PCB approach, a low cost process is used to fabricate components commercially available. These advantages allow us easy integration and enable a modular design approach. However, one of the principal issues of the PCB technology for microfluidic applications is the biocompatibility with the fluids 
under test. An example for fabricating microfluidic devices by using PCB is illustrated in Figure 1.25 [29].

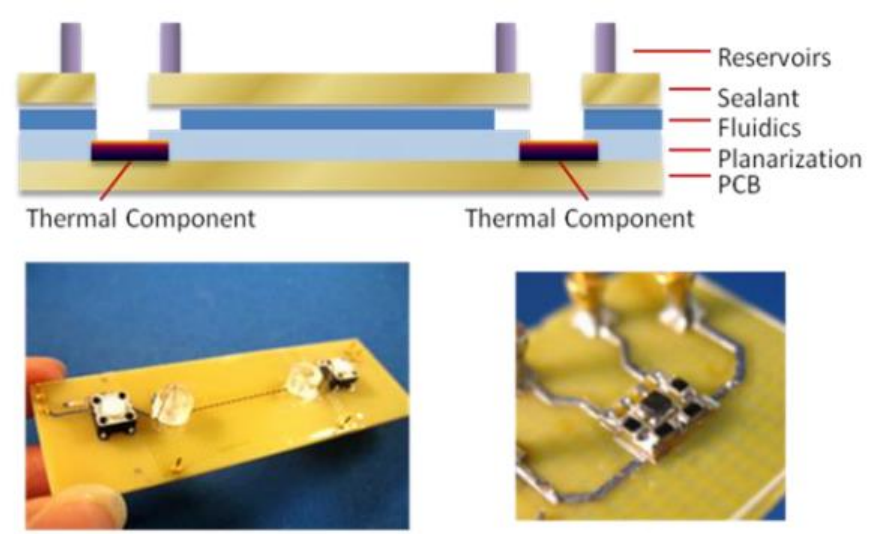

Figure 1.25. Microfluidic sensor based on PCB technology presented in [29].

\subsubsection{Silicon}

Historically, silicon microfabrication using MEMS-based processing techniques has been used for prototyping microfluidic devices [30] [31]. Silicon, however, has inherent drawbacks such as expensive clean room usage, impermeability to gases and optically opaque to certain electromagnetic wavelengths, limiting its applications in optical detection. To combat these shortcomings, glass and polymers appeared as a good solution to fabricate microfluidic devices.

\subsubsection{Polymers, PDMS}

As it is well known, for microfluidic applications one much extended technique is the utilization of polydimethylsiloxane (PDMS).

Whitesides et al. [32] demonstrated the use of PDMS for the fabrication of microfluidic structures using soft lithography technique. Polymers such as PDMS, PMMA, SU-8, Teflon, etc., have characteristics such as transparency, ease of surface modifications, low electrical and thermal conductivity, biocompatibility, and flexible substrates that have made them attractive materials for demonstration of lab-on-chip applications.

However, while polymers are relatively inexpensive materials, only a handful of them are processable and cost of lithography-based fabrication is significantly high. Moreover, the most important issues of the PDMS for the gap waveguide manufacturing are: 
- Metallization, creation and metal via filling

- High $\tan \delta$ value.

\subsubsection{LTCC technology}

One possible solution to overcome these limitations is Low Temperature Co-fired Ceramic (LTCC) technology which is especially useful for microwave applications because ceramic substrates and gold or silver conductive layers exhibit excellent physical properties [33]. Moreover, LTCC has been presented as biocompatible and suitable for microfluidic and lab-on-chip applications utilizing live cell sensing [34].

The term LTCC means that the ceramic dielectric structure, conductive, resistive, and other dielectric materials are fired at the same time at a relative low temperature (less than $900^{\circ} \mathrm{C}$ ). This technology has attained a key position in microelectronics, microsystems, and sensors integration [35].

LTCC was conceived as a technology that could have the advantages of both thick film and HTCC technologies. LTCC is a technology with excellent properties for packaging and MCM applications, rendering good conductors, low associated capacitances, simpler processes and high layer count. There are also, limitations in LTCC technology, such as tolerance of thick film passive components, minimal width of the films, shrinkage variations and poor thermal conductivity.

The fabrication process of LTCC systems is simple and fast. Cost of investment is much lower than in silicon or thin film industry. The advantages of LTCC are: good electrical and mechanical parameters, high reliability and stability, possibility of making three dimensional (3D) microstructures with cavities and channels [36], high level of integration (sensors, actuators, heating, cooling, microfluidic, electronic and photonic systems), very good properties at high voltage, high pressure and high vacuum. LTCC process is presented in Appendix A.

A great advantage of the LTCC technology is the low temperature of co-firing. It enables to use thick film materials [37] as well as various electronic components, sensors, actuators, microsystems, microreactors, cooling, heating systems and cavities and channels in one package as it is shown in Figure 4.1. 


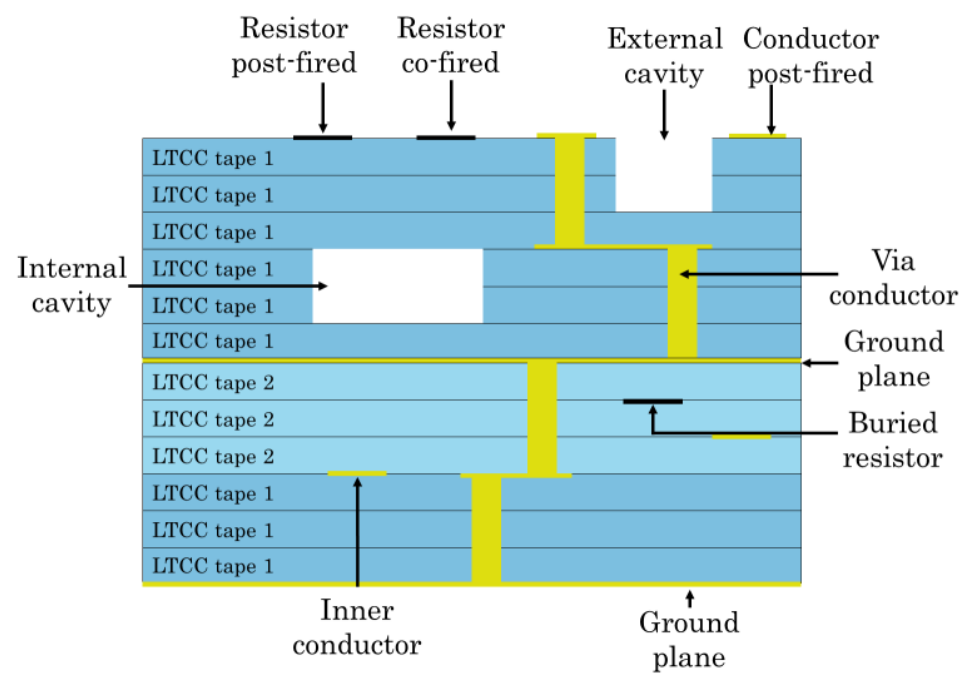

Figure 4.1. LTCC module example.

\subsubsection{Conclusion}

The summarized properties of each manufacturing technique according to our objectives are illustrated in Table 1.3. As we can see, LTCC appears as a very attractive technology for microfluidic applications due its biocompatibility. Moreover, LTCC 3D structures allow manufacturing cavities and channels and are capable to integrate RF passive components; being a good candidate for the gap waveguide manufacturing.

Table 1.3. Manufacturing techniques properties according to our objectives.

\begin{tabular}{|c|c|c|c|c|c|}
\hline & $\begin{array}{c}\text { Creation } \\
\text { of metallic } \\
\text { vias }\end{array}$ & $\begin{array}{l}\text { Serigraphy and } \\
\text { metallization of } \\
\text { transmission lines }\end{array}$ & $\begin{array}{c}\text { Cavities } \\
\text { and } \\
\text { channels }\end{array}$ & Biocompatible & Cost \\
\hline PCB & +++ & +++ & ++ & - & + \\
\hline Silicon & ++ & ++ & +++ & ++ & +++ \\
\hline $\begin{array}{c}\text { Polymers } \\
\text { (PDMS) }\end{array}$ & - & - & +++ & +++ & + \\
\hline LTCC & ++ & ++ & +++ & +++ & ++ \\
\hline
\end{tabular}




\subsection{Conclusion}

In this chapter we presented the context and the aim of the thesis. Our general purpose is to determine a structure acting as a sensor to characterize the electrical properties of fluids in the millimeter-wave band. With this purpose, firstly, the interest to increasing in frequency (up to $40 \mathrm{GHz}$ ) for fluid characterization is explained. The millimeter-wave band is presented as a good candidate to improve the sensitivity in the fluid measurement.

Next, a state of the art of the measurement methods for fluid characterization at the microwave range has been done. The studied literature shows that in terms of accuracy, the best solution is based on resonant cavities. However, their volume limits the applications being non-compatible with lab-on-chip techniques.

Once the operating frequency band has been chosen, we focused on the choice of the structure capable to work in the $\mathrm{mm}^{-}$wave band with low losses and compatible with measurement methods of fluid characterization. In this context, a new technology called gap waveguide presents good properties for both requirements. In this structure the wave propagation is done in the air cavity, so we can make resonators based in this technology. Moreover, given its topology, gap waveguides offer the possibility to integrate cavities and channels inside of them.

Once the measurement method, the operating frequency band and the topology have been selected, we concentrated in the technology for its implementation. Literature review shows that the classical methods for manufacturing microfluidic devices are: PCB, silicon, glass, polymers (PDMS) and LTCC. We have concluded that PCB and LTCC are possible techniques for the gap waveguide manufacturing. PCB presents good performances in terms of via metallization and low cost. However, this material is not very biocompatible for implant applications. In this sense, ceramic processes as LTCC is more suitable in terms of biocompatibility. Moreover, LTCC is very appropriate for the gap waveguide manufacturing. Metallization of vias in PDMS and its high tan $\delta$ value are drawbacks for the gap waveguide implementation. However, the metallization of vias and the creation of cavities in one co-fired process allow us the fabrication of the gap waveguide with LTCC including the fluidic channel in one step. 


\section{Chapter 2}

\section{Gap Waveguide Technology- Current Status and Contributions}

\subsection{Introduction}

In the millimetre-wave band, the implementation of passive components and interconnected transmission lines are critical, mainly due to the small size devices and significant losses. Manufacturing techniques of $\mathrm{mm}$-wave devices should ensure the possibility to integrate circuits which are small in size, compact and offer low transmission loss. Hence, there is a need to develop new multi-functional highly-performing technologies for these bands.

As it has been introduced in Chapter 1, gap waveguide can overcome most of the principal issues of the guiding structures in the millimeter-wave band. In this chapter, we propose an exhaustive study about the gap waveguide design and the most important electromagnetic properties of this topology.

In this context, this chapter is organized as follows: firstly, an overview of the gap waveguide technology is presented. In this section, the basic theory and the 
operating principle of the gap waveguide is revised. Then, the gap waveguide design using dielectric substrate compatible with PCB (Print Circuit Board) or LTCC technology is shown. On this particular implementation, the influence of some parameters on the frequency range of the stop-band zone has been studied. An analysis method for the characteristic impedance of gap waveguide using dielectrics to support the pin surface has been developed as well. Finally, next section shows a study of global losses of the microstrip gap waveguide where some design rules to improve the $\mathrm{Q}$-factor are proposed.

\subsection{Overview of the gap waveguide technology}

\subsubsection{Basic theory of the gap waveguide}

The gap waveguide is a new waveguiding topology recently presented [38]. It is based on the concept of artificial magnetic conductor surfaces (AMC) or metamaterial. Different meanings for the term metamaterial have been introduced in the literature, such as: left-handed (LH) materials, double negative (DNG) materials, negative refractive index (NRI) materials, magneto dielectric material, Soft and Hard surfaces, high impedance surfaces (HIS) and artificial magnetic conductor (AMC). In a conventional parallel-plate waveguide, when one of the plates is provided with a textured surface by a periodic surface of pins, approximately $\lambda / 4$ in height, it creates a high-impedance condition so that parallel-plate modes are forbidden, provided the gap between the nails top and the upper plate is smaller than $\lambda / 4$. The origin of the gap waveguide is the concept of soft and hard surfaces described in [39]. In the electromagnetic domain, a soft surface is a surface along which the power density flux (Poynting vector) is zero for any polarization. This means, that any electromagnetic wave will propagate along a soft surface. The behavior of the soft surface is a perfect electric conductor (PEC) for TE polarization and as a perfect magnetic conductor (PMC) for TM polarization, (vice-versa for the hard surface). These surfaces are in relationship with the EBG (Electromagnetic Band-Gap) surfaces as they exhibit a bandgap or stop-band for a certain range of frequencies where there is no field propagation. The stop-band in soft or hard surfaces appears only for a given direction; therefore it can be seen like the 1-D equivalent of an EBG surface. Figure 2.1 shows the propagation in a hard waveguide. In Figure 2.2 and Figure 2.3, the principle of operation of the gap waveguide is represented. The PMC (Perfect Magnetic Conductor) condition is the ideally AMC condition. 
Finally, gap waveguide can be considered as a half strip-line as it is represented in Figure 2.4.

The principle of operation of the gap waveguide is demonstrated in [40] where analytical solutions and dispersion characteristics are described. The pins work as a high-impedance surface (HIS) creating equivalent PMC condition, within a stop-band condition defined by a lower and upper cut-off frequency.

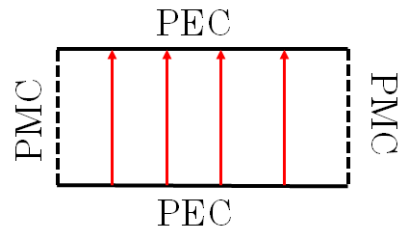

Figure 2.1 Linearly polarized hard waveguide.

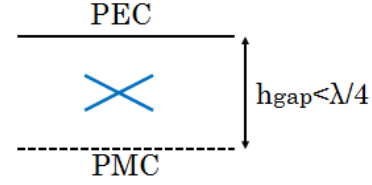

Figure 2.2 No propagation between PEC and PMC condition when $\mathrm{h}<\mathrm{\lambda} / 4$.

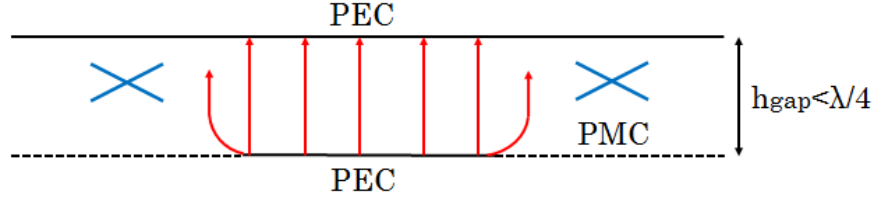

Figure 2.3. Ideal gap waveguide.

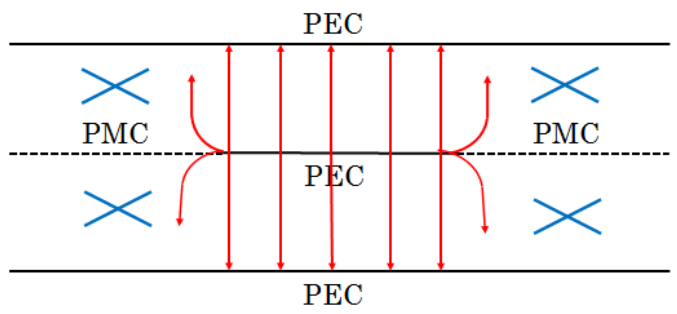

Figure 2.4. Stripline configuration.

The lower cut-off frequency is defined by the height of the pins being approximately $\mathrm{h}=\lambda / 4$, with $\lambda=\lambda_{0} / \sqrt{\varepsilon_{r}}$ to transform a short circuit (PEC) to an open circuit (PMC). Considering hgap as the height of the air gap, hgap should be less than $\lambda_{0} / 4$. This cut-off frequency means that for certain frequency bands and modes, waves become evanescent and thus the structure exhibits stop-band characteristics. The basic configuration of the ridge gap waveguide is shown in Figure 2.5. In Figure 2.6, the front view of the structure with the propagated field confined between the ridge and the upper metal plate is sketched. The stop-band is created by the pin surface that establish the HIS condition. 


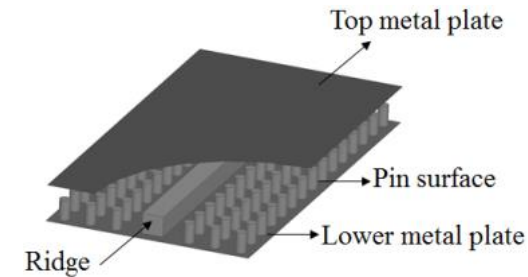

Figure 2.5. Geometry of the ridge gap waveguide.

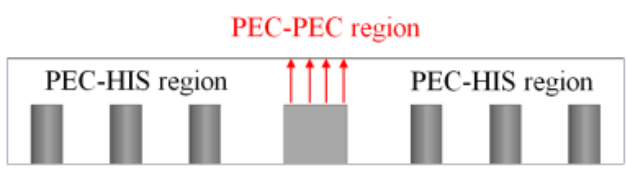

Figure 2.6. Front view of the ridge gap waveguide.

\subsubsection{Classification of the structures based on the gap waveguide concept}

Gap waveguide exists in three kinds of guiding structures: ridge gap waveguide, groove gap waveguide and microstrip gap waveguide [41].

\section{Ridge gap waveguide}

The ridge gap waveguide (Figure 2.7) is made with two parallel plates, an upper metal smooth lid and a lower plate, provided of a central metal ridge surrounded by the bed of nails. The bed of nails creates the high impedance surface which stops all parallel-plate modes. The field is confined between the ridge and the upper metal plate, where a quasi-TEM mode is propagated along the ridge [42].

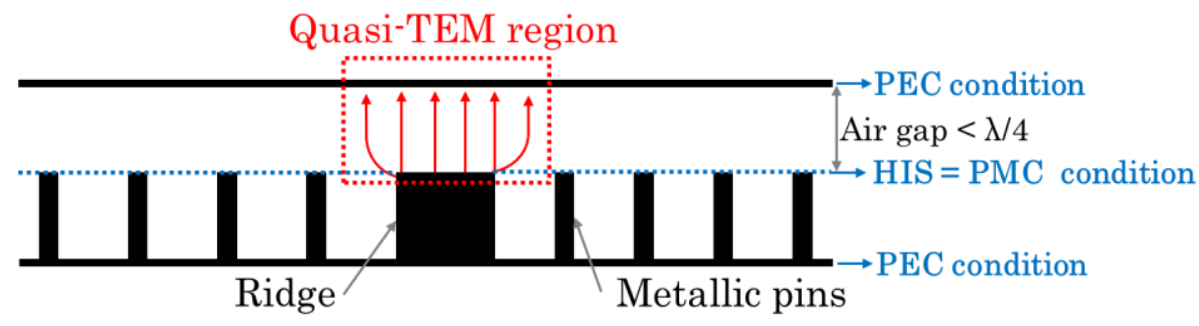

Figure 2.7. Ridge gap waveguide configuration.

\section{Groove gap waveguide}

Groove gap waveguide is an alternate of rectangular hollow waveguide. In the groove gap waveguide, propagation takes place on a groove embedded by the pins on both sides (Figure 2.8). The difference with the ridge gap waveguide is that the groove case supports a mode very similar to TE10 mode of rectangular waveguide. The advantages of the groove gap waveguide over a rectangular waveguide are the benefit of having contactless metal plates. Groove gap waveguide has less loss 
than ridge case because there is more volume for the current and the ridge is removed [43].

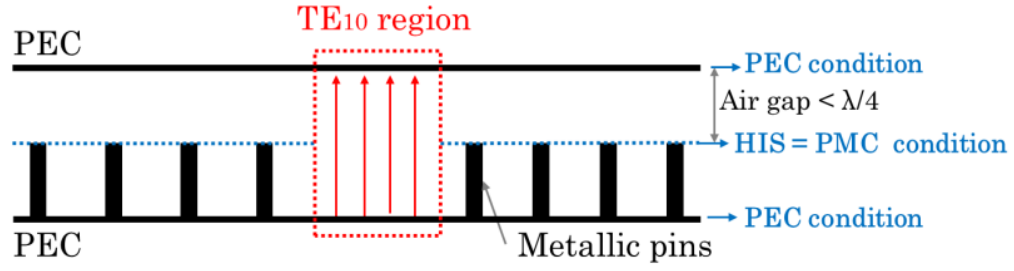

Figure 2.8. Groove gap waveguide - Vertical polarization.

\section{Microstrip gap waveguide}

The microstrip gap waveguide works similarly to inverted/suspended microstrip lines with the difference that the substrate is supported by the artificial magnetic conductor. Gap waveguide using a suspended strip is presented in [44]. It has been demonstrated three geometries based on the microstrip gap waveguide concept. In the three cases, the quasi-TEM mode is propagated in the air between the strip and the upper metal plate (Figure 2.9-Figure 2.11).

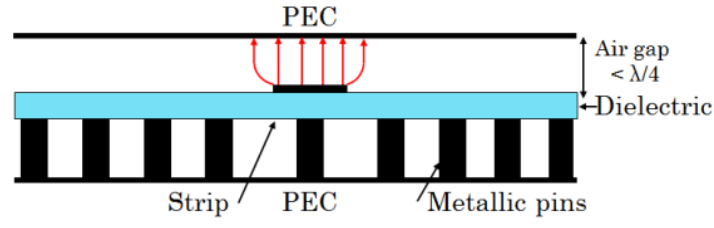

Figure 2.9. HIS is made with a surface of pins.

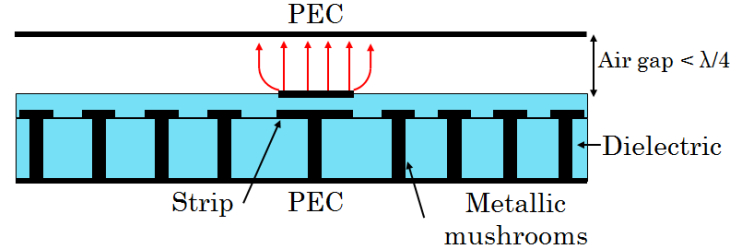

Figure 2.10. HIS made with mushroom shape surface embedded in dielectric.

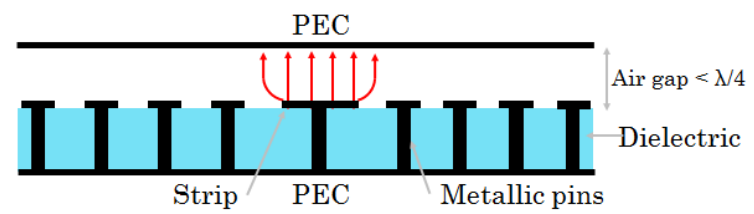

Figure 2.11. Mushroom surface and strip share the same substrate.

\subsubsection{Principle of operation of the gap waveguide}

Gap waveguide is based on the concept of artificial material conductor surfaces. These surfaces have the main property to stop the propagation in all directions. One approach to obtain the computed stop-band frequencies is the eigenmode 
analysis with a full-wave EM solver. In this thesis, we use HFSS solver because it includes an eigenmode calculation tool for a unit cell with periodic boundaries. Several shapes, such as metallic pins or mushrooms allow the creation of a surface with high impedance properties.

To illustrate the computation process, we use a mushroom surface based on Sievenpiper's [45] concept. In Figure 2.12, the cross-section of a high impedance surface created by a periodic mushroom shape surface is represented. As it is seen in Figure 2.13, due to the proximity of the top metal patches of the mushrooms, a capacitance effect is created, while the inductance is originated of the current loops within the structure.

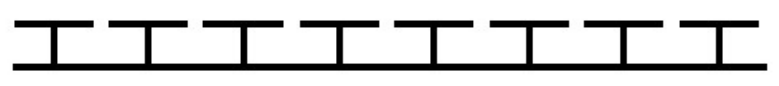

Figure 2.12. Cross-section of a periodic pattern of metallic mushrooms.

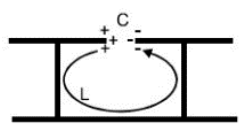

Figure 2.13. Capacitance and inductance of the circuit model.

In the Figure 2.15, the Brillouin zone diagram of the periodic pattern of mushrooms described in Figure 2.14 is represented. The first Brillouin zone can be reduced to a smaller region denoted by $\Gamma, \mathrm{X}$, and $\mathrm{M}$, corresponding to $(\mathrm{kx}=0$, $\mathrm{ky}=0), \quad(\mathrm{kx}=\Pi / \mathrm{d}, \mathrm{ky}=0)$, and $(\mathrm{kx}=\Pi / \mathrm{d}, \mathrm{ky}=\Pi / \mathrm{d})$, respectively. The irreducible Brillouin zone is the triangular wedge, which is one-eighth of the area of the full Brillouin zone, surrounded by three points: $\Gamma$, X, and M. Figure 2.16 shows the dispersion diagram created by one cell of the periodic structure. Axe $\mathrm{x}$ represents the boundary of the triangular wedge starting from $\Gamma$ through $\mathrm{X}$ and $\mathrm{M}$ and returning to $\Gamma$. The three bands correspond to different incident conditions: (1) from $\Gamma$ to $\mathrm{X}$, the $\mathrm{x}$-component of phase constant $\mathrm{kx}$ is increasing from zero to $\mathrm{n} / \mathrm{d}$ while the $\mathrm{y}^{-c o m p o n e n t}$ of phase constant ky is set to zero; (2) from $\mathrm{X}$ to $\mathrm{M}$, ky is increasing from zero to $\Pi / d$ at a fixed $x^{-}$component of phase constant $k x=\Pi / d$, (3) from $\mathrm{M}$ to $\Gamma$, we keep $\mathrm{kx}=\mathrm{ky}$ and iterate it from $\mathrm{n} / \mathrm{d}$ to zero along the diagonal segment. 


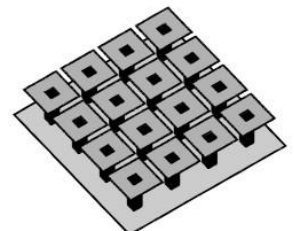

Figure 2.14. Periodic mushroom shape pattern.
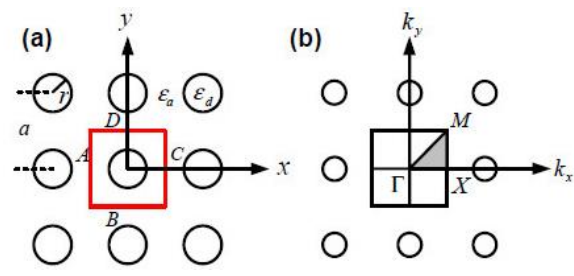

Figure 2.15. Brillouin zone of periodic pattern.

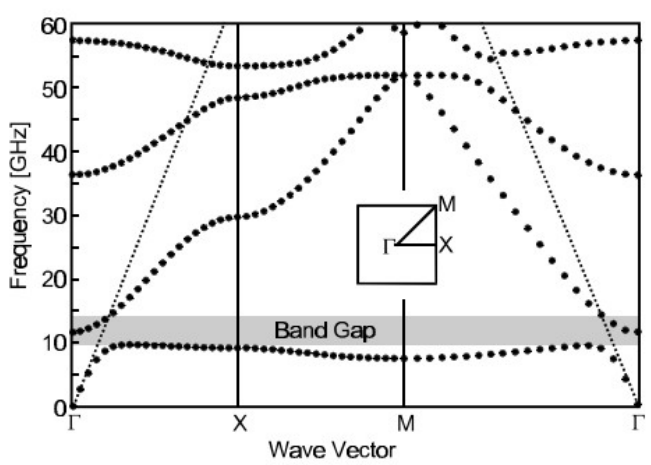

Figure 2.16. Dispersion diagram of the mushroom surface.

In this section, the procedure to calculate the dispersion diagram of the periodic pattern of cells has been described. In this thesis, the upcoming analysis of the dispersion diagram will be based on this procedure.

\subsection{Design of the microstrip gap waveguide compatible with PCB or LTCC technology}

The high impedance surface can be created by a pin or mushroom surface. In Figure 2.17, the boundary conditions of the pin unit cell are presented. Originally gap waveguide do not need the use of dielectric; nevertheless, in this study, it is considered pin or mushroom surface embedded in dielectric to be compatible with PCB technology or LTCC technology. It has been chosen PEC conditions for the metallic pin and the LTCC tape ESL41110 with permittivity $\varepsilon_{\mathrm{r}}=4.2$ and dielectric loss tangent $\tan \delta=0.004$ (values proportioned by the manufacturer) is selected as a dielectric material. Pin height is $675 \mu \mathrm{m}$, the air gap height is $450 \mu \mathrm{m}$, the diameter of the pin is $200 \mu \mathrm{m}$ and the distance between pins is $600 \mu \mathrm{m}$.

Master and slave boundaries allow to model infinitely large repeating array structures. The E-field on one surface, the slave surface is forced to match the E- 
field on another, the master surface, to within a phase difference. The boundary conditions defined by the master and slave boundary of the unit cell are represented in Figure 2.17-b and in Figure 2.17-c, respectively.

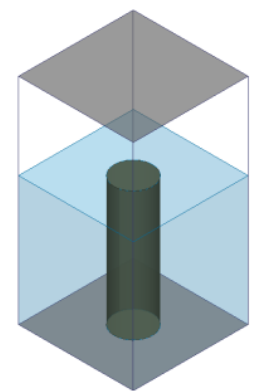

a)

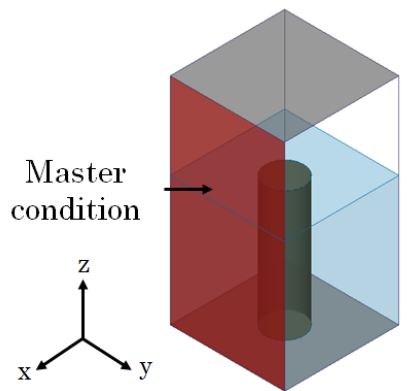

b)

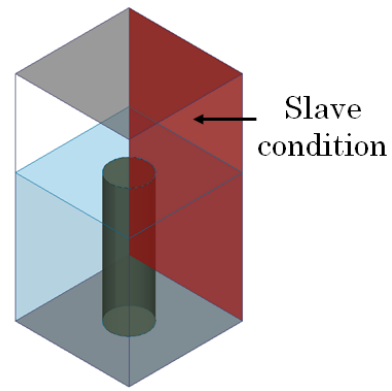

c)

Figure 2.17. Boundary definition to calculate dispersion diagram of periodic pins: a) Pin unit cell, b) Master boundary condition, c) Slave boundary condition.

Using the finite element method, the metal and dielectric regions of one unit cell are discretized on grid. The electric field at all points on the discretized surface can be reduced to an eigenmode equation, which can be solved numerically. For this reason, with the Eigenmode of HFSS solver, we can calculate the dispersion diagram created by the surface. This diagram constitutes a graph of frequency versus the propagation constant, from which we can know what modes will or not will not be excited at a certain frequency. In Figure 2.18, the dispersion diagram created by the pin surface is illustrated. We can see the stop-band created between $51.3 \mathrm{GHz}$ and $69.1 \mathrm{GHz}$.

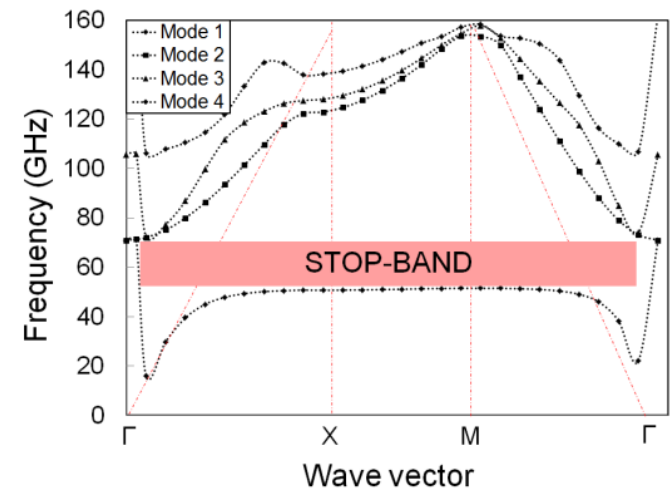

Figure 2.18. Dispersion diagram created by the pin surface. 
Considering the same dimensions described above and introducing a patch on the top of the pin with diameter of $300 \mu \mathrm{m}$, the dispersion diagram is represented in the Figure 2.19. The stop-band created by the mushroom surface is between [48.7 and 69.1] GHz. In the case of the gap waveguide designed with mushrooms, the diameter of the pads on the top of the via-hole pins also affects the stop-band. As it is explained in [46], by keeping the period and via-hole diameter fixed, an increase in the pad diameter will shift the stop-band toward lower frequencies.
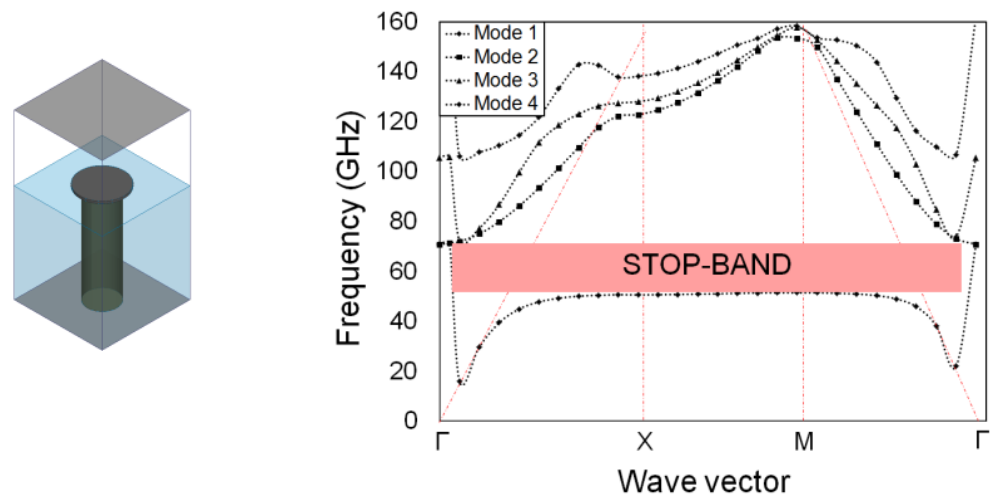

Figure 2.19. Dispersion diagram created by the mushroom surface.

In previous works [47], it is related that the pins surface is more suitable than mushroom surface at high frequency, due their physical implementation and volume. However, by changing some dimensions like the diameter of the patch, we can modify the minimum frequency of the stop-band; therefore we can increase the total bandwidth of the stop-band. Mushroom shape allows increasing the bandwidth of the stop-band, and also, to reduce dimensions in terms of pin height. For this, a mushroom surface is used in this work for the losses study and for the posterior fabrication.

Once, the stop-band created by the HIS is defined, next step consists on the analysis of the effect of a ridge crossing the bed of pins. The ridge will originate a quasi-TEM mode propagating in the stop-band. In Figure 2.20-a, the complete structure of the gap waveguide is represented. If we made a cut as it is shown in red in this figure, we obtain the Figure 2.20-b. This row of pins and ridge is used to obtain the dispersion diagram by the defined master and slave conditions in the front and back boundary conditions. 

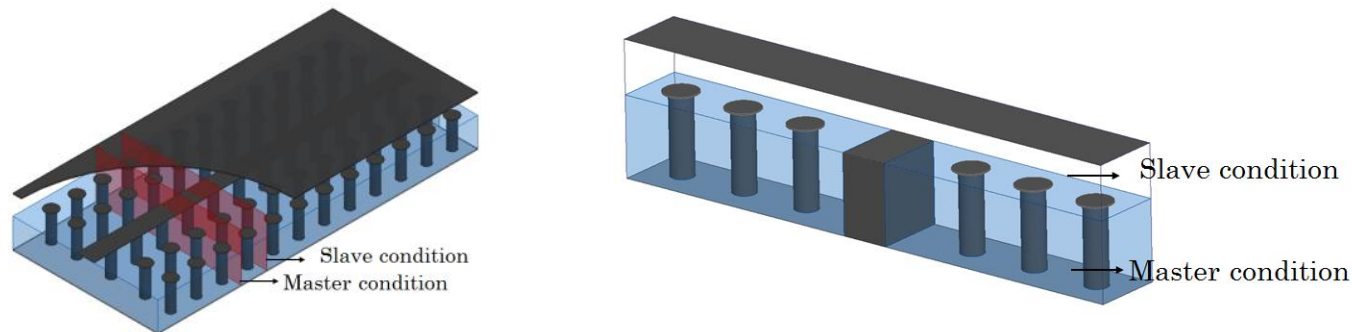

Figure 2.20. a) 3-D view of the gap waveguide structure. b) Example of the boundary conditions in one row of pins to obtain the dispersion diagram.

The dispersion diagram computed by Eigenmode solver of the design is depicted in Figure 2.21, and in Figure 2.22, the S-parameters graph is obtained with the Driven Model of HFSS solver. The stop-band corresponds with the stopband calculated by the surface of mushrooms described above. The upper limit of the stop-band can be identified as the frequency value at insertion loss increases.

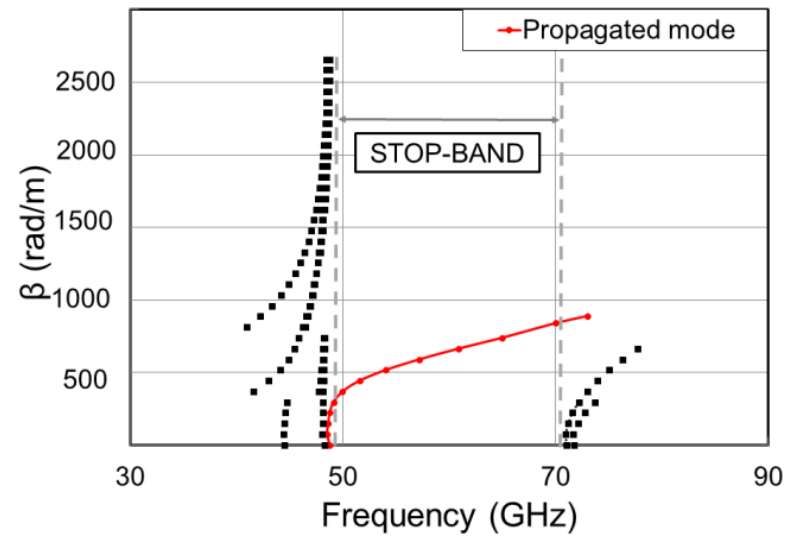

Figure 2.21. Dispersion diagram of one row of mushrooms and ridge.

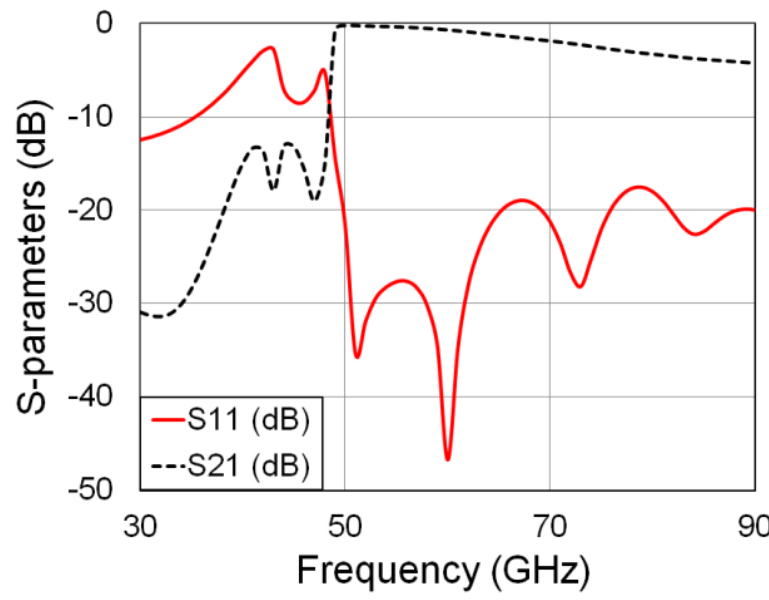

Figure 2.22. S-parameters of the gap waveguide with mushroom surface. 
We can observe a difference between the stop-band obtained by the dispersion diagram and the computed S-parameters. From $70 \mathrm{GHz}$, the stop-band does not exist, and the multi-modal behavior begins. For this reason, transmission parameter decreases due to the multi-modal propagation. The difference with the dispersion diagram response can be due to the fact that the dispersion diagram is calculated based on an infinite unit cell, when in reality we always design our geometries by using a finite periodic pattern.

In Table 2.1, the vertical E-field distribution at the surface of the upper metal plate of the gap waveguide for some meaningful frequencies is represented. We can see than outside the stop-band, E-field is dispersed along all the upper metal plate. Nevertheless, inside the range of frequencies of the stop-band, the E-field is confined between the ridge or strip and the upper metal plate.

Table 2.1. Vertical E-field distribution at the surface of the upper metal plate.

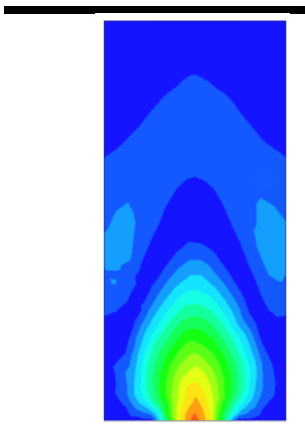

$35 \mathrm{GHz}$ (outside the stop-band)

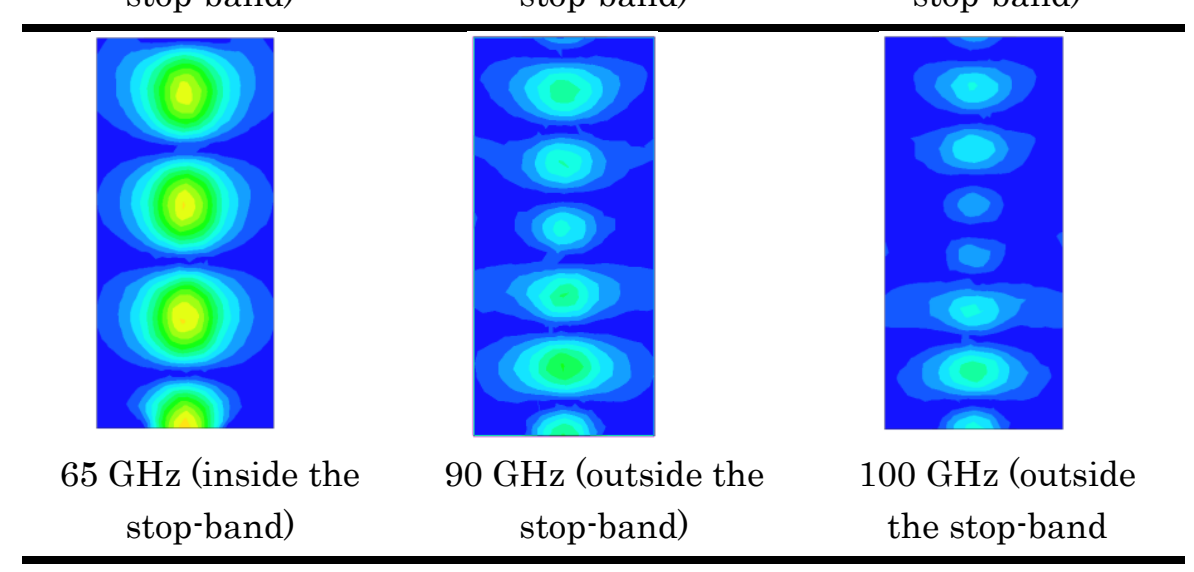

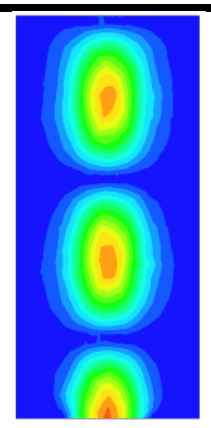

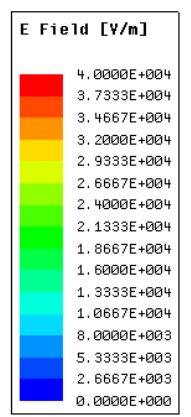

$45 \mathrm{GHz}$ (outside the

$55 \mathrm{GHz}$ (inside the

stop-band)

stop-band) stop-band)

the stop-band

The gap waveguide design presented in this section exhibits the expected behavior. Namely, in the stop-band region, only the quasi-TEM mode is 
propagated and outside this band, the E-field is dispersed through the entire air cavity.

\subsubsection{Stop-band study}

The stop-band created by the pin surface acting as a HIS condition is mostly defined by the pin height $(\mathrm{hp})$ and the condition hgap $<\lambda_{0} / 4$. However, other parameters can impact in the stop-band relationship such as the distance between pins or the diameter of them. In next part the impact in the stop-band of some parameters is analyzed.

\subsubsection{Air Gap height impact}

One of the critical parameters is the air gap height (hgap), which sets the upper limit of the stop-band. A study determining the cut-off frequencies as a function of the geometrical parameters of the periodic surface, including the pin surface, and the ridge width, has already been reported in [48]. In that work, also analytical formulas for the determination of the lower and upper cut-off frequencies of the stop-band have been obtained. However, these formulas are only valid asymptotically for a small period, for the gap waveguide technology without dielectric. We propose to complete the study in [11], applying it to the topology described in previous section: the gap waveguide with dielectric, and considering the same dimensions.

In [49] it is demonstrated that for small periods, the pin surface works as a HIS when they are approximately $\mathrm{hp}=\lambda / 4\left(\lambda=\lambda 0 / \sqrt{\varepsilon}_{\mathrm{r}}\right)$ high, and they will provide a stop-band from this frequency to a frequency at which $h p+h g a p=\lambda / 2$, where hgap is the height of the air gap.

So, fixing the pin height, the diameter of the pin, the diameter of the patch and varying only the gap height, the relationship between this height of the air cavity and the stop-band is shown in Figure 2.23. The maximum value of hgap is 1.25 $\mathrm{mm}$ that would correspond to $\lambda_{0} / 4$. Varying the gap height, the minimum frequency of the stop-band remains without variation, but the maximum frequency of the stop-band decreases as the gap height increases. We can confirm the same conclusion published in previous work, because the use of the dielectric covering the pin surface is mostly independent of the impact of the gap height over the stop-band. 


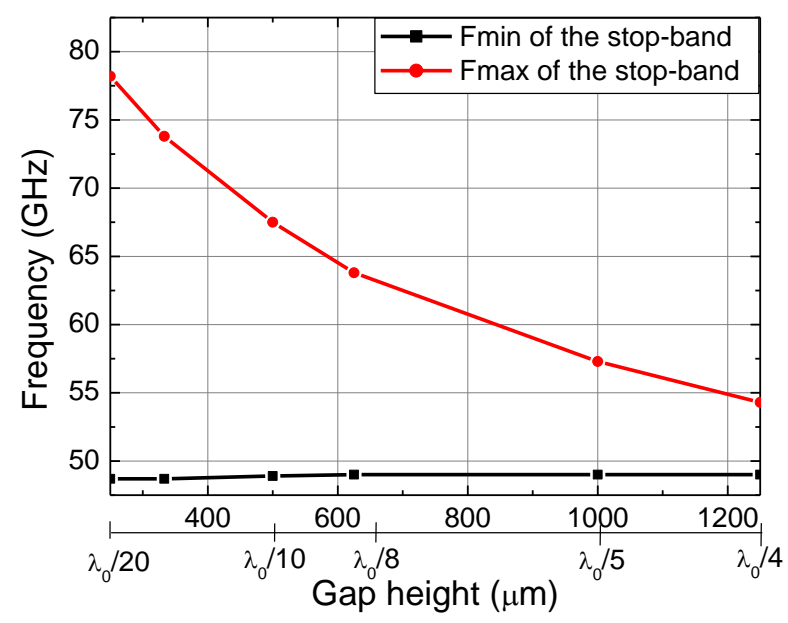

Figure 2.23. Minimum and maximum frequency of the stop-band by varying the gap height.

The stop-band bandwidth is shown in Figure 2.24. The lower the gap height, the wider the bandwidth of the stop-band is.

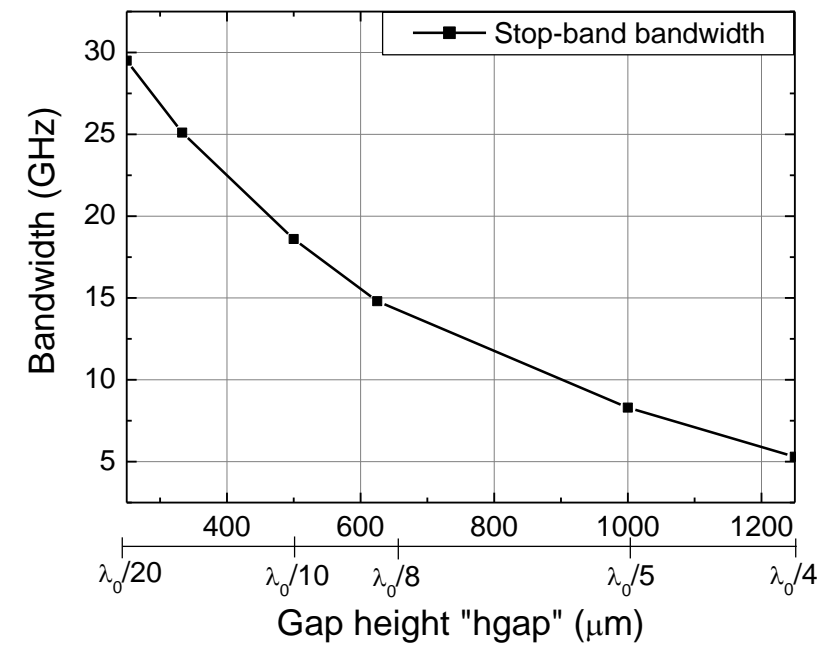

Figure 2.24. Gap height impact over the bandwidth of the stop-band.

\subsubsection{Influence of the distance between pins over the bandwidth of the stop-band}

Although the most important parameters that impact the stop-band are the pin height and the air gap height, other parameter may have an influence as well. In 
particular, we seek to optimize pin periodicity since it may become a key constraint during fabrication.

In the case of the mushroom type EBG surface, the minimum distance between pins has to be a little bit larger than in the case of original bed of nails, due to the diameter of the patch.

We fix gap height value at hgap $=450 \mathrm{um}\left(\sim \mathrm{\lambda}_{0} / 11\right.$ at $\left.\mathrm{f}_{0}=60 \mathrm{GHz}\right)$, pin diameter $=200 \mu \mathrm{m}$ and diameter of patch $=300 \mu \mathrm{m}$. Figure 2.25 shows the impact in the minimum and maximum frequency of the stop-band if we change the relationship between pin spacing $\mathrm{p}$ and pin diameter $\mathrm{d}$. Results show that the maximum frequency is mostly affected by the variation of the spacing between pins. If we increase this spacing, the maximum frequency increases, hence the bandwidth increases until the ratio $\mathrm{p} / \mathrm{d}=5$ is reached.

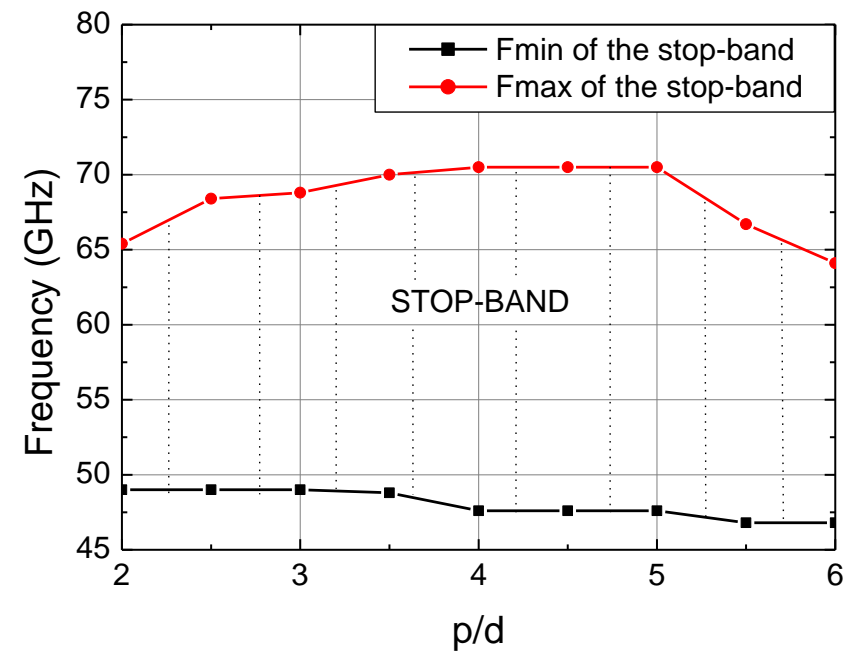

Figure 2.25. Impact of the ratio pin_periodicity/diameter over the minimum and maximum frequency of the stop-band with the mushroom surface.

Figure 2.26 shows the behavior of the stopband bandwidth as spacing between pins is varied. Between $\mathrm{p} / \mathrm{d}=2$ and $\mathrm{p} / \mathrm{d}=4$, an increase in bandwidth is observed. From this value, the bandwidth is constant and after it decreases. 


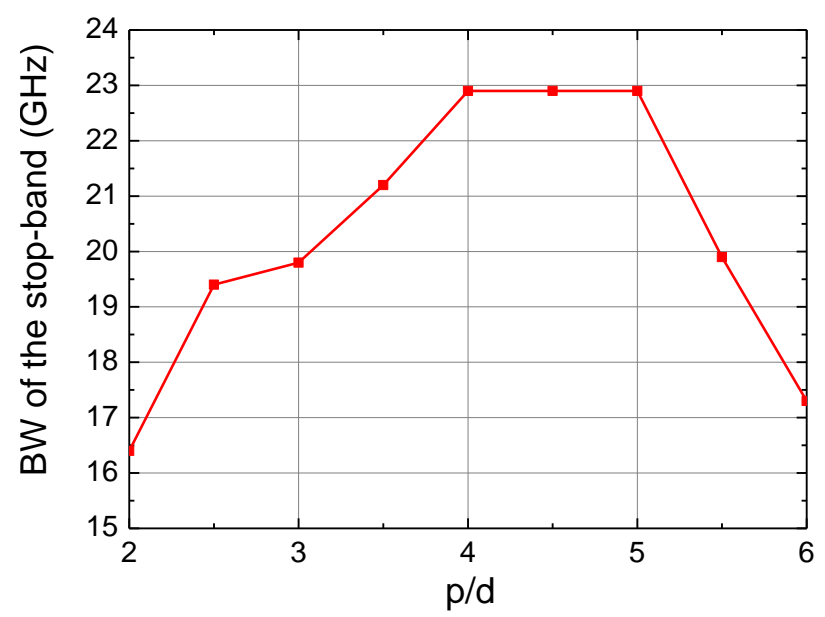

Figure 2.26. Impact of the ratio pin_periodicity/diameter over the BW of the stop-band.

As conclusion, the pin height and the air gap height are the most important contributions for the stop-band. Nevertheless, as it has been explained other parameters as the periodicity of the pins and the pin diameter can impact in the stop-band. In this sense, if the pin and air gap height are fixed, with the relationship p/d we can obtain a maximum bandwidth of the stop-band between $\mathrm{p} / \mathrm{d}=4$ and $\mathrm{p} / \mathrm{d}=5$.

\subsubsection{Impedance analysis methods}

To characterize a guiding structure, one of the most important parameters is the characteristic impedance. Initially, the characteristic impedance of the gap waveguide is given approximately by that of a microstrip line. As it is shown in Figure 2.27-a and Figure 2.27-b, the region where the propagation takes place in the gap waveguide is similar that an inverted microstrip line.

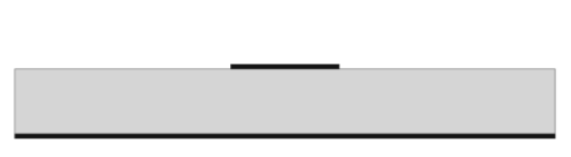

a)

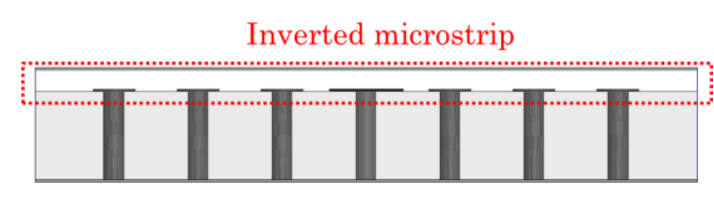

b)

Figure 2.27. Front view of: a) Microstrip line, b) Gap waveguide. 
In ref [50] a numerical study based on electromagnetic simulations is proposed for the determination of the characteristic impedance of the ideal gap waveguide (ridge surrounded by a bed of nails surface where no dielectric is needed). In [51] an analytical expression of the modal dispersion equation has been described of the first two modes and of the characteristic impedance of the dominant mode for a metal gap waveguide surrounded by a bed of nails.

As it is explained in the literature, in the case of the gap waveguide without dielectric, three approaches to calculate the impedance value had been demonstrated. In this section, with the same procedure described in [52], impedance methods for the microstrip gap waveguide with dielectric are analyzed.

The dimensions of the gap waveguide are those given at the beginning of this section and they are gathered in Table 2.2 for clarity.

Table 2.2. Gap waveguide parameters.

\begin{tabular}{|ccc|}
\hline \multicolumn{3}{|c|}{ Gap waveguide parameters } \\
\hline Pin diameter & $\mathrm{d}$ & $200 \mu \mathrm{m}$ \\
\hline Spacing of the pins & $\mathrm{p}$ & $600 \mu \mathrm{m}$ \\
\hline Dielectric & $\begin{array}{l}\text { LTCC tape: } \\
\text { Ferro A6-M }\end{array}$ & $\begin{array}{c}\varepsilon \mathrm{r}=4.2 \\
\text { Tan } \delta=0.004 \\
\text { Layer thickness }=75 \mu \mathrm{m}\end{array}$ \\
\hline $\begin{array}{c}\text { Pin height (or } \\
\text { substrate thickness) }\end{array}$ & $\mathrm{hp}$ & $675 \mu \mathrm{m}$ (9 LTCC layers) \\
\hline Gap height & hgap & $450 \mu \mathrm{m} \approx \lambda \mathrm{o} / 11$ \\
\hline Diameter of the patch & $\mathrm{d} 2$ & $300 \mu \mathrm{m}$ \\
\hline
\end{tabular}

We define three approaches for the characterization of the characteristic impedance of the gap waveguide with dielectric:

\section{1) First approach}

The configuration of the gap waveguide is PEC-PMC-PEC. If we apply image theory to the PMC plate, the gap waveguide can be seen as a "half" strip-line. For this reason, we can generalize that (2.1):

$$
\mathrm{Z}_{\text {gap waveguide }}=2 \mathrm{Z}_{\text {stripline }}
$$




\section{2) Second approach}

Using the electric and magnetic fields, we can calculate the characteristic impedance. If we consider an infinitely long transmission line, with an alternating current voltage $\mathrm{V}$ defined across the conductors, a longitudinal current I will be result. The characteristic impedance can be deducted from:

$$
\mathrm{Z}_{\mathrm{VI}}=\frac{\mathrm{V}}{\mathrm{I}}
$$

where,

$$
\mathrm{V}=\int \mathrm{E}_{\mathrm{y}} \mathrm{dy} \quad \mathrm{I}=\int \mathrm{H}_{\mathrm{x}} \mathrm{dx}
$$

$\mathrm{V}$ and I are calculated in a cross section of the line. $\mathrm{V}$ is the voltage obtained as the field integral of the vertical component of the electric field ( $\mathrm{E}_{\mathrm{y}}$ ) along a straight vertical line that links the center point of the ridge with the metal plate on top. The transverse current I is obtained by integrating the tangential magnetic field on the surface of the strip. In this case it is orientated in the $\mathrm{x}^{-}$ direction $(\mathrm{Hx})$ (Figure 2.28).

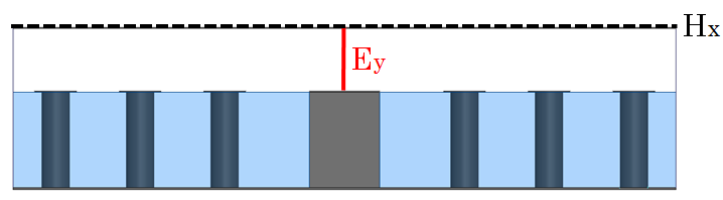

Figure 2.28. Integration lines used in the simulations

\section{3) Third approach}

This approach is based on the definition of the characteristic impedance as showed in (2.5):

$$
\mathrm{Z}_{\mathrm{PI}}=\frac{\mathrm{P}}{\mathrm{I}^{2}}
$$

Where $\mathrm{P}$ is obtained by integrating the $\mathrm{z}$ component (longitudinal) of the Poynting vector over a cross section (S), as defined in (2.6). The integration surface has been considered as it is shown in Figure 2.29.

$$
P=\iint_{S}\left(E_{x} H_{y}^{*}-E_{y} H_{x}^{*}\right) d S
$$




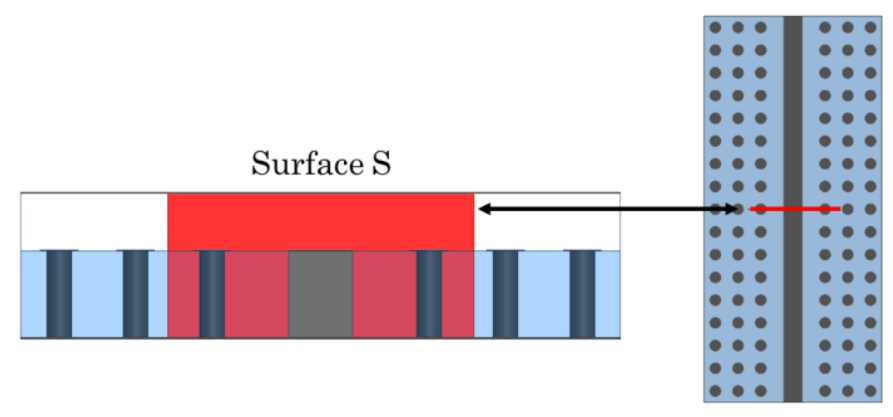

Figure 2.29. Surface used in simulations to obtain the Poynting vector.

\section{4) Fourth approach}

This method is based on the relationship between absolute value of the voltage and two times the power obtained as it is shown in (7).

$$
\mathrm{Z}_{\mathrm{PV}}=\frac{|\mathrm{V}|^{2}}{2 \mathrm{P}}
$$

Now, we compute the values of the power flow in a surface $\mathrm{P}$, the current voltage $\mathrm{V}$ and the longitudinal current $\mathrm{I}$ in the middle of the structure. In Table 2.3 , the results of the characteristic impedance are illustrated using the four methods described previously. It is well known that for a TEM or quasi-TEM mode, the three methods must give closely-related results. In our case, gap waveguide is designed with dielectric, the medium is inhomogeneous, so it is not recommended the use of the approximation 1 (comparison with a half-stripline).

Table 2.3. Values of the characteristic impedance calculated by means of the four methods at the center of the structure.

\begin{tabular}{|cc|}
\hline Approach & Characteristic impedance \\
\hline 2 & 123.7 \\
3 & 121.4 \\
4 & 126.03 \\
\hline
\end{tabular}

The impedance $\mathrm{Z}$ observed in the wave port of the HFSS simulation is 94.5 $\mathrm{ohm}$. As it is expected, the three approaches offer a similar value of the characteristic impedance. Differences of the data can be explained as the mode propagated is an approximation of a quasi-TEM mode, therefore it is normal to have little variations of the characteristic value. Now, since the media exhibits a longitudinal periodicity and impedance may vary along the line, we consider the analysis of the characteristic impedance for five possible cross-sections along the 
line spaced $\lambda_{0} / 4$ to calculate the $\mathrm{Z}_{0}$ value of the microstrip gap waveguide as it is seen in Figure 2.30. We compute the impedance value for each cross-section using the three methods. Results are depicted in Table 2.4 and they are represented in Figure 2.31.
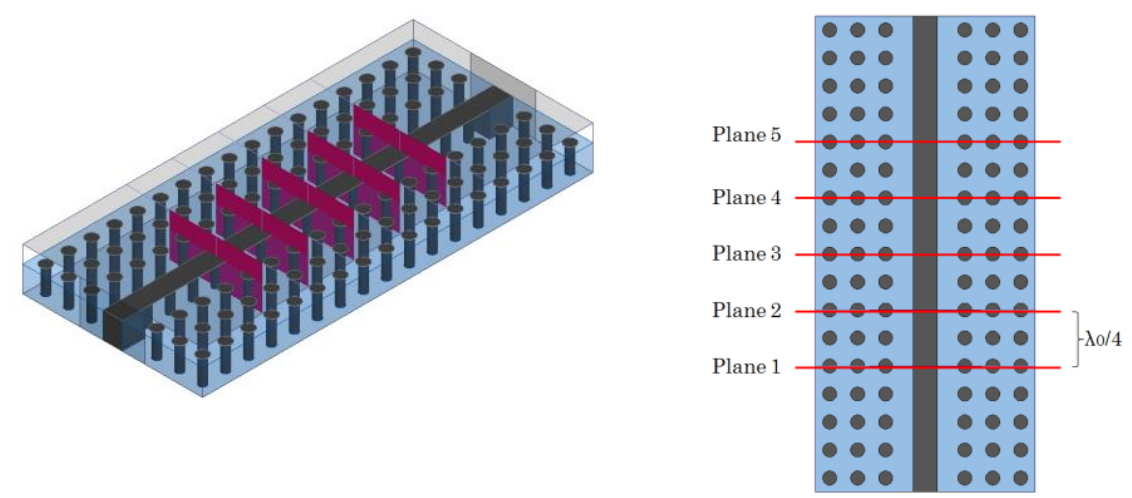

Figure 2.30. Five cross-sections to evaluate the characteristic impedance value.

Table 2.4. Result of the three methods in 3 cross-section positions.

\begin{tabular}{|cccc|}
\hline Plane & Approach 2: ZvI & Approach 3: ZPI & Approach 4: ZPV \\
\hline 1 & 127.99 & 125.48 & 130.56 \\
2 & 140.69 & 138.45 & 142.95 \\
3 & 123.7 & 121.4 & 126.03 \\
4 & 142.99 & 140.48 & 145.55 \\
5 & 123.57 & 120.89 & 126.3 \\
\hline
\end{tabular}

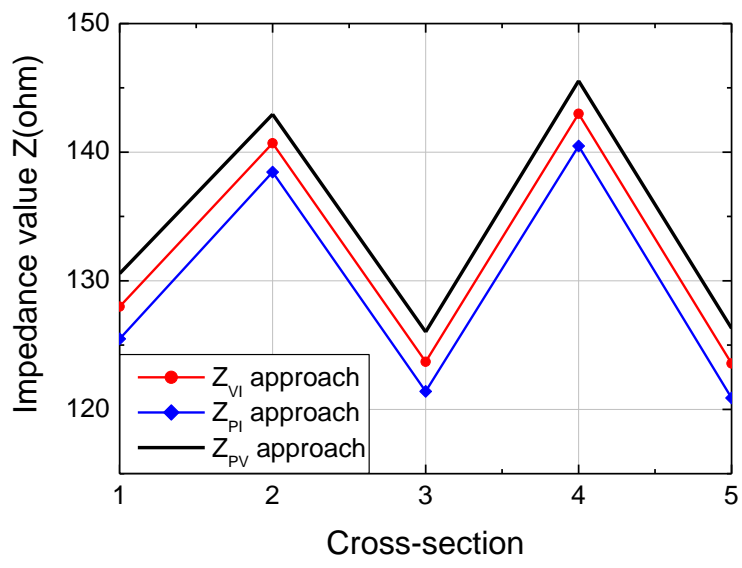

Figure 2.31. Computed impedance value with the 3 methods for each cross-section. 
In this section, several approaches to obtain the characteristic impedance of the gap waveguide have been presented for the case of the gap waveguide with dielectric. We can estimate the characteristic impedance by the average of the three approaches.

\subsection{Study of losses of the microstrip gap waveguide}

One of the benefits of the gap waveguide is its low loss. In [53] a numerical study of losses in ridge gap waveguide in terms of quality factor was presented. Results show that in comparison with ideal rectangular waveguide and microstrip line, gap waveguide presents lower loss since the quasi-TEM mode is allowed to propagate along the ridge without any need for dielectric.

A preliminary study of loss of the microstrip gap waveguide (gap waveguide with dielectric) was done in [54], with the Q-factor for this configuration evaluated at $10 \mathrm{GHz}$. In our work, the operating frequency is $60 \mathrm{GHz}$. We propose some design considerations for this topology at this frequency range.

\subsubsection{Dimensioning and theoretical study}

Rectangular waveguides have high Q-factors, but fabrication may become complex and expensive for millimetre-wave designs. In the other hand, planar transmission lines have losses due to the use of dielectric materials and the unwanted leakage loss or associated problems related to packaging and shielding. Finally, SIW structures have been presented as an attractive technology for applications in the millimetre-wave band. Furthermore SIW can be made thinner than gap waveguide. However, the conductive loss becomes significant especially in the millimetre-wave band. Also, in SIW the wave propagation takes place within the dielectric, hence increasing propagation loss.

Gap waveguide originally does not require the use of any dielectrics, it is naturally shielded and has no radiation loss. The bed of nails forming the HIS can easily stop the leakage loss; it has a high Q-factor in comparison with the reduced $\mathrm{Q}$-factor of standard waveguides at high frequency because it has not any metallic joints.

The study of the gap waveguide losses will be done by the computed Q-factor. $\mathrm{Q}$-factor indicates the rate of energy loss relative to the stored energy of the resonator. As it is known, higher Q-factor implies low loss. 
One method to compute the $\mathrm{Q}$-factor of the resonator consists on the use of the HFSS Ansoft Design Eigenmode solver, which directly calculates the unloaded Q from the eigenmodes of the structure. In this work, a short circuit resonator is considered. Figure 2.32 illustrates the design of the short-circuit gap waveguide resonator considered for simulations. The structure is composed by the metal ridge surrounded by a pattern of periodic metal pins surface. The top and bottom are metallic plates and the air gap always fulfils the condition hgap $<\lambda_{0} / 4$ for any frequency. Results using two LTCC tapes, Ferro A6-M and ESL41110 have been generated for comparison purposes. All the materials are modelled including their loss specifications (both conductor and dielectric loss).

Usually, the unloaded Q-factor of a waveguide resonator can be expressed as:

$$
\frac{1}{Q_{\text {unloaded }}}=\frac{1}{\mathrm{Q}_{\text {metal }}}+\frac{1}{\mathrm{Q}_{\text {dielectric }}}+\frac{1}{\mathrm{Q}_{\text {leakage }}}
$$

The dielectric loss is related to the volume of the dielectric. Metal loss is caused by the volume of the resonator and the conductivity of the metallic parts. The leakage loss is related to leaking of energy through the tiny gaps formed due to mechanical imperfections of the metal split block sections. The lowest $\mathrm{Q}$ value dominates in determining the unloaded $\mathrm{Q}$ of the resonator.

If we consider low loss transmission line resonators with propagation constant $B$ and attenuation constant $\alpha$, the unloaded $Q$-factor can be defined as:

$$
Q=\frac{\beta}{2 \alpha}
$$

From the knowledge of the $\mathrm{Q}$-factor it is possible to extract the attenuation constant of the transmission line of the resonator. The boundary conditions and material properties used in the simulations are defined in Figure 2.32.

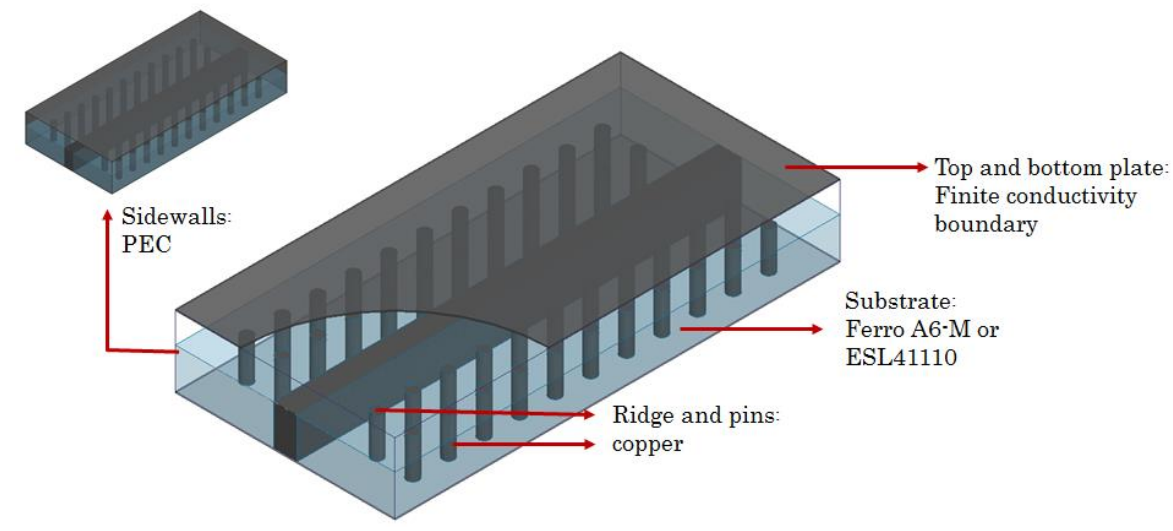

Figure 2.32. Gap waveguide short-circuit resonator using Eigenmode solver of HFSS. 
In [55] a study of loss of the ridge and groove gap waveguide designed with metal pins was shown. That analysis did not include loss in the dielectric and it was carried out at $13.3 \mathrm{GHz}$. In this study, we propose a characterization of the line around an operating frequency of $60 \mathrm{GHz}$. The resonant length has been chosen in order to obtain a third-order resonance at around $60 \mathrm{GHz}$ with the aim to study loss of gap waveguide in the millimeter-wave range. For this reason, the resonant length of the ridge should be 1.5 wavelengths that correspond to $7.5 \mathrm{~mm}$. The wavelength is $\lambda_{0}$ because in the linear part of the quasi-TEM mode propagation occurs through the air gap. As it is explained above, in this study, two LTCC substrates are considered. Their electromagnetic properties are shown in Table 2.5. These values are defined by the manufacturer. The choice of these specific substrates is based on the fact that Ferro A6-M is a material available in the LTCC laboratory of the Universitat Politècnica de València and ESL41110 is the material employed in the LTCC laboratory of Télécom Bretagne. As we can see, ESL41110 tape has more loss than Ferro A6-M. In a recent work done in Télécom Bretagne [56], the choice of ESL41110 tape from the American manufacturer ESL is explained and justified. In this work, four classical tapes are compared: ESL41110, Ferro A6-M, Dupont 951 and Heraeus CT2000. This study is based on the operating frequency range and the interest for the use of low relative permittivity for the design of printed lines. Conclusions of this study show that ESL41110 exhibits the lower permittivity and it allows operating at a higher frequency.

Table 2.5. Characteristics of the two LTCC tapes used in this study.

\begin{tabular}{|ccc|}
\hline LTCC tape & $\boldsymbol{\varepsilon r}$ & $\tan \delta$ \\
\hline Ferro A6-M & 5.9 & 0.002 \\
ESL41110 & 4.2 & 0.004 \\
\hline
\end{tabular}

In Figure 2.33, the top view of the short-circuit gap waveguide resonator is represented. The electromagnetic field for the third-order mode of resonance is depicted in Figure 2.34. A comparison between mushrooms and pins has been performed in order to evaluate the repercussion in the $\mathrm{Q}$-factor value. The pin diameter is $200 \mu \mathrm{m}$, the path diameter is $300 \mu \mathrm{m}$, the width of the ridge is wridge $=0.4 \mathrm{~mm}$, the height of the air gap is $450 \mu \mathrm{m}$, the height of the pins is 675 $\mu \mathrm{m}$ corresponding to nine LTCC layers, the period of the pins $\mathrm{p}=600 \mu \mathrm{m}$ and the resonant length is $7.5 \mathrm{~mm}$. In the simulations lossy materials have been 
considered, being copper the conductor employed. As it was been explained in the previous section, the HIS can be created of different periodic shapes like metallic mushrooms or pins. A comparison of the impact of the choice of the shape in the Q-factor is depicted in Table 2.6.

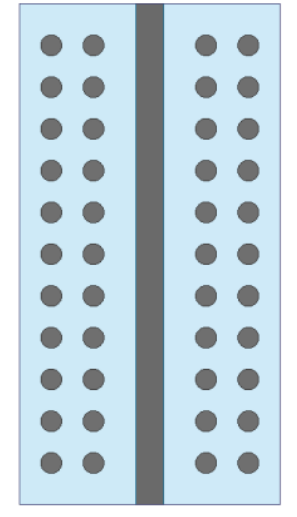

Figure 2.33. Top view of the shortcircuit gap waveguide resonator.

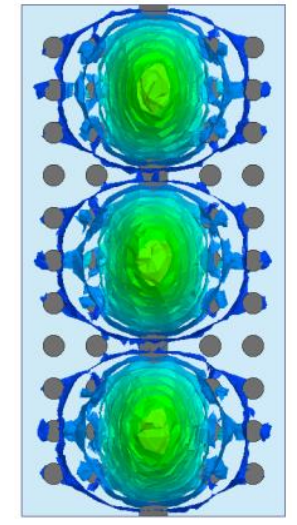

Figure 2.34. Excited third-order resonant mode.

Table 2.6. Comparison of $\mathrm{Q}$-factor as a function of mushroom-shape and pin surface.

\begin{tabular}{|c|ccc|ccc|}
\cline { 2 - 7 } \multicolumn{1}{c|}{} & \multicolumn{3}{c|}{ MUSHROOMS } & \multicolumn{4}{c|}{ PINS } \\
\cline { 2 - 7 } & Q-factor & $\begin{array}{c}\text { Frequency } \\
(\mathrm{GHz})\end{array}$ & $\mathrm{a}(\mathrm{dB} / \mathrm{m})$ & Q-factor & $\begin{array}{c}\text { Frequency } \\
(\mathrm{GHz})\end{array}$ & $\alpha(\mathrm{dB} / \mathrm{m})$ \\
\hline ESL41110 & 318.1 & 60.8 & 1.97 & 322.47 & 61.1 & 1.95 \\
\hline Ferro A6-M & 451.6 & 54.65 & 1.39 & 459.62 & 54.99 & 1.37 \\
\hline
\end{tabular}

The computed results are shown in Table 2.6 and we can observe that with Ferro A6-M, a higher Q-factor value is obtained that can be explained from the lower value of dielectric loss showed. Despite this fact, henceforth, for this study ESL41110 will be considered because its low permittivity and the possibility to create the largest microstrip line relative to the other tapes [56].

From this point, a periodic pattern of mushrooms will be considered to create the HIS surface. It is important also to determinate difference in terms of loss of three configurations: ridge gap waveguide (Figure 2.35-a), microstrip gap waveguide with periodic pins connecting the strip to ground plane (Figure 2.35-b) and without this row of periodic pins shorting the strip (Figure 2.35-c). 


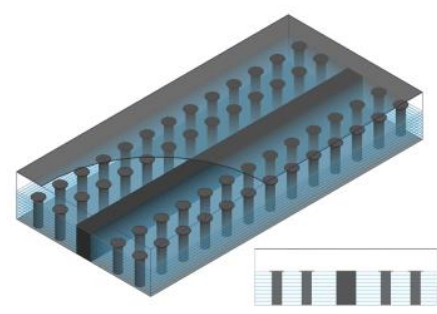

$\mathrm{Q}=317.08$

$(\mathrm{f}=60.8 \mathrm{GHz})$

a) Ridge gap waveguide

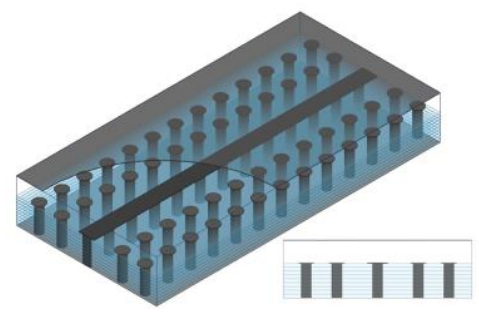

$\mathrm{Q}=253.72$

$(\mathrm{f}=59.02 \mathrm{GHz})$

b) Strip with pins below

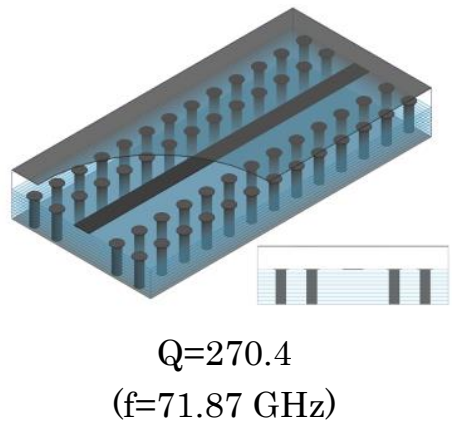

c) Strip without pins below

Figure 2.35. Three configurations based on gap waveguide concept.

The three configurations are illustrated in Figure 2.36. Computed results are obtained by means of the eigenmode HFSS solver. In all cases, lossy materials were considered. In the configuration without pins below the strip (Figure 2.35-c), the $\mathrm{Q}$-factor exhibits a higher value than in the case with pins below (Figure $2.35-b)$. This is because in fact two modes are propagating at this frequency, one between the strip and the upper metal plate, and another one between the strip and the lower metal plate as it is depicted in Figure 2.36-b. Therefore, we cannot consider this value for the comparison because it is not under the same propagation conditions.

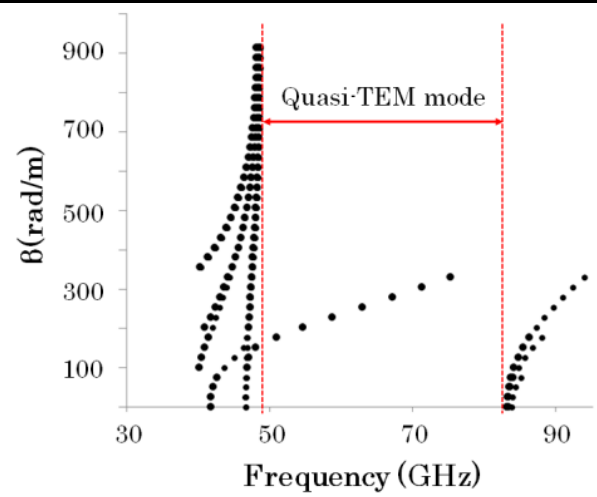

Configuration $2:$ transmission line with pins below

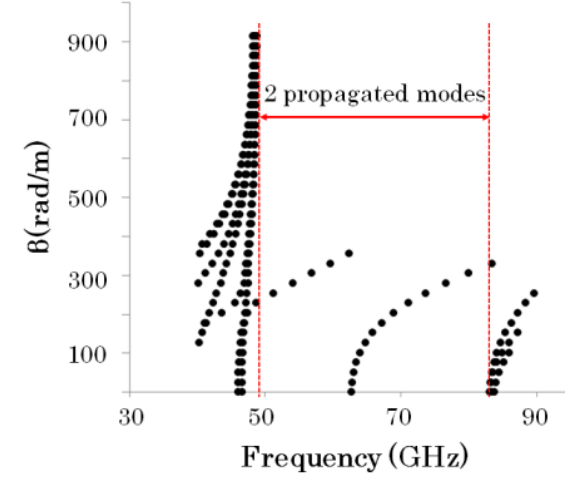

Configuration $3:$ transmission line without pins below

Figure 2.36. Dispersion diagram of the configuration with strip and pins below and without pins, respectively.

Ridge gap waveguide configuration offers a higher $\mathrm{Q}$-factor value. The configuration transmission line without pins below offers a high $\mathrm{Q}$-factor in comparison with the transmission line with pins. 


\subsubsection{Influence of the gap height in the $\mathrm{Q}$-factor value:}

The quasi-TEM mode as it has been described in the introduction of this chapter is excited in the air gap. Thus, the gap height plays an important role in the $\mathrm{Q}$-factor value. In [53], an analysis of the $\mathrm{Q}$-factor is presented varying the gap height in the classical ridge gap waveguide without dielectric. Typically, the introduction of a dielectric in the pin surface should not affect the Q-factor significantly because the role of this surface is to create the HIS condition. For our study, we consider the microstrip gap waveguide with pins below and the ESL41110 substrate.

Results are shown in Table 2.7 and Figure 2.37. As it was expected, the Qfactor increases as gap height increases. The current density being less concentrated when the gap between the ridge and the upper lid is larger. Nevertheless, at approximately $\lambda_{0} / 8$ at $60 \mathrm{GHz}$, it is observed that this growth is less significant. This can be due to the fact that as the gap height increases, the maximum frequency of the stop-band decreases, as shown in Figure 2.38. From $\lambda$ o/8, the resonant frequency $60 \mathrm{GHz}$ is out of the stop-band region (because the maximum frequency has decreased more than $60 \mathrm{GHz}$ ). Therefore, in this area there is a multimode behavior and losses increases due to the propagation of other modes.

Table 2.7. Q-factor and attenuation constant for each air gap height

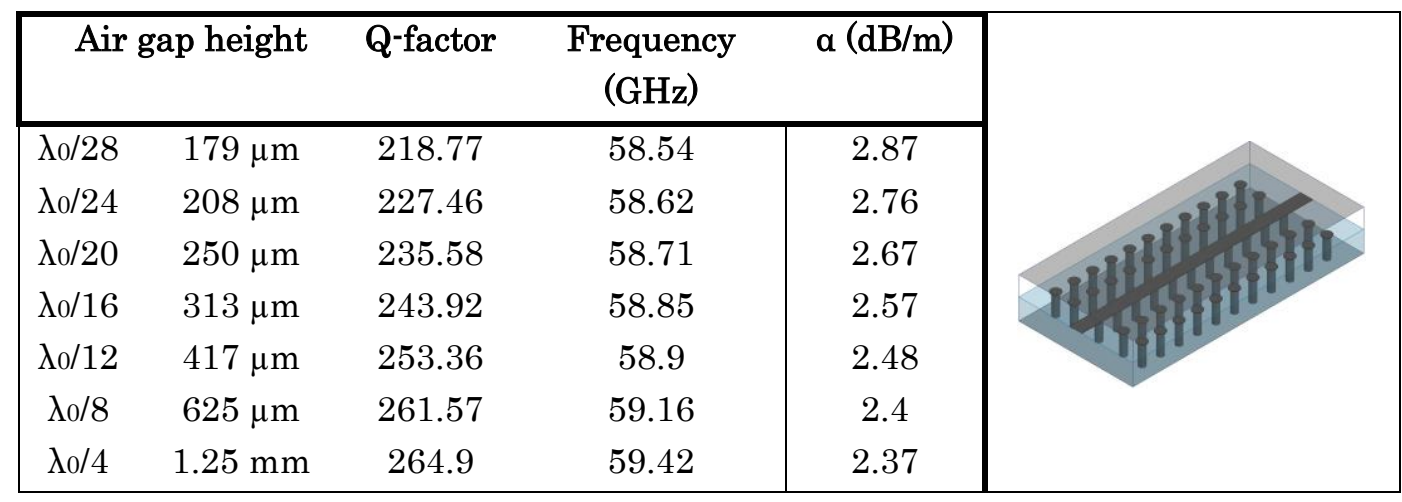




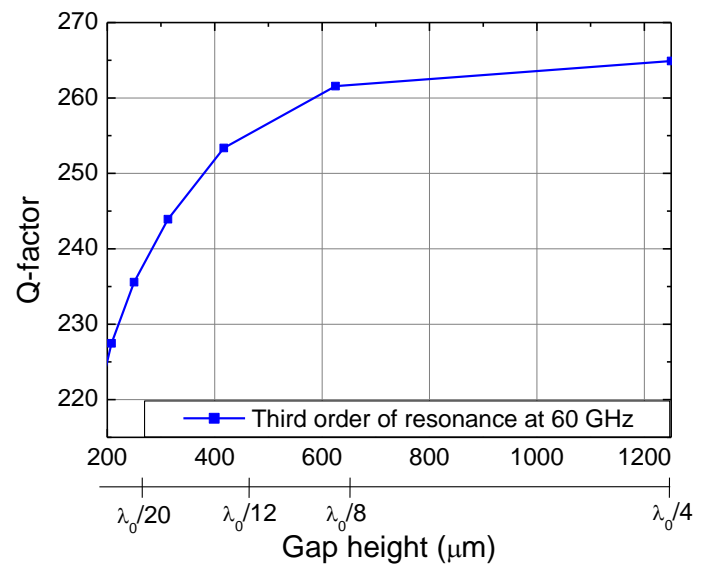

Figure 2.37. Computed $\mathrm{Q}$-factor versus gap height at $\mathrm{f}=60 \mathrm{GHz}$.

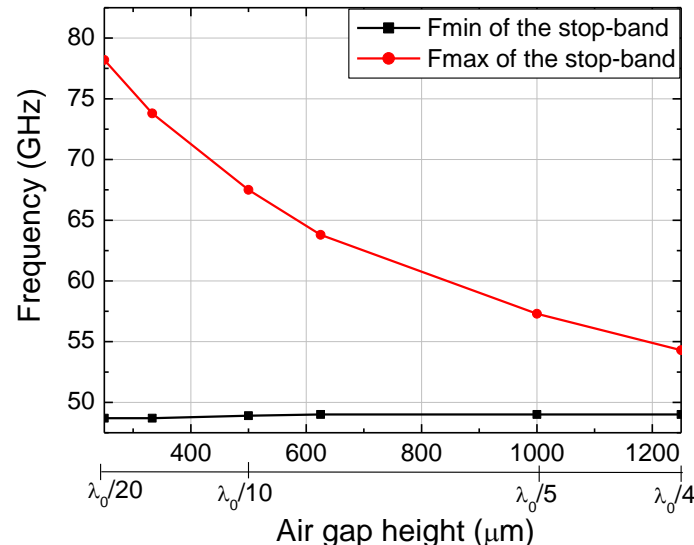

Figure 2.38. Computed minimum and maximum frequency of the stop-band.

For a further study, we consider the three topologies presented in Figure 2.35. We compare the $\mathrm{Q}$-factor value as a function of the gap height in Figure 2.39, considering all loss in the materials. 


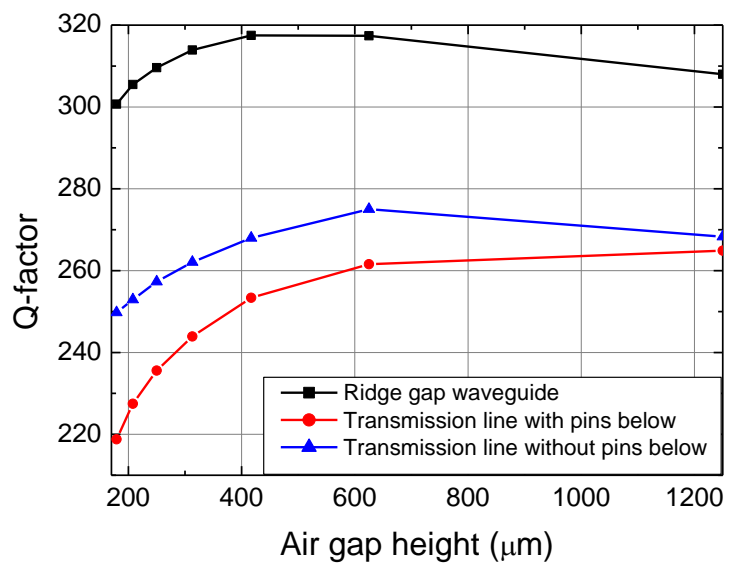

Figure 2.39. Computed Q-factor versus gap height for the three configurations considering dielectric and conductor losses.

The difference between the $\mathrm{Q}$-factor obtained with the transmission line with and without pins below is mainly due to the impact of the conductor loss as it is depicted in Figure 2.40. In this figure, no loss in the dielectric is considered, only loss in the metallic pins and in the upper and lower metal plate is taken into account.

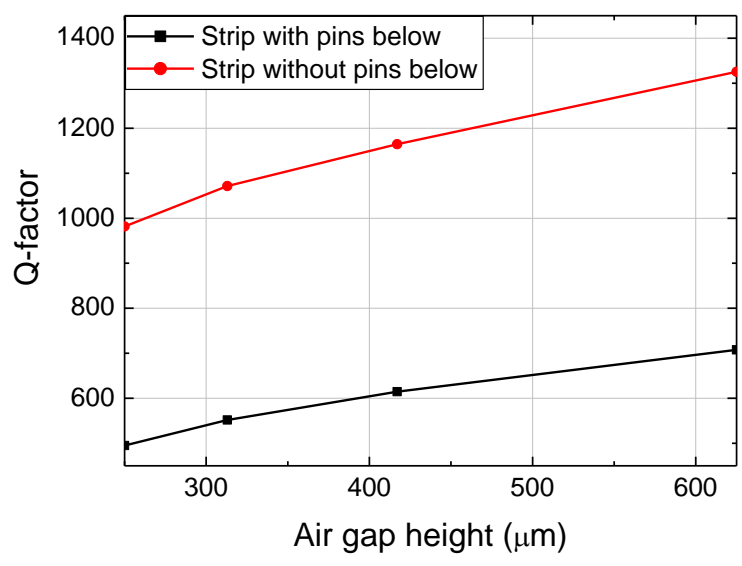

Figure 2.40. Computed Q-factor due to the conductor loss in function of the gap height.

The E-field distribution for the three configurations is represented in Figure 2.41. In all cases, the larger intensity of the E-field is between the ridge or strip and the upper metal plate (propagation takes place in the air gap). It is important 
to mention, that the fact to introduce pins below the strip of the gap waveguide stops the mode propagating between the ground plane and the strip.

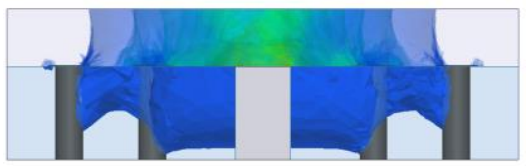

Ridge gap waveguide

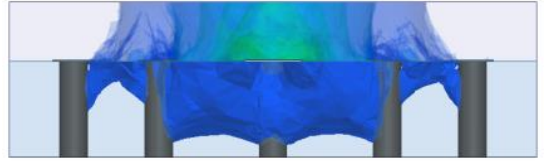

Transmission line with pins below

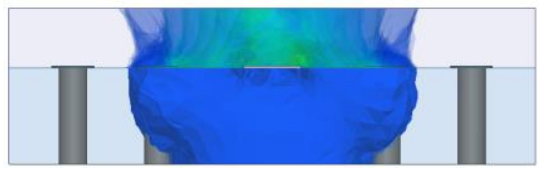

Tx line without pins below

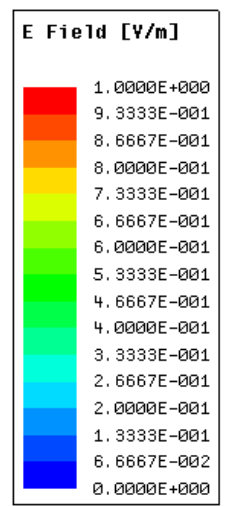

Figure 2.41. Front view of the E-field propagated in the three configurations for the third order mode of resonance for hgap $=417 \mu \mathrm{m}$ (considering no losses).

In Table 2.8, the results of the computed $Q$-factor and loss a $(\mathrm{dB} / \mathrm{m})$ for the ridge and transmission line with pins below are presented. In the first case, all lossy materials are considered, namely, dielectric with nonzero value for tan $\delta$, and copper as conductor. In the second case, the conductor is an ideal conductor ( $\mathrm{PEC}$ condition) and no loss in the dielectric material $(\tan \delta=0)$. The gap height is fixed at $\lambda_{0} / 12=417 \mu \mathrm{m}$ at $60 \mathrm{GHz}$ and the parameters are the same as those used at the beginning of this section. The attenuation constant a can be extracted using:

$$
\alpha=\frac{\beta}{2 Q_{U}}
$$

where $B$ is the propagation constant $(\beta=2 \pi / \lambda)$.

Table 2.8. Computed results of $\mathrm{Q}$ and a for the three configurations considering: lossy materials, only loss in the dielectric and only loss in the conductor.

\begin{tabular}{|c|cc|cc|}
\hline \multicolumn{1}{|c|}{} & \multicolumn{2}{c}{ RIDGE } & \multicolumn{2}{c|}{ STRIP WITH PINS } \\
\hline & $\mathrm{QU}$ & $\mathrm{a}(\mathrm{dB} / \mathrm{m})$ & $\mathrm{QU}$ & $\mathrm{a}(\mathrm{dB} / \mathrm{m})$ \\
\cline { 2 - 5 } 1. All lossy & 317.48 & 1.98 & 253.36 & 2.48 \\
materials & & & & \\
$\begin{array}{c}\text { 2. Without } \\
\text { conductor loss } \\
\text { 3. Without } \\
\text { dielectric loss }\end{array}$ & 432.83 & 1.45 & 442.19 & 1.42 \\
& & & & \\
\hline
\end{tabular}


The results of the $\mathrm{Q}$-factor of the comparison between ridge gap waveguide and strip with pins below configuration are depicted in Figure 2.42 and in function of the attenuation constant in Figure 2.43.

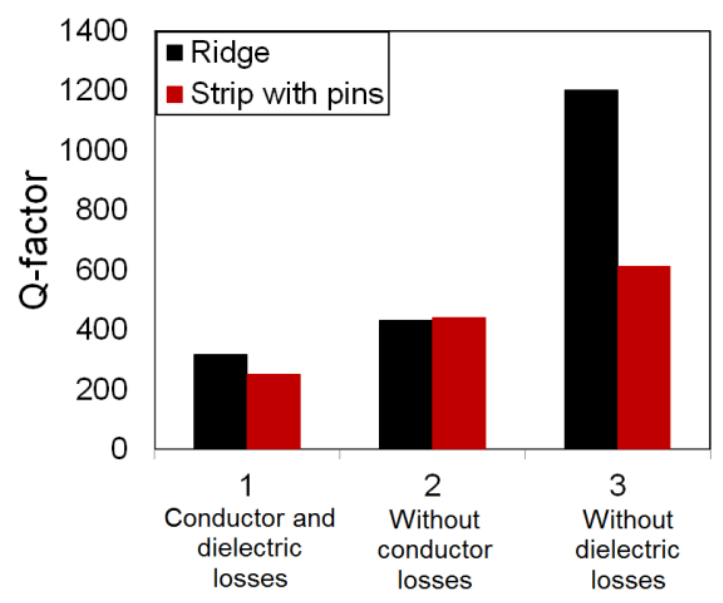

Figure 2.42. Q-factor due to conductor, dielectric or both losses for the ridge and transmission line configurations.

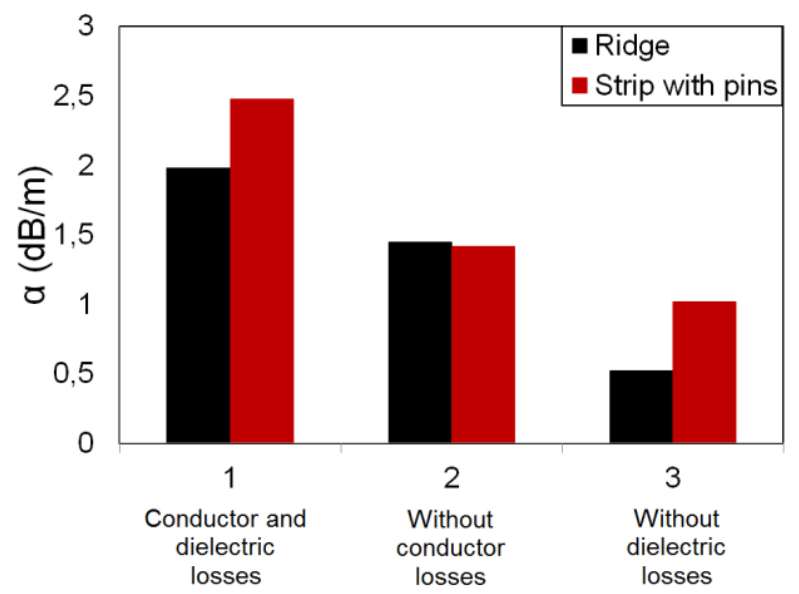

Figure 2.43. Attenuation constant due to conductor, dielectric or both losses for the ridge and transmission line configurations.

As can be observed, the most important contribution to loss is related to the dielectric loss as it is usual at this operating frequency. From these figures, we can extract also that in the configuration where the transmission line had no pins below, the conductor loss plays an important role in the total Q-factor, being this percentage more significant than in the other two configurations. 
Finally, another analysis that is worth performing is the influence of the width of the ridge or strip (wridge) in the Qu-factor. Considering the configuration based on a transmission line with pins below and the HIS created by a periodic surface of mushrooms, the $\mathrm{Qu}$-factor as a function of the wridge is computed. Results are illustrated in Table 2.9 and Figure 2.44. All loss of the materials is included and the gap height is fixed at $417 \mu \mathrm{m}\left(\lambda_{0} / 12\right)$. Results show that as the width of the strip increases, Q-factor increases, a conclusion which is in line, with other classical transmission lines, such as microstrip or stripline where narrow width leads to higher conductor loss.

Table 2.9. Q-factor versus the strip width.

\begin{tabular}{|cccc|}
\hline \multicolumn{4}{|c|}{ TX LINE WITH PINS BELOW } \\
\hline wridge & $\mathbf{Q u}(60 \mathrm{GHz})$ & Freq (GHz) & $\mathbf{a}(\mathrm{dB} / \mathrm{m})$ \\
\hline 0.3 & 248.23 & 59.3 & 2.53 \\
0.4 & 253.36 & 58.9 & 2.48 \\
0.5 & 273.35 & 58.89 & 2.29 \\
0.6 & 286.57 & 58.6 & 2.19 \\
0.7 & 288.77 & 58.12 & 2.17 \\
0.8 & 295.06 & 57.8 & 2.13 \\
\hline
\end{tabular}
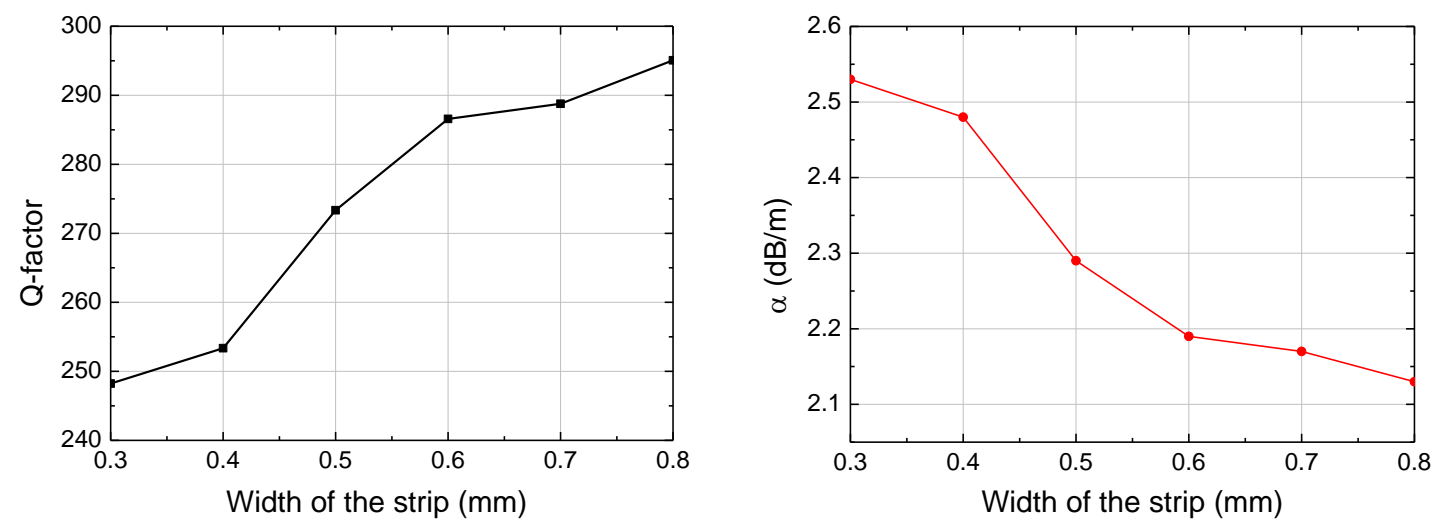

Figure 2.44. $\mathrm{Q}$-factor as a function of the width of the strip. 


\subsection{Conclusion}

In this chapter, principle of operation of the gap waveguide has been presented. At the millimetre-wave band, the technical constraints in terms of dimensions and packaging of the classical guiding structures become more significant. In this context, in recent years the gap waveguide technology has emerged as a new guiding technology that can overcome the disadvantages of this technology.

Firstly, an overview of the gap waveguide concept was presented. The operating principle based on the stop-band region created by a HIS was emphasized. The basic idea of this technology is that the propagation takes place between a metal ridge or strip and an upper metal plate. The HIS stops the propagation, confining in this way the maximum of the E-field in the desired zone.

Secondly, a design of the gap waveguide compatible with PCB or LTCC manufacturing techniques was shown. Numerical studies concerning design and computation of characteristic impedance of these structures were presented. Results allow characterizing this kind of structures and to provide reference values for design.

Finally, an assessment of the losses through the $\mathrm{Q}$-factor of the gap waveguide incorporating dielectric and compatible with PCB or LTCC technology has been introduced. As a result of such characterization it can be concluded that in gap waveguide the influence of many factors is important in the analysis of the $\mathrm{Q}^{-}$ factor, as the distance between ridge and the adjacent row of pins, shape of the elements creating the HIS surface, type of configuration (ridge, strip with or without pins below). An important analysis of the $\mathrm{Q}$-factor as a function of the $\mathrm{p} / \mathrm{d}$ ratio (periodicity of the pins over pin diameter) has been presented. This characterization has been performed to be able to make a posterior comparison with other technologies that are subject to more strict design standards in terms of this p/d ratio, such as SIW technology, as it will be seen in Chapter 3 . 


\section{Chapter 3}

\section{Comparison between Gap Waveguide and SIW technology}

\subsection{Introduction}

In the previous chapter, we have demonstrated the advantages of the gap waveguide for the design in the $\mathrm{mm}$-Wave band due to its low losses. In this chapter, we present a comparative study between gap waveguide with dielectric and Substrate Integrated Waveguide (SIW) structures.

The implementation of SIW components in the $\mathrm{mm}$-wave frequency range will require the use of novel manufacturing techniques with the aim to reduce the size, improve the bandwidth, and especially minimize the losses. Typically SIW technology has been validated using PCB (Printed Circuit Board) techniques due to its low cost and simplicity. Nevertheless, to provide more design flexibility while maintaining the advantage of planar and low-cost fabrication, these structures can be based on multilayered configurations. In this context, in recent years, new technologies such as LTCC or HTCC (High-Temperature Co-fired Ceramic) have emerged as interesting techniques for the design of components in the $\mathrm{mm}^{-w}$ ave band. They will open new scenarios for the applicability of SIW structures. LTCC is presented as a candidate for the design of SIW at the $\mathrm{mm}^{-}$ 
wave band and as it was shown in Chapter 2, the design of the gap waveguide using LTCC was validated. In this chapter, we propose an exhaustive comparative study of gap waveguide and SIW at $170 \mathrm{GHz}$. We have chosen this operating frequency to define the limits of both structures, especially in terms of losses.

This chapter is organized as follows. Firstly, the methodology for the three principal mechanisms of losses in SIW and gap waveguide is presented in Section 3.1. Then, the modeling and the optimized design for both technologies (SIW and gap waveguide) is discussed in Section 3.2. In Section 3.3, the leakage study in terms of losses is presented. Finally, the study of conductor and dielectric losses is presented in Section 3.4.

\subsection{Study of losses - Methodology}

The extremely-high frequency range, EHF, covers the band between 30 and 300 $\mathrm{GHz}$, and the principal applications are in radio astronomy and remote sensing. Furthermore, as it has been explained in Chapter 1, there is an interest to increase in frequency for microfluidic applications. In this regard, the principal guiding structures at these frequencies are subject to packaging problems and losses [57]. Commonly, SIW structures are being used also in the millimeter-wave range. With this technology, the selection of the via spacing should guarantee the propagation and reflection characteristics of the SIW.

However, the problems concerning the different types of losses that a SIW structure undergoes, can involve limitations in its manufacturing.

With the purpose to find a guiding structure more suitable at this range of frequency, a comparative study between gap waveguide and SIW is suggested in this chapter. Both structures will be designed with LTCC technology as it allows working in this band due to its advantages. These merits are: compactness, higher thermal conductivity than those of dielectric substrate, and the similar thermal coefficient of expansion (TCE) to that of silicon and GaAs, that implies good reliability.

The use of LTCC technology for the Gap Waveguide manufacturing may cause restrictions in the design such as pin diameter, substrate layer thickness, permittivity, etc. In our design, the LTCC substrate used for the design will be Ferro A6-M, in order to subsequently perform a comparison between an example 
of SIW published in [58] and gap waveguide structures. This substrate is characterized by a permittivity $\varepsilon r=5.9, \tan \delta=0.002$ (at $100 \mathrm{GHz}$ ), and a layer thickness $0.095 \mathrm{~mm}$ after the co-fired process.

The principal mechanisms of losses in guiding structures are: conductor, dielectric and leakage loss. A study based on the $\mathrm{Q}$-factor value of two resonators based on SIW and gap waveguide technology is proposed in this section. In the publication [59], computed losses and Q-factor of a microstrip line and gap waveguide (without dielectric) have been already compared. These results show that ridge gap waveguide exhibits less losses than microstrip lines due to the propagation medium, in microstrip line waves are propagated in the dielectric, while in gap waveguide in the air.

As it has been explained in Chapter 2, one of the best approaches to characterize losses of a guiding structure is the $\mathrm{Q}$-factor value that is directly related to the losses value. Losses for the microstrip gap waveguide (with dielectric) has been reported in [60]. This study is very interesting, hence it is demonstrated that microstrip gap waveguide has less loss in comparison with the conventional microstrip line. The reasons are that there is no substrate in the air gap region where the propagation takes place, and that gap waveguide are more flexible to use wider metal strip without having problems with radiation and surface waves. In our work, we are interested to compare with SIW structures, and a specific study in terms of leakage losses will be presented.

In this case we compare the gap waveguide and SIW structure at $170 \mathrm{GHz}$. First, the propagation in a gap waveguide takes place in air, whereas is in the dielectric in SIW. Hence, a priori, propagation losses in SIW are higher than in the gap waveguide.

The total Q-factor in the resonators based on the SIW and gap waveguide can be calculated as it is shown in (3.1). Its attenuation comes from dielectric loss, conductor loss and electromagnetic wave leak.

$$
\frac{1}{\mathrm{Q}}=\frac{1}{\mathrm{Q}_{\mathrm{c}}}+\frac{1}{\mathrm{Q}_{\mathrm{d}}}+\frac{1}{\mathrm{Q}_{\mathrm{L}}}
$$

Qd: Q-factor due to dielectric loss

Qc: Q-factor due to conductor loss

$\mathrm{Q}_{\mathrm{L}}$ : $\mathrm{Q}$-factor due to electromagnetic wave leak 
In a SIW resonator, the choice of some geometrical parameters, namely the substrate thickness $\mathrm{h}$, the diameter $\mathrm{d}$ of the metal vias, and their longitudinal spacing $p$, can minimize the losses. The metallic pin diameter and separation between vias makes the radiation loss negligible. The diameter and the periodicity of the vias impact directly the leakage losses. Via thickness, h, impacts on the conductor losses (increasing $\mathrm{h}$ will contribute to conductor loss decrease). Its diameter also impacts on the conductor loss (conductor loss slightly decreases when increasing the diameter).

In this chapter, a study of the three mechanisms of losses in gap waveguide and SIW is done. The approach to obtain numerical values of losses is depicted in the flowchart of Figure 3.1. The study of leakage losses will be done by means of the transmission parameters of the structure. Computed results will be obtained considering neither conductor nor dielectric losses, considering PEC conductors and dielectric with $\tan \delta=0$, respectively. The value of the conductor and dielectric losses will be obtained by means of the Q-factor of a RLC resonator model. Leakage loss impacts also in the $\mathrm{Q}$-factor, however in terms of simulation using the Eigenmode of commercial solver and with the design of resonators we can study only the impact of the conductor and dielectric losses.

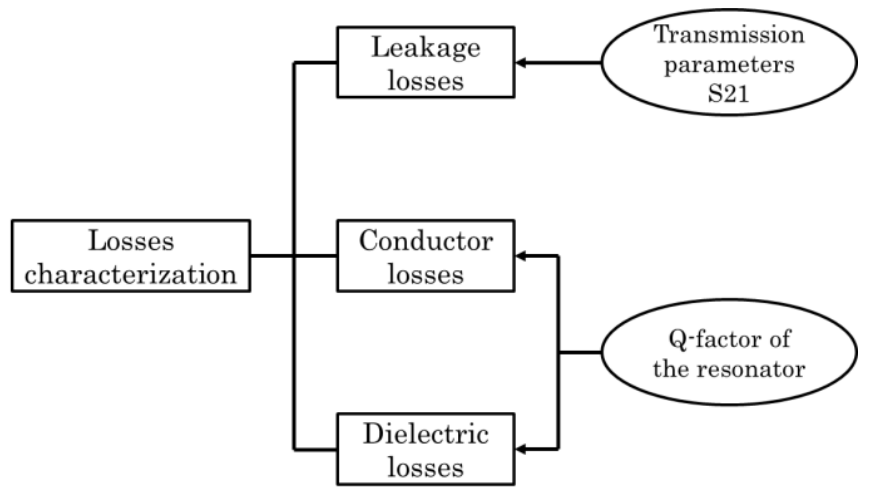

Figure 3.1. Flowchart diagram of the losses study procedure.

\subsection{SIW and gap waveguide design}

\subsubsection{Design of the SIW structure}

Substrate Integrated Waveguide or post-wall waveguide has been presented for the first time in 1994 [61]. Since that time, SIW components have been developed and they are shown as a promising candidate for $\mathrm{mm}^{-}$wave applications. SIW 
technology is fabricated by two rows of conducting cylinders (vias) embedded in a dielectric substrate that electrically connect two metal plates (Figure 3.2).

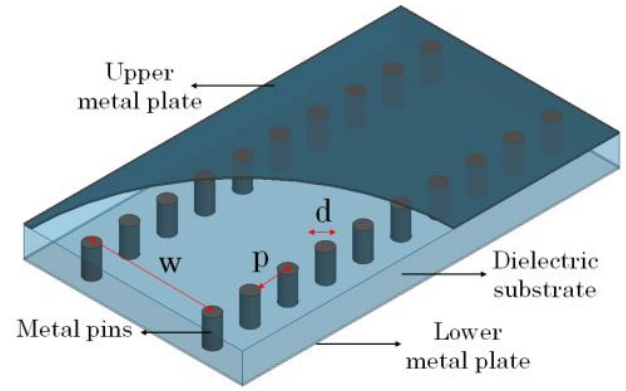

a)

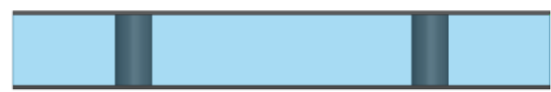

b)

Figure 3.2. SIW technology. a) Three-dimensional view of a SIW structure; b) Front view of SIW.

SIW structures have similar properties to the conventional rectangular waveguides, although, they have the advantages of the traditional waveguide, such as low radiation loss, high $\mathrm{Q}$-factor if lossless dielectric are considered and high power capacity. They are compatible with printed circuit board (PCB) and low temperature co-fired ceramic (LTCC) as manufacturing techniques.

Deslandes and $\mathrm{Wu}$ [62] have developed a method for determining the complex propagation constant of a SIW and design rules for the choice of the via diameter and the via spacing using the concept of surface impedance to model the rows of conducting cylinders which act as sidewalls in a SIW.

SIW only allows the propagation of TEno modes (transverse electric) modes. TM modes are not supported by SIW, due to the gaps between metal vias because the transverse magnetic fields determine longitudinal surface currents, which are subject to strong radiation due to the presence of the gaps [63]. The fundamental mode of SIW is similar to the TE10 mode of a rectangular waveguide.

SIW structures exhibits three mechanisms of loss: conductor losses (due to the finite conductivity of metal walls), dielectric losses (due to the lossy dielectric material) and radiation losses (due to the energy leakage through the gaps). The SIW design procedure is described in [8]. To ensure that the radiation loss and the leakage loss are kept at a very low level, two rules have been explained in [64] and they are shown in (3.2) and (3.3), where $\mathrm{p}$ is the spacing between vias, $\mathrm{d}$ is the via diameter, $\mathrm{c}$ is the speed of light in vacuum. 


$$
\frac{\mathrm{p}}{\mathrm{d}}<2
$$

$$
\mathrm{d}<\frac{\lambda_{\mathrm{g}}}{4}
$$

with $\lambda_{\mathrm{g}}=\frac{2 \pi}{\sqrt{\frac{\varepsilon_{\mathrm{r}(2 \pi \mathrm{f})^{2}}}{\mathrm{c}^{2}}-\left(\frac{\pi}{\mathrm{a}}\right)^{2}}}$.

In SIW technology, the selection of the via spacing should guarantee the propagation and reflection characteristics of the SIW. However, the problems concerning the different types of losses that a SIW structure undergoes, can involve limitations in its manufacturing.

To show this impact with a real example, the frequency=170 $\mathrm{GHz}$, the dielectric permittivity $\varepsilon r=5.9$ and the guided wavelength $\lambda \mathrm{g}=1 \mathrm{~mm}$ are fixed. The maximum value of the via diameter should be smaller than $\frac{\lambda_{\mathrm{g}}}{5}$ according to [64], in the case of operating frequency $170 \mathrm{GHz}$, diameter should guarantee $\mathrm{d}<206 \mu \mathrm{m}$. Combining (1) and (2) and fixing $d \min =\lambda g / 5, \varepsilon r=5.9$, width of the SIW equal to $612.5 \mu \mathrm{m}$, we can represent the maximum distance between vias and the minimum pin diameter as a function of the frequency (Figure 3.3).

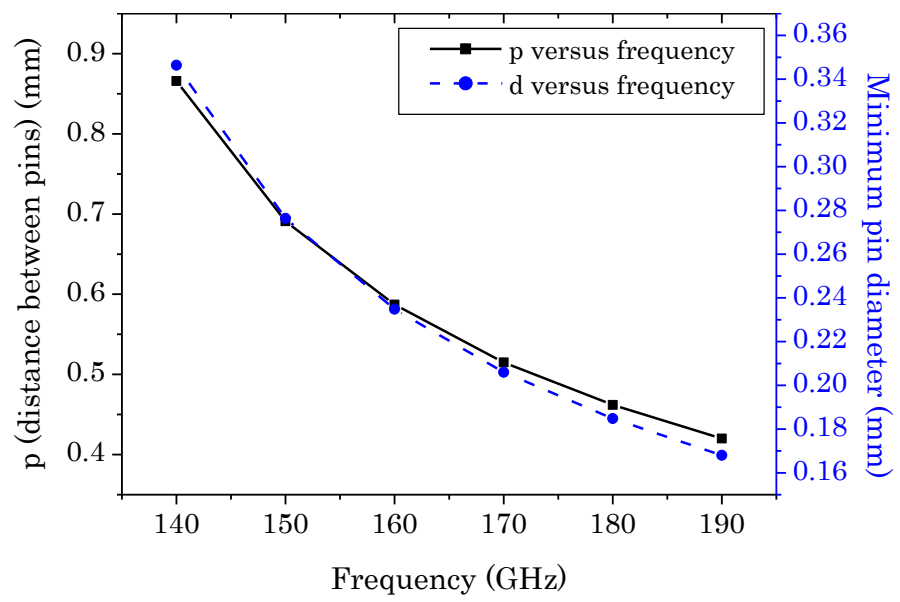

Figure 3.3. Relationship between operating frequency $(\mathrm{GHz})$ and the minimum distance between vias $\mathrm{p}(\mathrm{mm})$ and the minimum via diameter in SIW structures.

This relationship expresses how the distance between vias should be smaller as frequency increases. Furthermore, the minimum value of the via diameter presents the same evolution. At high frequency and depending on the manufacturing technique, an important drawback of the manufacturing techniques consists of making thinner diameters for thinner spacing between them. 


\subsubsection{Design of the gap waveguide structure}

A promising candidate to overcome mechanical limitations of SIW technology is the previously presented gap waveguide technology. The principle of operation of the gap waveguide has been demonstrated already in the Chapter 2 where a design has been performed at $60 \mathrm{GHz}$. In this chapter, following the same development, the design of the gap waveguide has been done at $170 \mathrm{GHz}$.

The front view of the Gap Waveguide in LTCC is shown in Figure 3.4. The condition imposed by the design of the Gap Waveguide is that vias height should be $\lambda \mathrm{g} / 4$ to achieve a HIS behavior. The Ferro A6-M layer thickness is $95 \mu \mathrm{m}$ after the co-firing process. So the LTCC substrate must be formed by two layers, including buried vias and a printed strip on the upper substrate layer.

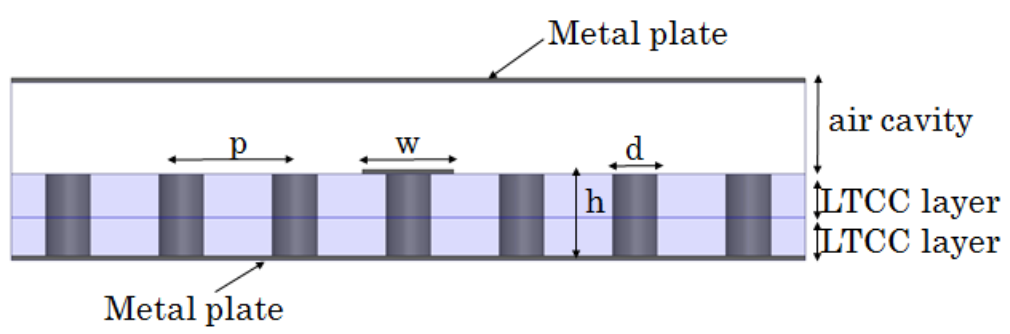

Figure 3.4. Front view of Gap Waveguide with LTCC.

As it has been explained in the introduction, a comparison between SIW and gap waveguide will be done. For this purpose, common dimensions for both technologies have been selected and they are shown in Table 3.1. Another specific dimension for SIW, such as the SIW width, w, is $0.6125 \mathrm{~mm}$ to respect design considerations of SIW. In the gap waveguide structure, the width of the strip is wridge $=0.25 \mathrm{~mm}$ for a characteristic impedance equal to $116 \Omega$. The height of the air gap corresponds to hgap $=0.2 \mathrm{~mm}$.

Table 3.1. Common dimensions of the SIW and gap waveguide design.

\begin{tabular}{|ccc|}
\hline \multicolumn{3}{|c|}{ Gap waveguide and SIW parameters } \\
\hline Via diameter & $\mathrm{d}$ & $100 \mu \mathrm{m}$ \\
\hline Periodicity of the vias & $\mathrm{p}$ & $250 \mu \mathrm{m}$ \\
\hline Via height & $\mathrm{hp}$ & $190 \mu \mathrm{m}(2 \mathrm{LTCC}$ layers $)$ \\
\hline Substrate & LTCC tape: & $\varepsilon \mathrm{r}=5.9$, tan $\delta=0.002$ \\
& Ferro A6-M & layer thickness $=95 \mu \mathrm{m}$ \\
\hline Operating frequency & $\mathrm{f}$ & $170 \mathrm{GHz}$ \\
\hline
\end{tabular}




\subsubsection{Computed results for both technologies}

First of all, we characterize the SIW and gap waveguide described in 3.2.1 in terms of operating frequency range. With this purpose, we compute the dispersion diagram and the propagation characteristics of both structures.

In Figure 3.5, the dispersion diagram of SIW and gap waveguide computed with the Eigenmode solver of HFSS is depicted. SIW structure exhibits a behavior similar to classical rectangular waveguides, where the cut-off frequency of the fundamental mode $\mathrm{TE}_{10}$ is at $120 \mathrm{GHz}$ and the next propagated mode, $\mathrm{TE}_{20}$, is at $240 \mathrm{GHz}$, approximately. The dispersion characteristics and analytical formulas for the TE10 and TE20 modes of SIW are defined in [65].

In the case of the gap waveguide, we can observe in this figure the principle of operation of the gap waveguide. This structure shows a stop-band in the range [151-190] GHz, where a quasi-TEM mode is propagated.

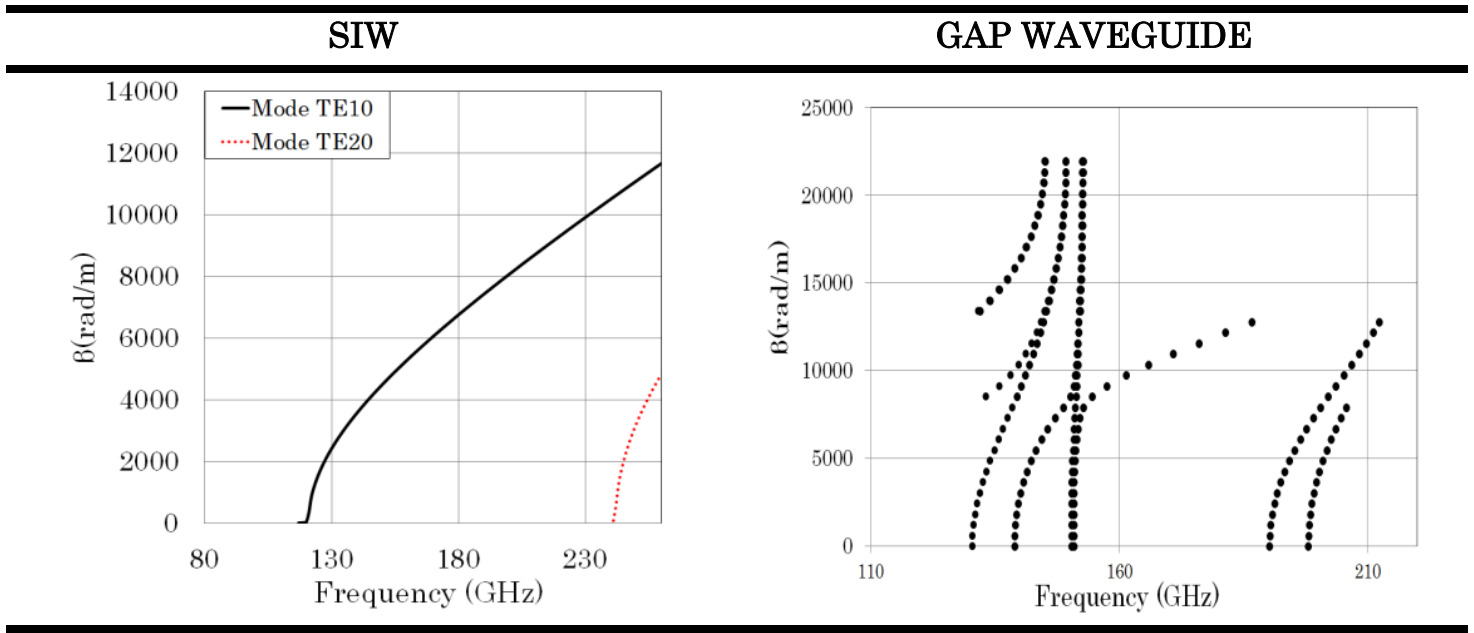

Figure 3.5. Computed dispersion diagram for SIW and gap waveguide, respectively.

In SIW, the mode propagated is TE10, with cut-off frequency fстЕ10 $=120.5 \mathrm{GHz}$. $\mathrm{S}$-parameters are represented in Figure 3.6. The dimensions verify $\mathrm{p} / \mathrm{d}=2.5$. The cut-off frequency for the TE10 mode of SIW is 120.5 GHZ. Next mode, TE20, is propagated from $235 \mathrm{GHz}$. We want to be careful with the computation of the Sparameters with the driven modal of HFSS, because from $230 \mathrm{GHz}$ (Figure 3.6), we can see the contribution of several modes between the two waveport defined for simulations. It does not mean that there is no insertion losses, but the computed parameters from this frequency is the sum of the contributions of some modes. 


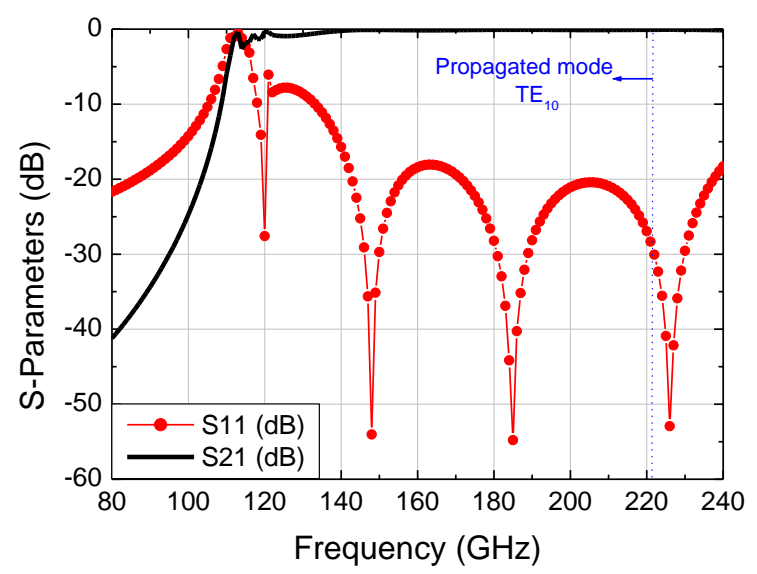

Figure 3.6. Computed S11 and S21 of the SIW with LTCC.

In the case of the gap waveguide, the simulated S-parameters are shown in Figure 3.6. It can be seen that the reflection coefficient is below $-20 \mathrm{~dB}$ over the stop-band range, and the transmission coefficient in this band is about $-0.1 \mathrm{~dB}$. From $190 \mathrm{GHz}$, the frequency stop-band is finished; hence, other modes become propagated. This explains the decrease of the transmission parameter from 190 GHz..

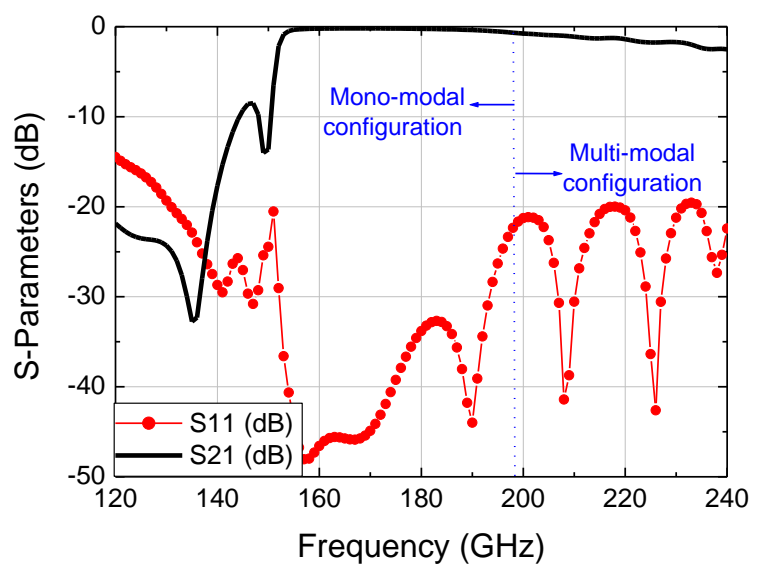

Figure 3.7. Computed S11 and S21 of the LTCC gap waveguide.

\subsection{Leakage losses study}

\subsubsection{Design}

The design of both resonators based on the two technologies has been performed to operate at $170 \mathrm{GHz}$. As it is explained in [66], for SIW, the condition p/d does not guarantee the absence of radiation leakage. It is need to introduce another 
condition $w / d>8$. An analytical formula to calculate the attenuation constant due to radiation leakage has been presented in [67]. For our calculus, some parameters common for the two technologies are fixed and they are listed in Table 3.2.

Table 3.2 Common parameters for both resonators (SIW and gap waveguide).

\begin{tabular}{|ccc|}
\hline \multicolumn{3}{|c|}{ Common parameters for both structures } \\
\hline Via diameter & $\mathrm{d}$ & $100 \mu \mathrm{m}$ \\
\hline Spacing of the vias & $\mathrm{p}$ & $250 \mu \mathrm{m}$ \\
\hline Dielectric & LTCC tape: & $\varepsilon \mathrm{r}=5.9$ \\
& Ferro A6-M & $\begin{array}{c}\text { Tan } \delta=0.002 \\
\text { Layer thickness }=95 \mu \mathrm{m}\end{array}$ \\
\hline $\begin{array}{c}\text { Via height (or } \\
\text { substrate thickness) }\end{array}$ & $\mathrm{hp}$ & $190 \mu \mathrm{m}$ (2 LTCC layers) \\
\hline
\end{tabular}

In addition to these parameters, for each structure, other specific parameters must be defined and are shown in Table 3.3.

Table 3.3. Specific design parameters for SIW and gap waveguide.

\begin{tabular}{|l|ccc|}
\hline \multicolumn{2}{|c|}{ Parameters for SIW } & \multicolumn{3}{|c|}{ Parameters for the gap waveguide } \\
\hline $\begin{array}{c}\text { Distance between } \mathrm{w} 0.6 \mathrm{~mm} \\
\text { the two row of } \\
\text { vias }\end{array}$ & Air gap height & hgap & $200 \mu \mathrm{m}$ \\
\cline { 3 - 4 } & Width of the strip & wridge & $200 \mu \mathrm{m}$ \\
\cline { 3 - 4 } & $\begin{array}{c}\text { Diameter of the } \\
\text { patch }\end{array}$ & $\mathrm{d} 2$ & $150 \mu \mathrm{m}$ \\
\hline
\end{tabular}

\subsubsection{Leakage losses}

Design considerations to reduce leakage loss are one of the most critical points for the design of SIW structures. As it has been explained above, to reduce this losses in SIW, to fulfill the condition $\mathrm{p} / \mathrm{d}<2.5$ is necessary. For this reason, in this study, we are interested in the impact of the ratio distance between vias over via diameter, i.e. the importance of the leakage losses in the Q-factor. Radiation loss is not strongly affected by the substrate thickness (at least, as long as $\mathrm{h}$ is smaller than half wavelength).

To provide numerical value of the leakage losses, the procedure employed is as follows: Considering losses neither in the dielectric nor on the conductor, we compute with HFSS solver S parameters for the dimensions of SIW and gap waveguide described in Table 3.2 and Table 3.3. Once, the S-parameters values 
extracted at $170 \mathrm{GHz}$ and using (4) or (5), we can find the value corresponding to the attenuation constant related to the leakage losses.

For a passive network, and considering no losses in the conductor or in the dielectric, the radiation losses due to the energy leakage through the gaps are the only loss in the structure. We can define the loss value as:

$$
\begin{aligned}
& \alpha_{\text {leakage }}=1-\left|S_{11}\right|^{2}-\left|S_{21}\right|^{2} \\
& \alpha_{\text {leakage }}=10 \log _{10} \frac{\left|S_{21}\right|^{2}}{1-\left|S_{11}\right|^{2}}
\end{aligned}
$$

For a variation in the $\mathrm{p} / \mathrm{d}$ relationship, we can change either $\mathrm{p}$ parameter or $\mathrm{d}$ dimension. The impact of the modification of the diameter is less significant than the spacing between vias variation. If the diameter fulfills the condition $d<\lambda g / 5$ in SIW, the leakage loss will be less significant. However, the reduction of the via diameter is not always possible considering the restrictions of the fabrication process. Moreover, it was verified that the variation of the via diameter has a negligible effect on losses [68]. For this reason, parameter $\mathrm{p}$ is much concerned for leakage loss analysis.

In Table 3.4, the S-parameter value and the attenuation due to the leakage are shown. These values are obtained with the Driven modal of HFSS and considering no dielectric and conductor losses. The leakage attenuation has been calculated with (3.4).

Table 3.4. S-parameters and leakage attenuation computed with the Driven modal at $170 \mathrm{GHz}$ (considering no losses).

\begin{tabular}{|c|c|ccc|ccc|}
\hline $\mathrm{p} / \mathrm{d}$ & $\begin{array}{c}\mathrm{p} \\
(\mu \mathrm{m})\end{array}$ & \multicolumn{3}{|c|}{ SIW } & \multicolumn{3}{c|}{ GAP WAVEGUIDE } \\
\hline & & $\mathrm{S} 11$ & $\mathrm{~S} 21$ & $\alpha_{\text {leakage }}(\mathrm{dB} / \mathrm{m})$ & $\mathrm{S} 11$ & $\mathrm{~S} 21$ & $\alpha_{\text {leakage }}(\mathrm{dB} / \mathrm{m})$ \\
\hline 1.55 & 155 & -35.67 & -0.0012 & 0.009 & -30.78 & -0.28 & 2.57 \\
\hline 2.05 & 205 & -31.14 & -0.0048 & 0.037 & -59.82 & -0.118 & 0.972 \\
\hline 2.45 & 245 & -28.36 & -0.017 & 0.132 & -39.57 & -0.05 & 0.395 \\
\hline 3.1 & 310 & -27.16 & -0.096 & 0.78 & -34.76 & -0.042 & 0.33 \\
\hline 4.1 & 410 & -7.27 & -1.67 & 1.81 & -36.55 & -0.128 & 1.06 \\
\hline
\end{tabular}

The progression of the leakage attenuation for both structures is depicted in Figure 3.8. For SIW, the energy leakage becomes remarkable as the distance 
between vias increases. The gap waveguide exhibits a different behavior. When the relationship p/d is less than 2.5, gap waveguide exhibits higher losses than SIW. However, between $\mathrm{p} / \mathrm{d}=2.5$ and $\mathrm{p} / \mathrm{d}=3$, the leakage losses in the gap waveguide is constant and it exhibits a low leakage losses value. From this point, leakage losses increases being lower than SIW. The interpretation of this behavior of the gap waveguide is due to the stop-band created in this structure. Varying $\mathrm{p}$, the stop-band undergoes range variations. The theoretical computed values for the cut-off frequency of the TE10 propagated mode in SIW and the stopband of the gap waveguide in function of $\mathrm{p} / \mathrm{d}$ are shown in Figure 3.8. Moreover, the minimum and maximum frequency of the stop-band of gap waveguide is represented in Figure 3.9. In the case of SIW, the frequency of interest (170 GHz) is not affected by the variation of $\mathrm{p} / \mathrm{d}$ relationship. Nevertheless, in gap waveguide from $\mathrm{p} / \mathrm{d}$ equal to 3.5 , the maximum frequency of the stop-band is lower than $170 \mathrm{GHz}$; hence losses are not only due to leakage but also to the multimodal configuration because this frequency is outside of the stop-band region.

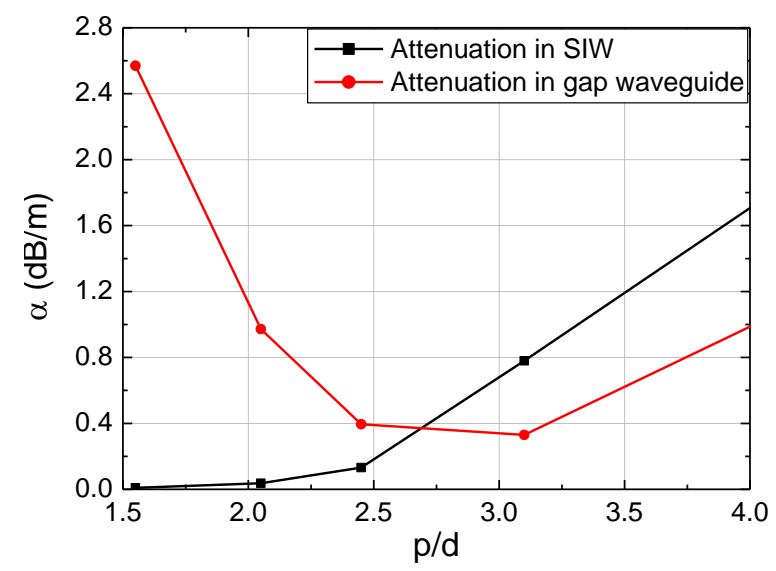

Figure 3.8. Computed leakage attenuation versus p/d.

However, the flexibility in the design of the gap waveguide allows decreasing the gap height to change the stop-band. Therefore, we can reduce leakage losses. In conclusion, this value of $\mathrm{p} / \mathrm{d}$ to avoid leakage losses in gap waveguide is less restrictive because we can change other parameters and have a more flexible choice in dimensions of diameter and periodicity of vias.

Results of the impact of varying $\mathrm{p}$ to enhance the stop-band are reported in Table 3.5. For a $\mathrm{p} / \mathrm{d}=4.1$, the resonance frequency $170 \mathrm{GHz}$ is out of the stop-band 
range, for this reason losses becomes remarkable. In SIW, the cut-off frequency is defined as (3.5).

$$
\mathrm{f}_{\mathrm{c}}=\frac{\mathrm{c}}{2 \sqrt{\varepsilon_{\mathrm{r}}}}\left(\mathrm{w}-\frac{\mathrm{d}^{2}}{0.95 \mathrm{p}}\right)^{-1}
$$

In (7), $\mathrm{c}$ is the speed of light in vacuum, $\varepsilon r$ the relative dielectric permittivity of the substrate, $\mathrm{w}$ the transverse distance between vias, $\mathrm{d}$ the diameter of the metal vias and $p$ their longitudinal spacing. This formula is valid for (3.6).

$$
\mathrm{p}<\frac{\lambda_{0}}{2 \sqrt{\varepsilon_{\mathrm{r}}}} \quad \mathrm{p}<4 \mathrm{~d}
$$

Table 3.5. Impact of p/d over the stop-band of gap waveguide and the fc of SIW (distance $\mathrm{x}=$ wridge $+1.5 \mathrm{sp}$, hgap $=200 \mathrm{um}$ )

\begin{tabular}{|c|c|c|c|c|c|}
\hline \multirow{2}{*}{$\mathbf{p} / \mathbf{d}$} & $\mathbf{p}$ & SIW & \multicolumn{3}{|c|}{ GAP WAVEGUIDE } \\
\cline { 4 - 6 } & \multirow{2}{*}{} & fcTE10 & \multicolumn{3}{|c|}{ Stop-band } \\
\cline { 4 - 6 } & & & fmin (GHz) & fmax(GHz) & Bandwidth \\
\hline 1.55 & 155 & 97.7 & 156 & 194 & 37.6 \\
\hline 2.05 & 205 & 95.2 & 154 & 192 & 37.3 \\
\hline 2.45 & 245 & 93.86 & 153 & 191 & 37.2 \\
\hline 3.1 & 310 & 92.7 & 152 & 181 & 29.4 \\
\hline 4.1 & 410 & 91.5 & 149 & 162 & 12.7 \\
\hline
\end{tabular}

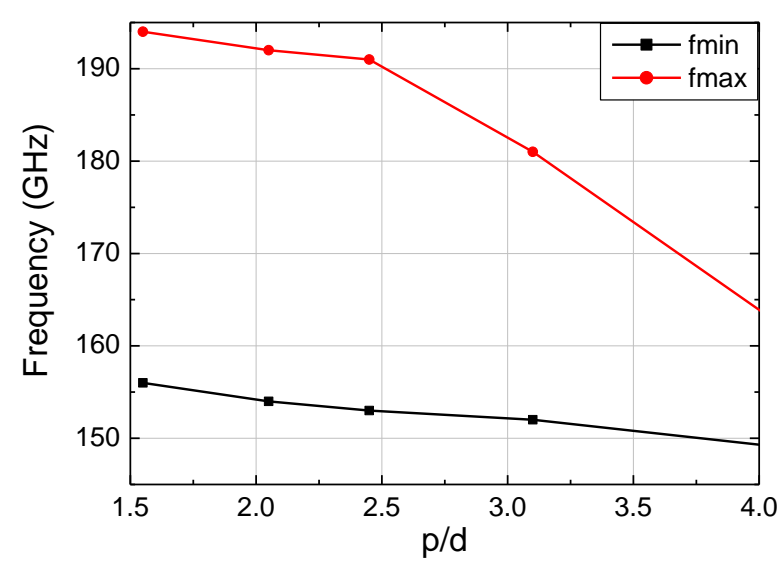

Figure 3.9. Impact of the variation of $p$ over the stopband for the gap waveguide. 
The E-field propagated for the SIW (in the dielectric) and gap waveguide (in the air) is represented in Figure 3.10 at $170 \mathrm{GHz}$. It is observed that for this relationship, $\mathrm{p} / \mathrm{d}=4.1$, leakage loss are very important in SIW structure, because it does not fulfill the condition $\mathrm{p} / \mathrm{d}<2.5$. However, in the gap waveguide, E-field remains confined in the area of interest.

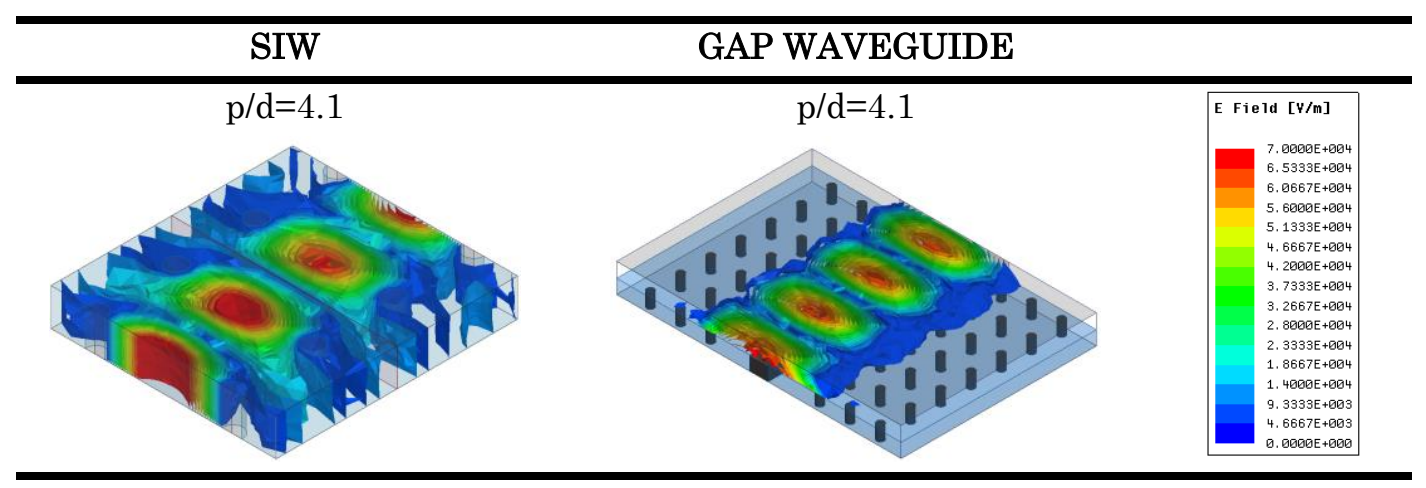

Figure 3.10. E-field distribution in the dielectric for SIW and in the air gap for GW for the relationship $\mathrm{p} / \mathrm{d}=4.1$ (considering no lossy materials).

By looking at the above results, it can be observed how SIW is more sensitive to via spacing changes than via diameter changes. Therefore, gap waveguide offers fewer limitations in the dimensioning of the structures, providing a wider range to the design for high frequency applications.

To characterize the gap waveguide design, the influence of the distance between the ridge and the first row of vias (distance $\mathrm{x}$ ) over the leakage losses is depicted in Figure 3.11. This distance $\mathrm{x}$ is defined by the addition of the half length of the ridge (wridge/2) with several pin periodicity values $(p)$. In this case, the definition of leakage loss is based on the fact that the E-field is not confined between the ridge and the upper metal plate. Varying this distance, we observe some evolution profiles of the $\mathrm{Q}$-factor. Up to wridge $/ 2+2 * \mathrm{p}$, leakage loss decreases as the distance $\mathrm{x}$ increase. From this point, the electromagnetic field is not confined between the ridge and the upper metal plate, it is dispersed between the ridge and the first row of vias. For this reason, leakage loss becomes significant. 


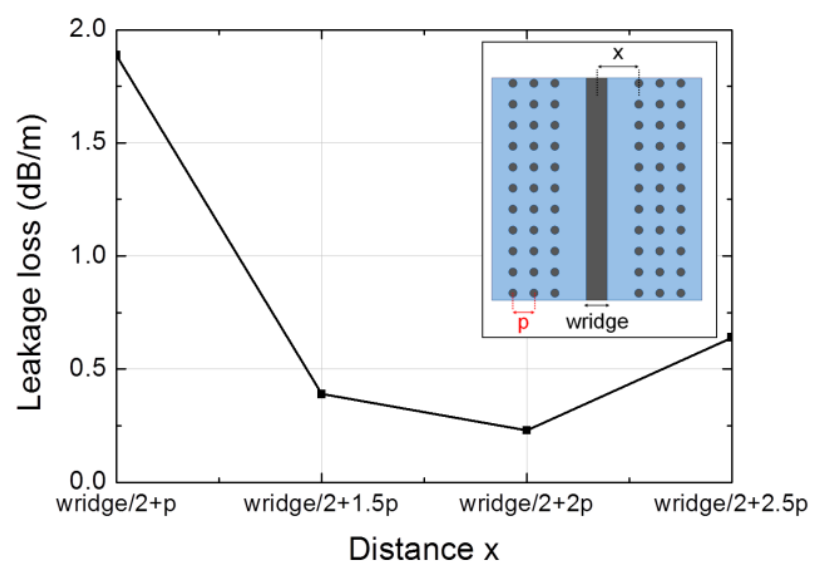

Figure 3.11. Evolution of the leakage losses $(\mathrm{dB} / \mathrm{m})$ varying the distance $\mathrm{x}$.

\subsubsection{Comparison of the E-field distribution in gap waveguide and SIW}

As it has been seen in section 3.3.2, SIW technology is subject to limits more restrictive than gap waveguide. To a simple visualization, Figure 3.12 shows the E-field distribution at the surface of the upper metal plate for some selected values of via periodicity for both the gap waveguide and the SIW configuration.

In SIW structures the electric field is confined and the stop-band appears when the via spacing $(\mathrm{p})$ fulfill the ratio $\mathrm{p} / \mathrm{d}<2.5$. The $\mathrm{E}$-field is represented by increasing $\mathrm{p}$ while keeping the other dimensions unchanged $(\mathrm{d}=0.1 \mathrm{~mm}$ and total length $=2.5 \mathrm{~mm}$ ). From $\mathrm{p} / \mathrm{d}=2.5$ condition, leakage loss occurs in the sidewalls of the SIW. In contrast, the Gap Waveguide configuration remains more tolerant to wider pin spacing. Furthermore, the propagation in the gap waveguide takes place mostly in the air gap between the strip and the upper plate and not in the dielectric as is the case for SIW, thus reducing the propagation losses. 


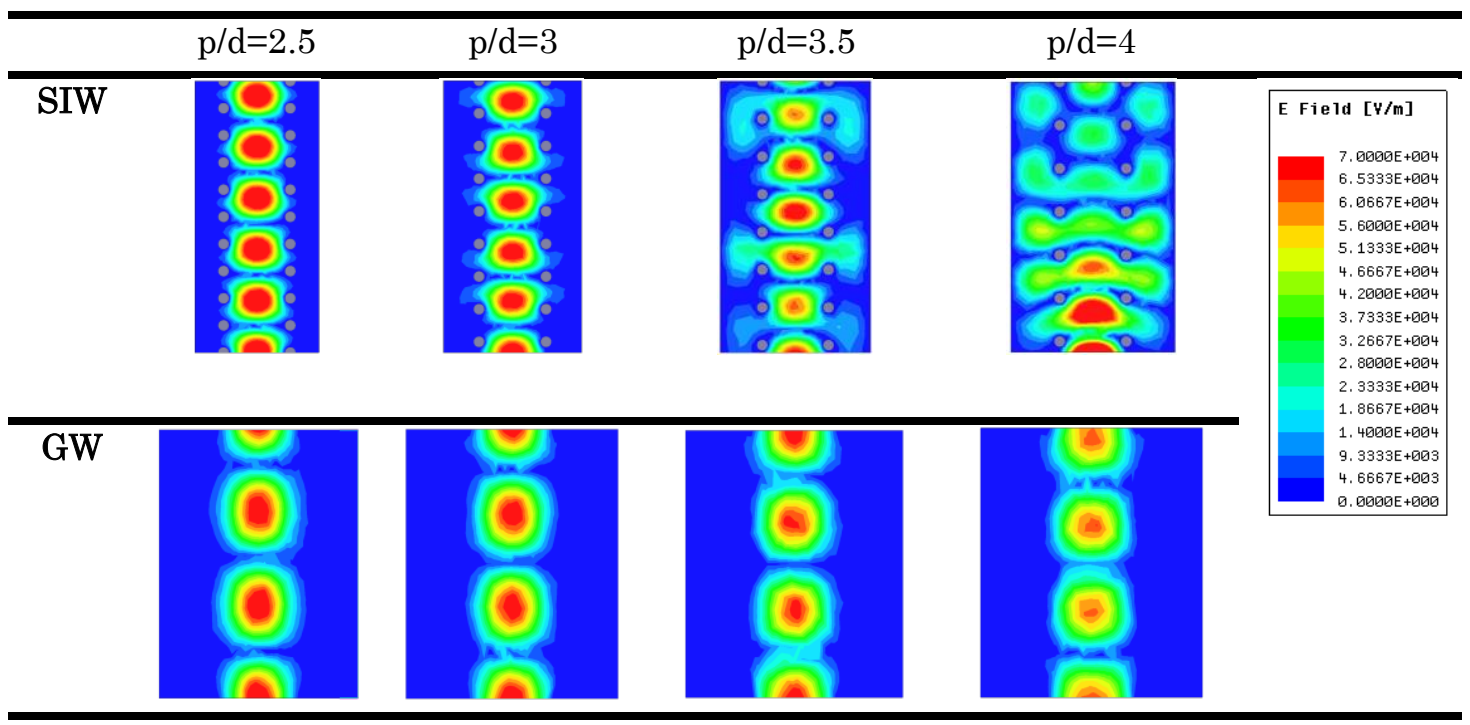

Figure 3.12. Vertical E-field distribution at the surface of the upper metal plate.

\subsubsection{Region of interest}

In SIW technology, the region of interest has been presented in [62] and a formula for leakage losses in [67]. The bibliography proposes numerical studies, mainly based on the variation of geometrical parameters as vias diameter, $d$, or periodicity, p, which are the mainly causes of leakage losses in SIW. We can summarize the geometrical limits for SIW by these equations:

$$
\begin{array}{lll}
\frac{\mathrm{p}}{\mathrm{d}}>1 & \mathrm{p}<\frac{\lambda_{\mathrm{c}}}{4} \\
\frac{\mathrm{p}}{\mathrm{d}}<2.5 & \mathrm{p}>\frac{\lambda_{\mathrm{c}}}{20}
\end{array}
$$

On the other hand, in previous section of this chapter, computed leakage losses in terms of the geometrical parameters $d$ and $p$ has been presented for the gap waveguide. With these results, we can define the leakage region for the gap waveguide technology. The limit to avoid leakage losses as it has been seen is around $\mathrm{p} / \mathrm{d}=3$. For higher ratios, attenuation due to leakage increases. However, other geometrical parameters could impact and improve the limit to avoid leakage losses as for example the gap height. These leakage losses in gap waveguide are due not only to the gaps between vias, but the modification of $p$ and $d$ has an impact in the stop-band created by the HIS. Therefore, these leakage losses are also due to the dispersion because and we cannot be in the mono-modal band, but is in the multi-modal band. 


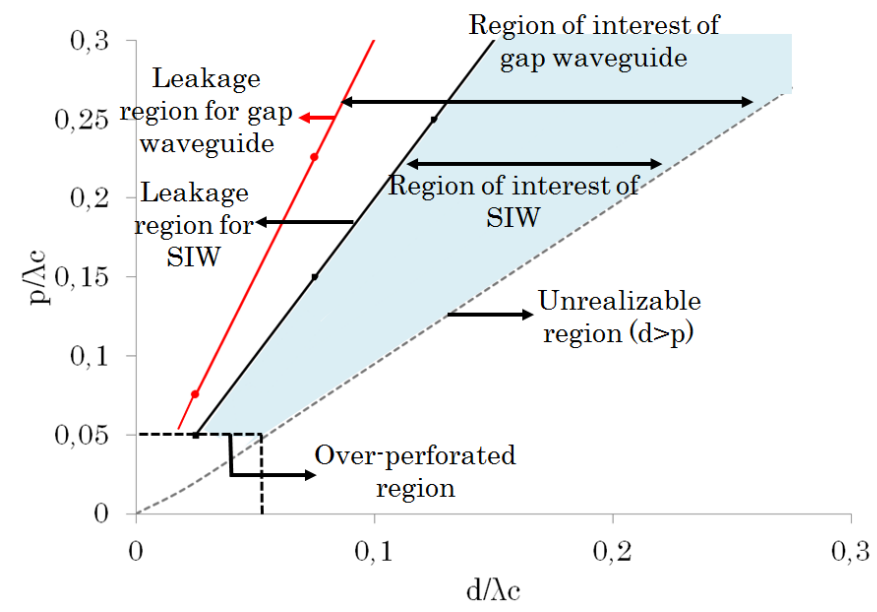

Figure 3.13. Region of interest for the SIW and gap waveguide in the plane of $d / \lambda c, p / \lambda c$.

As it is seen in Figure 3.13, choice of the via diameter and the periodicity between vias in gap waveguide is less restrictive than in SIW. In the millimetrewave range, the choice of these parameters is restrictive as it can be compatible with the fabrication process and not always it is allowed small diameter or a narrow distance between vias. In this context, gap waveguide is presented as an alternative technology for millimetre-wave devices, reducing the constraints in terms of design for specific values of diameter and distance between vias.

\subsection{Conductor and dielectric losses}

Both $3 \mathrm{Ag} / 2$ length resonators are designed to observe a third order resonance to have an adequate length with the aim to analyze the impact of the via periodicity in the losses. In the case of SIW, the length of the resonators must correspond with the value $1.5 \mathrm{Ag}$. This value is $1.09 \mathrm{~mm}$ (considering the resonance frequency at $170 \mathrm{GHz}$ ). In the gap waveguide structure the length of the structure should be $1.5 \Lambda_{0}$, being $2.65 \mathrm{~mm}$.

Both resonators based on SIW and gap waveguide technology are shown in Figure 3.14. All materials considered in the models are lossy. For the conductor, gold has been chosen and the dielectric is Ferro A6-M LTCC tape. 


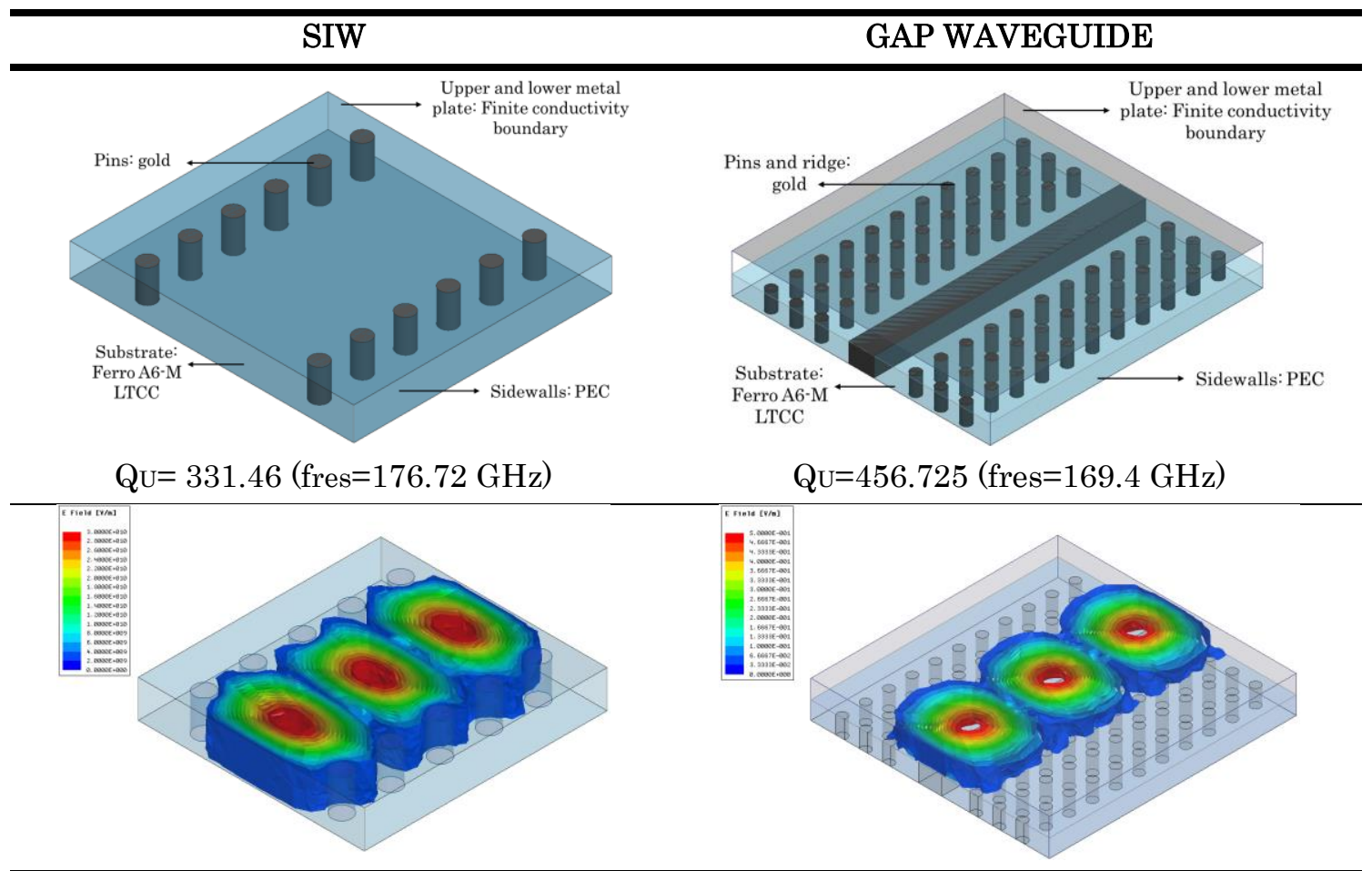

Figure 3.14. Design and computed Q-factor for both configurations.

As it has been explained in the beginning of this section, there are basically three important mechanisms of losses that involve gap waveguide and SIW. Once, leakage losses have been analyzed, a study of loss in dielectrics and conductors is presented.

The variation of the conductor and dielectric loss versus $d$ is limited, as discussed in [68]. In particular, the conductor loss slightly decreases when increasing the diameter $\mathrm{d}$ of the vias.

In [69] it is shown that the conductor loss are small when the metal vias are large and closely spaced. The physical explanation of this effect is that, when vias are close to each other and wide, the vertical electric current density on the metal vias flows on a wider surface, thus reducing its amplitude.

For the analysis of the dielectric loss, the approach is based on the $\mathrm{Q}$-factor value obtained by means of the Eigenmode solver of HFSS.

Let us consider the resonators described in previous section and fix $\mathrm{p} / \mathrm{d}=2.5$ for $\mathrm{SIW}$, and the distance $\mathrm{x}=\mathrm{W}_{\text {ridge }} / 2+1.5 \mathrm{p}$ for gap waveguide (total gap waveguide length is $2.65 \mathrm{~mm}$ ). We compute the $\mathrm{Q}$-factor due to the conductor and dielectric 
loss. Conductor material is gold (conductivity $\sigma=41 \mathrm{GS} / \mathrm{m}$ and relative permeability $\mu \mathrm{r}=0.99996)$ and dielectric Ferro A6-M LTCC tape.

Firstly, we analyze the impact of the dielectric losses. For this reason, for the computed analysis we use perfect electric conductor condition for all conductors including upper and lower metal plates. Material properties are represented in Figure 3.15.

GAP WAVEGUIDE

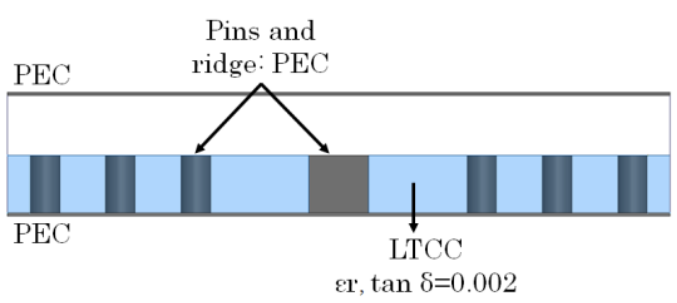

SIW

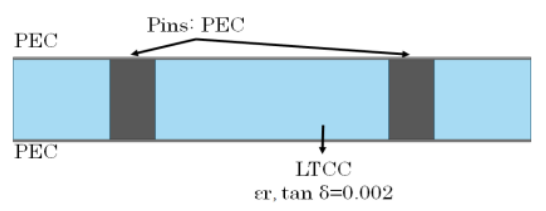

Figure 3.15. Properties of the materials used in Simulations: Ideal conductor and lossy dielectric.

Table 3.6. Computed Q-factor due to the dielectric loss (no conductor losses are considered).

\begin{tabular}{|cccc|}
\hline & $\mathrm{Q}$ & $\mathrm{f}$ & Dielectric losses $(\mathrm{dB} / \mathrm{m})$ \\
\hline Gap waveguide & 970.89 & 169.5 & 4.45 \\
\hline SIW & 500.01 & 176.8 & NOT quasi-TEM \\
\hline
\end{tabular}

In the next case, we consider losses in the conductor, upper and lower metal plates and vias. No losses in the dielectrics are considered. With this approach, we can compute losses due to conductor in both structures (Figure 3.16).

GAP WAVEGUIDE

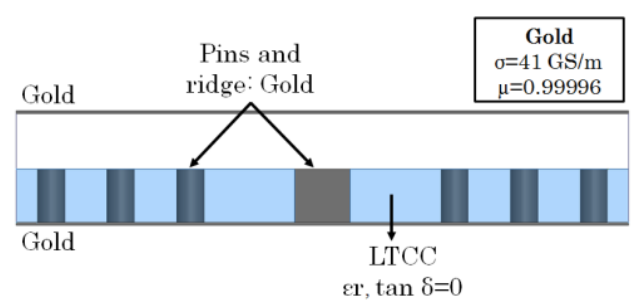

SIW

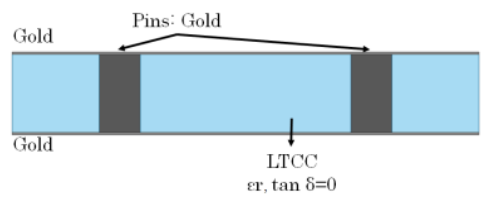

Figure 3.16. Properties of the materials used in simulations: Ideal dielectric and lossy conductors. 
Table 3.7. Computed Q-factor due to the conductor loss (no dielectric losses are considered).

\begin{tabular}{|cccc|}
\hline & Q & f & Conductor losses $(\mathrm{dB} / \mathrm{m})$ \\
\hline Gap waveguide & 729.68 & 169.41 & 5.92 \\
\hline SIW & 832.57 & 176.7 & NOT quasi-TEM \\
\hline
\end{tabular}

To characterize the impact of the metallic vias in the total of the conductor loss, now we compare losses when only vias are lossless.

GAP WAVEGUIDE

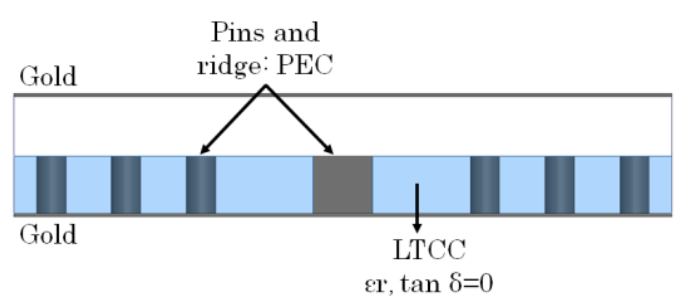

SIW

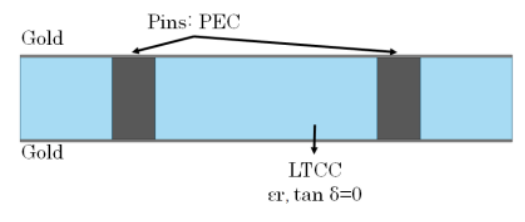

Figure 3.17. Properties of the materials: Ideal dielectric, ideal vias metallization (PEC) and losses in the upper and lower metal plate.

Table 3.8. Conductor loss characterization: Gold for upper and lower metal plate, PEC for vias.

\begin{tabular}{|cccc|}
\hline & Q & f & Conductor losses in vias $(\mathrm{dB} / \mathrm{m})$ \\
\hline Gap waveguide & 1939.13 & 169.5 & 2.23 \\
\hline SIW & 1208.95 & 176.72 & NOT quasi-TEM \\
\hline
\end{tabular}

In Figure 3.18, Q-factor due to conductor losses is represented for gap waveguide and SIW. Two cases are depicted. Firstly considering conductor losses in plates and vias (Figure 3.16) and secondly with lossless vias and lossy plates (Figure 3.17). Results show that the impact of conductor loss in vias is more important in the gap waveguide structure than in SIW. 


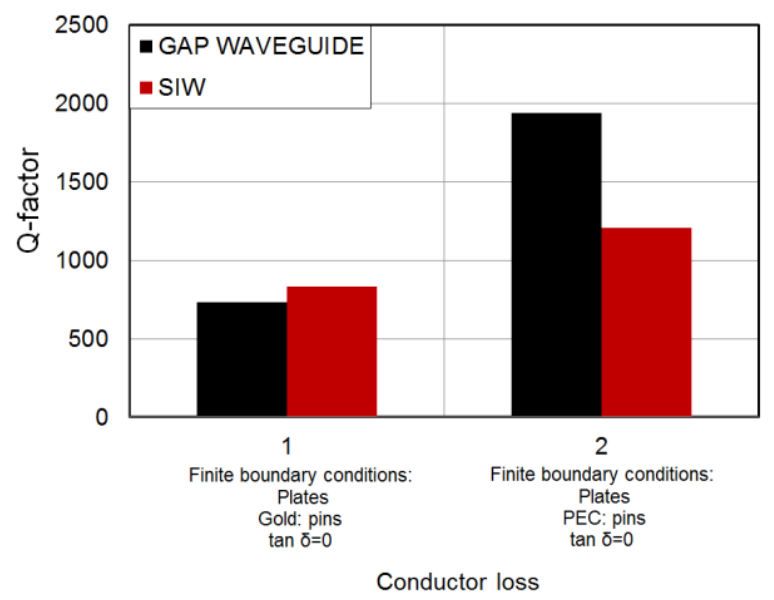

Figure 3.18. Case 1: with lossy vias and plates; Case 2:

With conductor loss in plates and vias without loss.

In Figure 3.19, the results of the analysis considered in the three cases are summarized: 1 and 2 are those cases shown in Figure 3.18 to characterize conductor loss. Case 3 shows the influence of the dielectric loss (analysis performed without conductor loss).

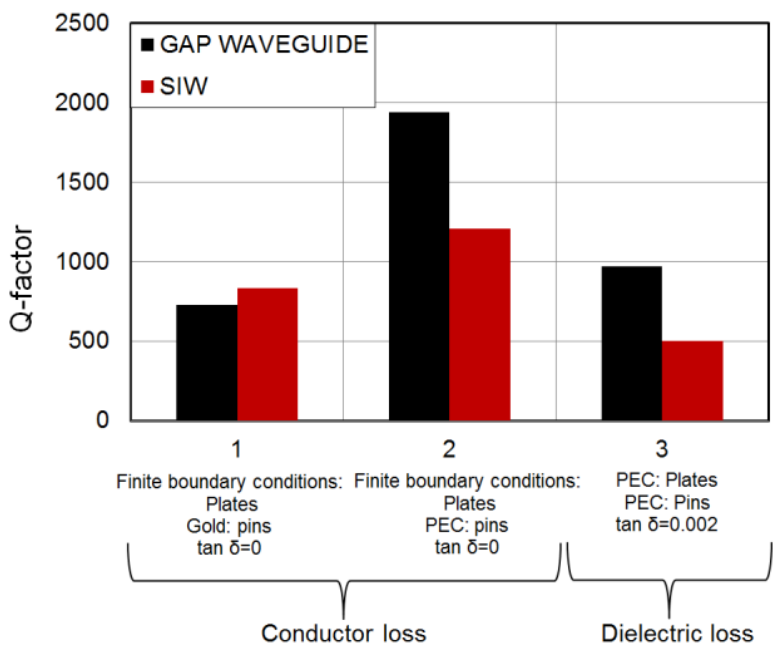

Figure 3.19. Q-factor obtained with the Eigenmode of HFSS considering dielectric, conductor, both or any losses.

Results of this comparison reveals that gap waveguide, for a relationship $\mathrm{p} / \mathrm{d}=2.5$, offers a high $\mathrm{Q}$-factor due to dielectric losses, and lower due to conductor loss in comparison with SIW. In SIW conductor losses are more important. To 
reduce conductor losses in SIW, it is necessary to increase the thickness of the substrate h.

\subsection{Conclusion}

In this chapter, a comparison between gap waveguide and SIW in terms of losses has been presented.

Initially, the methodology employed for the losses study has been explained. This procedure consists of an analysis of the transmission parameters for the leakage loss characterization and an analysis of the Eigenmode properties creating resonators based on both technologies for obtaining the Q-factor that allows characterizing the attenuation constant.

Secondly, the design of a gap waveguide and SIW structure operating at 170 $\mathrm{GHz}$ has been presented. The design limitations in terms of via diameter and periodicity between vias were applied.

Then, leakage loss study was presented. Analysis of the region of interest and data values determined that gap waveguide is more flexible in terms of design parameters to avoid leakage losses between the gaps of the metal vias than SIW.

Finally, conductor and dielectric loss are computed by the analysis of the $\mathrm{Q}^{-}$ factor of both resonators, based on gap waveguide and SIW technology. Two conclusions are worth mentioning: 1) the impact of dielectric loss is more important in SIW than in gap waveguide due to the propagation in the dielectric. 2) Conductor loss is a little more significant in gap waveguide than in SIW, especially in the metal vias.

In summary, in this chapter losses in gap waveguide and SIW are compared aiming at characterizing both structures for the $\mathrm{mm}$-wave range. Gap waveguide overcomes some technological limitations of SIW in terms of leakage loss and dielectric loss. In terms of conductor loss, SIW exhibits a higher Q-factor. The choice of the structure depends on the application and the manufacturing technique limitations. 


\section{Chapter 4}

\section{Gap Waveguide using PCB and LTCC Technology}

\subsection{Introduction}

This chapter aims to demonstrate the performance of gap waveguide structures based upon PCB and LTCC technologies. The design of gap waveguide with PCB and LTCC has been presented in Chapter 2. Therefore, in this chapter, a feeding transition is designed in order to validate the manufactured gap waveguide. Initially, design and manufacturing of the structure have been done using PCB techniques, particularly on RO4003 substrate. Manufacturing and measurements of such structure will be analyzed. Later, LTCC process will be explained with the aim of understanding the most critical parts of this manufacturing process. One of these parts is to make cavities, corresponding to the air gap of the gap waveguide design. With this purpose, a study of the manufacturing process of cavities in LTCC has been done and will be presented. The manufacturing of external and internal cavities will be illustrated. Cavity shrinkage and 
deformation as a result of the process will be discussed. Finally, the manufactured gap waveguide structure and measurements will be presented. Technological problems related to this manufacturing will also be addressed.

\subsection{Final prototype}

In this section, the complete breadboard including the feeding transition is presented. The structure has been modeled to operate at $60 \mathrm{GHz}$. The complete breadboard of the structure is depicted in Figure 4.2. As it can be seen, the structure is composed basically by three parts: the feeding transition, the cavity to create the air gap and the surface with vias and the transmission line forming the gap waveguide.

The feeding transition to the gap waveguide involves the design of the transition in two steps. The first one consists of a coplanar to microstrip transition and the second, a microstip to gap waveguide transition with an $\mathrm{H}^{-}$ shape slot in the ground plane of the microstrip. The ground plane of the microstrip contains the $\mathrm{H}$-shape slot apertures for the energy coupling. The $\mathrm{H}^{-}$ shape aperture reduces the back radiation of the rectangular slot and also the length compared with a conventional rectangular aperture [70]. The characteristic impedance of the microstrip line is fixed to $50 \Omega$. In the coplanar transition the center signal contact establish direct connection with the microstrip line and the outer probes are connected to ground planes.

Then, a cavity is necessary to create the air zone where the propagation will take place. According to the manufacturing technique, some possibilities to create this cavity are available. For example with multilayer PCB technology, using laser cutting, we can create different shapes forming the cavity by cutting each PCB and then stacking them all. In the case of LTCC or HTCC, a cavity can be created in one co-firing process for all the structure. The creation of LTCC cavities will be explained with more detail in 4.5.2.

Finally, we can see the surface of vias (or mushroom-shape) embedded in a substrate to create the HIS condition and the transmission line to propagate the E-field. Top view shows the mushroom surface and the transmission line while bottom view depicts the ground plane. 


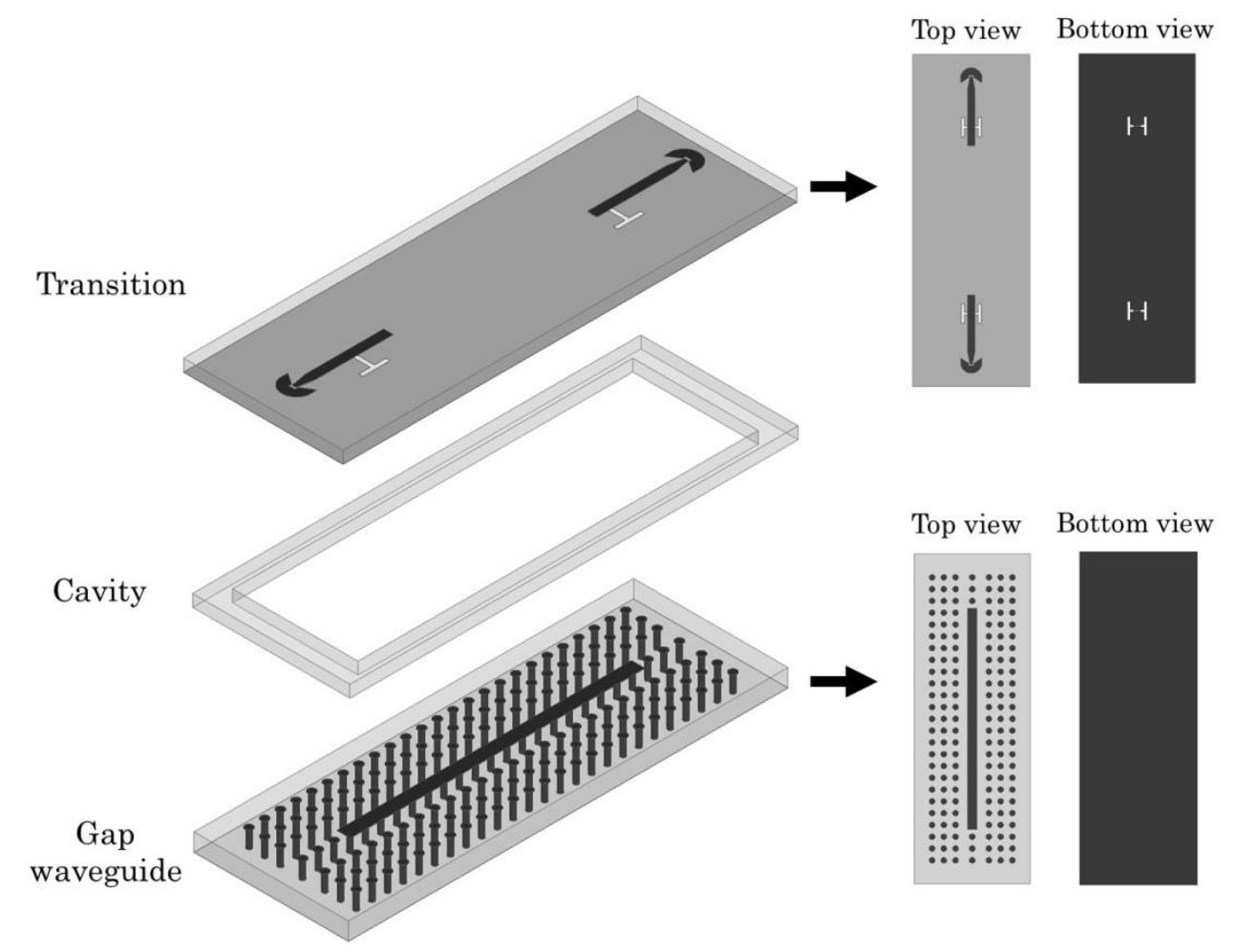

Figure 4.2 Breadboard of the complete structure: Coplanar to microstrip to H-shape coupled aperture transition, cavity layer to create the air gap and gap waveguide including the surface of vias embedded in a substrate and transmission line.

\subsection{PCB and LTCC manufacturing techniques}

Once the final structure has been presented, the advantages and the drawbacks of Low Temperature Co-fired Ceramic manufacturing technique are discussed in this section. Commonly, the simplest and lowest-cost technique for the manufacturing of high frequency devices is the Printed Circuit Board techniques. Therefore, multilayer PCB techniques have been presented in the literature to integrate as in our case several modules in only one prototype. These layers are stacked together, however this process increase the cost and the production cycle. Moreover, for the creation of cavities, the stacking method can increase the thickness of the cavity that can produce a change in the impedance value that can produce a mismatching of the structure behavior.

Multilayer PCB is presented as a candidate for bio-chemical analysis due to the versatility, small sample, portability and rapid processing. To take advantage of this technology, typically, these devices are manufactured with multiple 
components and functions to be integrated in a single platform. However, the main drawback of PCB technology for microfluidic applications is the nonbiocompatibility of materials.

LTCC technology is presented as a good alternative to manufacturing our gap waveguide structure because it allows manufacturing the three parts described in Figure 4.2 in one step. Nevertheless, some critical points of the LTCC process should be analyzed, such as:

a) Via filling

b) Alignment

c) Cavity creation

a) First, we focus on the problem related to the metallization of the vias. Our via filling process with a thick film conductor to ensure the electrical connection between layers uses the same technique as employed for screen printing. We can observe two kinds of difficulties: misalignment of the filling and control of the quantity of gold paste applied to vias. We have tested both problematics as we can see in Figure 4.3 and Figure 4.4.
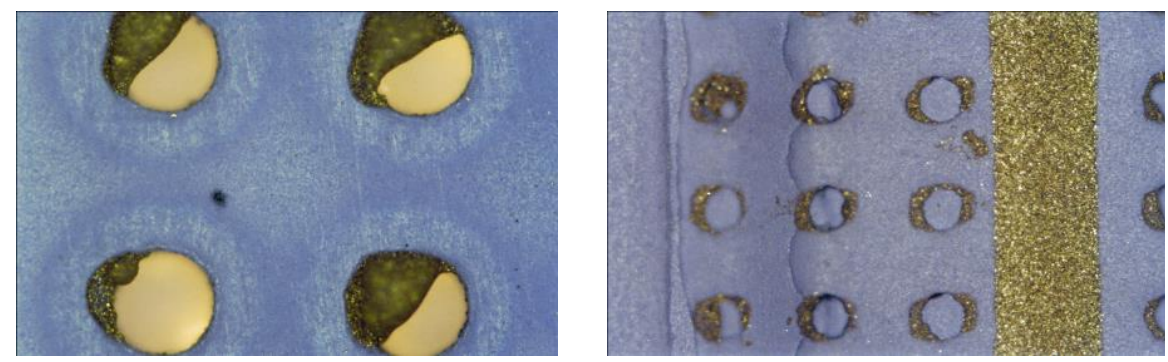

Figure 4.3. Difficulties in the via filling after co-firing.
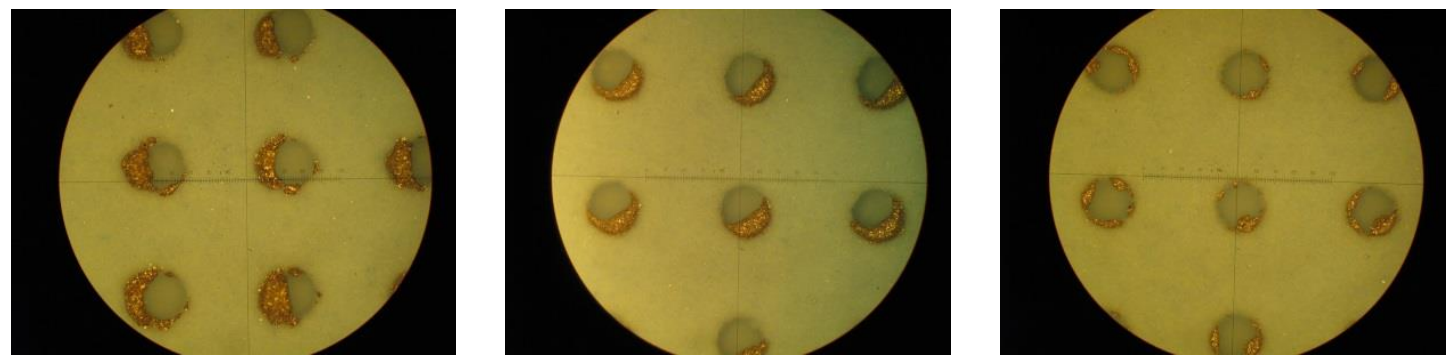

Figure 4.4. Via filling difficulties: Pictures 1 and 2 from left to right: Shift in the via filling due to the misalignment in the thick film screen printer. Picture 3 from left to right: insufficient paste quantity applied to the vias. 
The misalignment in the via fill process is due to the bad screen-to-substrate alignment. In fact, with the optical camera integrated in our via filling system, there is a small gap where a shift in the alignment can occur. Typically the misalignment value produced is around $100 \mu \mathrm{m}$.

b) Next critical point is the alignment of stacked layers as it is shown in Figure 4.5. This misalignment can be due to the uniaxial method for the lamination process, which can cause difficulties in establishing perfect parallel lamination and create variability in the pressure and in temperature.

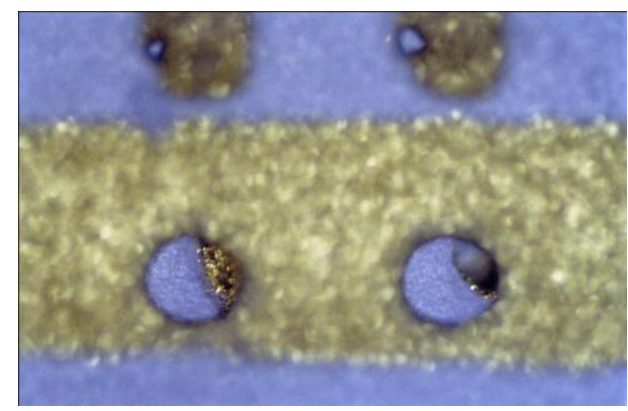

Figure 4.5. Misalignment due to lamination process.

c) Finally, a crucial point for the LTCC process is the creation of cavities, especially in the case of internal cavities. A further study and manufacturing of external and internal cavities has been done in this thesis and will be shown in Section 4.5.2.

In this chapter, we aims to validate the gap waveguide design with PCB and LTCC manufacturing techniques. First, the gap waveguide has been modeled and manufactured with RO4003.

\subsection{Gap waveguide design using PCB multilayer technique}

The design of gap waveguide with PCB at high frequency has been validated in past publications [71][72] and also considerations designs have been presented in Chapter 2 (Section 2.2) of this manuscript. Geometrical parameters for the design of the gap waveguide with PCB are shown in Table 4.1. 
Table 4.1. Dimensions of the gap waveguide design using RO4003 substrate.

\begin{tabular}{|ccc|}
\hline \multicolumn{3}{|c|}{ Geometrical dimensions for the gap waveguide } \\
\hline Pin diameter & $\mathrm{d}$ & $200 \mu \mathrm{m}$ \\
\hline Patch diameter & $\mathrm{d} 2$ & $400 \mu \mathrm{m}$ \\
\hline Spacing of the pins & $\mathrm{p}$ & $800 \mu \mathrm{m}$ \\
\hline Dielectric & $\mathrm{RO} 4003$ & $\begin{array}{c}\varepsilon \mathrm{r}=3.55 \\
\text { tan } \delta=0.0027\end{array}$ \\
& & Substrate thickness $=813 \mu \mathrm{m}$ \\
\hline Pin height & $\mathrm{hp}$ & $813 \mu \mathrm{m}$ \\
\hline Width of the strip & wridge & $500 \mu \mathrm{m}$ \\
\hline Air gap height & hgap & $200 \mu \mathrm{m}$ \\
\hline
\end{tabular}

In order to have information about the bandwidth of the structure, the dispersion diagram has been calculated. The stop-band created by the HIS is [40.7-74.8] GHz. Only the quasi-TEM mode is propagated thanks to the presence of the strip, as illustrated in Figure 4.6.

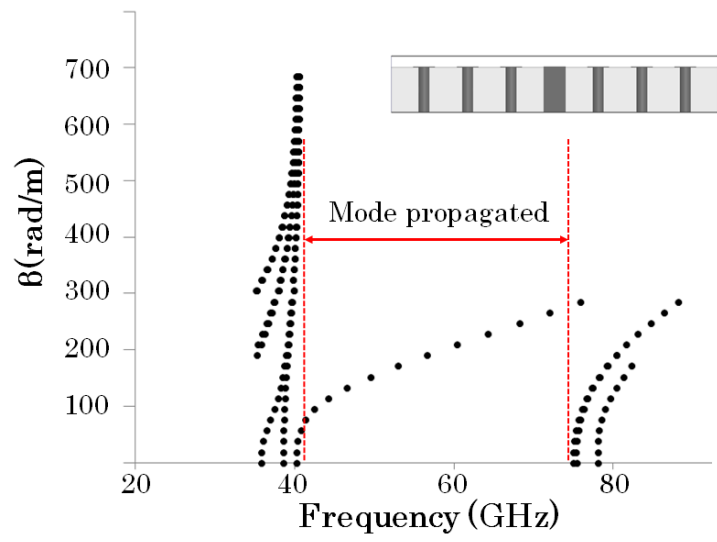

Figure 4.6. Dispersion diagram of the gap waveguide with RO4003.

The computed S-parameters of the gap waveguide design with R04003 are shown in Figure 4.7-a. In this case, all losses in materials are considered namely pins, ground planes and printed lines with copper. RO4003 substrate $(\varepsilon r=3.55$, $\tan \delta=0.0027)$ is used. The gap waveguide is excited by waveports outlined on rectangular surfaces at both ends of the transmission line ridge (Figure 4.7-b). The appropriate width of the waveguide port for the gap waveguide excitation is defined in [73]. 
As it can be seen in Figure 4.7-a, $0.3748 \mathrm{~dB}$ insertion loss at $55 \mathrm{GHz}$ and the return losses exhibits a value lower than $-20 \mathrm{~dB}$ in all the band of interest.

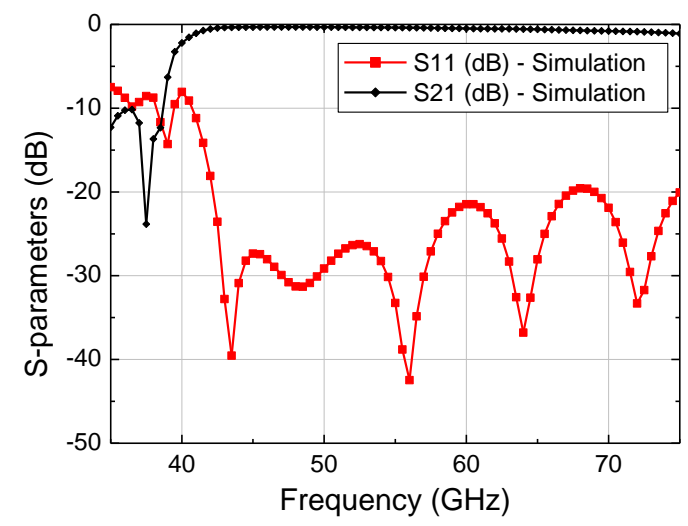

(a)

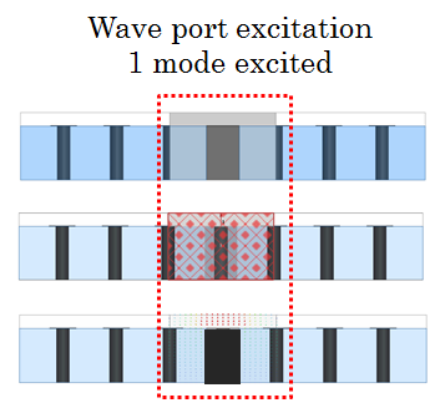

(b)

Figure 4.7. Gap waveguide with RO4003 substrate. a) Transmission and reflection coefficients b) Wave port excitation in HFSS.

The gap waveguide structure being validated, next part deals with the study of the transition to feed the gap waveguide. The proposed transition consists of an aperture-coupled microstrip-to-gap waveguide transition. The aperture is etched in the microstrip circuit ground plane. The microstrip was chosen to have a $50 \Omega$ characteristic impedance. Front view and dimensions of the aperture coupled microstrip-to-gap waveguide transition are depicted in Figure 4.8. The side view of the transition and dimensions between the strip of the microstrip transition and the $\mathrm{H}$-shape aperture are shown in Figure 4.9 and Figure 4.10, respectively.

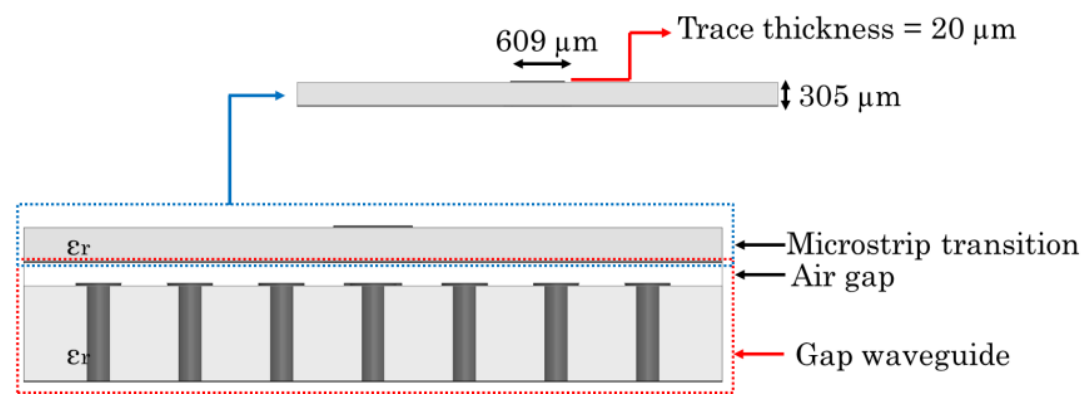

Figure 4.8. Front view and dimensions of the aperture coupled microstrip to gap waveguide transition. 


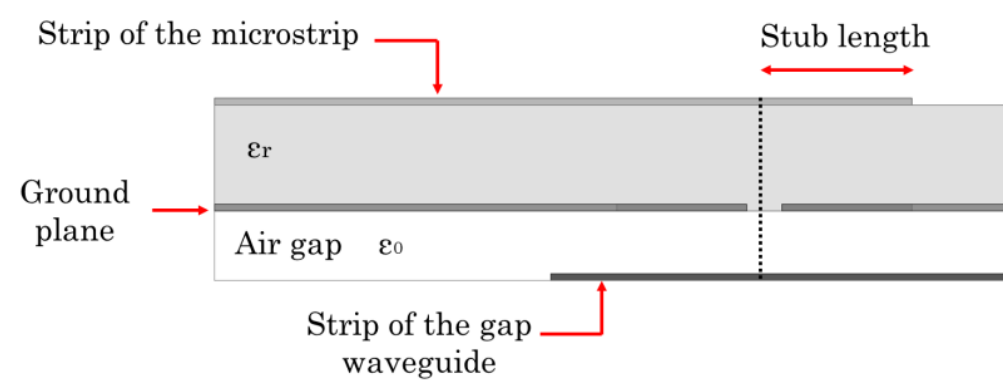

Figure 4.9. Side view of the microstrip to gap waveguide transition.

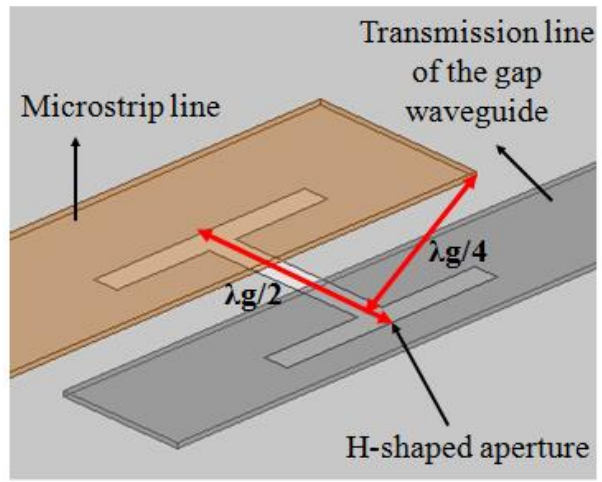

Figure 4.10. H-shape aperture microstrip to gap waveguide transition.

The simulated structure and the E-field distribution in the wave port excitations are depicted in Figure 4.11. Computed results of the back-to-back microstrip to gap waveguide transition are presented in Figure 4.12. Results at $55 \mathrm{GHz}$ show a $-9.8 \mathrm{~dB}$ insertion loss and $-0.66 \mathrm{~dB}$ return loss.

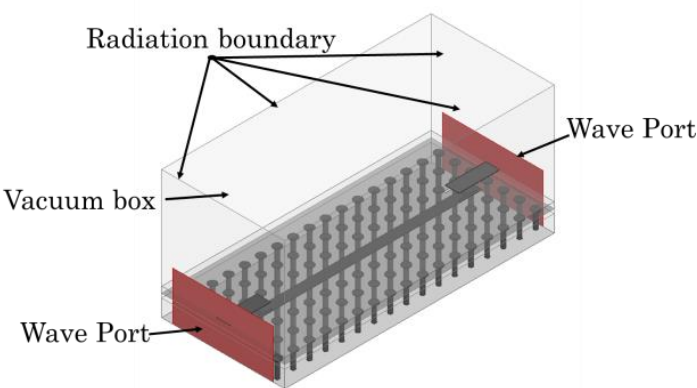

a)

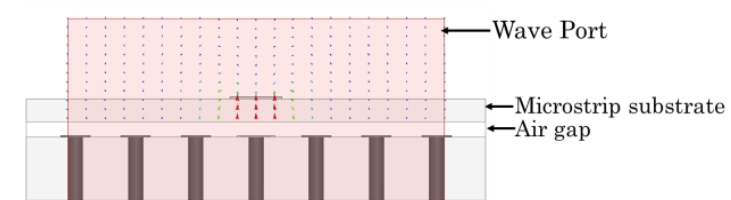

b)

Figure 4.11. Microstrip-to-gap waveguide back-to-back transition: a) 3D view of the structure with HFSS, b) E-field distribution in the wave port excitation. 


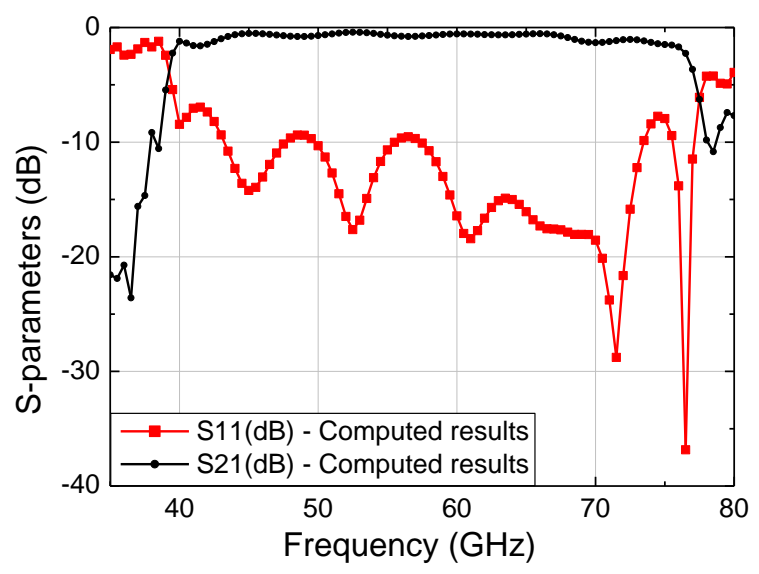

Figure 4.12. Computed results for the transmission and reflection coefficients of the microstrip to gap waveguide transition.

The conductor and dielectric media in the simulation were assumed lossless; therefore all losses can be ascribed to leakywave. We can compute the efficiency of the transition by means of Formula (4.1), which is also represented in Figure 4.13 as a function of the frequency. The H-shape aperture presents a good efficiency and energy coupling between the microstrip and the gap waveguide. Design of the aperture has been optimized to minimize the leakywaves.

$$
\eta=\frac{\left|S_{21}\right|^{2}}{1-\left|S_{11}\right|^{2}}
$$

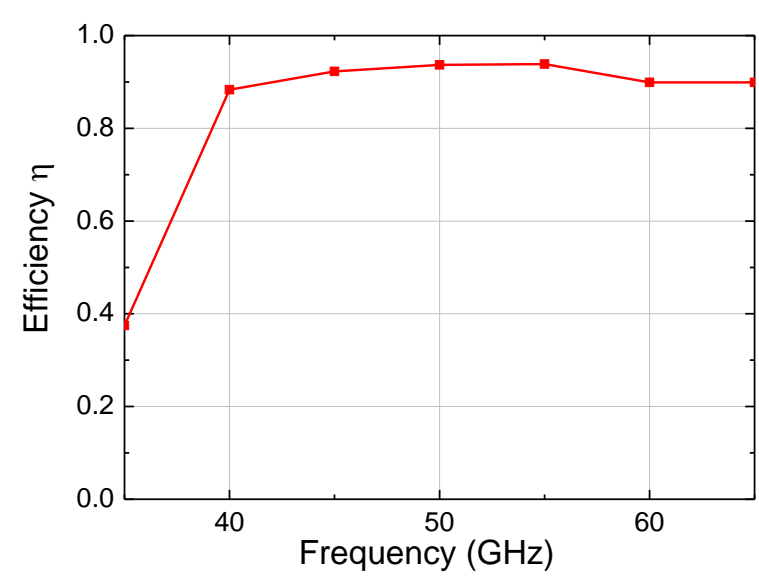

Figure 4.13. Efficiency of the aperture in terms of radiation loss.

Once the microstrip to gap waveguide transition has been designed and validated through simulation, the measurements have been performed using 
coplanar probes. Dimensions of the coplanar-to-microstrip transition are depicted in Figure 4.14.

The performance of the back-to-back transition was simulated and optimized by full wave simulations using HFSS solver. The coplanar waveguide is excited by means of lumped port defined on HFSS and with $50 \Omega$ as reference characteristic impedance. The simulated S-parameters are depicted on Figure 4.15 and show good performances of the proposed structure; the insertion loss are $1.29 \mathrm{~dB}$ and $1.83 \mathrm{~dB}$ (without conductor and dielectric losses and with losses, respectively) at the center operating frequency.

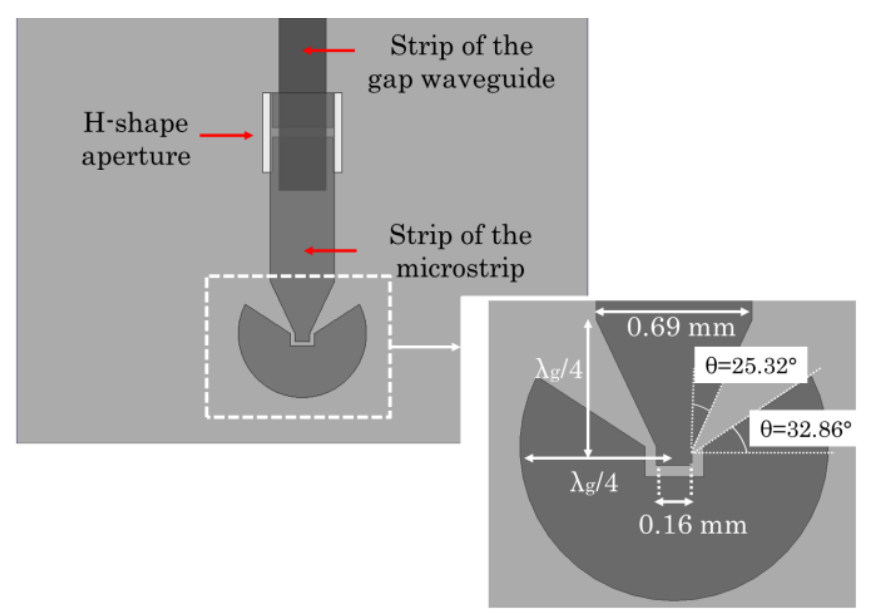

Figure 4.14. Dimensions of the coplanar probe.
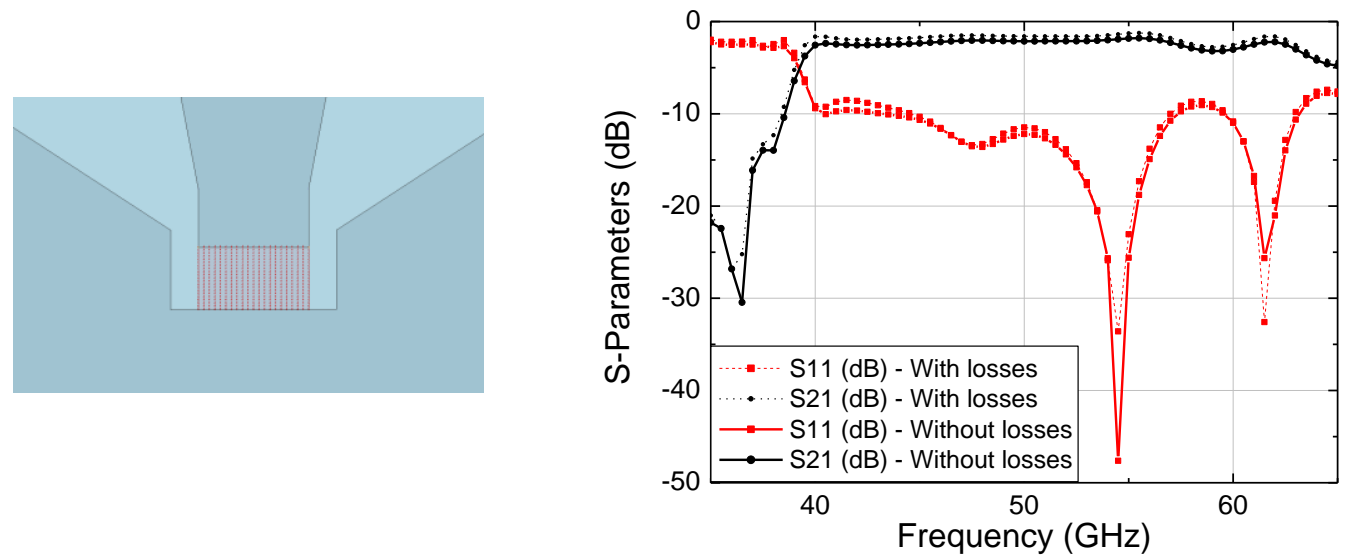

Figure 4.15. Computed results of the back-to-back CPW-to-Microstrip-to Gap Waveguide transition: a) E-field distribution at the Wave Port, b) Computed Sparameters $(\mathrm{dB})$ 


\subsubsection{Manufactured prototype with PCB}

The assembly of the complete design is composed of three separated parts. Firstly, the gap waveguide structure containing the metallic pins, the metallic pads and the transmission line was manufactured. Metallization has been done with copper and the dielectric employed is RO4003. Secondly, the transition explained in Section 4.1.3 has been manufactured, using also copper for the metallization and RO4003 as substrate. Finally, the air gap is created by means of a RO4003 layer forming the air cavity.

The final prototype includes the three parts of our prototype as shown in Figure 4.17: the gap waveguide structure, the transition and the air gap created with a RO4003 layer. In Figure 4.16-a, a picture with the introduction of the support creating the air gap cavity is shown. The three parts are placed in the base with the three alignment pins (Figure 4.16-b) for stacking all the parts with a thick plastic film.

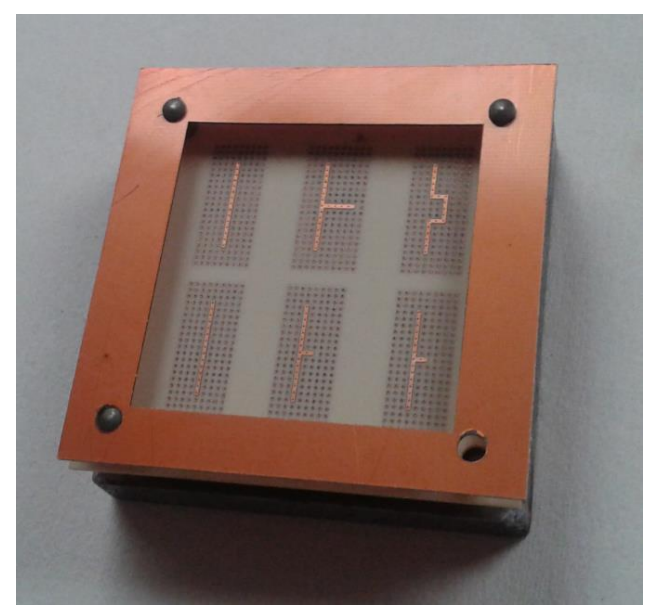

a)

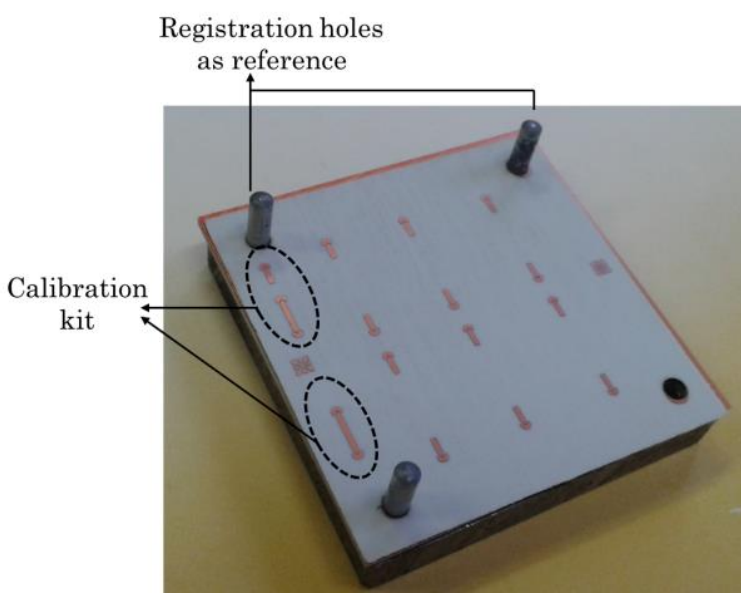

b)

Figure 4.16. a) Picture with the addition of the RO4003 layer to create the air gap cavity; b) Three parts (gap waveguide, transition and layer for the air cavity) before stacking. 


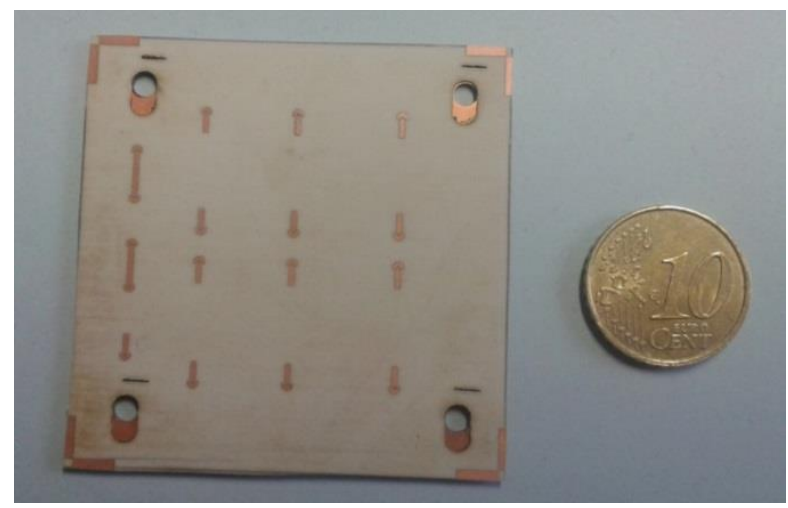

Figure 4.17. Final prototype including the transition and the gap waveguide.

\subsubsection{Gap waveguide prototypes}

The manufactured prototype contains six models based on the gap waveguide concept (Figure 4.18). Prototypes 1 and 2 have the dimensions described in Section 4.1.1 and 4.1.3. Prototypes 3, 4 and 6 are resonators based on gap waveguide technology and they will be analyzed in detail in Chapter 5. Prototype 5 consists of a two $90^{\circ}$ bends in the transmission line forming the gap waveguide.
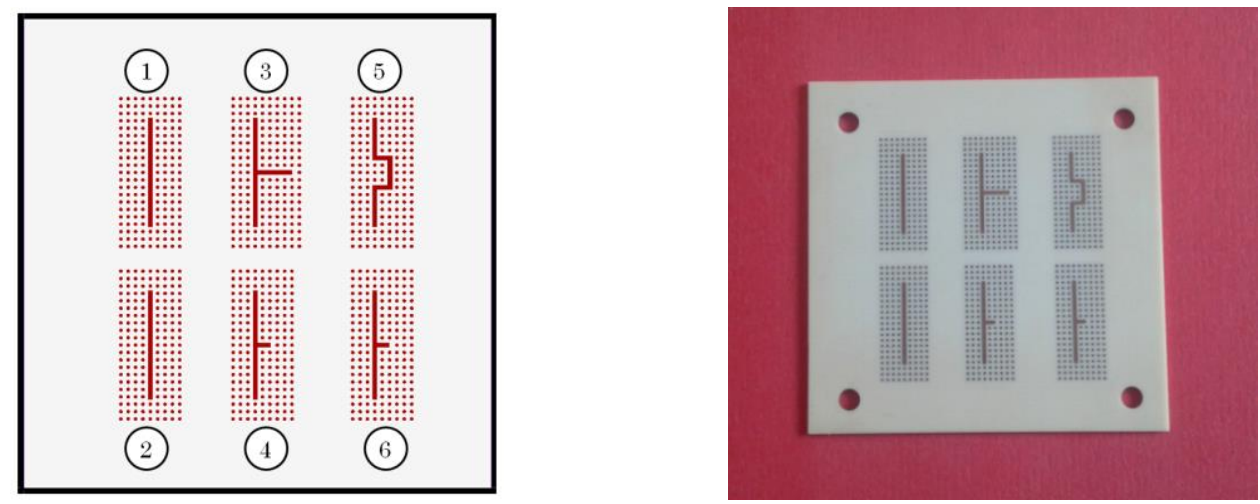

Figure 4.18. a) Layout of the six prototypes with ADS software; b) Manufactured device with RO4003 substrate.

\subsubsection{Transition CPW-to-microstrip-to-gap waveguide}

The transition coplanar-microstrip with $\mathrm{H}$-shape aperture coupled has been presented in Section 4.1.3. The transition has been manufactured using RO4003 substrate, with copper metallization (Figure 4.19). 


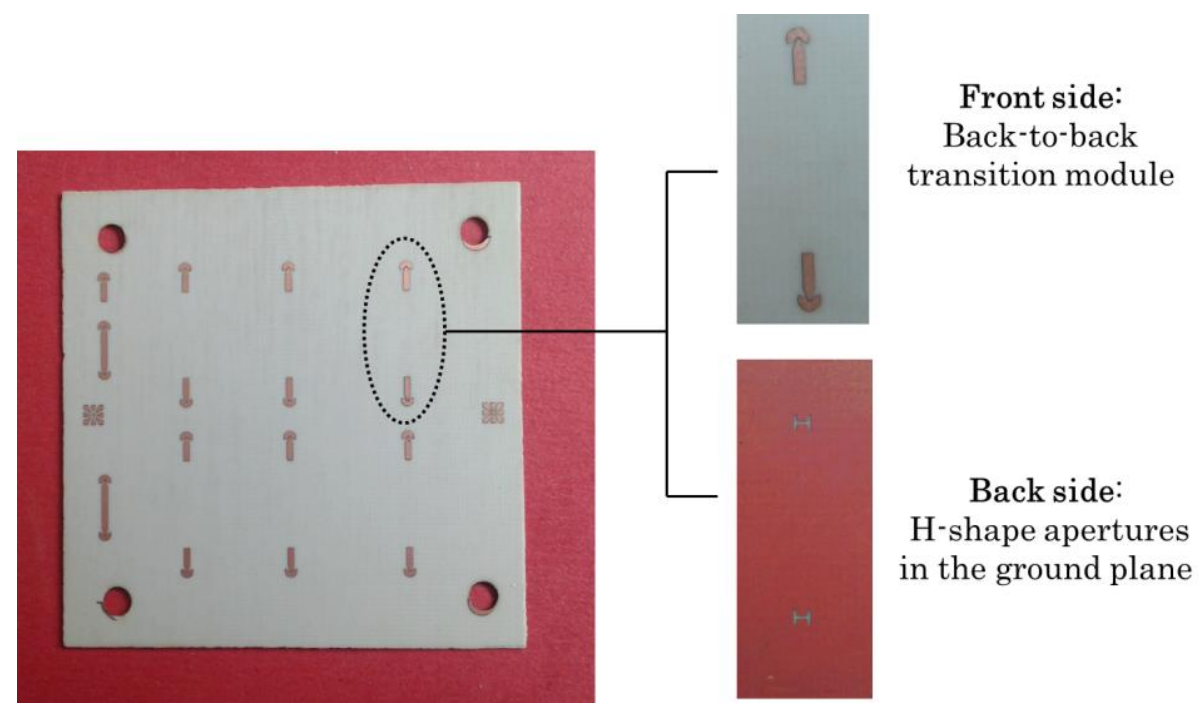

Figure 4.19. Manufactured device with RO4003 including the SIW transitions and a calibration kit at right.

In detail, the CPW radial stub fabricated is represented in Figure 4.20. We can observe the good metallization. The gap between the three probes forming the $\mathrm{CPW}$ is $40 \mu \mathrm{m}$. A picture of $\mathrm{H}$-shape aperture in the ground plane is shown in Figure 4.21. Detailed information about the design and manufacturing process with RO4003 is described in Appendix C.

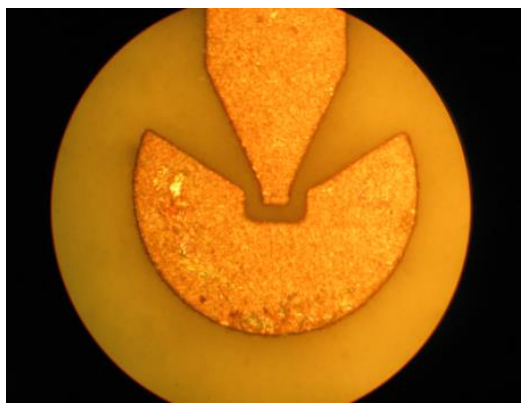

Figure 4.20. CPW radial stubmicrostrip transition.

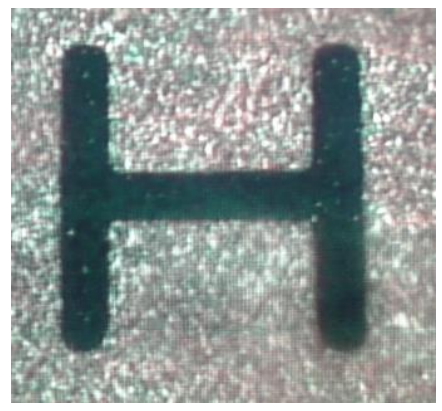

Figure 4.21. H-shape aperture.

\subsubsection{Measurements}

For the device characterization, measurements will be done by two GSG (Groundsignal-ground) probes that contain the probe transition from coaxial to coplanar waveguide. The coaxial is connected with the network analyzer. Probe pitch of the coplanar waveguide probes is $150 \mu \mathrm{m}$. (Figure 4.22). TRL (Through- 
Reflection-Line) calibration technique is employed. The calibration kit has been manufactured jointly with the other gap waveguide structure.
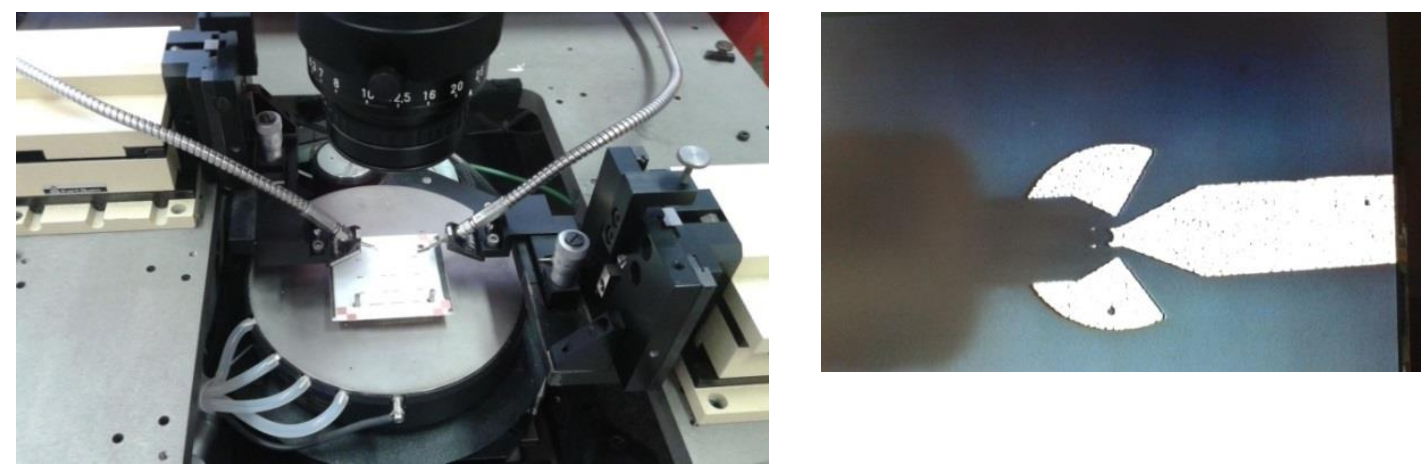

Figure 4.22. RF probe station measurement and GSG (ground signal ground) probe with the coplanar to microstrip transition.

Results of the measurements and a comparison with the computed results are depicted in Figure 4.23. The prototype has been designed with air gap height equal to $200 \mu \mathrm{m}$. However, it appears after retro-simulation that the effective height is equal to $300 \mu \mathrm{m}$. Varying this height induces impedance structure variations, and the transition with the apertures produces a mismatching as observed in Figure 4.24.

This variation of the gap height is produced because the air gap PCB layer is created with a $200 \mu \mathrm{m}$ RO4003 substrate, but the three parts of the prototype (gap waveguide, cavity layer and transition) are stacked with a $20 \mu \mathrm{m}$ thin film. Assuming small air gaps between each layer, the gap height finally converges to $300 \mu \mathrm{m}$.

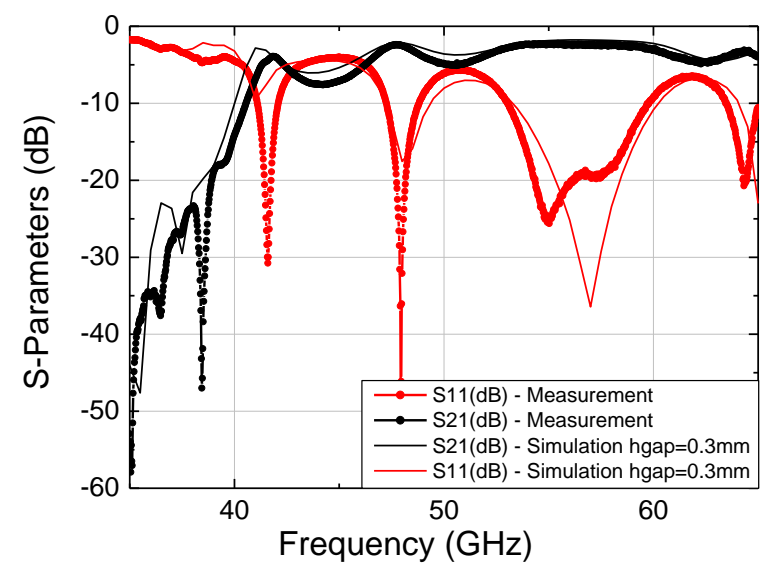

Figure 4.23. Comparison between computed results and measurements for the gap waveguide with RO4003. 

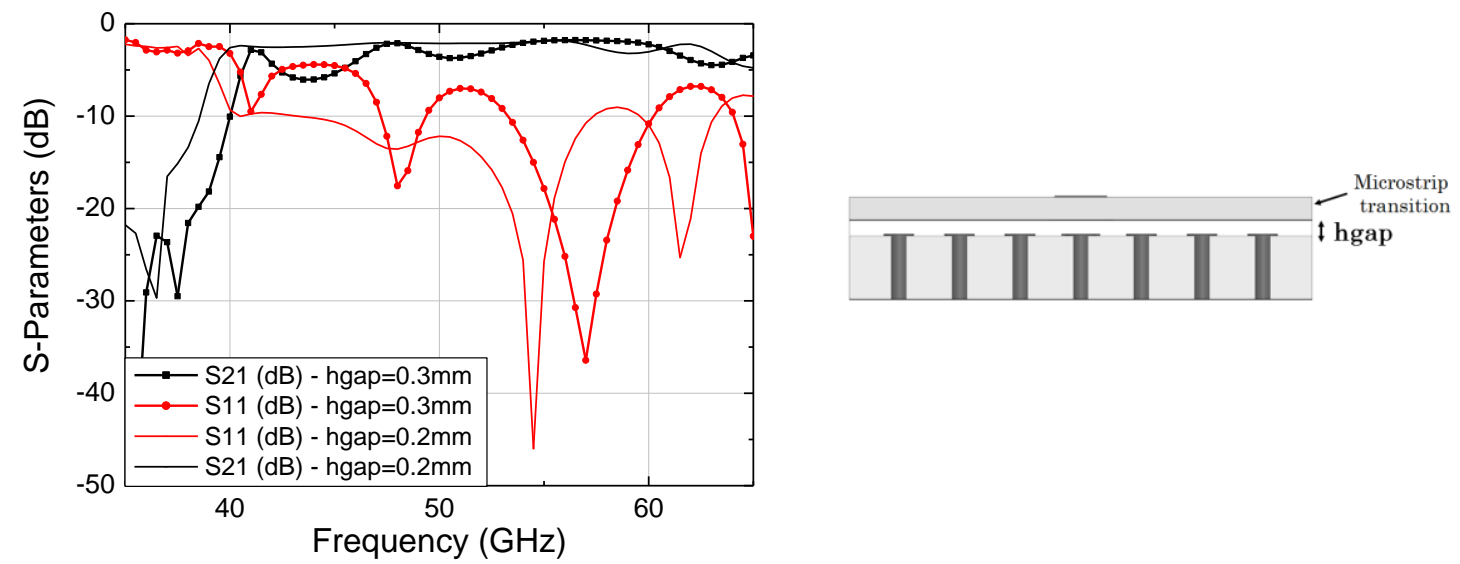

Figure 4.24. Transmission and reflection coefficient for air height (hgap) $0.3 \mathrm{~mm}$ and $0.2 \mathrm{~mm}$ and front view of the gap waveguide, respectively.

Prototype 5 consists of the gap waveguide with two $90^{\circ}$ bends. The interest in analyzing this topology is to show the electromagnetic wave following the ridge in all the discontinuities. The $90^{\circ}$ bends have not been optimized in relation with the neighboring row of pins. The appropriate distance between the transmission line and the lateral vias are deducted by simulations in [74]. In Figure 4.25, the E-field distribution in the upper metal plate of the gap waveguide at $55 \mathrm{GHz}$ is represented.
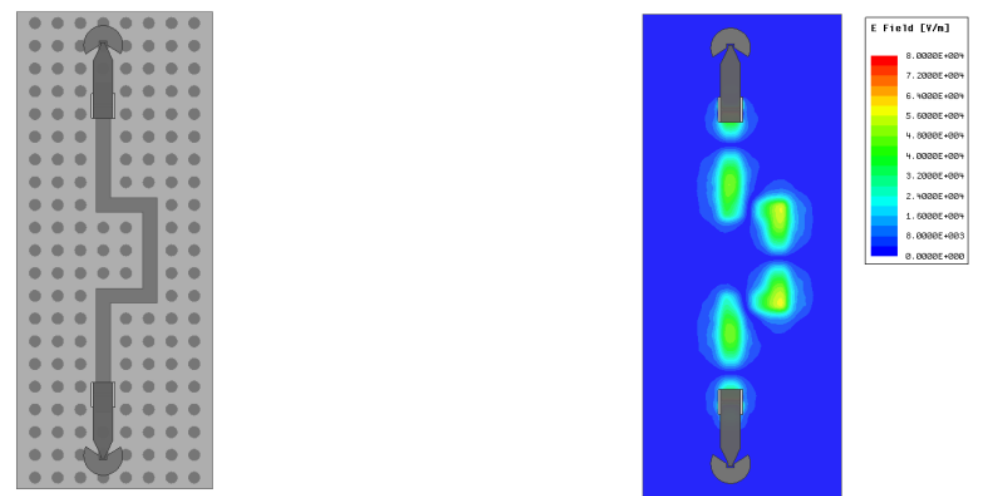

Figure 4.25. Top view of the gap waveguide with two $90^{\circ}$ bends. E-field view in the ground plane with the $\mathrm{H}$-shape apertures at $52 \mathrm{GHz}$.

As in the precedent case, the transition CPW-to-microstrip-to-gap waveguide has been performed for a gap height corresponding to $200 \mu \mathrm{m}$. However, as it has been seen for the previous case, the RO4003 layer that creates the air cavity increase the gap height $50 \mu \mathrm{m}$ or $100 \mu \mathrm{m}$, depending of the stacked parts. 
In order to verify that the height of the air gap of the manufactured prototype corresponds to $250 \mu \mathrm{m}$ (instead of $200 \mu \mathrm{m}$ ); we compare the computed results of transmission and reflection coefficients with the measurements in Figure 4.26. A good agreement between measurements and simulations is shown. Nevertheless, the mismatch produced varying the gap height, makes the response worse.

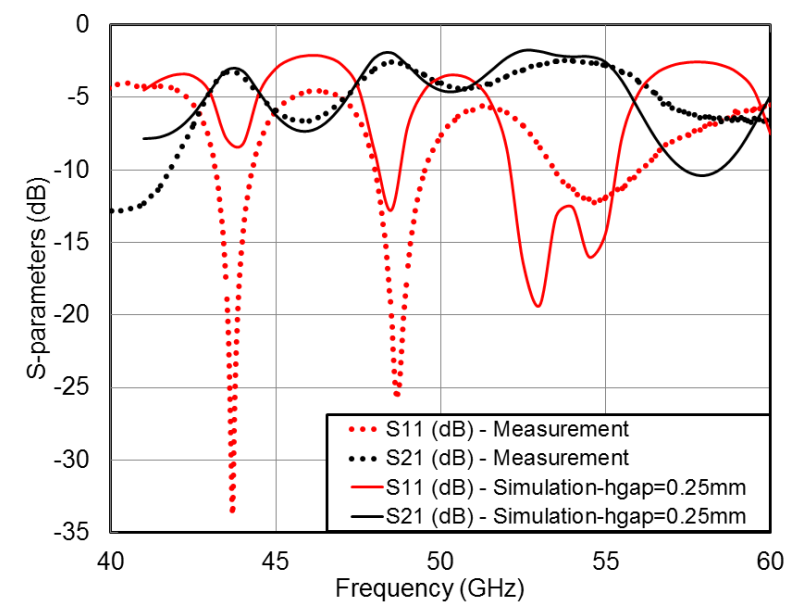

Figure 4.26. Computed results and measurements of the gap waveguide with two 90 bends.

In this section, design and measurements of the gap waveguide prototype using multilayer PCB have been presented. Measurements and computed results are in agreement with only a shift in frequency due to the control process of the air cavity. The important impact of the gap height in our structure is a critical point in PCB multilayer techniques. For better results, industrial process is needed to obtain better performances in terms of dimensions. Dependence with the gap height is explained by means of the characteristic impedance Zc of the structure. Varying the gap height hgap, Zc changes. For example gap waveguide has been modeled with hap $=200 \mu \mathrm{m}$, if we compute the characteristic impedance by the methods described in Section 2.2.2, Zc is equal to $83.17 \Omega$ (with ZPI method) and $82.94 \Omega$ ( ZVI method). It means characteristic impedance around $83 \Omega$. However, in the case of hgap $=300 \mu \mathrm{m}$, the computed characteristic impedance is around $60 \Omega$. Changes in the gap height lead to a change in characteristic impedance which produces a mismatch in the transition.

\subsection{Gap waveguide design using LTCC technology}

Design of the gap waveguide using LTCC technology only has been validated in a recent publication [75], for $\mathrm{W}$-band antenna applications. 
The technological parameters are taken into account. Dimensions are shown in Table 4.2. The geometry and the front view of the design are represented in Figure 4.27. The substrate thickness covering the pin surface is composed by nine LTCC layers being the thickness of each co-fired layer $75 \mu \mathrm{m}$. The dispersion diagram of the structure is depicted and the stop-band region corresponds to [48.8-69.9] GHz (Figure 4.28). As explained in Section 2.2.1, the structure gap height can be reduced to increase this band.

Table 4.2. Dimensions of the gap waveguide design using LTCC substrate.

\begin{tabular}{|ccc|}
\hline \multicolumn{3}{|c|}{ Geometrical dimensions for the gap waveguide with LTCC } \\
\hline Pin diameter & $\mathrm{d}$ & $200 \mu \mathrm{m}$ \\
\hline Patch diameter & $\mathrm{d} 2$ & $400 \mu \mathrm{m}$ \\
\hline Spacing of the pins & $\mathrm{p}$ & $800 \mu \mathrm{m}$ \\
\hline Dielectric & LTCC(ESL41110) & $\varepsilon \mathrm{r}=4.2, \tan \delta=0.004$ \\
\hline $\begin{array}{c}\text { Pin height (or substrate } \\
\text { thickness) }\end{array}$ & $\mathrm{hp}$ & $675 \mu \mathrm{m}$ (9 LTCC layers) \\
\hline
\end{tabular}
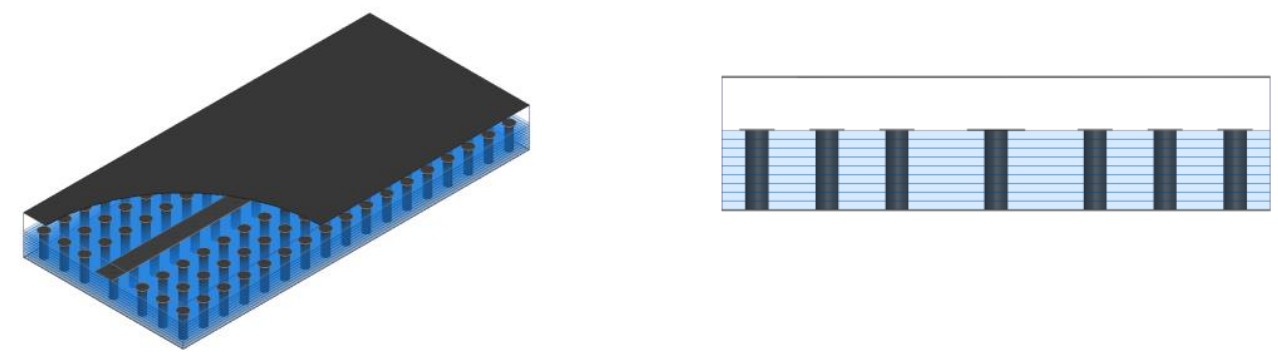

Figure 4.27. Geometry and front view of the gap waveguide with LTCC.

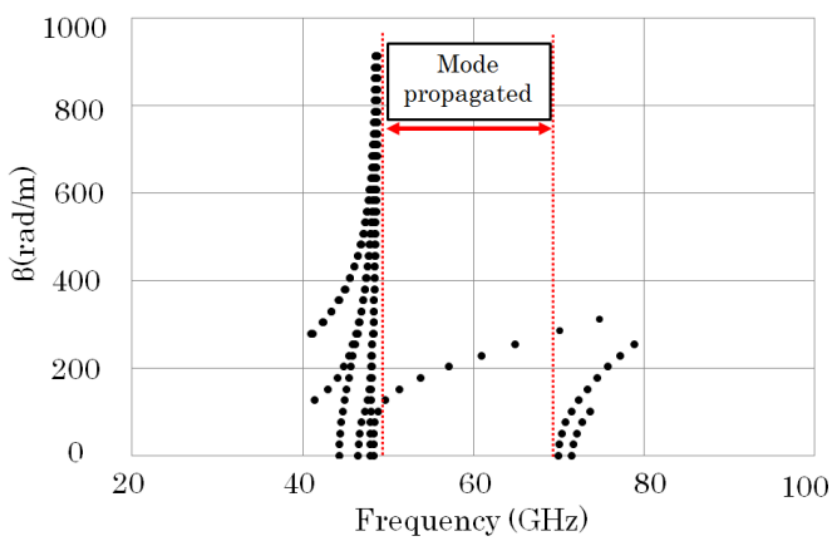

Figure 4.28. Dispersion diagram created by the gap waveguide with LTCC. 
In Figure 4.29, the S-parameters of the LTCC structure are represented. Both cases, with and without losses are considered. The structure is excited by waveguide ports with a TE10 mode. The operating frequency is $60 \mathrm{GHz}$, (in the middle of the stop-band). In Table 4.3, results of transmission losses for two frequencies (55 and $60 \mathrm{GHz}$ ) are shown considering losses (for the conductor (gold) and LTCC material) and without losses. Results demonstrate that introducing conductor and dielectric losses in the materials, transmission loss increases in $0.3 \mathrm{~dB}$.

Table 4.3. Transmission losses with and without losses for two frequencies as reference.

\begin{tabular}{|ccc|}
\hline & Central frequency (GHz) & S21(dB) \\
\hline Without losses & 55 & -0.21 \\
& 60 & -0.43 \\
\hline With losses & 55 & -0.51 \\
& 60 & -0.73 \\
\hline
\end{tabular}

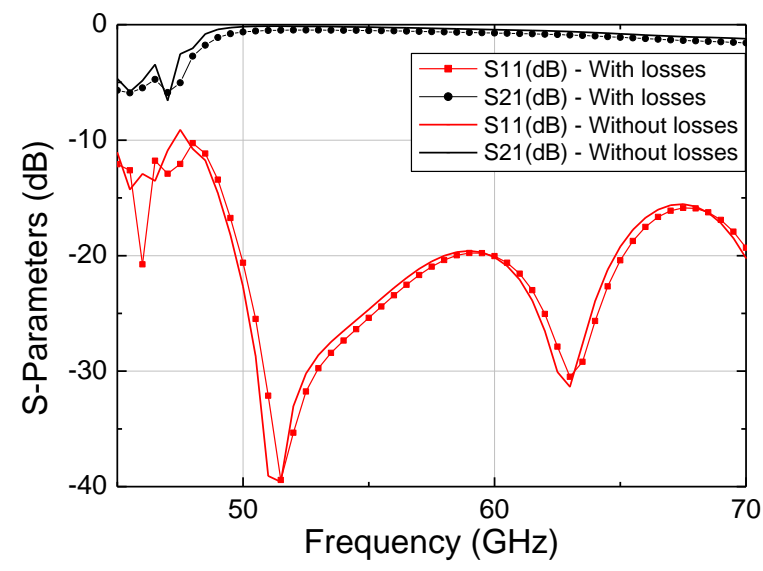

Figure 4.29. Transmission and reflection coefficient for the gap waveguide designed with ESL41110, with and without conductor and dielectric losses.

The CPW-to-microstrip-to-gap waveguide using LTCC is presented. The different sections that contain the complete design of the device are represented: in Table 4.4. The bottom of the first layer supports the ground plane, the first layer is composed by the metallic vias. Then, eight layers containing the metalized pins. On the top of the ninth layer, the patches of the metallic pins and the transmission line are placed. Then, six additional layers creating the internal cavity to generate the air gap of the structure. Above that, on the $15^{\text {th }}$ layer, an upper ground plane incorporating the $\mathrm{H}$-shape apertures is placed. Next, the 
microstrip transition is supported by the next six layers. Finally, the coplanar-tomicrostrip transition is located on the top of the $21^{\text {th }}$ layer.

Table 4.4. Description of the model: Gap waveguide with LTCC.

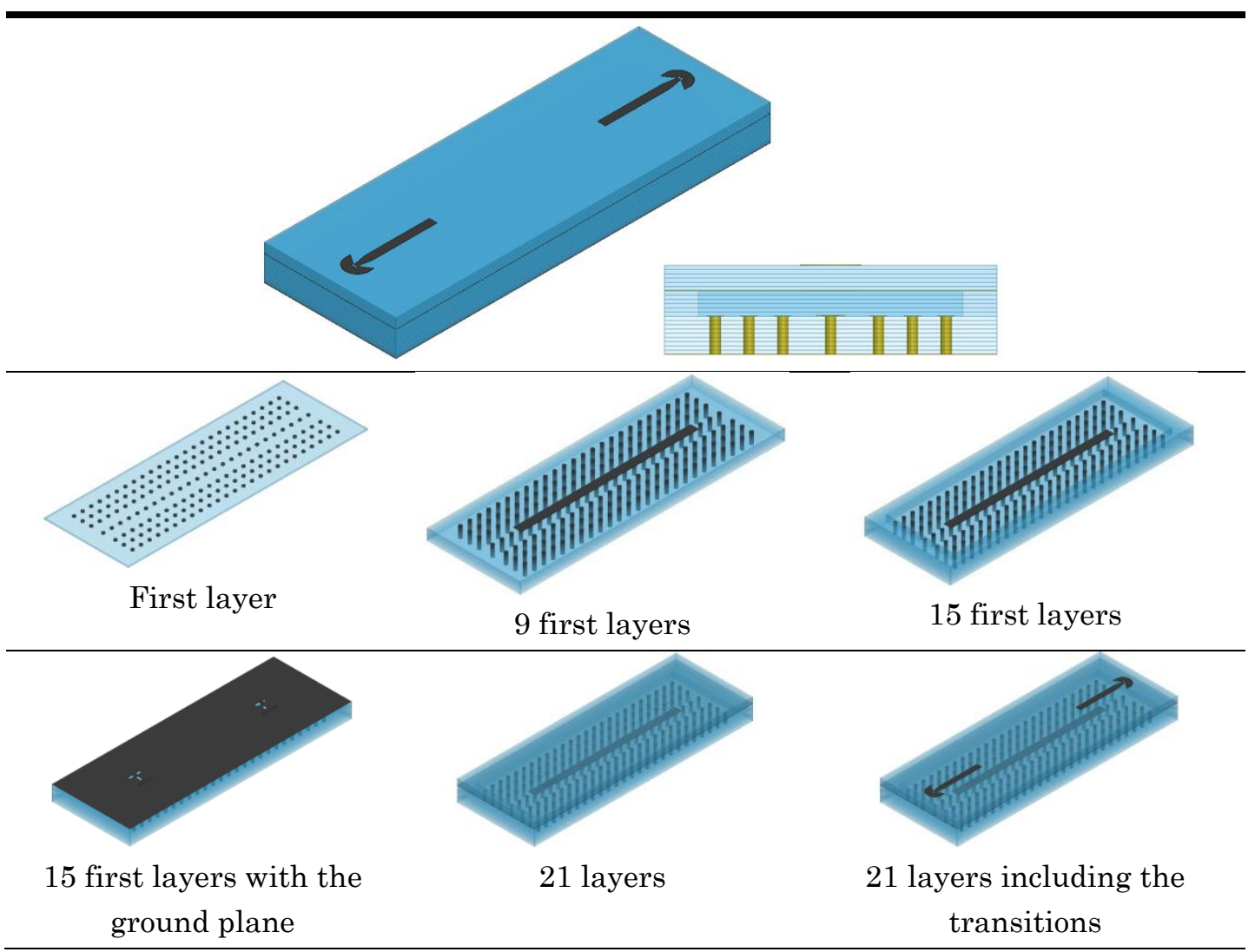

Computed results of the gap waveguide design with LTCC including the transitions are represented in Figure 4.30. Conductor and dielectric losses increase the transmission losses to $0.86 \mathrm{~dB}$. For example, at $52.5 \mathrm{GHz}$, transmission coefficient is equal to $-2.81 \mathrm{~dB}$ and $-1.95 \mathrm{~dB}$ with and without losses, respectively. The insertion losses are due to the gap waveguide losses and mainly, to the transition coupling aperture losses. 


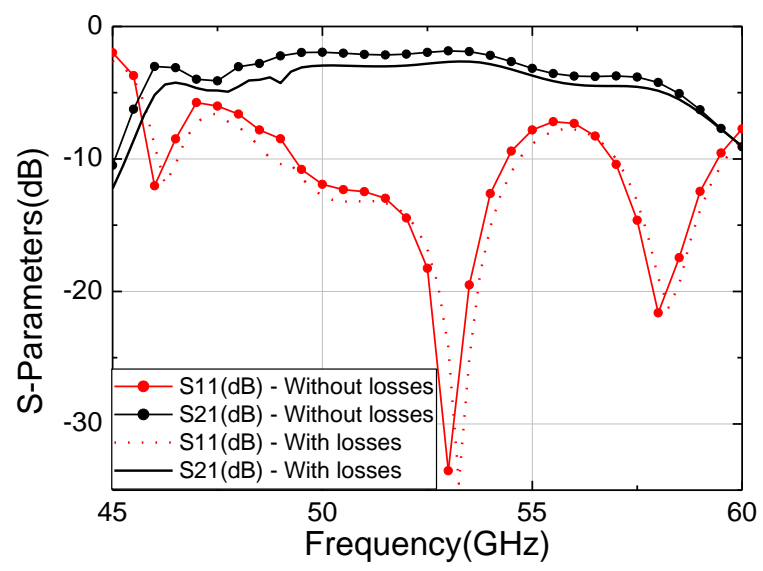

Figure 4.30. Computed results of the complete LTCC structure, including gap waveguide and transition

The 3D plot of the electromagnetic field is represented in Table 4.5. At $55 \mathrm{GHz}$ E-field is completely confined between the transmission line and the upper metal plate (this plate corresponds to the ground plane with the $\mathrm{H}$-shape apertures). Outside this band, the E-field is dispersed over the entire air cavity.

Table 4.5. E-field propagated in the air gap for three specific frequencies: $45 \mathrm{GHz}$ (outside the stop-band), $52 \mathrm{GHz}$ (in the middle of the stop-band) and $62 \mathrm{GHz}$ (outside the stop-band).

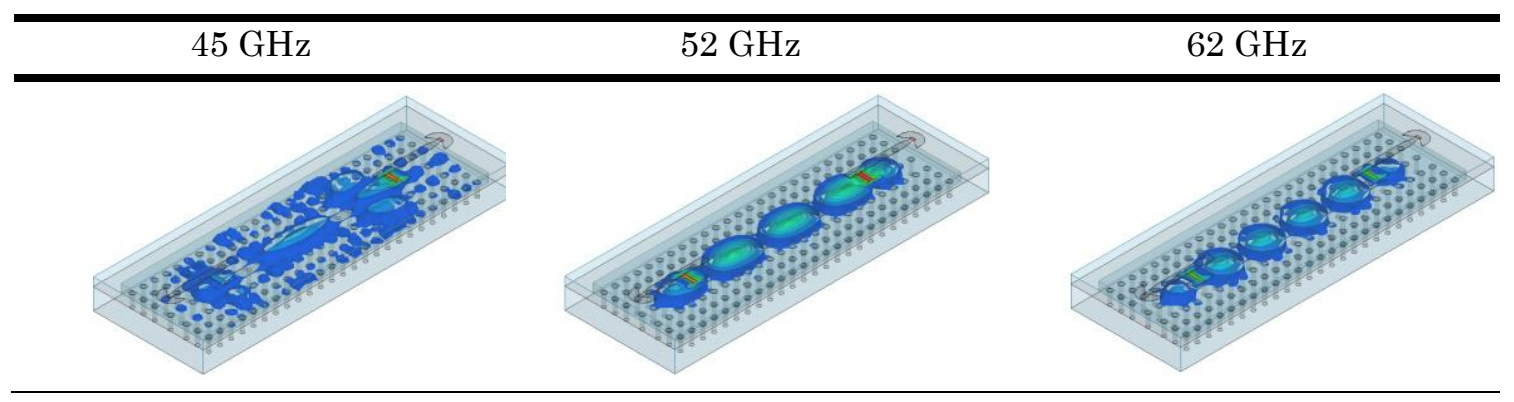

\subsubsection{Manufactured prototype with LTCC}

In this section, first of all the fabrication process of LTCC at the laboratory in Télécom Bretagne will be shown. Next, some technological problems in the manufacturing of the gap waveguide will be presented. Later, design and fabrication of external and internal cavities will be explained. Finally, the gap waveguide manufactured with LTCC is explained according to the dimensions presented in previous section. 
A complex step in the LTCC process is the creation of cavities and channels. In Section 4.5.2, some experiences about internal and external cavities are shown and the technological problems are explained in detail.

\subsubsection{Cavity tests}

One of the main challenge and interest of LTCC technology is the creation of cavities and channels. In our laboratory, for the cavity manufacturing we use Sacrificial Volume Materials (SVM) that is temporary inserted. SVM supports and defines cavities during high pressure lamination process. The elimination of SVM from LTCC module takes place by dissociation during co-firing.

A Sacrificial layer of ESL 49000 is used to prevent the deformation of a capacitive embedded cavity during lamination and sintering process. This material is designed to be burned out in the temperature range of $600^{\circ} \mathrm{C}-800^{\circ} \mathrm{C}$.

In Figure 4.31, the flowchart of the manufactured process of cavities is represented.

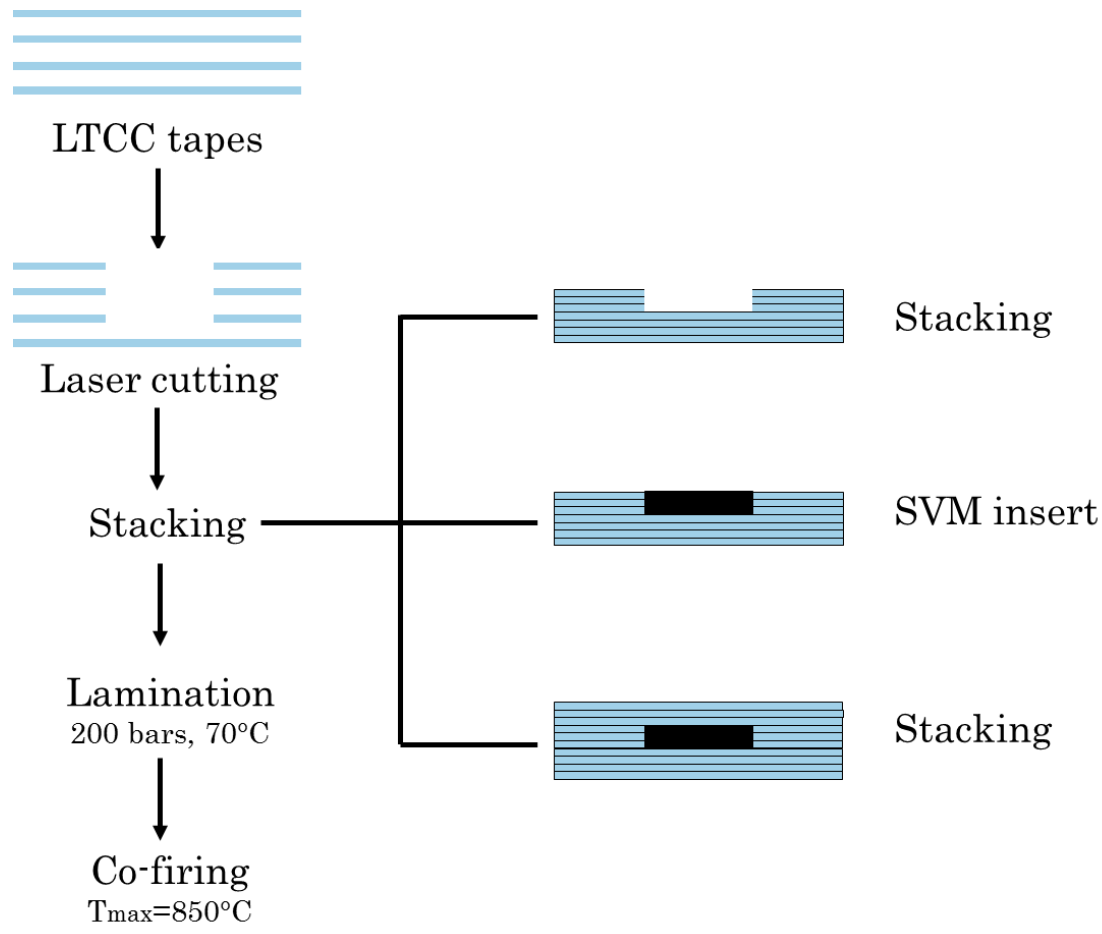

Figure 4.31. Flowchart of cavity fabrication process. 


\section{External cavities}

For the tests of the external cavities, some shapes have been designed as it is shown in Figure 4.32. Four layers under the cavity are needed to avoid deformation. The thickness of the tested cavities is the equivalent of three LTCC layers. Some shapes have been considered, such as a square, a circle and polygons. The manufactured prototype is shown in Figure 4.33.

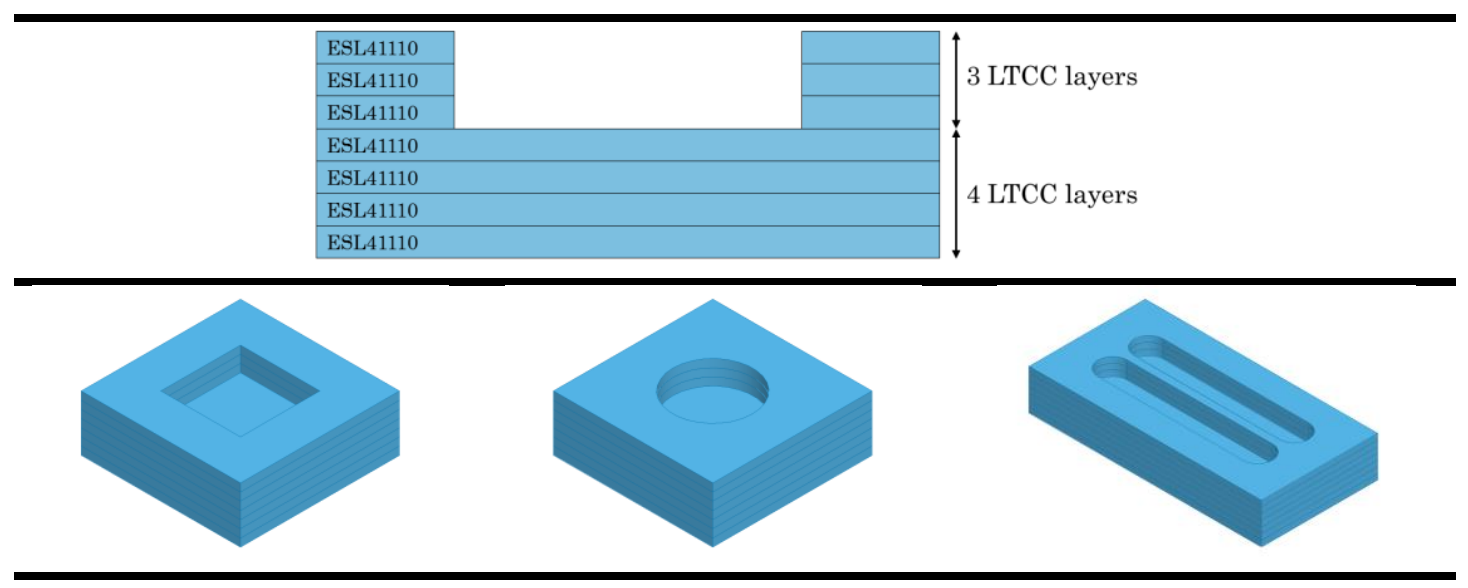

Figure 4.32. Cross-section of the LTCC open cavity.

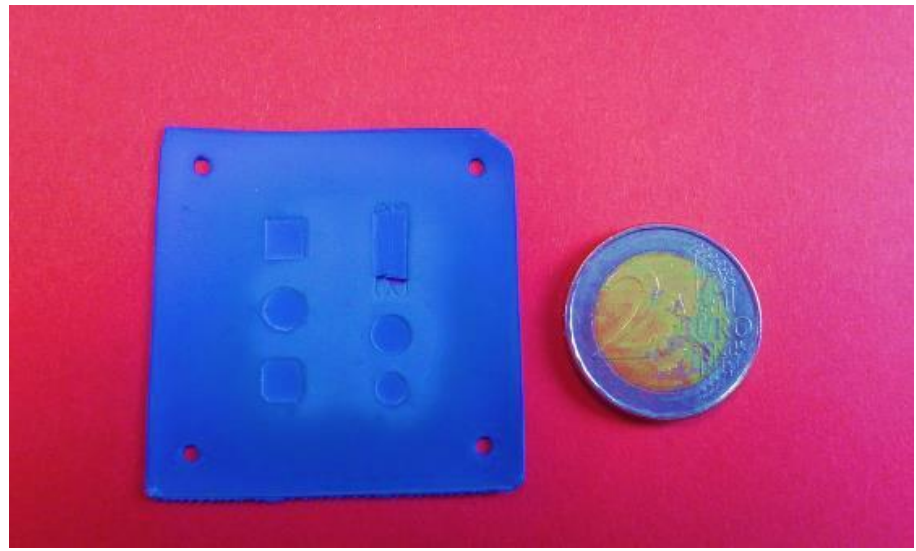

Figure 4.33. Six open cavities included in the LTCC prototype.

Pictures of the open cavities before and after co-firing are represented in Table 4.6. The relationship between the obtained cavity dimensions to expected dimensions is depicted in Figure 4.34. 
Table 4.6. Pictures of the open cavities before and after co-firing.
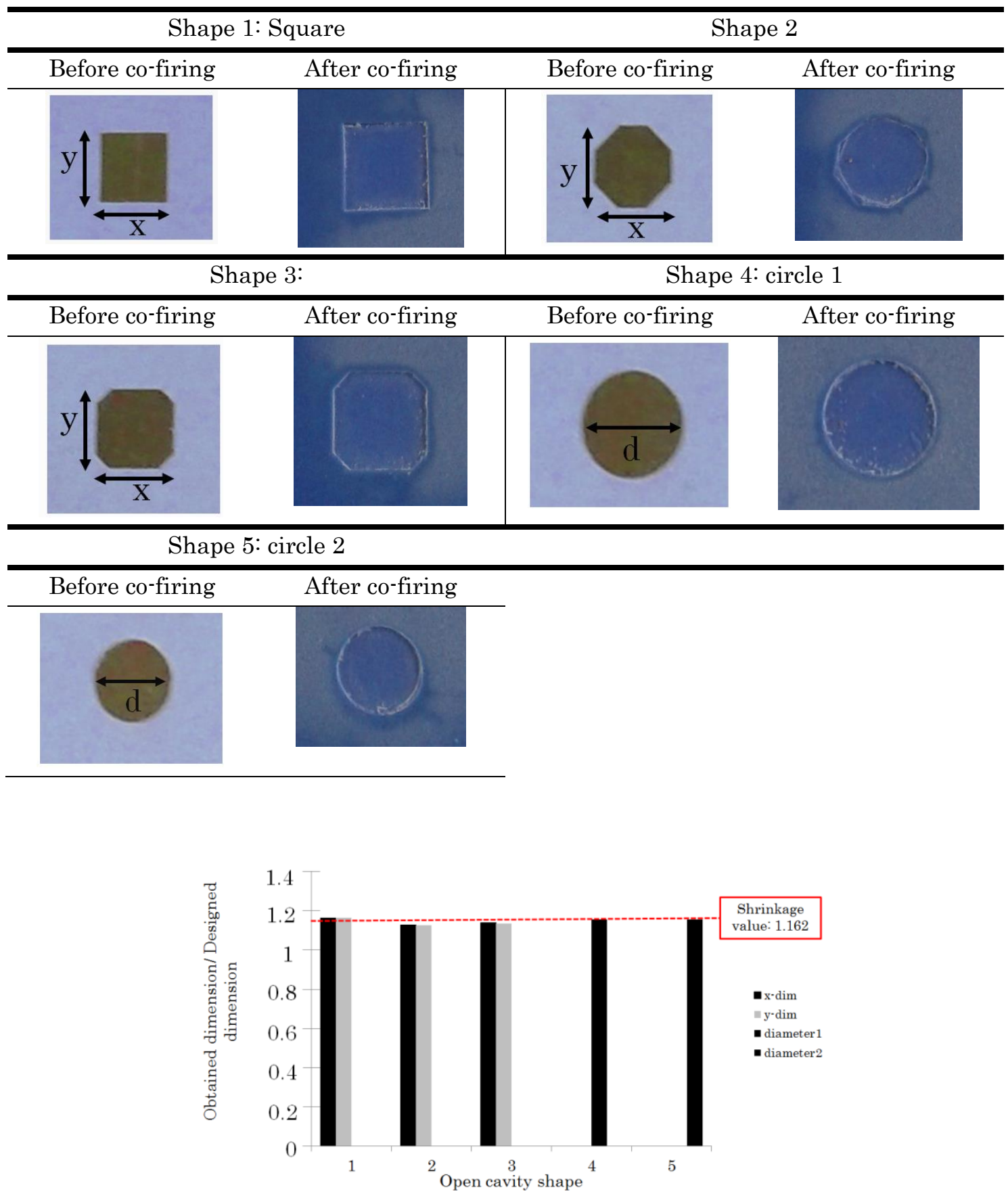

Figure 4.34. Relationship between obtained and designed dimensions for each open cavity.

As can be seen in the Figure 4.34, the difference between obtained and designed dimensions corresponds to the shrinkage value studied for the LTCC 
tape ESL41110 which has a value of 1.162. Apart from this, no significant deformation is shown in the dimensions of the open cavity.

\section{Internal cavities}

With the purpose of controlling the air gap of the gap waveguide structure, and filling it with fluid for its characterization, some tests have been done to analyze shrinkage deformation of such cavities.

The geometry of the cavities is shown in Figure 4.35. A first design consists on a cavity with two holes for exiting fugitive material. The other one is completely closed. Manufactured prototype with both cavities is shown in Figure 4.36.

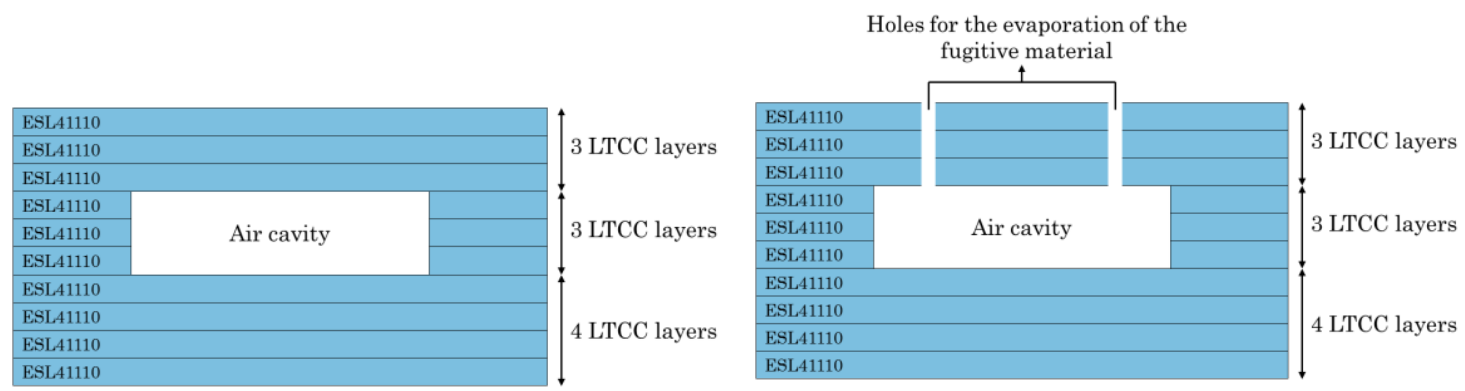

Figure 4.35. Geometry of the internal cavities.

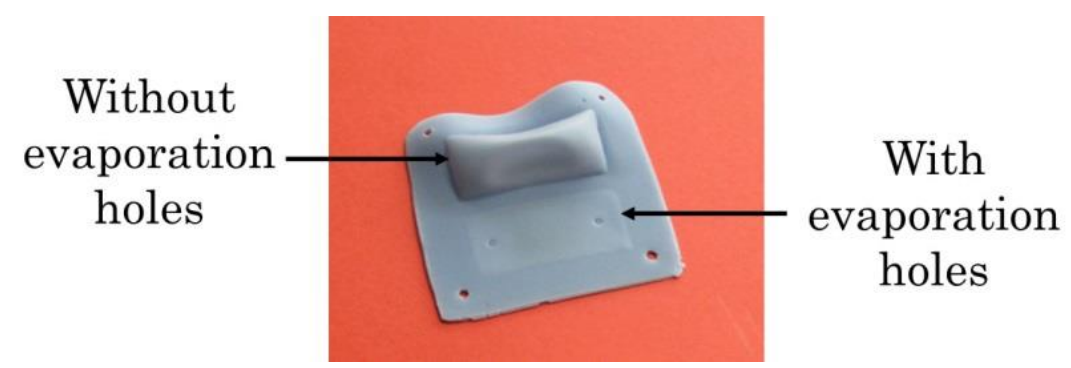

Figure 4.36. Manufactured prototype with two internal cavities: one with holes for the SVM evaporation and other without them.

We can observe an important deformation in the case of the cavity without holes. To explore the deformation and the performances inside the cavity, a laser cutting has been made as it is shown in Figure 4.37. We can see the SVM inside the cavity. 

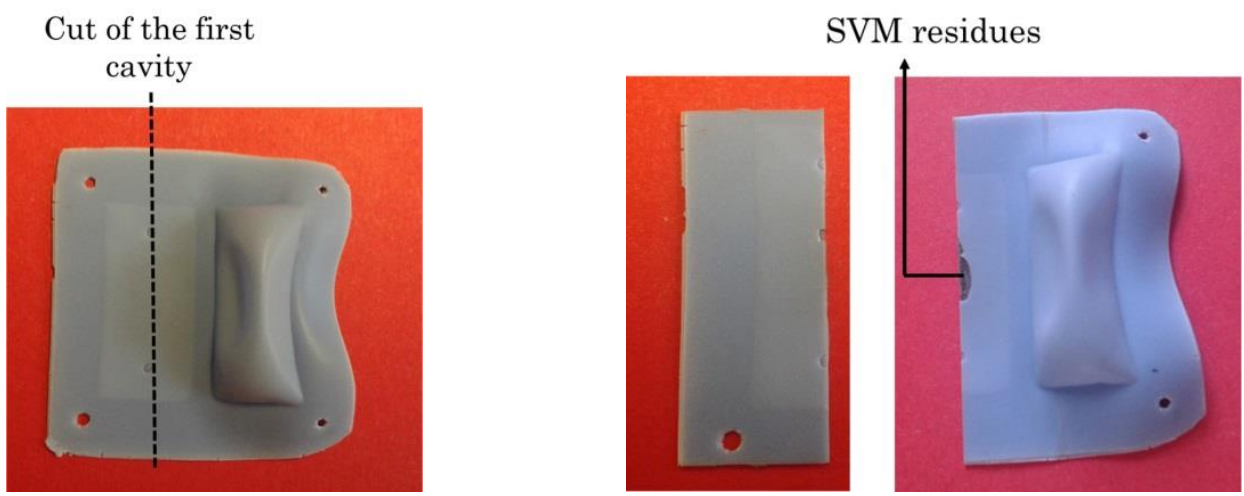

Figure 4.37. Laser cut of the first cavity.

For a further study, we have created a resin base to introduce the cut part of the first cavity and to analyze at the surface of the resin base, the cavity dimensions (Figure 4.38-a). In Figure 4.38-b, the beginning of the cavity is shown. We can observe in this part, that all the SVM has been evaporated. In Figure 4.38-c, the middle of the cavity is analyzed. In this case, we can see the deformation due to lamination, with also the fugitive material (SVM) inside. Because of this sagging, the thickness is reduced with an $80 \%$ ratio.

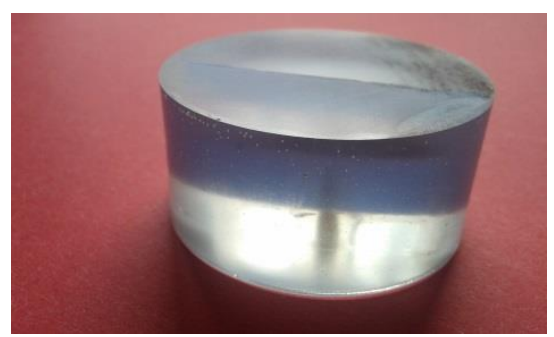

a)

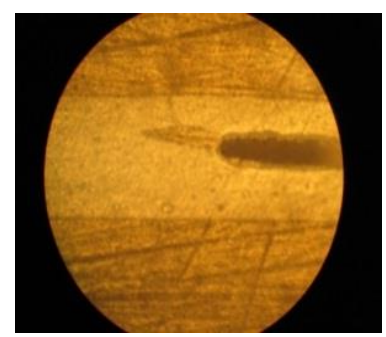

b)

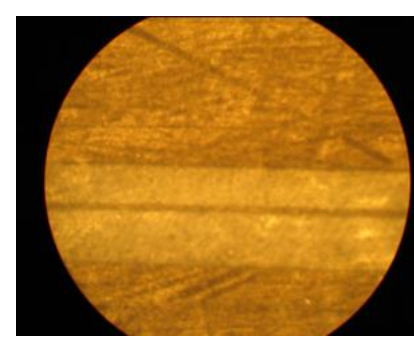

c)

Figure 4.38. Picture of the internal cavities: a) Resin base to analyze performances inside the cavity; b) First part of the cavity; c) At the center of the cavity.

From this test, some technological problems occurred, namely, a percentage of the SVM is concentrated inside the cavity and in the case of the cavity without holes, all the SVM is inside the cavity. To overcome these issues, we propose several conditions:

- It is necessary to add more holes for the SVM evaporation.

- A change in the temperature profile can allow adding more time for the SVM evaporation.

- A reduction of cavity dimensions ( $\mathrm{x}$ and $\mathrm{y}$ ) would permit a better control of the cavity process. 
With the characteristics mentioned above, another test has been done to study the cavity deformation. This test is composed by three LTCC internal cavities. The structure of one of these cavities is shown in Figure 4.39. The modifications included in this test are the following one: we have increase the number of holes for the SVM evaporation, the thickness of the cavity has been increased the holes diameters do not exceed $400 \mu \mathrm{m}$.
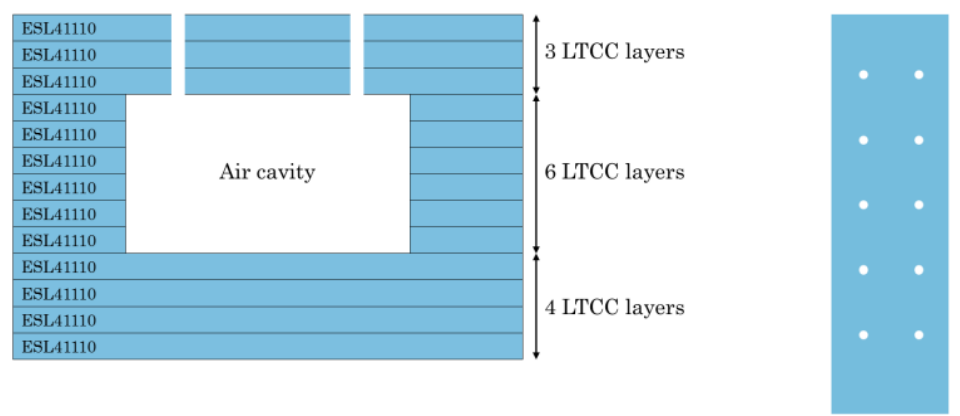

Figure 4.39. Pictures of the second test of LTCC cavities: side and top view.

The manufactured prototype is presented in Figure 4.40-a with the detail for the number of holes and diameter dimensions. In Figure $4.40-\mathrm{b}$, a backlit image of the prototype is shown. In the case of the first cavity, SVM remains inside the $75 \%$ of the cavity volume. However, second and third cavities present good results. All the fugitive material has been evaporated through the holes with diameter of $300 \mu \mathrm{m}$ and $400 \mu \mathrm{m}$.

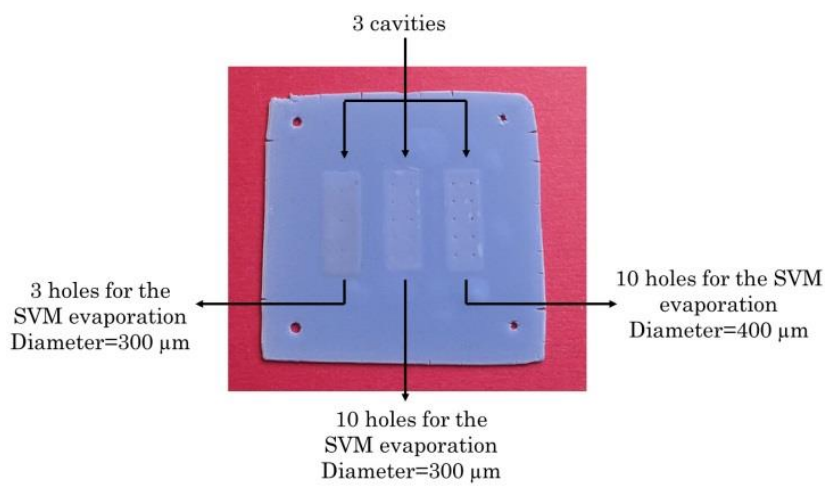

a)

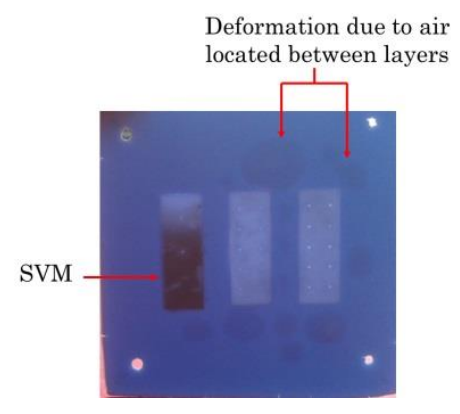

b)

Figure 4.40. Second test for the LTCC manufacturing of internal cavities. a) Manufactured prototype with the three cavities; b) Backlit image of the prototype. 
A cut of the second cavity has been done, the result is shown in Figure 4.41. Dimensions correspond with the theoretical values meaning that fugitive material prevents the shrinkage produced inside the cavity.
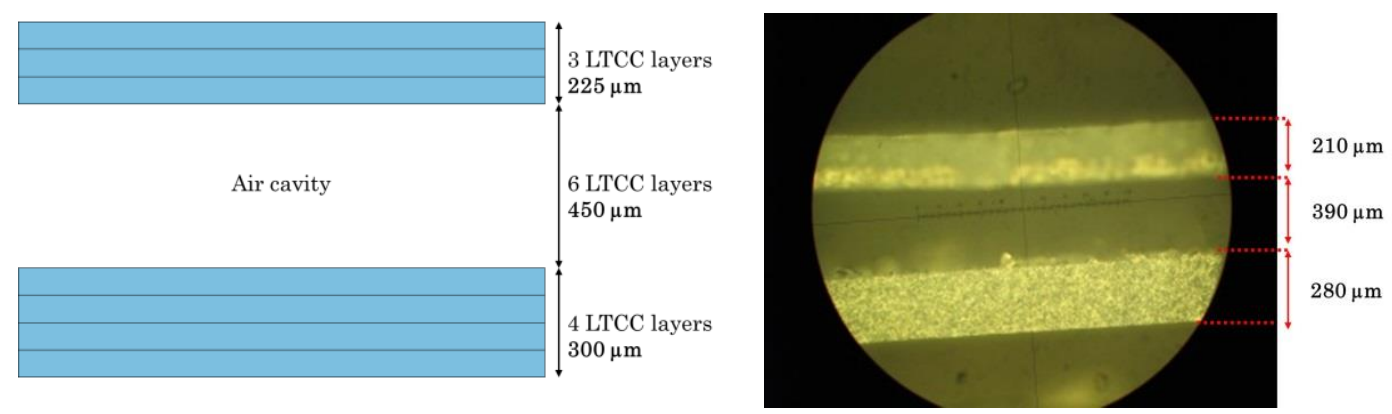

Figure 4.41. Designed and obtained dimensions of the internal cavities thickness.

Although, results of the internal cavities are good in terms of dimensions, we can improve the accuracy of the dimensions of the manufactured internal cavities by: 1). A change of the temperature profile, with an increase of the temperature range where the SVM can be evaporated; 2) An adjustment of the pressure in the lamination process to avoid the deformation of the air located between layers.

\subsubsection{Final LTCC gap waveguide prototype}

Finally, in this section, the prototype with LTCC is manufactured, considering all the technological constraints and design methods described in previous sections.

The design of the gap waveguide with LTCC has been described in Section 4.1.2 and 4.1.4 for the gap waveguide and the transition respectively. The prototype is composed by 21 LTCC layers: nine layers for the metallic pins and transmission line, six layers for the air cavity and six layers for the transition coplanar to microstrip. Cross-section of the prototype is depicted in Figure 4.42. 


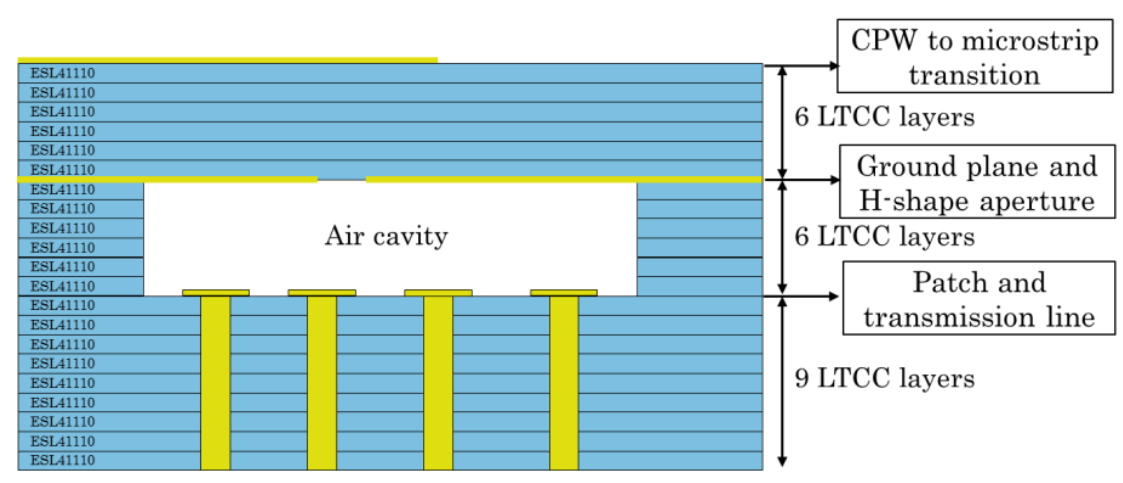

Figure 4.42. Cross-section of the LTCC gap waveguide prototype.

Firstly, the different steps for the manufacturing of the gap waveguide prototype with the LTCC process are explained.

Once slitting, blanking and preconditioning of the LTCC tapes have been done, Next step consists in the holes and cavities creation. The first nine layers of our prototype are composed of 800 metallic pins or vias for each layer. Each via has a diameter equal to $200 \mu \mathrm{m}$. Last six layers, forming the substrate thickness of the microstrip transition, contain several vias for evaporation of the fugitive material inside the cavities (Figure 4.43-left). All the layers contain four registration holes and two alignment holes for stacking. In addition, laser cavities have been cut as it is shown in Figure 4.43-right. The prototype is composed by three gap waveguides, each structure with its air gap cavity. For the gap waveguide resonators, also another cavity is included with the aim to introduce liquid samples for its characterization.
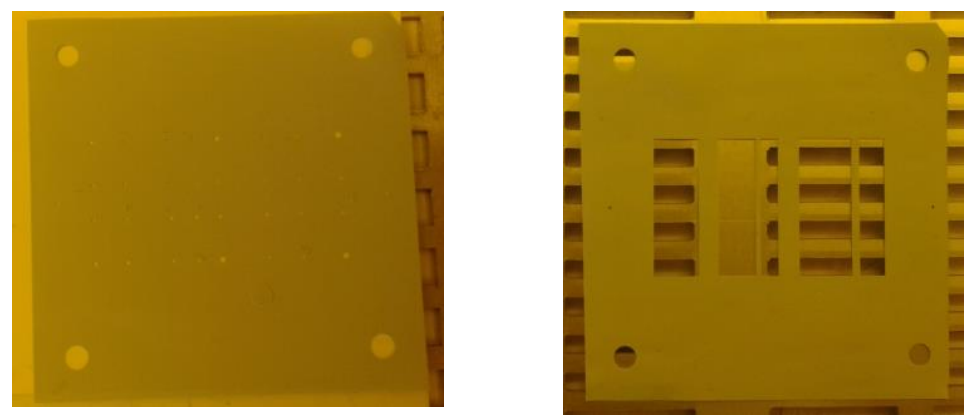

Figure 4.43. Laser cutting: holes and cavities.

Next step consists of the via filling and the serigraphy of transmission lines. This process is done for the filling of holes created with the laser, on the top of the ninth layer. Serigraphy is necessary for patches located on the vias, the 
transmission lines and for the metallization of the two "ground-planes", one in layer one and the other at the bottom of layer 15. Finally, the metallization of the printed transition from coplanar to microstrip is presented (Figure 4.44).
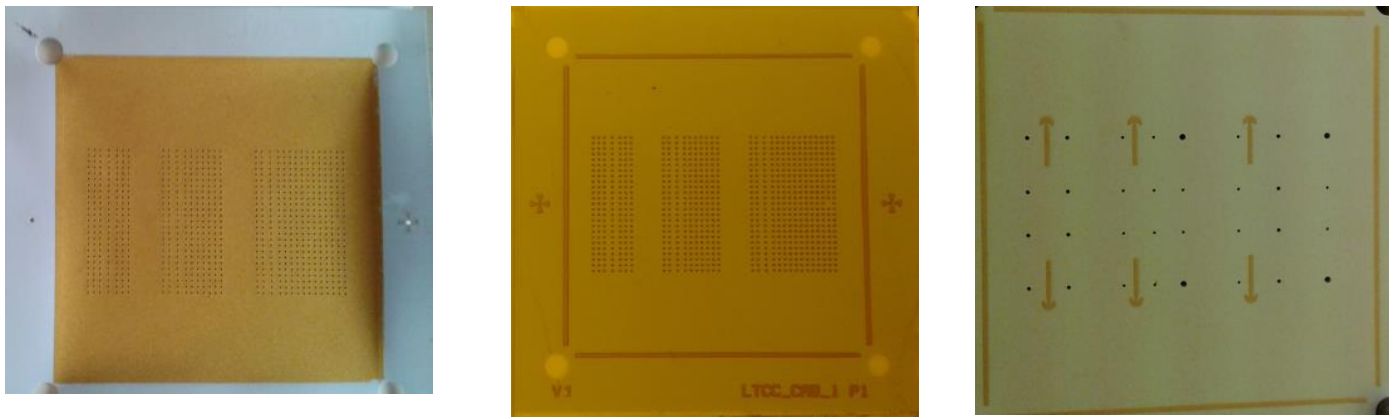

Figure 4.44. Via filling and serigraphy.

The following step in the LTCC process is the stacking of the 21 layers, and the introduction of the fugitive material to avoid deformation in the lamination process (Figure 4.45).
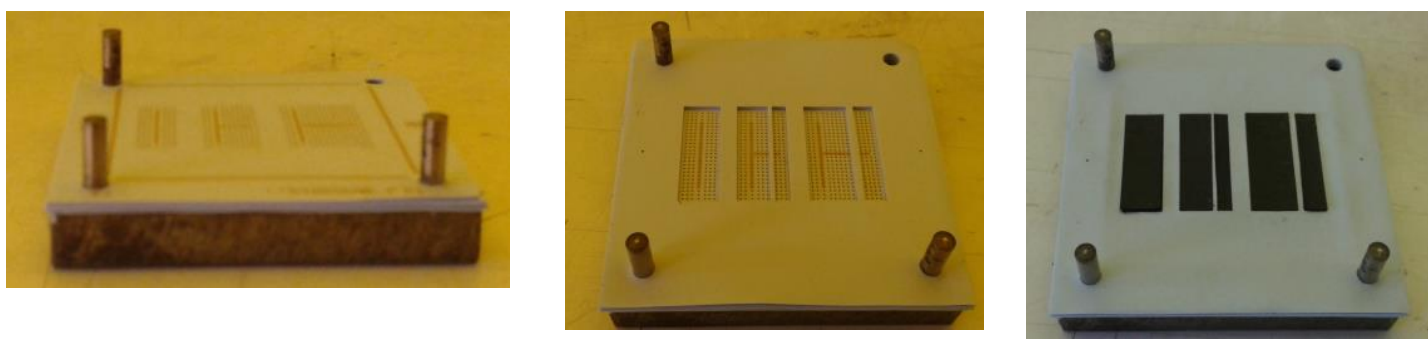

Figure 4.45. Stacking of all the layers.

After that, the prototype is under vacuum for ten minutes and is laminated with the uniaxial press of the laboratory. Finally, it is co-fired according to the LTCC firing profile described in Appendix B.

In a last step, the gap in the coplanar transition is created through laser ablation. In Figure 4.46, the before and after of the ablation process are represented. 

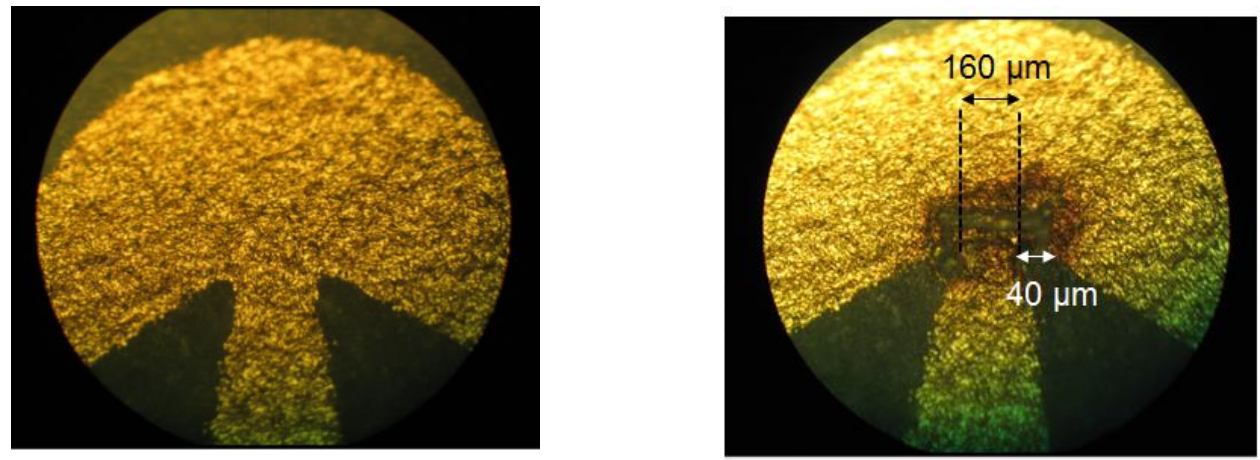

Figure 4.46. Ablation process to create the gap for the CPW transition: before (left) and after (right) ablation.

The final prototype is shown in Figure 4.47. It is worth remarking that some deformations in the registration holes and at the edges are present. This is probably due to the high pressure during the lamination process. Therefore, we can see that the cavity zones are deformed. At the end of this chapter, this deformation will be analyzed in detail.

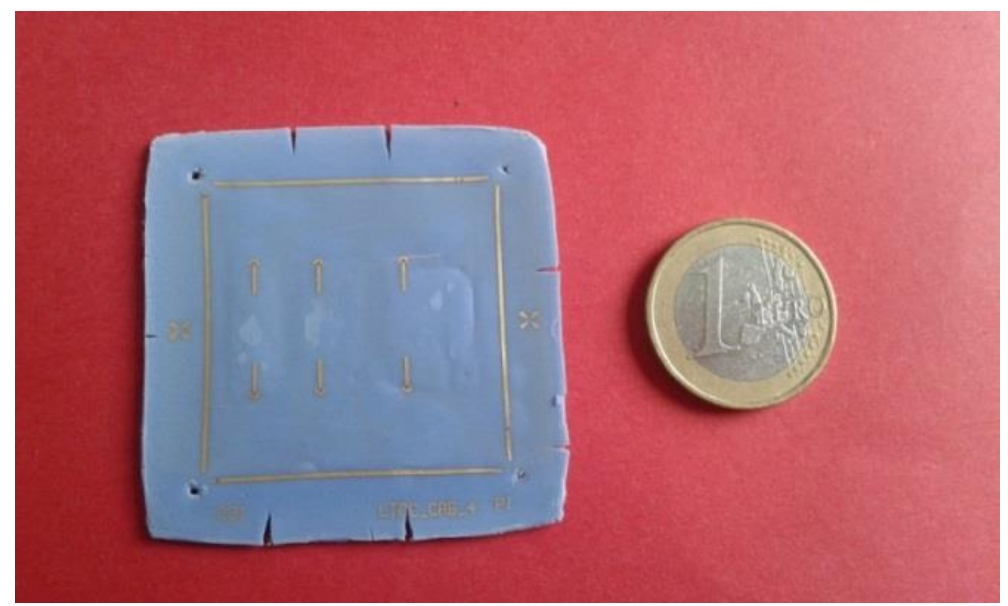

Figure 4.47. Final manufactured prototype with LTCC.

\subsubsection{Measurements of the LTCC gap waveguide prototype}

Measurements have been done with the GSG probe as shown in Figure 4.48. We analyze the first prototype consisting of the gap waveguide described at the beginning of the chapter. 


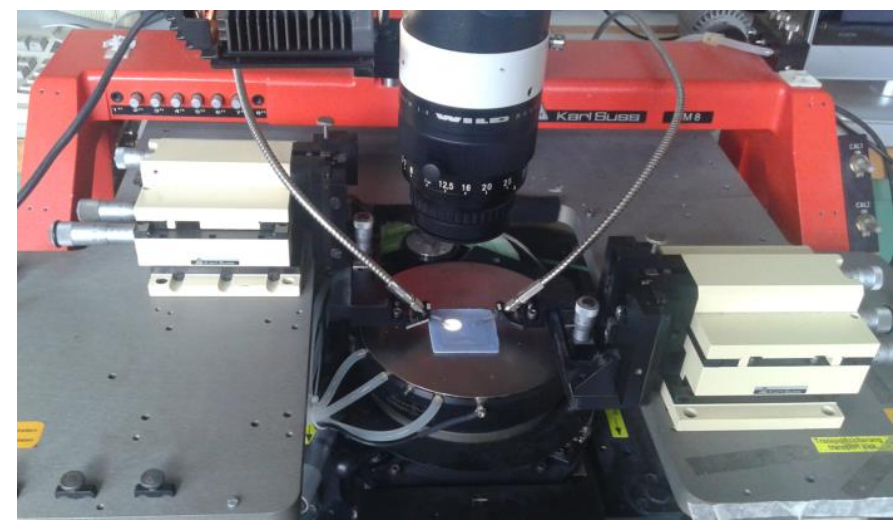

Figure 4.48. Measurements with the GSG probe of the LTCC prototype.

Results of the measurements are depicted in Figure 4.49 and they are compared with the computed results obtained by simulation. As it can be seen, measurements are not in agreement with the computed results. The matching conditions are not acceptable as well as insertion loss, close to $15 \mathrm{~dB}$.

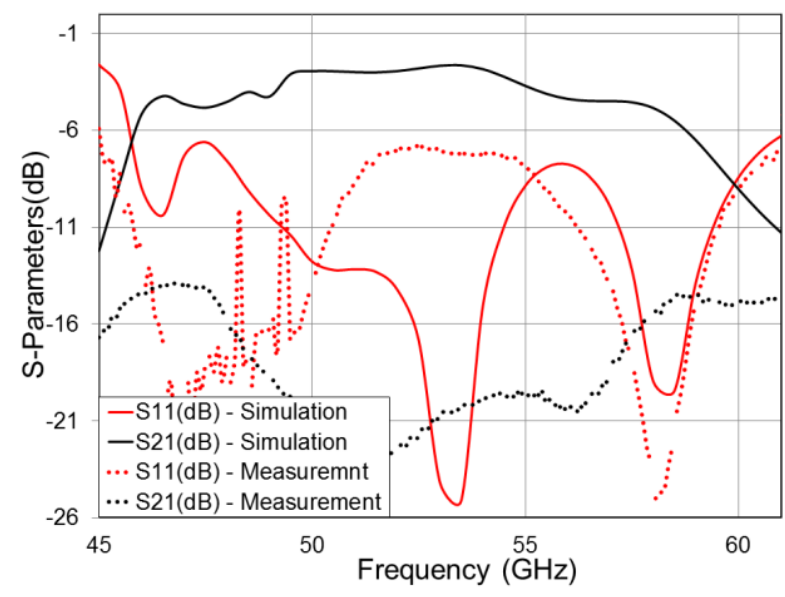

Figure 4.49. Comparison between measurements and computed results of the LTCC gap waveguide.

A cut of the prototype has been done to study the via filling and the quality of the cavities for several cross sections. Moreover, a resin base has been performed as it was done for the study of the internal cavities (Figure 4.50). This resin base allows a better measurement of the dimensions of the transversal cut (height of the pins, thickness of the cavity, via filling, etc.). 

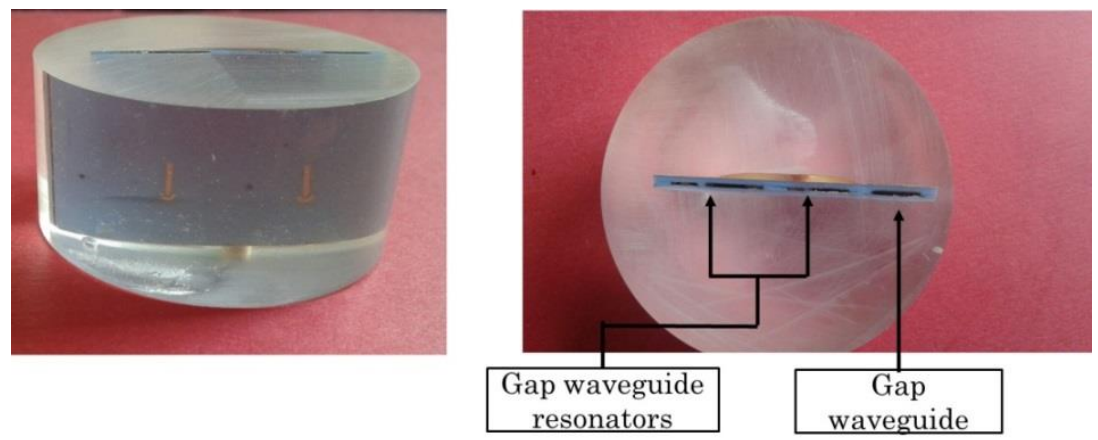

Figure 4.50. Resin base created for measuring the dimensions in the cut plane of the gap waveguide prototype.

The cut plane allows the analysis of the three gap waveguide created in the same LTCC prototype: gap waveguide and two gap waveguide resonators. In this chapter, we are focused in the study of the first gap waveguide.

The cut shows problems that have occurred in the manufacturing process. First, the screen printing of the ground plane has been done. The thickness of this gold layer should be $3 \mu \mathrm{m}$, theoretically. However, a $30 \mu \mathrm{m}$ thickness is measured. Moreover, as it is seen in Figure 4.52, in some places gold has been detached and it creates a short circuit avoiding the propagation between the back to back configurations. This explains, the lower value of the transmission parameter of the Figure 4.49. Another difficulty is related to the via filling. As it is observed in Figure 4.51, the metallization of the vias is not so good, even for the metallic contact between layers.
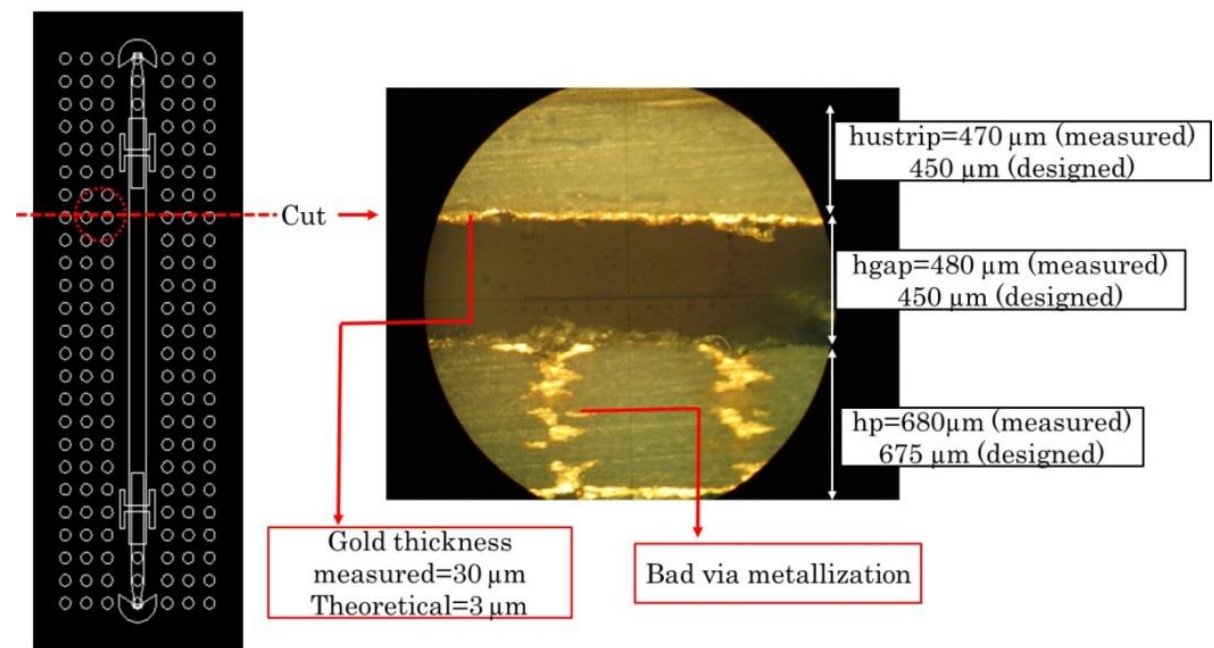

Figure 4.51. Cut of the gap waveguide prototype with LTCC. Dimensions are specified and the problems obtained related to the serigraphy of the gold and the metallization of vias. 


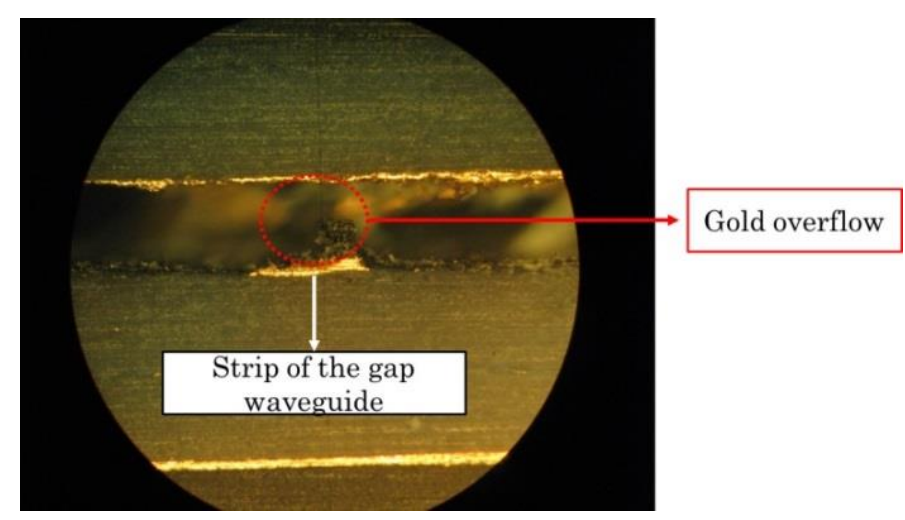

Figure 4.52. Overflow of the gold of the ground plane into the cavity.

Firstly, the via filling is not so good. Furthermore in certain zone there is not metallic contact between the layers to create the via (Figure 4.53). Second problem was the metallization of the ground plane of the microstrip transition. In fact, as it has been explained in Chapter 4, normally the thickness of the gold after the co-fired process is $3 \mu \mathrm{m}$, however in our prototype there are regions where this thickness is $40 \mu \mathrm{m}$, because the gold has overflowed up to touching the via surface, creating a short circuit (Figure 4.54). Therefore no transmission occurs in our structure. Thirdly, other problem is the misalignment between the $\mathrm{H}$-shape aperture and the central axe of the gap waveguide as it is shown in Figure 4.55.

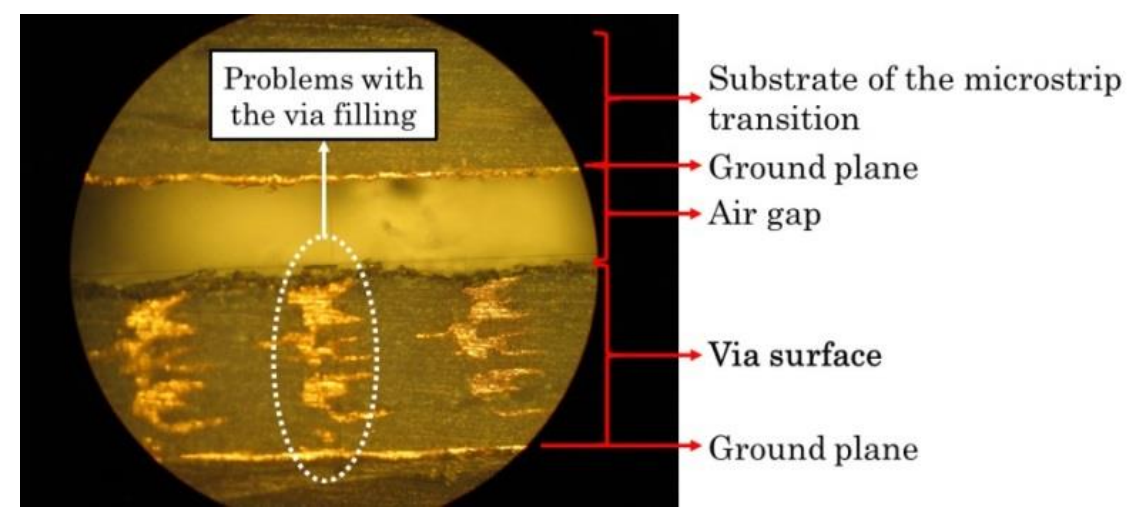

Figure 4.53. Via filling problems. 


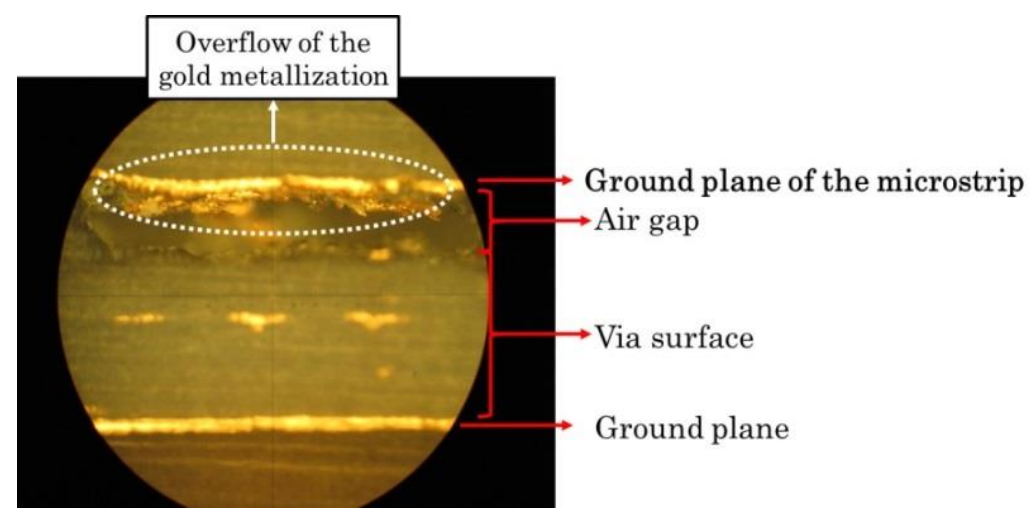

Figure 4.54. Overflow of the gold metallization. Gold thickness $\approx 50 \mu \mathrm{m}$.

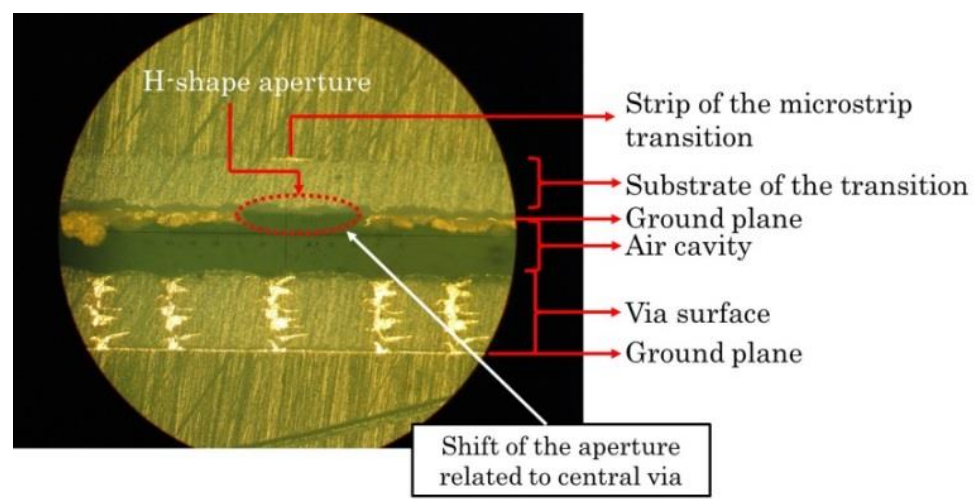

Figure 4.55. Shift of the aperture related to the central via.

\subsubsection{Possible solutions for manufacturing problems}

For the via metallization:

- Exhaustive control of the gold paste viscosity.

- Insertion of metallic "patches" for each layer containing vias, as shown in Figure 4.56 .

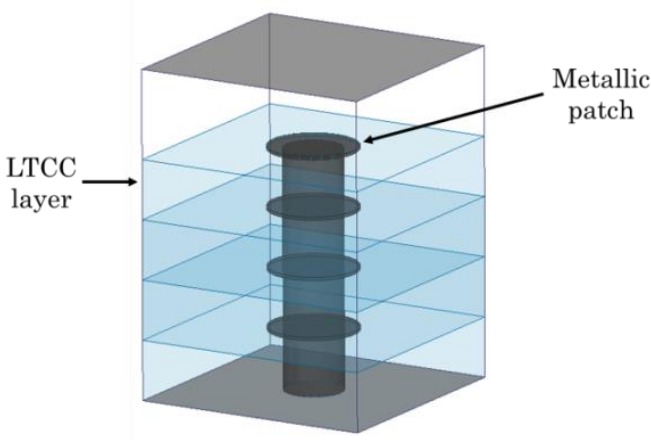

Figure 4.56. Insertion of a "metallic patch" in each LTCC layer to solve the via filling problems. 


\subsection{Conclusion}

This chapter presented the design and manufacturing of gap waveguide prototypes with PCB and LTCC technologies. First, the design of the gap waveguide with RO4003 substrate has been explained. Then, the feeding transition has been analysed. This transition consists of a microstrip to H-shape aperture-coupled to the gap waveguide. H-shape aperture reduce leaky waves and reduces the dimensions in comparison with rectangular aperture Therefore, a coplanar probe is introduced in a back to back configuration for CPW probe measurements. As a result, measurements are good in comparison with simulations for the gap waveguide using RO4003 substrate. Nevertheless, the dependency between the gap height and the characteristic impedance has been observed, being a limitation for the PCB multilayer techniques. For this reason, other manufacturing process as LTCC becomes a candidate to overcome these difficulties in terms of control of the cavities.

For this reason, a work in the laboratory to control the internal and external cavities manufacturing has been done. Manufactured dimensions of the external and internal cavities have been shown to be in agreement with the design. Several manufacturing considerations have been explained for the cavity formation process.

Finally, the gap waveguide prototype manufactured using LTCC, more exactly ESL41110 tape, has been shown. Measurements have manifested that problems during the manufacturing process have occurred. These problems are related to the serigraphy of the gold and the via filling. We can summarize these problems into: problems with the via filling, excess of pressure in the lamination process and shift in the alignment. To overcome these difficulties, in terms of via filling it is necessary to improve the process using the screen printing because with the optical camera of our system, it is complicated to view and align vias in the screen and in the substrate. Another solution consists of introducing metallic patch on the top of each LTCC layer containing vias. It is necessary also to adjust the quantity of paste viscosity and pass number. In the case of the lamination process, it is interesting to study other solutions as isostatic process using heated water. This method is more complex than uniaxial technique but presents less deformation in the substrate density. 


\section{Chapter 5}

\section{Gap Waveguide resonator for microfluidic applications}

\subsection{Introduction}

In Chapter 1, the motivation and the interest of microfluidic applications was explained. The integration of microwave sensing into microfluidic systems is based on the analysis of the electromagnetic properties of a fluid to obtain information about its composition. Currently, the frequency range of microfluidic sensors is limited to $40 \mathrm{GHz}$ [76]. However, experimental results presented by Tong Chen [77] show an interest in using millimeter-wave to assess concentration of living lymphoma cells by conductance measurement.

Current solutions are based on a local perturbation of transmission line or resonator with coplanar waveguide. However, the technical constraints in millimeter-wave limit the frequency band. To provide a new technical solution, we propose in this work the use of the gap waveguide technology [78]. This technology has been validated to work at the millimeter-wave band in last publications [79]. It presents interesting advantages for the fluidic characterization because we can create fluidic channels in the maximum e-field region. 
In this chapter, we propose a type of sensor based on the gap waveguide technology taking advantage of the interaction between the fluid and the electromagnetic response in the region where the maximum E-field is concentrated. Moreover, this technology can minimize the RF access losses. The chapter is organized as follows: first, the design of the gap waveguide resonator is presented. This section includes the design and the simulation including the LTCC cavity created for the fluid. Next section shows a comparison between gap waveguide and Substrate Integrated Cavity in terms of sensitivity. Finally, manufacturing and measurements of gap waveguide resonators using RO4003 and LTCC substrates are presented.

\subsection{Design of the gap waveguide resonator}

Gap waveguide technology has been validated and presents many advantages for the development of $\mathrm{mm}$-wave devices. In this thesis, we focus on the characterization of the electromagnetic properties of fluids in the mm-wave band. Hence, taking advantage of the low loss of the gap waveguide, resonator based on this technology has been performed. In this section, firstly, the basic gap waveguide model will be presented and then, based on this model, the methodology to create the resonator will be described.

\subsubsection{Gap waveguide model}

The geometry of the proposed structure is shown in Figure 5.1. The chosen LTCC material is ESL41110, with $\varepsilon_{\mathrm{r}}=4.2$ and loss tangent $\tan \delta=0.004$ (values proportioned by the manufacturer). The LTCC layer thickness after firing is 75 $\mu \mathrm{m}$. The device is composed of eight LTCC layers including the via holes and mushrooms to create a high impedance surface. Other design parameters are described in Table 5.1.

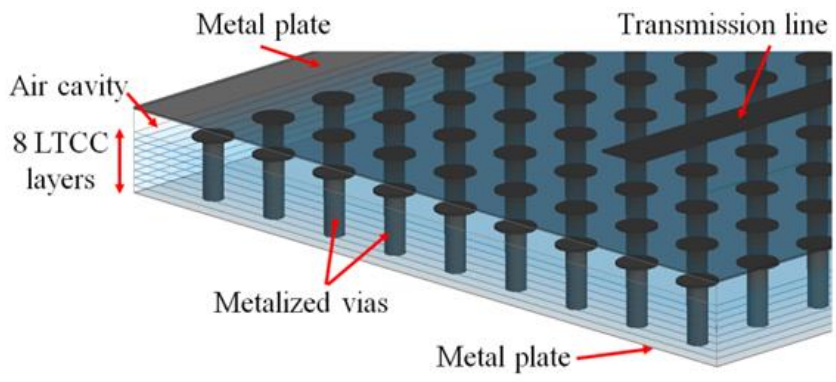

Figure 5.1. Gap waveguide geometry with LTCC. 
Table 5.1. Design parameters of the gap waveguide model with LTCC.

\begin{tabular}{|ccc|}
\hline \multicolumn{3}{|c|}{ Gap waveguide design parameters } \\
\hline Pin diameter & $\mathrm{d}$ & $200 \mu \mathrm{m}$ \\
\hline Spacing of the pins & $\mathrm{p}$ & $800 \mu \mathrm{m}$ \\
\hline Dielectric & LTCC tape: & $\varepsilon \mathrm{r}=4.2$, tan $\delta=0.004$ \\
& Ferro A6-M & Layer thickness $=75 \mu \mathrm{m}$ \\
\hline Pin height & $\mathrm{hp}$ & $750 \mu \mathrm{m}(10 \mathrm{LTCC}$ layers $)$ \\
\hline Width of the strip & wridge & $0.5 \mathrm{~mm}$ \\
\hline Air gap height & hgap & $225 \mathrm{um}$ (3 LTCC layers) \\
\hline
\end{tabular}

The computed dispersion diagram created by the surface of the periodic mushrooms is shown in Figure 5.2-a. These dispersion diagrams have been obtained by using the Eigenmode solver in HFSS. We see that the HIS stops the propagation between $42 \mathrm{GHz}$ to $75 \mathrm{GHz}$. In the Figure $5.2-\mathrm{b}$, the dispersion diagram including the ridge or strip where the propagation takes places is represented. As expected, the stop-band created by the HIS corresponds to 45 $\mathrm{GHz}$ up to $75 \mathrm{GHz}$, and the quasi-TEM mode propagated in this region is shown. Out of this band, a multimodal configuration is observed.

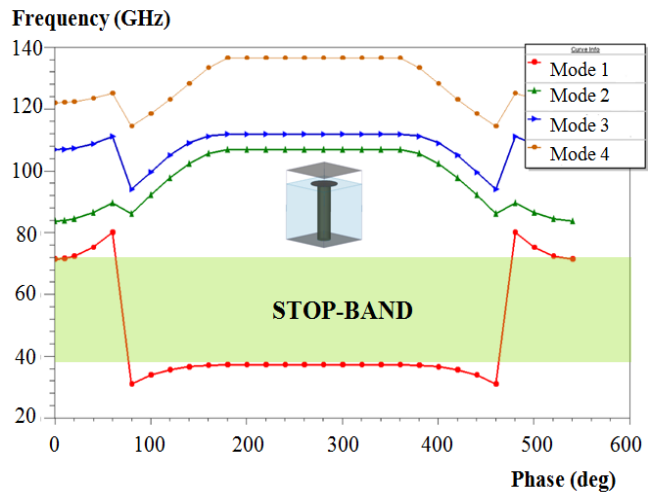

a)

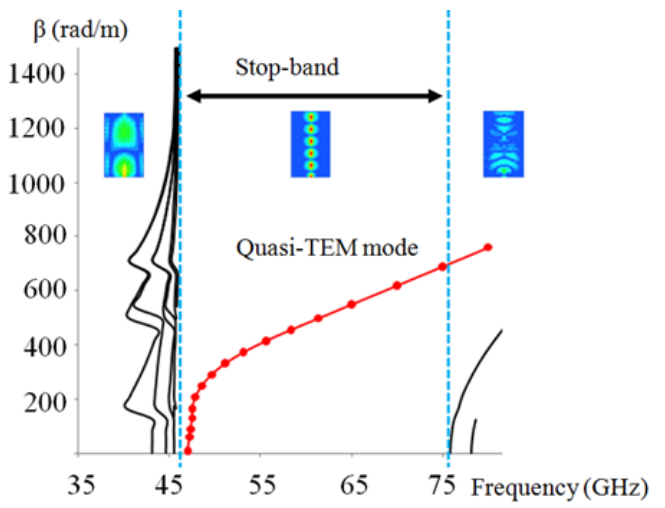

b)

Figure 5.2. Computed dispersion diagrams: stop-band created by the mushroom surface (HIS) and dispersion diagram of all the structure with the quasi-TEM mode propagated, respectively.

Once the stop-band is defined, the transmission and reflection coefficients of the structure have been computed. This response has been obtained with the Driven Modal of HFSS by wave port excitation and is it depicted in Figure 5.3 and considering dielectric and conductor losses. Return loss are less than -20 dB in the band of interest and the transmission losses are about $0.02 \mathrm{~dB}$, considering dielectric and conductor losses. 

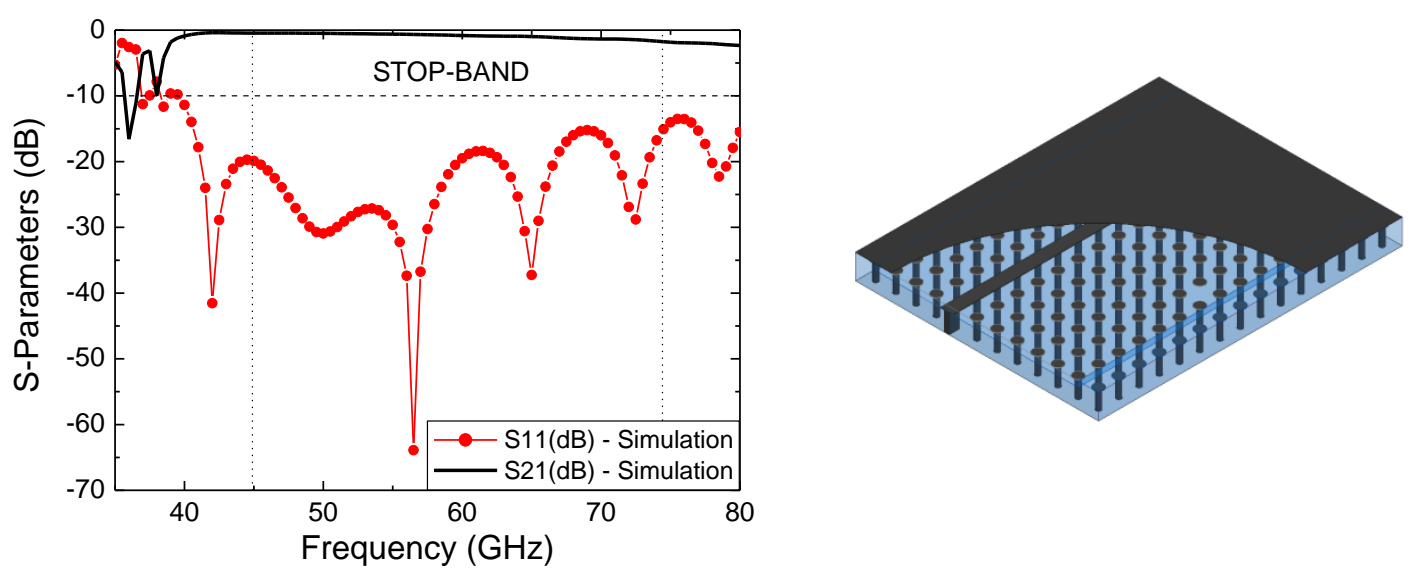

Figure 5.3. S-parameters of the gap waveguide topology.

\subsubsection{Gap waveguide resonator}

The proposed sensor uses a transmission line of the gap waveguide associated to a quarter wavelength resonator open-ended stub (Figure 5.4). The stub has been designed to get a resonant frequency in the air at $59 \mathrm{GHz}$. The mushroom surface creates the stop-band defined previously and it allows concentrating as much as possible the electromagnetic field between the transmission line and the upper metal plate.
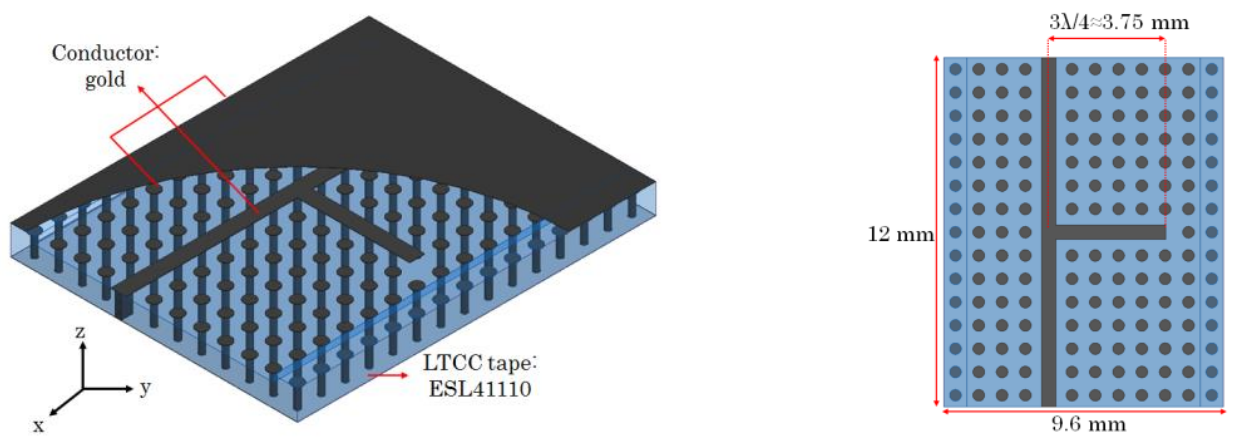

Figure 5.4. Gap waveguide resonator.

With this purpose, we present a transmission line resonator based on gap waveguide. At first, the stub length has been designed at $\lambda_{0} / 4$ that corresponds to $1.25 \mathrm{~mm}$. However, this length is not enough because the introduction of the fluid channel can perturb the E-field distributed between the strip and the upper metal plate. For this reason, a $\lambda_{0} / 2$ length was added to the stub to separate the 
maximum possible the fluid channel with the strip obtaining the same resonant frequency.

The front view of the gap waveguide with and without the LTCC wall is depicted in Figure 5.5. The total length of the stub $3 \mathrm{~N} / 4$ is $3.75 \mathrm{~mm}$. Other parameters as via height, distance between vias, diameter, etc. are the same described in Table 5.1. To create the fluid channel, it is necessary to introduce a small wall to delimitate the air cavity of the gap waveguide where the propagation takes place and the microfluidic channel. This wall is incorporated in the LTCC air cavity and has a $0.5 \mathrm{~mm}$ width (Figure 5.5).

The principal advantage of this proposed sensor is that the interaction takes place directly with the fluid under test.
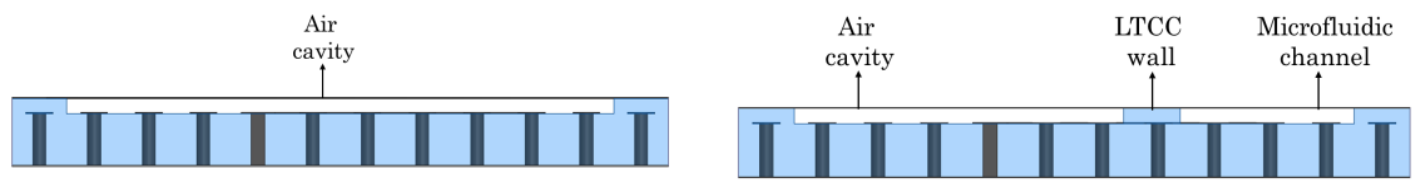

Figure 5.5. Front view of the gap waveguide resonator: Without and with the LTCC wall to separate the air cavity from the microfluidic channel.

By introducing the LTCC wall, stub might be resized in order to obtain the resonance at the same frequency, because the medium where the E-field is propagated is inhomogeneous (Figure 5.6). The optimized dimensions of the stub length are represented in Figure 5.7.

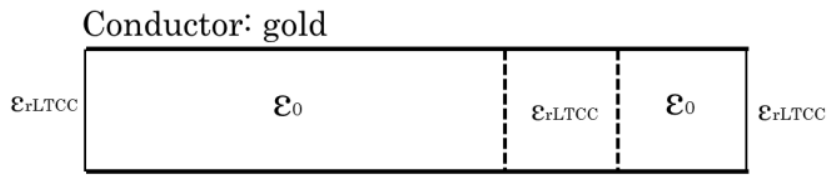

Dielectric: ESL41110 عrLTCC

Figure 5.6. Material properties and boundary condition in the gap zone between the pins and the upper metal plate. 


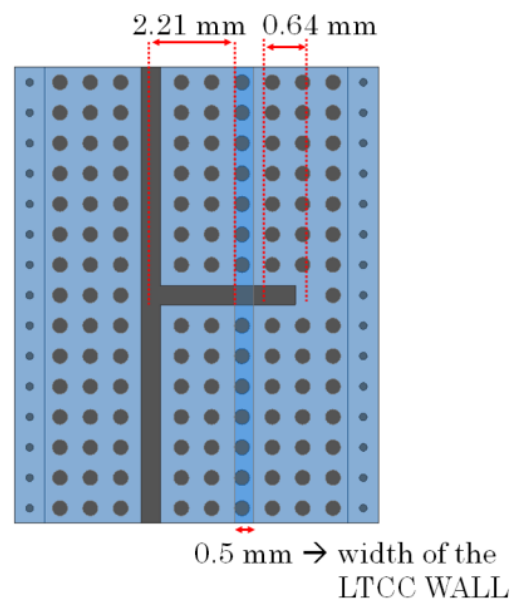

Figure 5.7. Gap waveguide resonator with LTCC wall separating the air cavity from the microfluidic channel.

In Figure 5.8, transmission and reflection coefficients of the gap waveguide resonator including the LTCC wall are depicted. As we can observe, the resonant frequency is at $59 \mathrm{GHz}$. At $73.5 \mathrm{GHz}$, another resonance is shown. This resonance corresponds to the upper limit of the stop-band created by the HIS, hence the other modes begin to propagate and perturb the resonator.

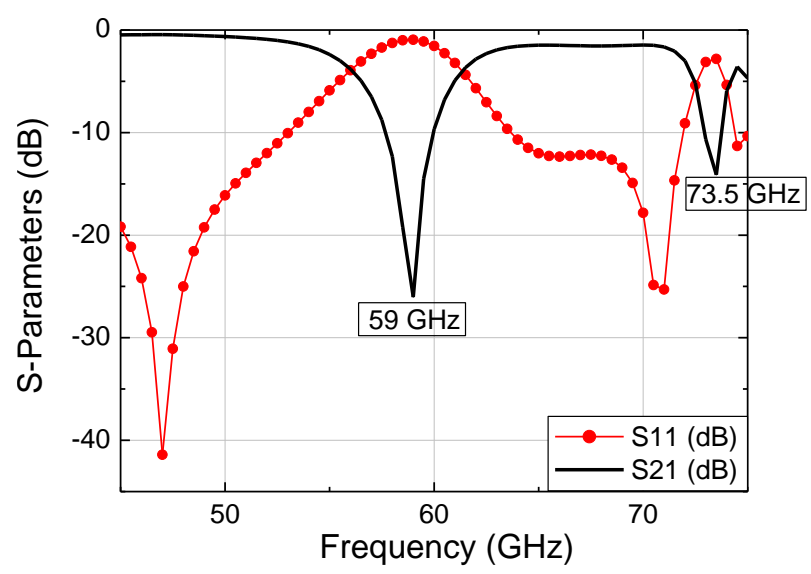

Figure 5.8. S-parameters of the gap waveguide resonator including the LTCC wall to separate the air cavity from the microfluidic channel.

The E-field distribution in the air cavities and LTCC wall is represented in Figure 5.9. We can see that at $50 \mathrm{GHz}$ (inside the stop-band), the E-field is concentrated between the strip and the upper metal plate. Then, at $59 \mathrm{GHz}$ the resonance is produced, we can observe that at this frequency all the propagation 
is rejected by the stub $3 \mathrm{~N} / 4$. Next analyzed frequency is $70 \mathrm{GHz}$, always inside the stop-band, however this frequency is placed at the end of the stop-band, hence the E-field begins to be dispersive with the vias located next to the strip. Finally, the frequency $73.5 \mathrm{GHz}$ is outside the stop-band, as it can be seen, E-field is no longer confined between the strip and the upper plate but it is dispersed over all the mushrooms surface. This dispersive behavior creates the resonance depicted in Figure 5.8.

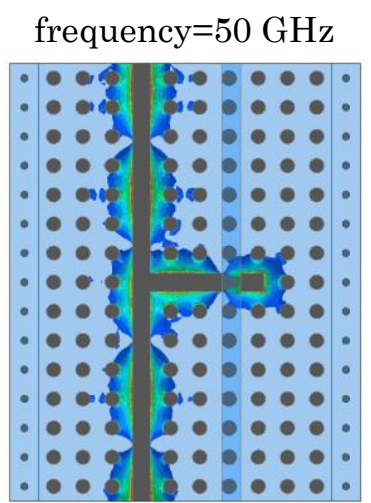

frequency $=70 \mathrm{GHz}$

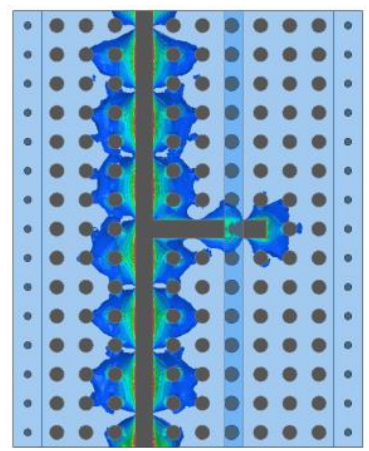

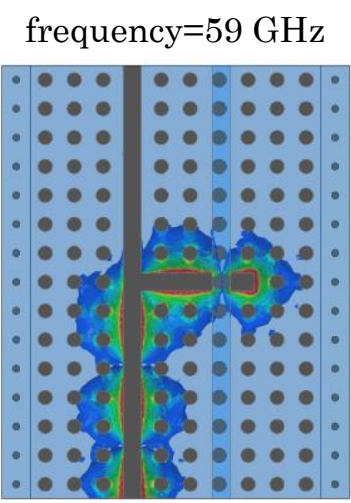

frequency $=73.5 \mathrm{GHz}$

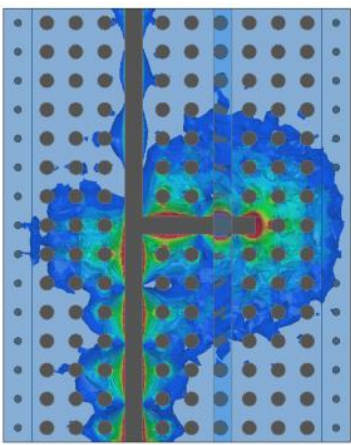

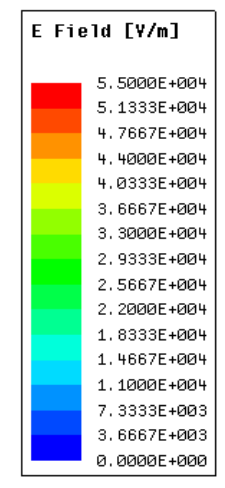

,

Figure 5.9. E-field distribution in the air gap cavity, LTCC wall and air channel.

\subsection{Comparison between gap waveguide and SIC for fluid detection}

Gap waveguide technology is presented in this chapter as a good candidate for fluid permittivity characterization. To prove the interest of our solution, a comparison with other published solutions has been done. In [80] a Substrate Integrated Cavity (SIC) using LTCC technology is presented for fluidic detection at $60 \mathrm{GHz}$. The scheme is presented in Figure 5.10. The advantages of the proposed SIC sensor are the compact size (due to dimensions and use of LTCC) 
and the fact that the liquid can flow underneath the cavity and does not have to enter it. However, the sensitivity of this sensor can be improved if the fluidic channel is inserted in the structure itself.

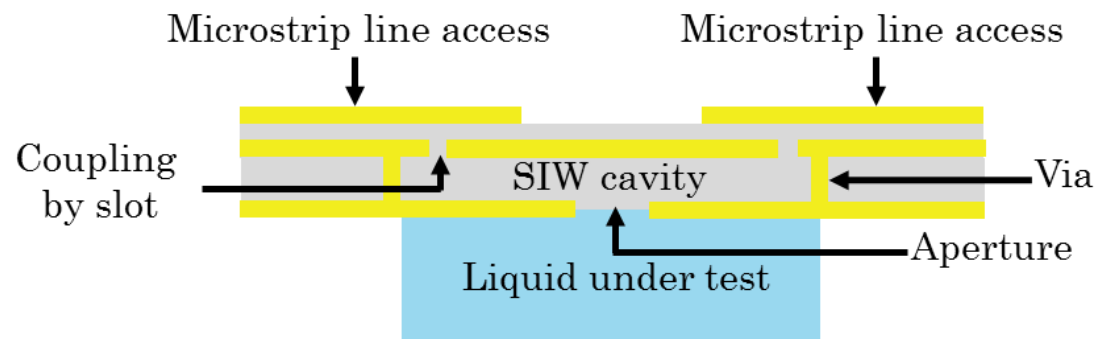

Figure 5.10. Cavity resonator based on Substrate Integrated Cavity at $60 \mathrm{GHz}$ [80].

As it has been explained in section 5.1.2 a resonator based on gap waveguide technology is proposed. In our sensor, the interaction takes places directly between the resonator and the fluidic channel created in the air cavity of the gap waveguide (Figure 5.11 and Figure 5.12). With our design, we can extract the dielectric properties of the fluid by the electromagnetic characteristics of the resonator.

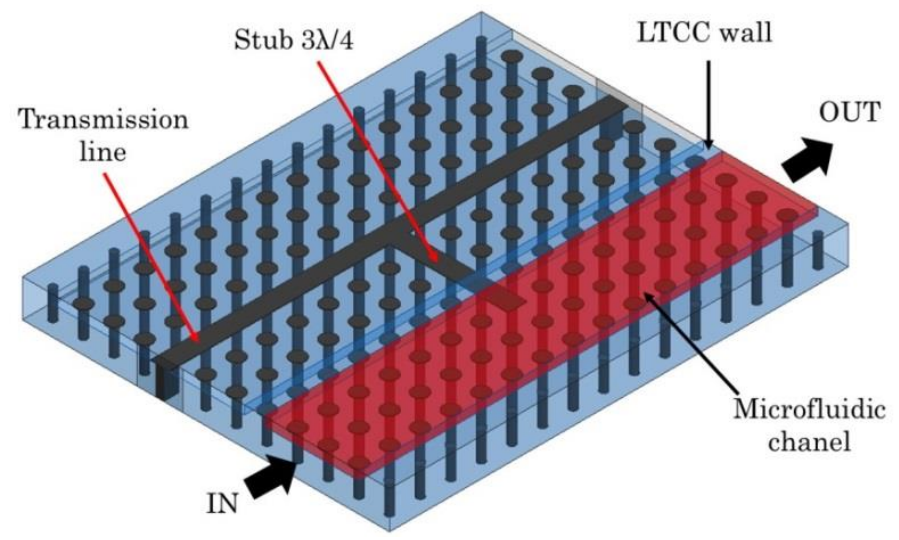

Figure 5.11. 3D view of the gap waveguide stub resonator for fluid detection.

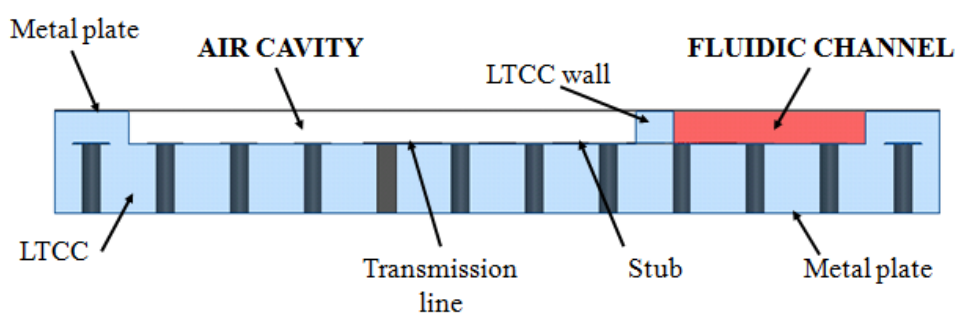

Figure 5.12. Front view of the gap waveguide resonator. 
In [80], S-parameters of the cavity filled with water, methanol and also the empty case for reference, are presented and we summarize in Figure 5.13. The empty cavity has a $58.6 \mathrm{GHz}$ as resonant frequency. When the cavity is filled with methanol $(\varepsilon \mathrm{r} \approx 7)$ the resonant frequency is $58.15 \mathrm{GHz}$ and with water $(\varepsilon \mathrm{r} \approx 13)$ is $57.75 \mathrm{GHz}$.

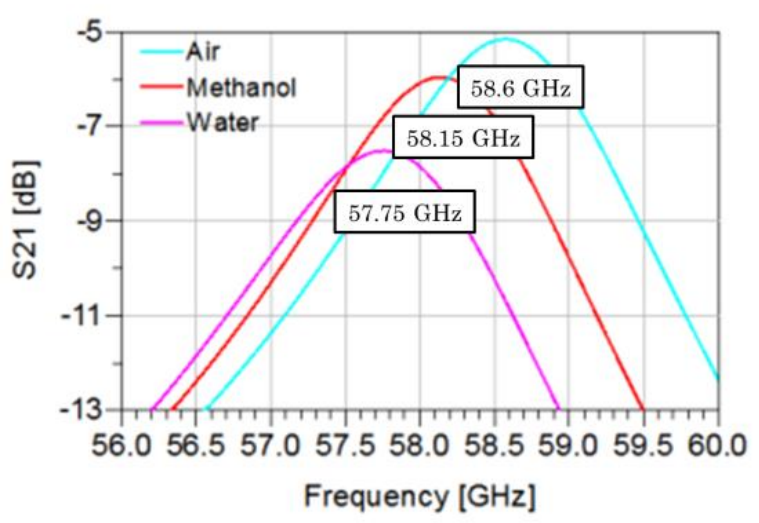

Figure 5.13. Computed results of the SIC sensor presented in [6] of the cavity loaded with air, methanol and water.

To test our structure, the material properties can be added to HFSS by giving its frequency dependent dielectric constant and loss tangent. The materials under test are methanol, water and the empty cavity (air). Results concerning the gap waveguide resonator with the fluidic empty cavity are depicted in Figure 5.14-a. The resonance frequency is at $59 \mathrm{GHz}$ as it has been shown in Section 5.1.2. In Figure 5.14-b and Figure 5.14-c, the transmission and reflection coefficients of the cavity filled with methanol and water, respectively are represented. The filled cavity with methanol resonates at $49 \mathrm{GHz}$ and at $45.5 \mathrm{GHz}$ with water.

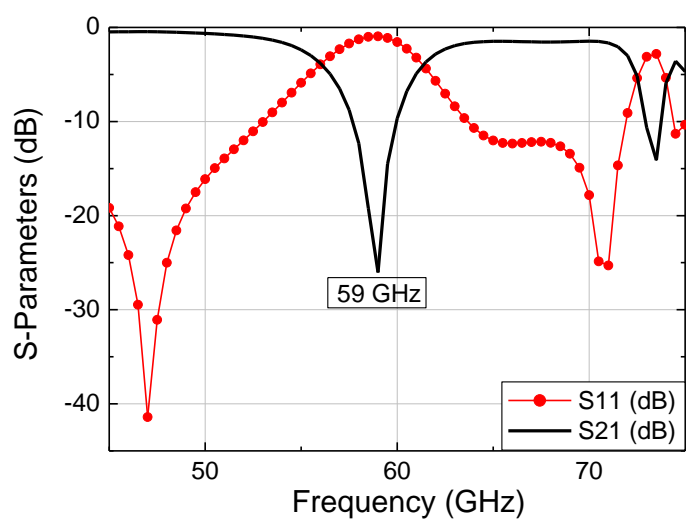

a) Without fluid 


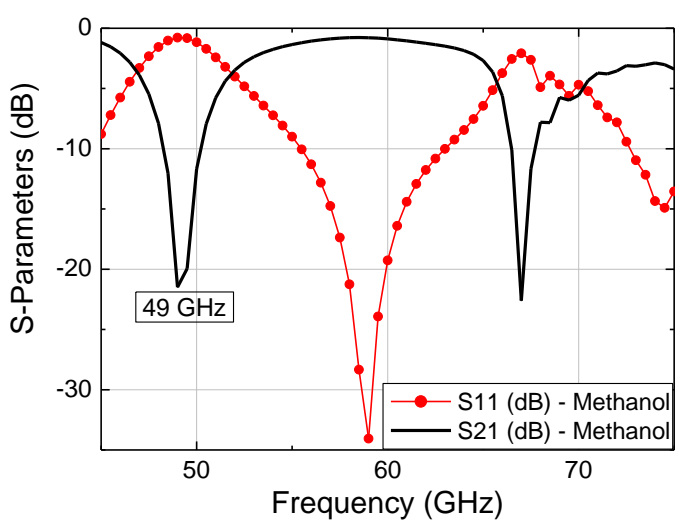

b) With methanol $\varepsilon r=7$

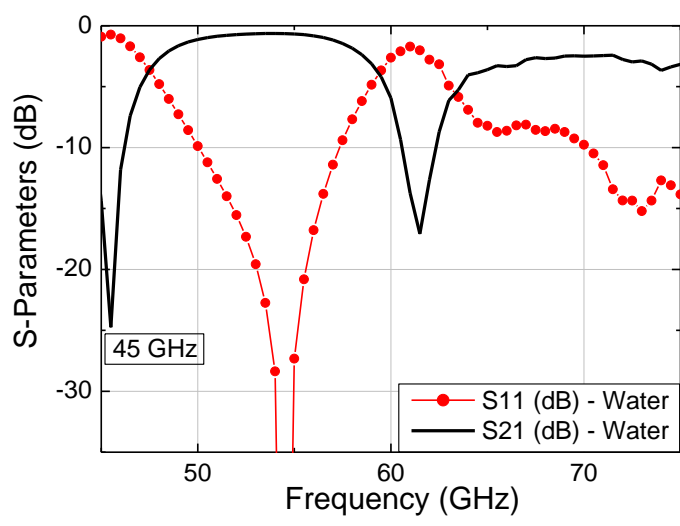

c) With water $\varepsilon r=13$

Figure 5.14. Transmission and reflection coefficient for fluidic cavity filled with: air, $\varepsilon r=7$ and $\varepsilon r=13$.

To summarize the results, in Figure 5.15, only the transmission coefficients of the three considered cases: empty cavity, with methanol and with water are represented. Moreover, values of the resonant frequency with gap waveguide and with SIC are depicted in Table 5.2.

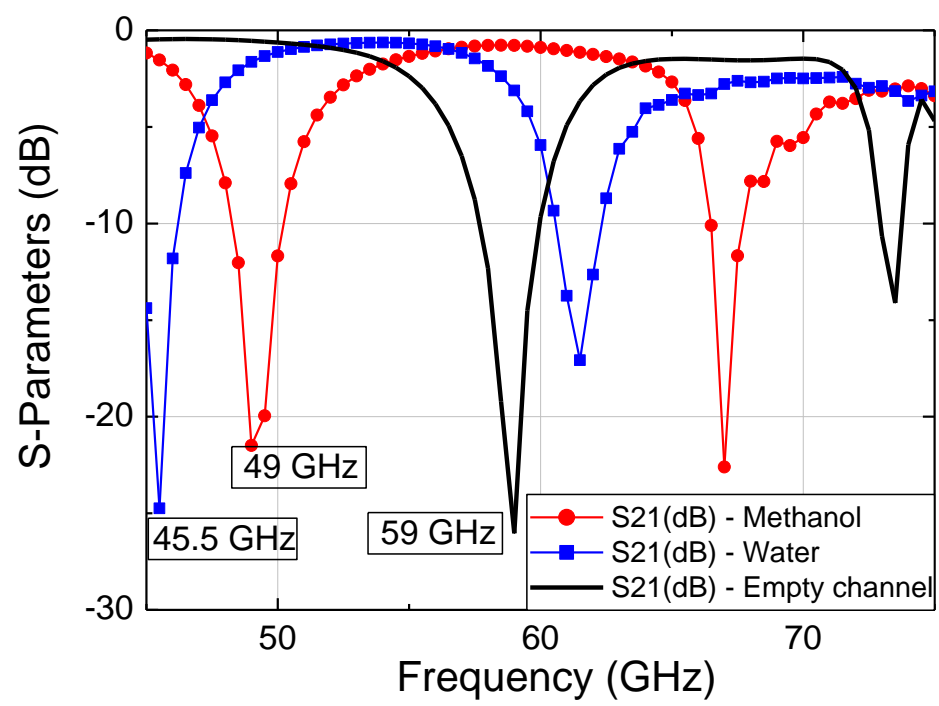

Figure 5.15. Transmission coefficient for the fluidic cavity with: air, $\varepsilon r=7$ and $\varepsilon r=13$. 
Table 5.2. Computer resonant frequency and comparison between SIC and gap waveguide.

\begin{tabular}{|c|ccc|}
\hline Guided structure & \multicolumn{3}{|c|}{ Resonant frequency (GHz) } \\
\cline { 2 - 4 } & Water & Methanol & Air \\
\hline SIC & 57.75 & 58.15 & 58.6 \\
\hline Gap waveguide & 45.5 & 49 & 59 \\
\hline
\end{tabular}

Results show the impact of the fluid properties in the electromagnetic response of the structure with a variation of the resonant frequency. Compared to the results obtained in [80], the frequency shift between different compositions, with the proposed structure is three times higher than those achieved using Substrate Integrated Cavity. The higher value of sensitivity of the gap waveguide is due to the interaction between the fluid and the electromagnetic propagation takes place in the maximum level of the E-field. However using other classical structures like printed planar transmission lines or SIW, the fluid detection is by means of apertures and external cavities. In conclusion, allows improving the level of sensitivity in terms of variation of the resonant frequency in the $\mathrm{mm}^{-}$wave band.

We assume dielectric loss of the fluids by including the $\tan \delta$ value of the dielectric. The electric loss tangent can be defined as (5.1).

$$
\tan \delta=\frac{\varepsilon^{\prime \prime}}{\varepsilon^{\prime}}
$$

The values of the dielectric constant and losses for methanol and water at 60 $\mathrm{GHz}$ are depicted in Table 5.3. Then, we compute the influence in the transmission response and they are represented in Figure 5.16. As it can be noticed in this figure, the introduction of the methanol and water produces a shift in the resonant frequency of $5.5 \mathrm{GHz}$ and $10 \mathrm{GHz}$.

As conclusion, we can say that detection is less sensitive when losses are introduced even always more sensitive than in the case of Substrate Integrated Cavity.

Table 5.3. Dielectric properties of the fluids under test at $60 \mathrm{GHz}$.

\begin{tabular}{|cccc|}
\hline & $\boldsymbol{\varepsilon r} \mathbf{r}^{\prime}$ & $\boldsymbol{\varepsilon r} \mathbf{r}^{\prime}$ & $\tan \boldsymbol{\delta}$ \\
\hline Methanol & 7 & -1.5 & 0.214 \\
Water & 13 & -24 & 1.846 \\
\hline
\end{tabular}




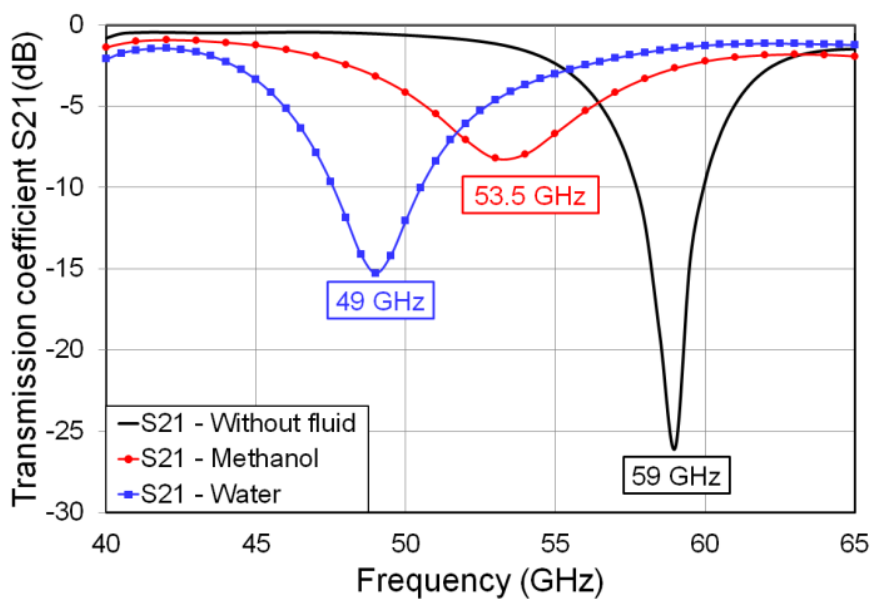

Figure 5.16. Transmission coefficient for the fluidic channel: empty, filled with methanol and with water.

We compare the three resonant frequencies obtained with gap waveguide and SIC as a function of permittivity (including fluid losses). Results are shown in Figure 5.17. As can be seen, with SIC there is hardly any variation of the resonant frequency value. Nevertheless, the variation of the resonant frequency is more abrupt with gap waveguide. Hence, we can conclude that the sensitivity variation is more significant with gap waveguide, which allows an easiest detection of electromagnetic changes due to material properties.

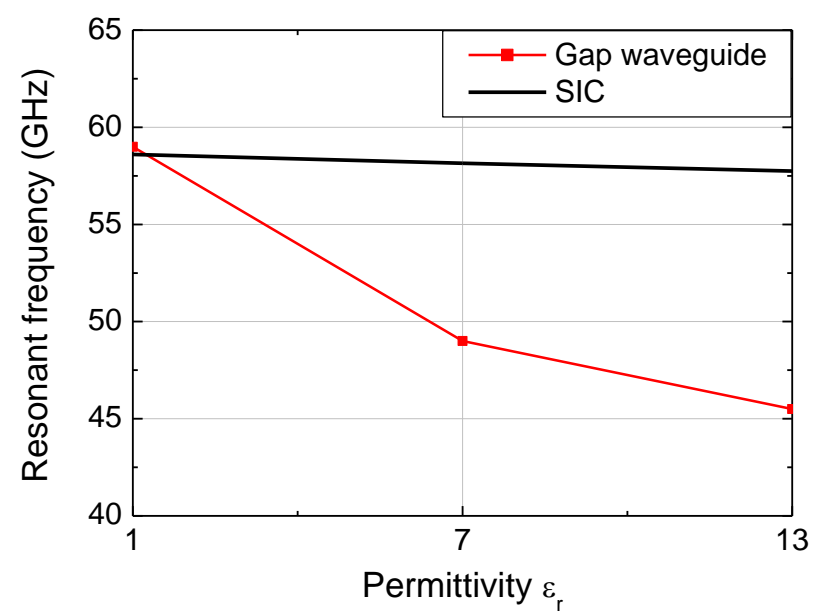

Figure 5.17. Resonant frequency in function of the permittivity for the 3 cases: empty channel, with methanol and water. 


\subsection{Manufacturing and measurements of the gap waveguide resonator with PCB and LTCC}

The resonator-based gap waveguide was initially manufactured with a classical substrate like RO4003 ( $\varepsilon r=3.55$ and $\tan \delta=0.0027)$ and then with LTCC ESL41110 ( $\varepsilon r=4.2$ and $\tan \delta=0.004)$.

\subsubsection{Gap waveguide with Printed Circuit Board (PCB)}

The manufactured prototype with RO4003 has been presented in Chapter 4 . We focus here on the gap waveguide-based resonators. As depicted in Figure 5.18, there are three stub resonators namely GW3, GW4 and GW6. In the case of the prototype GW3, measurements have not been possible due to etching problems of the transition. Therefore, we are going to analyze the prototype GW4 and GW6.

To recall the device studied, the transition in Figure 5.19, the manufactured gap waveguide resonator, the ground plane with the $\mathrm{H}$-shape apertures and the coplanar-to-microstrip transition are shown.

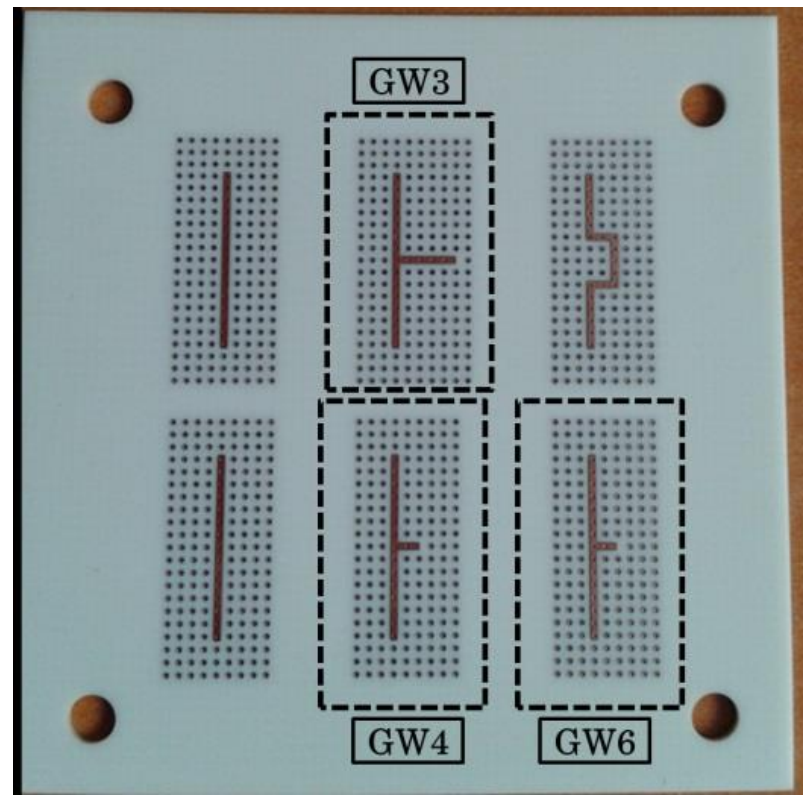

Figure 5.18. Six gap waveguide prototypes using RO4003 as substrate. 

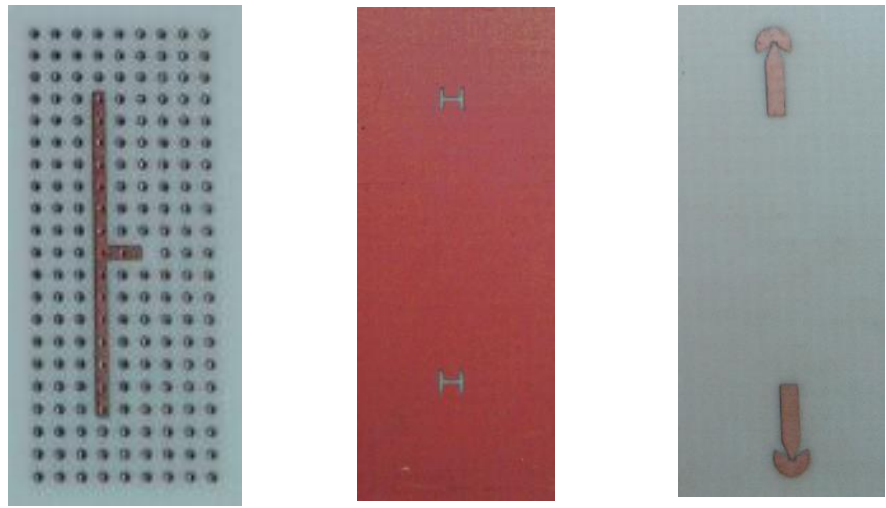

Figure 5.19. Gap waveguide resonator, ground plane with $\mathrm{H}$-shape apertures and coplanar to microstrip transition manufactured with RO4003

\section{GW4: $\lambda_{0} / 4$ Gap Waveguide resonator}

GW4 consists of $\lambda_{0} / 4$ stub resonator, being the designed stub length $1.65 \mathrm{~mm}$ to have a simulated resonant frequency at $42.2 \mathrm{GHz}$, as we can see in Figure 5.20. This resonant frequency is produced in the [40.7-74.8] $\mathrm{GHz}$ stop-band region created by the gap waveguide. Measurements of the prototype are included in Figure 5.20. A good correspondence between measurements and simulations is shown.

The small difference between measurements and simulations can be explained as the stacked PCB layers (gap waveguide, cavity layer and transition) introduce a higher thickness for the air cavity than the expected value.

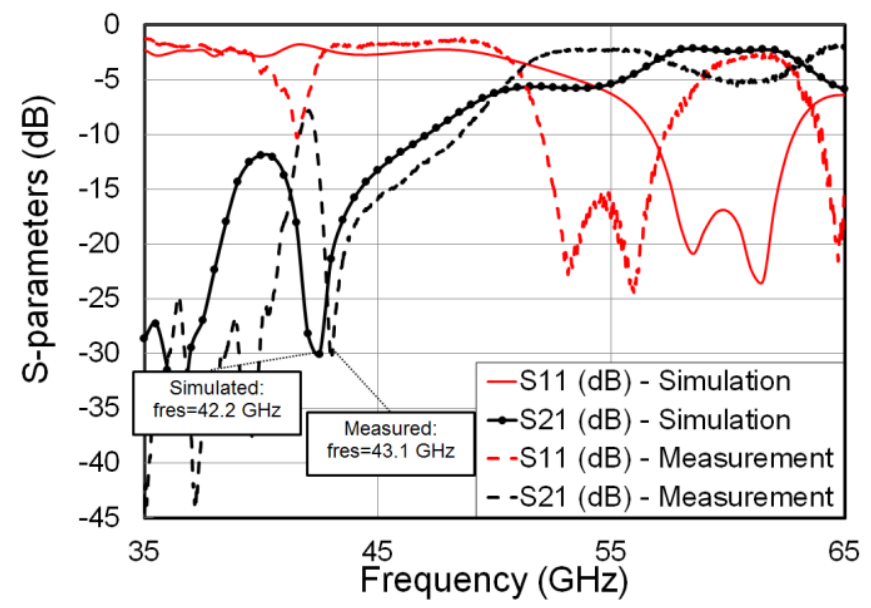

Figure 5.20. Computed and measured results for the gap waveguide resonator GW4. 


\section{GW6: $\sim \lambda_{0} / 4$ gap waveguide resonator}

GW6 corresponds to the second gap waveguide resonator with a stub length of $1.55 \mathrm{~mm}$ (computed resonant frequency $44.9 \mathrm{GHz}$ ). The computed and measured results are depicted in Figure 5.21. As it is seen, also in this case, measurements are in agreement with the computed results at the resonant frequency. The shift produced in the reflection coefficient response can be explained by the increase of the air gap thickness of the PCB stacking.

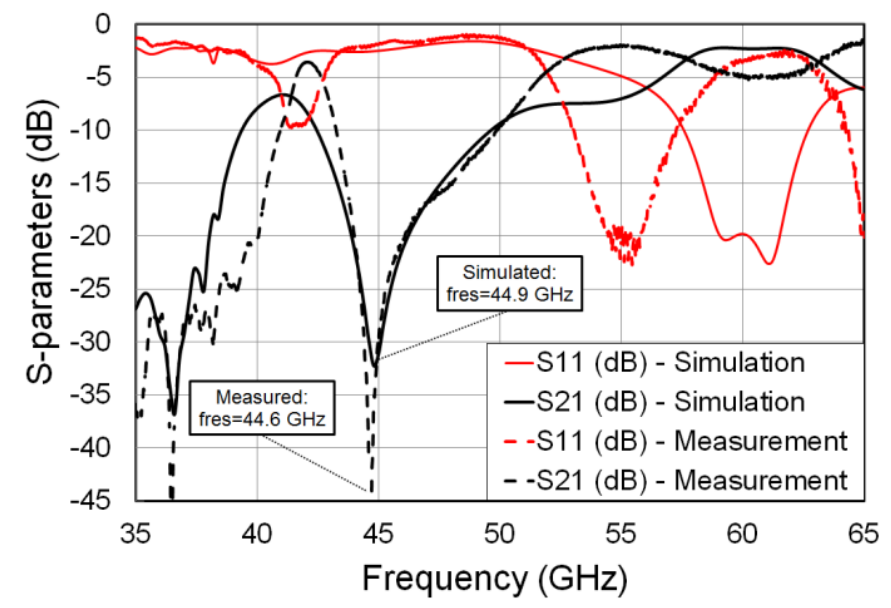

Figure 5.21. Computed and measured results for the gap waveguide resonator GW6.

In conclusion, manufactured gap waveguide resonators with R04003 are validated with a good agreement in relation to the computed results. Nevertheless, the increase in gap height of the gap waveguide structure, leads to an impedance mismatch with the coplanar-to-microstrip transition. An increase of insertion losses is produced and also a shift in the $\mathrm{S} 11$ response.

\subsubsection{Gap waveguide with LTCC}

The prototype was manufactured using LTCC with ESL41110 tape. In this section, the two resonators based on gap waveguide technology will be discussed. In Chapter 4, the LTCC manufactured prototype has been presented (Figure 5.22 ) and some technological problems of this prototype have been explained. 


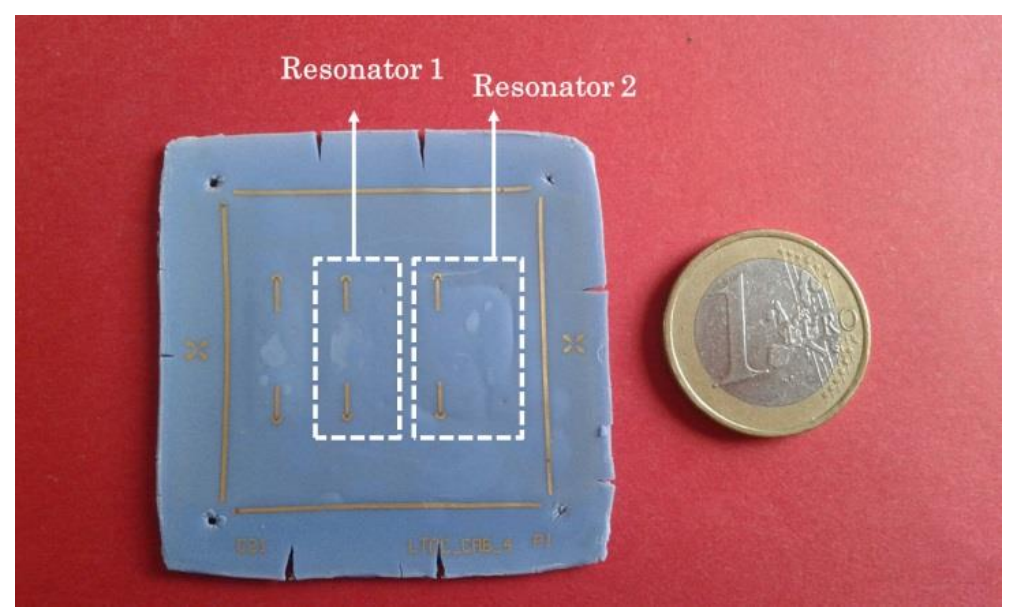

Figure 5.22. Manufactured LTCC prototype.

In Figure 5.23, several steps of the manufacturing process of LTCC are shown. In the first case we can see the stacking process. In this figure, the three prototypes are represented. In this chapter we are focused in the two resonators. The last layer represented corresponds to the last LTCC layer to create cavities. For both resonators, we distinguish two parts: one cavity for the E-field propagation and another for the introduction of the fluid. Figure in the righthand side shows the introduction of the sacrificial volume material (SVM) before lamination. SVM material is ESL49000 tape that prevents deformation during high pressure lamination and contains high purity carbon which burns out during the firing process.
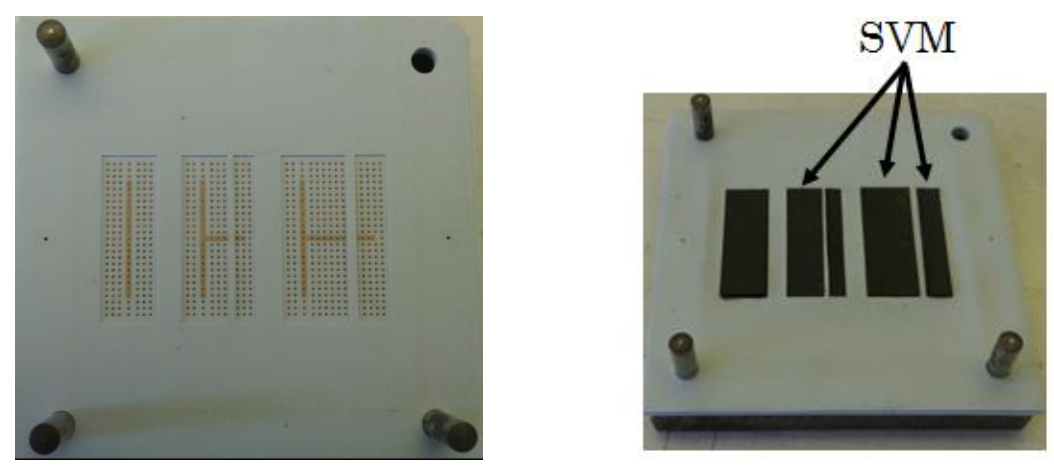

Figure 5.23. Pictures of the stacking process of the LTCC prototype.

Once, the manufactured prototype has been presented, we analyze the two resonators based on gap waveguide concept. The HIS created by the mushroom surface creates a stop-band between 41.3 and $62.9 \mathrm{GHz}$. The $\mathrm{CPW}$-to gap 
waveguide transition presents an operating frequency band between 46 and 59 $\mathrm{GHz}$ with return loss $<-10 \mathrm{~dB}$.

\section{RESONATOR 1: 3N/4 stub length}

This resonator consists on a $3 \mathrm{~N} / 4$ long stub at $55 \mathrm{GHz}$ resonant frequency based on gap waveguide technology. $3-\mathrm{D}$ view and dimensions are depicted in Figure 5.24 .
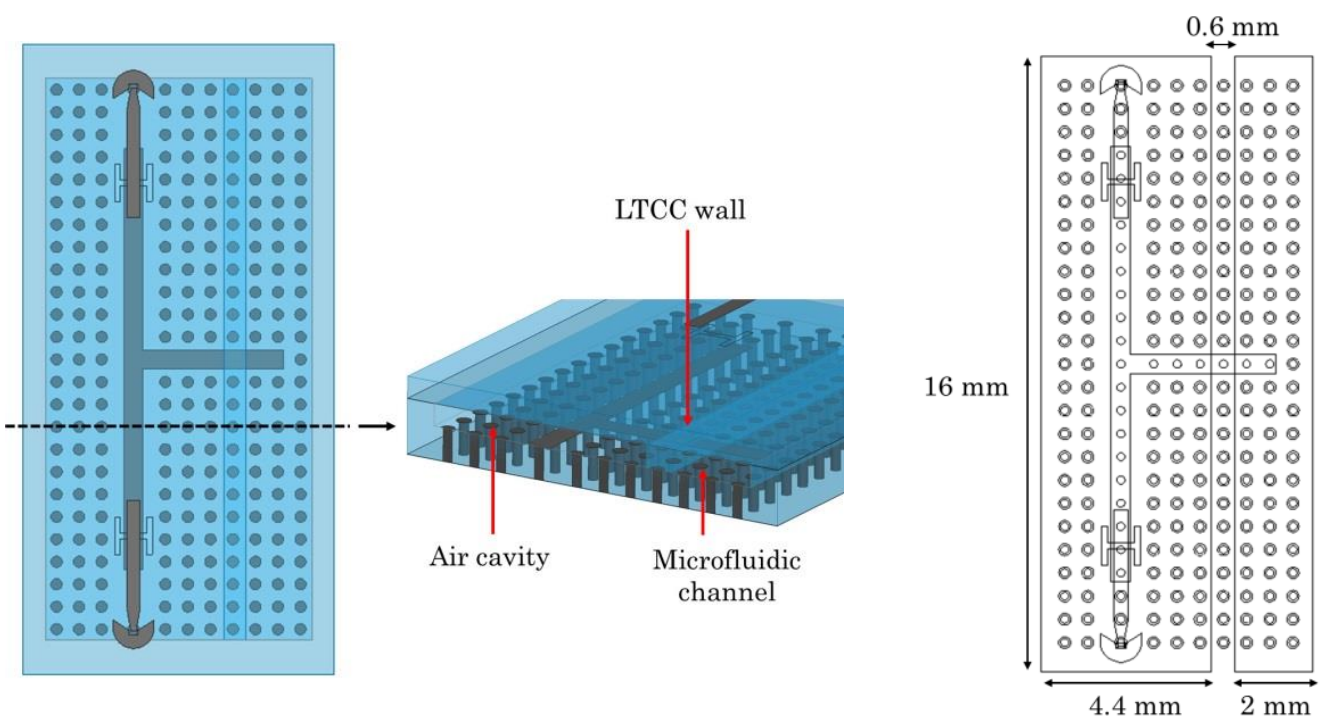

Figure 5.24. Top view of the 3X/4 resonator using LTCC and cavity dimensions.

Considering gold conductor and ESL41110 tape as dielectric with tan $\delta=0.004$, the computed and measured results are represented in Figure 5.25. As we can see, the measured results show that no propagation between the back to back configurations is done with a level of the transmission parameter less than -15 dB. Several complications in the manufacturing process had occurred. More specifically the metallization of the ground plane of the microstrip transition has overflowed into the cavity, hence a metallic boundary in the air gap has been created and it implies that no transmission takes place. 


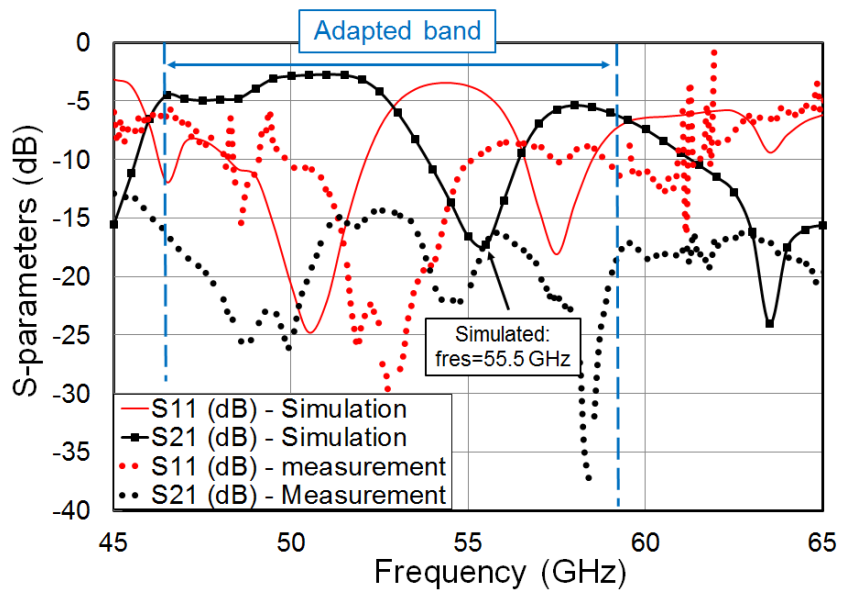

Figure 5.25. Comparison between computed and measured results for the $3 \mathrm{~N} / 4$ gap waveguide resonator using LTCC.

\section{RESONATOR 2: $5 \lambda / 4$ stub length}

This resonator has a stub length equal to $5 \lambda / 4$. This structure has been designed to have a resonant frequency at $53.75 \mathrm{GHz}$. Top view and dimensions of the cavities are depicted in Figure 5.26.
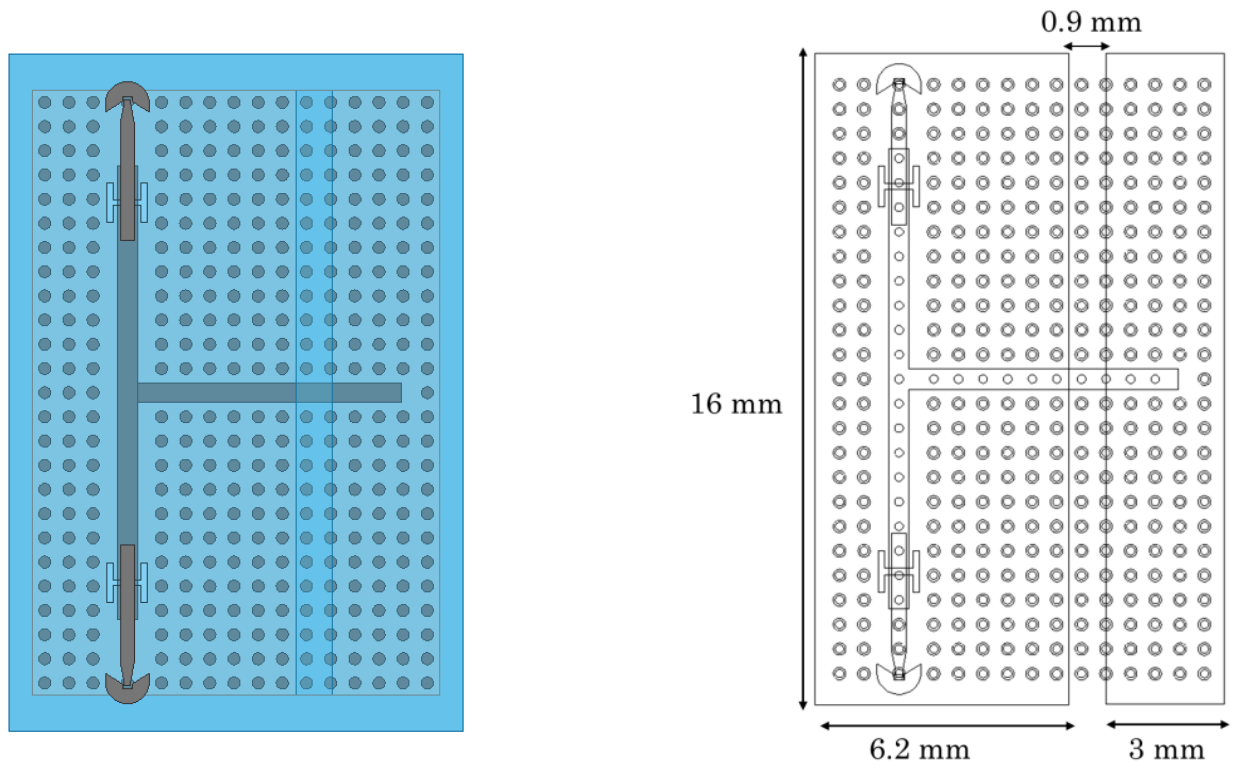

Figure 5.26. Top view of the 5X/4 resonator and cavity dimensions.

The LTCC wall has been designed with a width $=0.9 \mathrm{~mm}$ to ensure that the LTCC wall will not be deformed in the LTCC co-fired process. 
Measured results of this wall are in agreement with the design. Measured width is $0.935 \mathrm{~mm}$ (Figure 5.27).
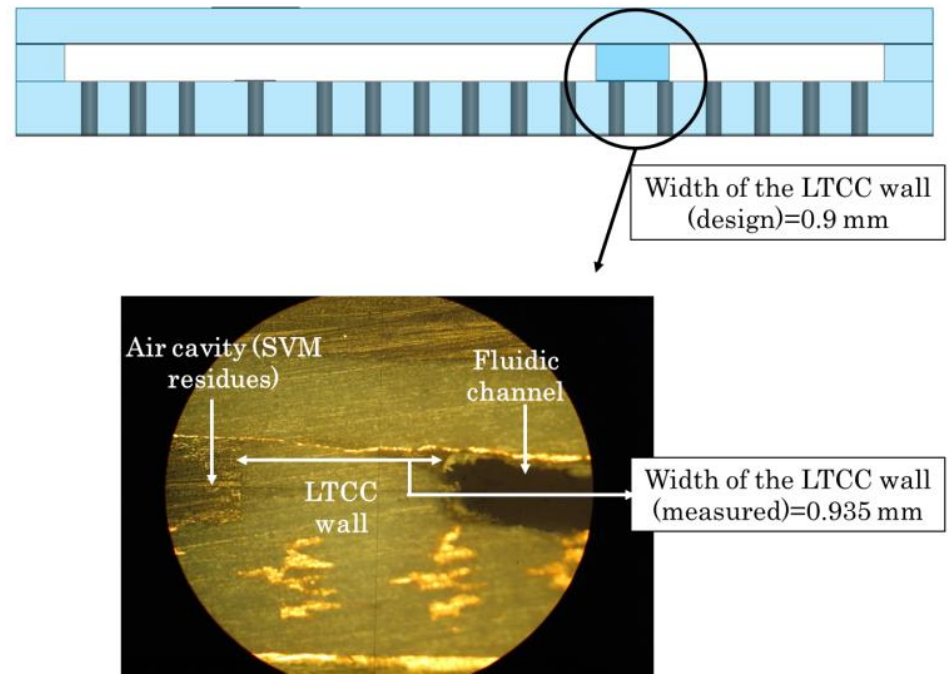

Figure 5.27. Dimensions of the LTCC wall designed and measured.

A comparison between simulation and measured results is represented in Figure 5.28. As stated above, we found the same problems as in the gap waveguide resonator $3 \mathrm{~N} / 4$. The transmission attenuation is less than $-10 \mathrm{~dB}$. Moreover, an error occurred in the manufacturing process can produce a bad touch between the coplanar transition and the coplanar probes. More precisely in the gold serigraphy of the transition as it is shown in Figure 5.29. The coplanar to microstrip transition is deformed by the cavity and the GSG probe does not touch the ground plane of the coplanar design.

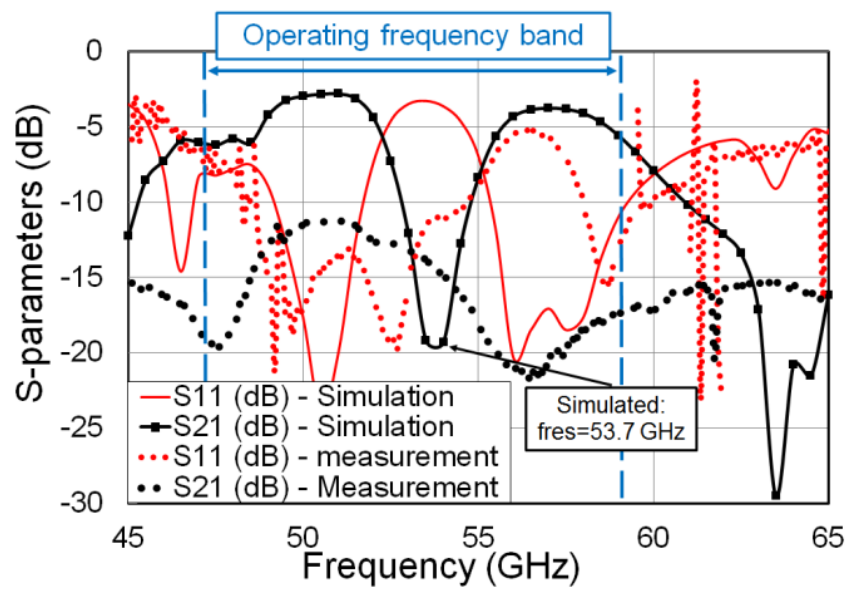

Figure 5.28. Computed and measured results for the $5 \mathrm{~N} / 4$ gap waveguide resonator. 


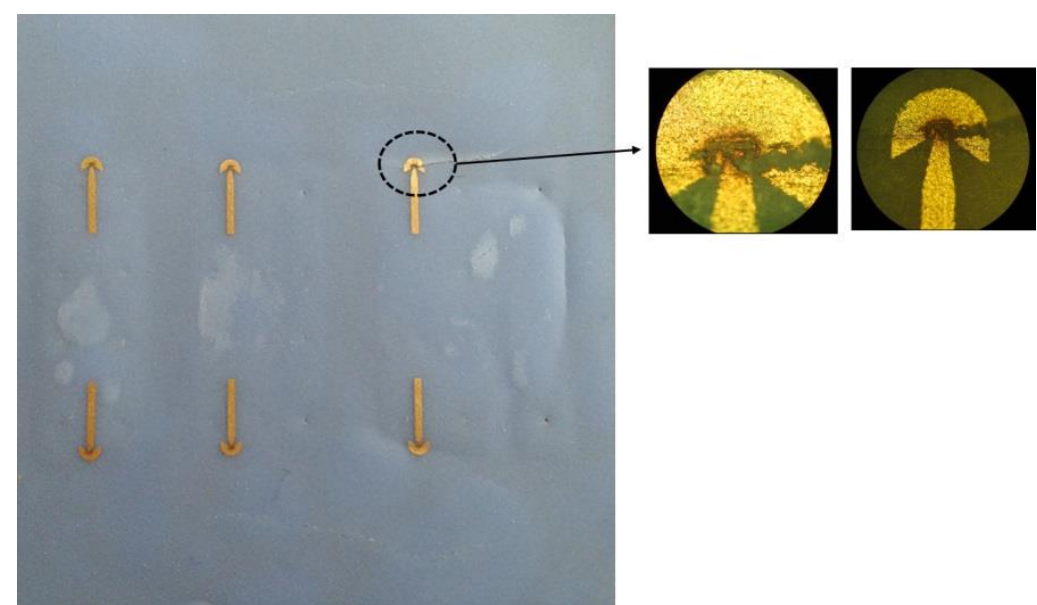

Figure 5.29. Manufactured LTCC prototype. Bad gold serigraphy in the transition 3 for the 5N/4 gap waveguide resonator. Hard to make contact with the probes used for measurements.

We can conclude of the LTCC manufacturing process of the cavity test and the gap waveguide than:

- Creation of internal cavities has been validated in terms of dimensions and deformation. However, if the metallization of the bottom LTCC layer is the top of the internal cavity, some difficulties have been observed and the gold has descended in the cavity.

- The minimum distance between two cavities (some tests have been done to find the minimum length of the LTCC wall) being $0.5 \mathrm{~mm}$ validated.

- Problems in the control of the via filling process and in the lamination process in our laboratory.

- Shift of the energy coupling aperture between the microstrip transition and the gap waveguide.

\subsection{Other resonant structures based on gap waveguide}

With the same principle of the stub gap waveguide resonator, we propose another resonator based on gap waveguide: the ring resonator. This structure has the advantage of resonances takes places in the maximum level of transmission parameters that can simplify the detection. Moreover, in terms of resonant frequencies we are more flexible for electromagnetic properties changes due its multiple resonances. 
The designed structure and the computed response are depicted in Figure 5.30.
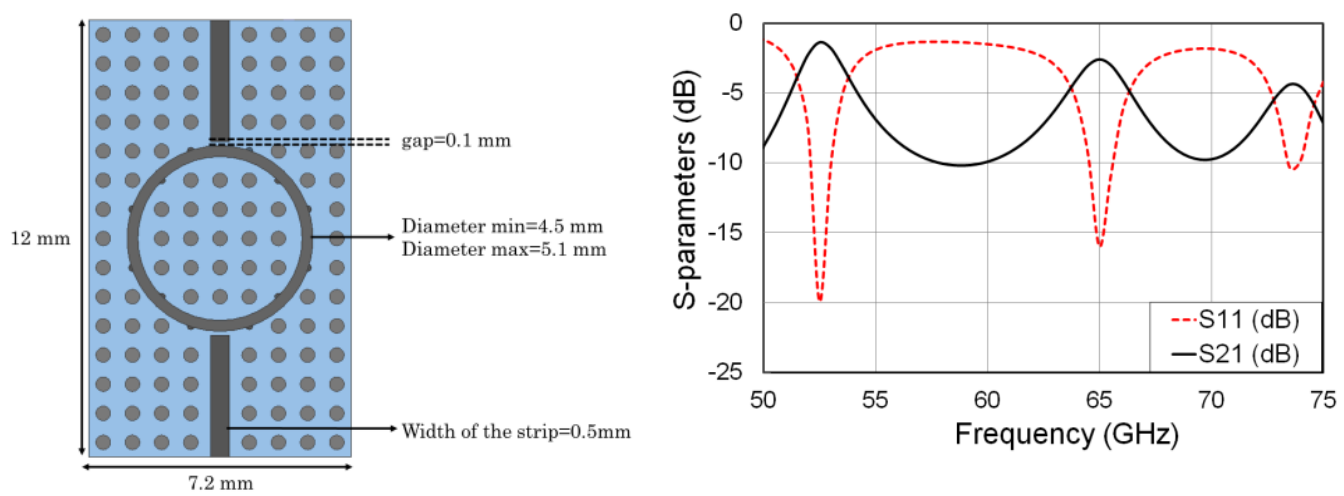

Figure 5.30. Proposed ring resonator based on gap waveguide technology and electromagnetic response.

The proposed microfluidic cavity is shown in Figure 5.31. This cavity is inserted in the centre of the ring resonator to have a maximum interaction between the fluid and the electromagnetic field.
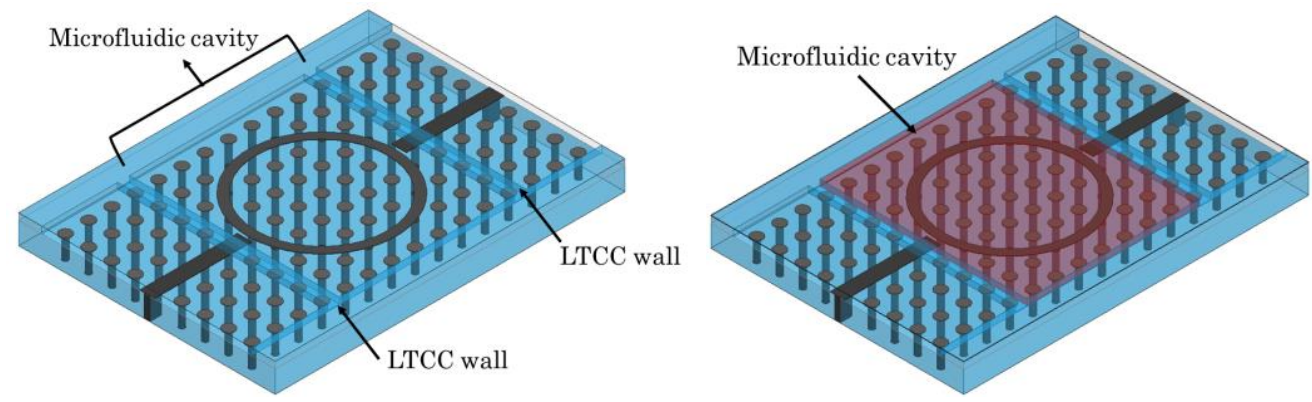

Figure 5.31. Gap waveguide ring resonator with the microfluidic cavity.

\subsection{Conclusion}

In this chapter we discussed the interest of the gap waveguide technology for the characterization of the electromagnetic properties of fluids. Firstly, the design of the quarter wavelength resonator with an open-ended stub based on the gap waveguide technology has been presented. Then, the design including the inserted channel to introduce the fluid under test has been characterized in terms of EM simulations. Once, a comparison between gap waveguide and Substrate Integrated Cavity has been performed for three different fluids. This comparison shows the impact of the electromagnetic properties of the fluid in the electromagnetic response of the structure with a variation of the resonant 
frequency. In conclusion, gap waveguide exhibits more sensitivity to changes in the fluid because the interaction takes place in the maximal concentration of the electromagnetic field, while with SIC by means of apertures and external cavities. This resonant method allows characterizing medium with a good sensitivity, for example, in diagnosis applications, to determine the concentration level of cells in a medium.

Then, the design and manufactured prototype using PCB and LTCC has been presented. The gap waveguide resonators based on PCB technology exhibits a good agreement between simulation and measurements. However, a strong dependency in the gap height has been observed. Using multilayer PCB technique, all the parts are stacked and this process can produce variations in the height of the air gap cavity. Changes in the air gap height have a considerable impact on the matching and the transmission between the transition and the gap waveguide. To overcome these problems, LTCC is presented as an appropriate technology with the aim to create in one step the entire module including the via surface, the air cavity and the transition. This prototype has been designed and presented, where some technological problems have taken place that they were defined in Chapter 4. 


\section{FINAL CONCLUSIONS AND FUTURE LINES}

\section{Final conclusions}

Microfluidic is a field of big expansion nowadays due to the improvement made in the device miniaturization. This Ph.D. dissertation presents the results trying to improve the sensibility of the sensors in the millimeter-wave band. It has been demonstrated that the $\mathrm{mm}^{-w}$ ave offers new possibilities in the fluid characterization. During the Ph.D. dissertation a contribution in the design of a new guiding structure and the manufacturing using PCB and LTCC techniques has been done for the design of the final sensor to provide higher sensibility in the detection.

After an introduction where the importance of the millimeter-wave band was presented and the current manufacturing techniques in microfluidic applications were described, an analysis of the guiding structures capable to integrate microfluidic channels and to operate in the mm-wave band was presented. The desired guiding structured aims to optimize the detection of the fluid in the maximum electromagnetic field. . In this context, gap waveguide technology is presented as a good candidate to overcome most of the issues of the conventional guiding structures. Gap waveguide structures are compatible with PCB and LTCC techniques, being the latter very suitable for microfluidic applications due the biocompatibility with the fluid properties. This chapter serves as a starting point to understand the work that has been during this Ph.D. dissertation. 
In the second chapter, the design and the principle of operation of the gap waveguide was presented. We introduce the design of these structures embedded in LTCC substrate. The design considerations are explained and a losses study of this topology is undertaken.

Chapter 3 was focused on the advantages of the gap waveguide technology in the millimetre-wave band. For this reason, a comparison between gap waveguide and SIW technology in terms of conductor, dielectric and leakage losses at 170 $\mathrm{GHz}$ was performed. The advantages of the gap waveguide are explained, especially with the aim to reduce the leakage losses. Gap waveguide appears to be more flexible than SIW for the design in the millimetre-wave band, because it is less limited in the formulation to reduce leakage losses.

In Chapter 4, the feeding transition of the gap waveguide was presented. The proposed transition consists on a CPW-to-microstrip-to-H-shape aperture feeding the gap waveguide. This transition exhibits low losses and is very suitable for the integration in the same module for LTCC manufacturing process. Several prototypes of the gap waveguide have been manufactured with R04003 and LTCC. A good compromise between the simulated and measured results for RO4003 prototype was achieved. However, line impedance showed a strong sensitivity to tolerance errors in air gap height, in the gap waveguide. In turn, this sensitivity leads to mismatching in the transition. LTCC appears here, as a good candidate to overcome this limitation, because the process allows integrating cavities in the same co-fired process. Nevertheless, carrying out the internal cavity is one of the most complicated technical procedures with LTCC. For this reason, an exhaustive study in terms of design and manufacturing tests in the laboratory has been done. Results of this study are very promising, the final tests showed that we can control the shrinkage of the cavities, the pressure in lamination and the temperature profile for internal cavities embedded in LTCC tapes. With these results, we have manufactured the LTCC gap waveguide. Some technical problems appeared. One is via metallization that showed a bad contact between the LTCC layers. The other one is related to the internal cavities and the top metallic layer.

Chapter 5 presented the gap waveguide resonator. The resonator is based on a $3 N / 4$ open-stub placed on top of the vias added to the strip of the gap waveguide. Principal advantages of this resonator is that propagation takes place in the air gap, minimizing the losses at high frequency and also with the purpose of fluid 
characterization, a cavity or channel in this air gap produces maximum interaction between the fluid under test and the electromagnetic field. To manifest these advantages, a comparison with a structure based on Substrate Integrated Cavity was presented. Considering the same operating frequency, we compared the resonant frequency for three cases: empty cavity, methanol and water at $60 \mathrm{GHz}$. Results showed an increase in the sensitivity for gap waveguide due to the propagation in the maximum E-field level. However, in the case of SIC the interaction with the fluid is done by apertures placed in a ground plane connected with an external cavity. The other part of the chapter presented the results of the manufactured gap waveguide resonators, both created in the same prototypes explained in Chapter 4. Gap waveguide resonator using RO4003 exhibited very good performance, even with the limitation of the air gap. The manufacturing with LTCC suffered some issues related to the overflow of the metal inside the cavity and the via filling.

To sum up, this Ph.D. dissertation has proposed a new structure working in the millimetre-wave band that improve the sensibility for fluid characterization. Design considerations in terms of impedance, losses and comparison with other topologies have been presented. The design of the feeding transition and the manufacturing of both prototypes with PCB and LTCC have been shown. An important work in the LTCC laboratory with the aim to optimizing the manufacturing of internal and external cavities has been done. We propose with this thesis, expanding the range of frequency of the fluid characterization.

\section{Future lines}

There is a lot of work that can be done after this thesis. Firstly, the development of the gap waveguide using LTCC technology can be improved. With this purpose, in Chapter 4, several solutions were proposed to improve the LTCC manufacturing process.

Regarding the fluid characterization, next step consists on filling the microfluidic cavities with several liquids to practical measurements of the proposed sensor at $60 \mathrm{GHz}$. This point is very crucial in the complete validation of the gap waveguide for microfluidic applications. The validation with PCB and LTCC is expected to 
compare the performances and to show the biocompatibility of the different materials.

To explore other manufacturing techniques, we propose the use of PCB techniques combining with PDMS channels for the fluid introduction. In terms of manufacturing, this method is fast and simple, and it can be compatible also with the gap waveguide manufacturing.

For a further work, we propose also, other kind of resonators combining other manufacturing techniques as for example microfluidic chip based on liquid tunable transmission phase shifters. 


\section{APPENDIX A}

\section{LTCC process}

The unfired ceramic material is available in the form of sheets and it is referred to as green tape, (green color representing the unfired state of the ceramic). The process starts by defining the substrate size and number of layers. In LTCC fabrication, all the layers are processed parallel. Next, the design features such as channels, grooves and vias are created using techniques such as laser cutting, punching heads, jet-vapor etching and CNC machines. Conducting vias used as interconnects across layers are formed by filling the punched vias with conducting inks of $\mathrm{Au}$ or $\mathrm{Ag}$. Current lines and sensing/actuation electrodes are formed by screen printing. The laminated LTCC device is then sintered in a programmable furnace. The temperature ramping profile is a critical parameter during sintering with slow ramping rates recommended to avoid thermal stresses and shrinkage [81].

\section{Manufacturing steps}

In general, LTCC fabrication process is to build a multilayered substrate by making metallization and functional structures on several single layered dielectric glass/ceramic sheets and laminate them together and sintering in 850$900^{\circ} \mathrm{C}$ temperature. The fabrication process is shown in Figure 1. Manufacturing steps in LTCC. 
The first step of LTCC substrate manufacturing is green tape preparing procedure before blanking, that is mixing and casting. The second step is via punching, that it can be done with laser or mechanical method. Laser is better in accurate position, better quality via shape and smaller tape distortion. In the third and fourth step, punched vias are filled with conductive paste and then routing pattern is screen printed. For some microwave modules, cavities are needed. They are punched on single layers after screen printed patterns are heated and dry. After each single layer has been properly processed, they will be stacked and laminated to a green substrate.

After lamination, the green substrates are cut to proper size and are co-fired or sintered to "ceramic" substrates and then separated to the final size [79].

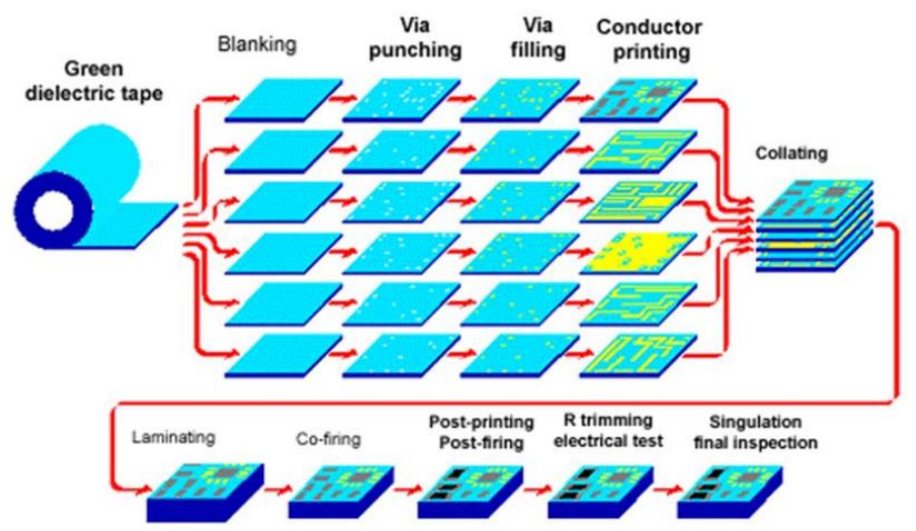

Figure 1. Manufacturing steps in LTCC. 


\section{APPENDIX B}

\section{LTCC process at TELECOM BRETAGNE}

The LTCC manufacturing process has been presented in Chapter 1. In this section, we are focused in the manufactured process using our equipment.

First of all, it is necessary to explain the layout processing for LTCC circuits (Figure 2). Basically, once the design using CAD software as for example ADS (Advanced Design System), two kinds of files are needed: files for photo-plotter and files for laser cutting. The files for photo-plotter consist on .hpgl files that allow the performance of the masks. Files for the laser are separated in files to create vias (.drl) and files to create complex shapes for the performance of cavities (.dxf). 


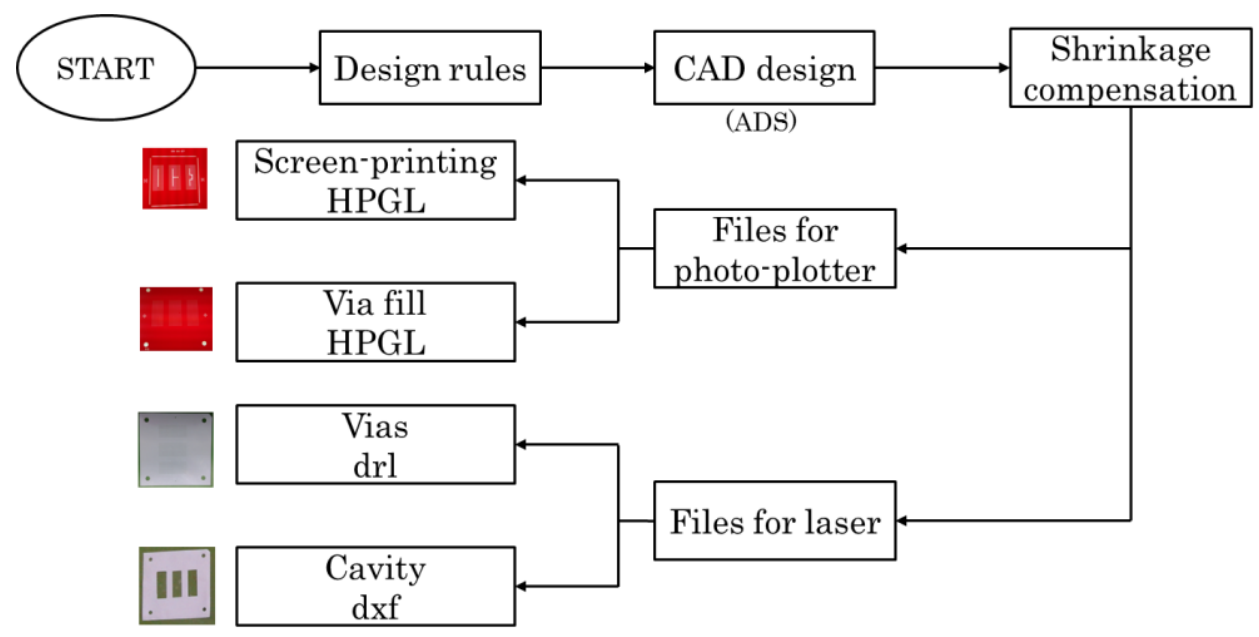

Figure 2.Schema for the layout processing for LTCC.

In the other hand, the steps to create prototypes with LTCC in our laboratory are:

\section{Slitting, blanking and preconditioning}

LTCC green tapes are shipped on a roll. The tapes are cut at the exact dimensions, in our case $50.8 \times 50.8 \mathrm{~mm}$. Next, preconditioning is useful to eliminate the stress induced by the removal of the backing tape. Depending on the material composition, the temperature is between $80^{\circ} \mathrm{C}$ and $120^{\circ} \mathrm{C}$, and the duration is between 15 and 30 minutes.

\section{Structure formation: vias and cavities}

Next step consist on the creation of the vias and cavities. Vias and cavities are conventionally formed by mechanical punching or laser machining. Mechanical punching offers high accuracy and good hole quality. Nevertheless, the minimum dimension of the diameter is limited by the size of the punching tools. Therefore, lasers offers la possibility to made complex shapes with rounded or curved edges for the cavity creation and laser machining allows the creation of different via diameters without changing drilling tools for each diameter.

Our laser machine is ASTREE 250 model from NovALSE society. It is based on the CO2 or Neodymium-doped Yttrium Aluminum Garnet (ND-YAG) laser. This laser is dedicated to the micro-cutting and micro-drilling of different composites 
and materials such as ceramic, plastic and metal. This laser is indeed a ND-YAG source with wavelength of $1064 \mathrm{~nm}$ and frequency between 20 and $100 \mathrm{kHz}$. The maximal output power is $16 \mathrm{~W}$ (at $100 \mathrm{kHz}$ ) and the spot size is around $50 \mu \mathrm{m}$. Tests were been performed to determine the laser parameters for each kind of LTCC tape, depending on the thickness, and the properties of the layer. With this laser, it is possible to create diameters of 150 until $400 \mu \mathrm{m}$.

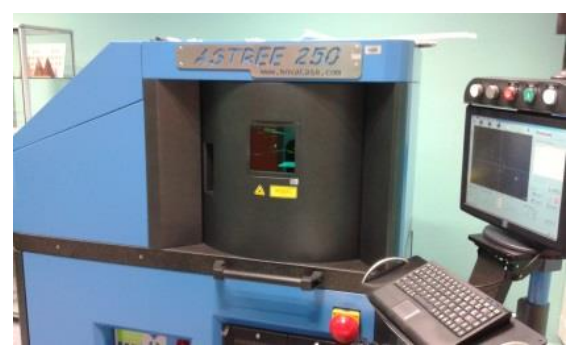

Figure 3. Laser machine AsTREE 250 model

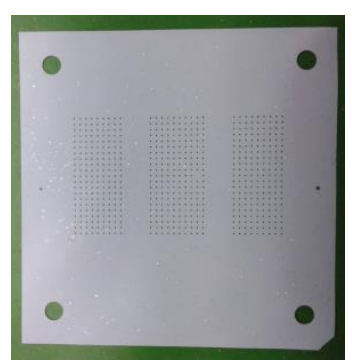

Figure 4. Example of drilled holes with laser machining

\section{Via fill}

The via holes are filled with the semi-automated thick film screen printer model CP-465 from PRESCO. The via fill paste is gold ESL802 through a 325 mesh screen.

\section{Screen printing}

Conductors on LTCC are implemented by screen printing method. Our screen printing method use the same screen printer PRESCO CP-465 used for via fill process. Screen with 325 and 400 mesh count are used to obtain fine printing resolutions. The photosensitive emulsion with $20 \mu \mathrm{m}$ of thickness is also used for fine conductor patterns.

\section{Stacking and alignment}

Next step consist on the stacking of all the LTCC layers to create a single block. Nowadays, they are two methods for alignment: manual by using pin alignment fixtures or automatic alignment with vision systems. In our laboratory, this process is done manually where each layer is stacked onto a fixture that has a set of pins that correspond to the registration holes of each layer (Figure 5). This fixture is composed of three pins instead of four pins, to minimize the 
stretching of the layers during stacking. A protecting material is used at the start and the end of stacking operation to avoid the contact of LTCC material with the metal plates during lamination.

Then, , after sealing the LTCC sheet corners, the LTCC block is placed under vacuum to evacuate the air located between the LTCC layers (Figure 6).

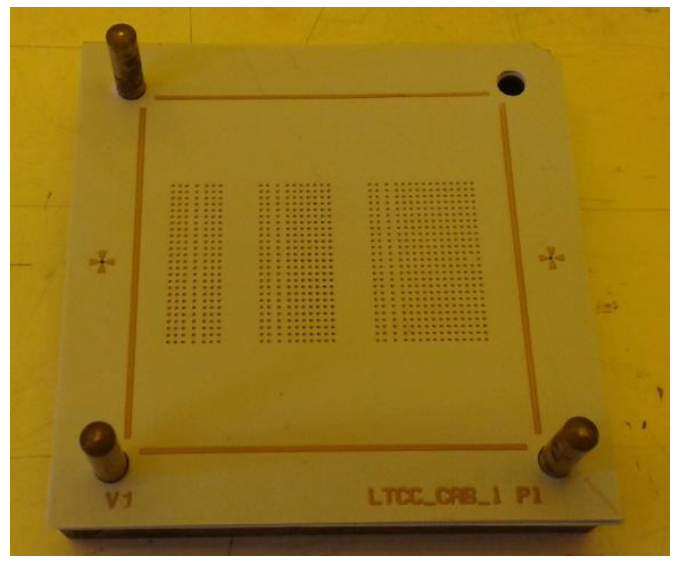

Figure 5. Stacking LTCC layers

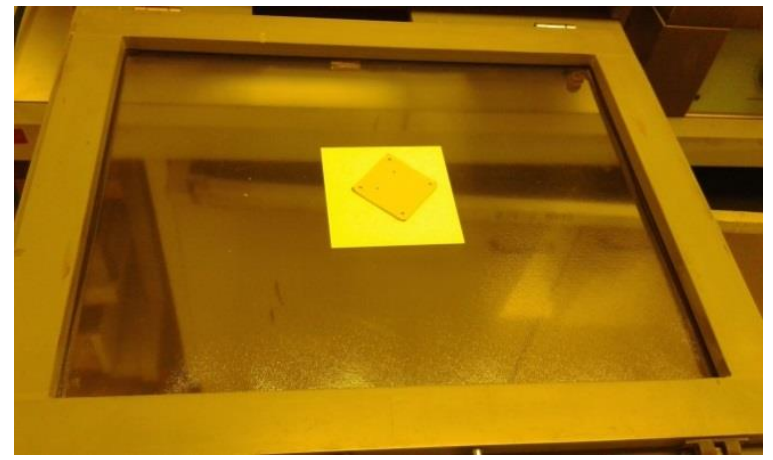

Figure 6. LTCC layer under vacuum to eliminate air between layers

\section{Lamination}

Once the layers are stacked, the LTCC block is laminated under heat and pressure to ensure a strong contact between layers and to avoid delamination. There are two possibilities for the LTCC lamination process: the uniaxial lamination and the isostatic method.

The first one, the uniaxial process, the tapes are pressed between heated plates at 200 bars, $70^{\circ} \mathrm{C}$ for 10 minutes approximately. This method is characterized by speed and simplicity. The uniaxial lamination could cause difficulties to create cavities and it can causes higher shrinkage than isostatic lamination. The second method uses an isostatic press. The stacked tapes are vacuum packaged in a foil and pressed in hot water. The LTCC substrate is pressed in hot water about 350 bars. 
Lamination process in our laboratory uses the uniaxial method as it is illustrated in Figure 7. The sacrificial volume material (SVM) inserted to avoid deformation in cavities in the lamination process is ESL49000.

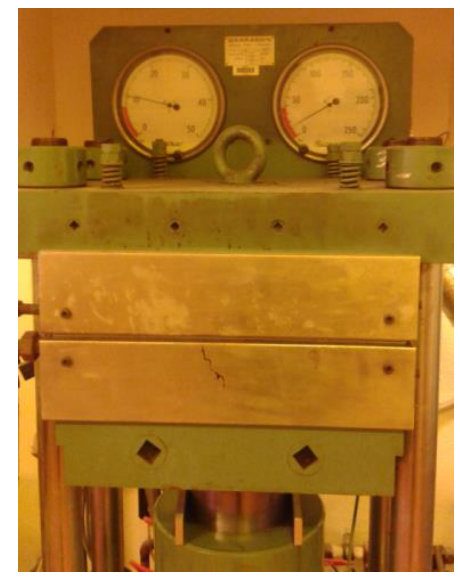

Figure 7. Uniaxial press machine for lamination process

\section{Co-firing}

Finally, the laminated structures are fired in a single block using a specific firing profile. During the firing cycle, the unfired LTCC substrate must be placed on a setter. The firing profile generally depends on the material composition; ad must be modified in the case of large and thick substrates. In figure $\mathrm{x}$, the furnace of our laboratory is presented and in Figure 8 the typical LTCC profile of temperature.

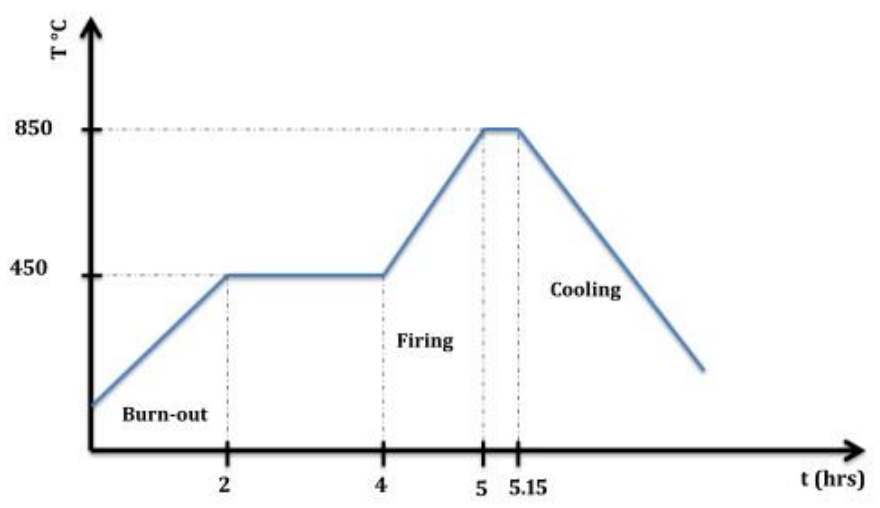

Figure 8. Temperature profile for LTCC process 


\section{APPENDIX C}

\section{Gap Waveguide manufacturing with $\mathrm{RO} 4003$}

The RO4003 device is composed of six gap waveguide prototypes. The layout of the Layout of the manufactured prototype:

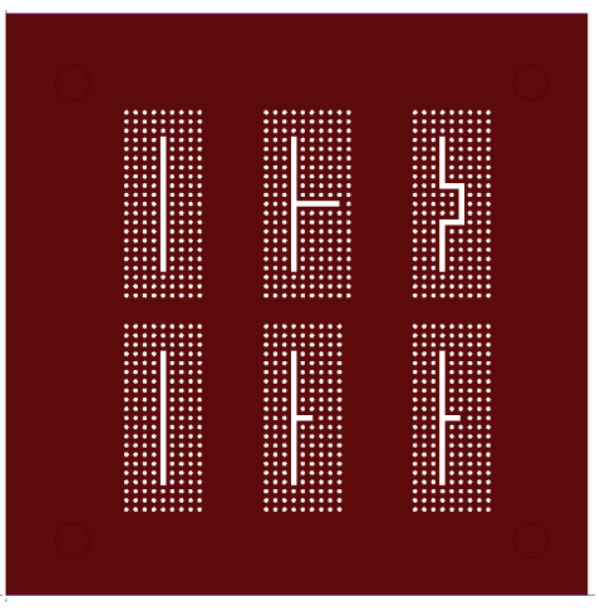

a) Layout of the siw gap waveguide prototypes

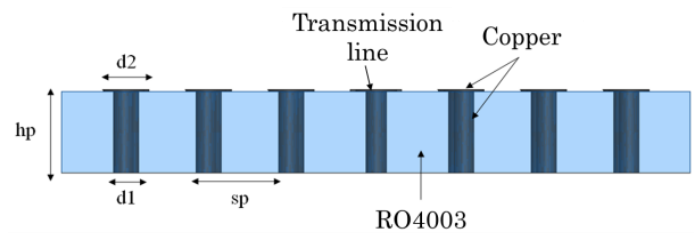

b) Front view of one gap waveguide prototype

Dimensions are specified in Table 1. 
Table 1. Dimensions of the RO4003 gap waveguide prototype.

\begin{tabular}{|c|c|c|}
\hline \multicolumn{2}{|c|}{ Dimension } & Value \\
\hline $\mathrm{hp}$ & $\begin{array}{c}\text { Pin height (substrate } \\
\text { thickness) }\end{array}$ & $813 \mu \mathrm{m}$ \\
\hline $\mathrm{D} 1$ & Pin diameter & $200 \mu \mathrm{m}$ \\
\hline $\mathrm{D} 2$ & Patch diameter & $400 \mu \mathrm{m}$ \\
\hline $\mathrm{Sp}$ & Pins periodicity & $800 \mu \mathrm{m}$ \\
\hline $\mathrm{wr}$ & Width of the transmission line & $500 \mu \mathrm{m}$ \\
\hline
\end{tabular}

The coplanar-to-microstrip-to-H-shape aperture transition was manufactured in a separate process. The dimensions and layout is shown in

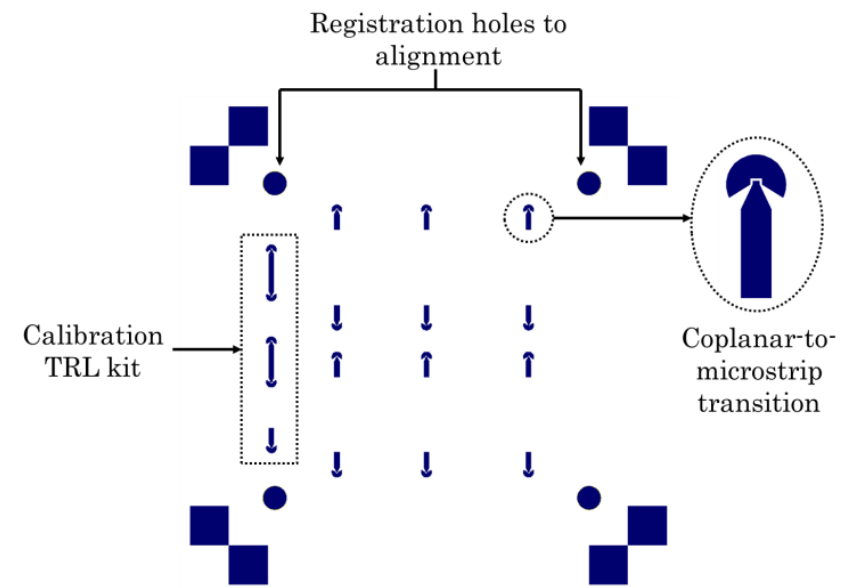

a) Layout of the transition performed with ADS solver.

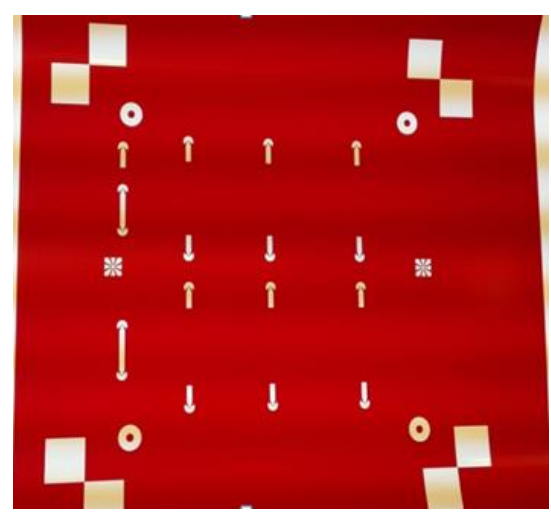

b) Film mask

Figure 9. Coplanar-to-microstrip transition (top view). 


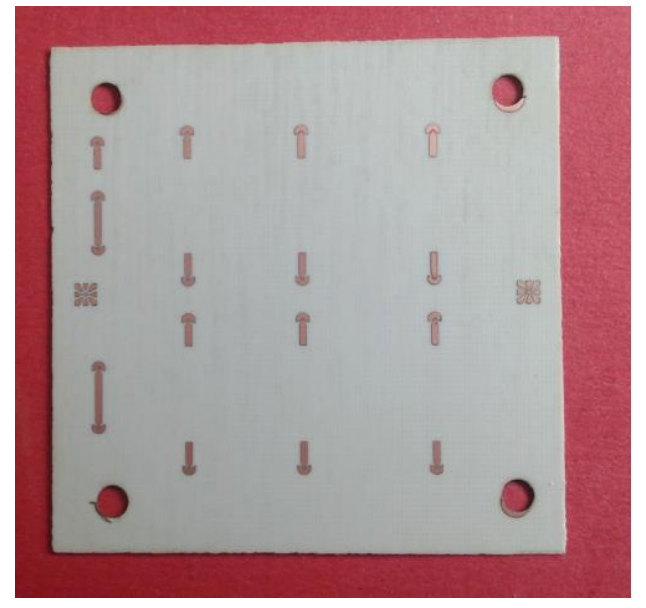

Figure 10. Manufactured CPW-to-microstrip transition with RO4003.

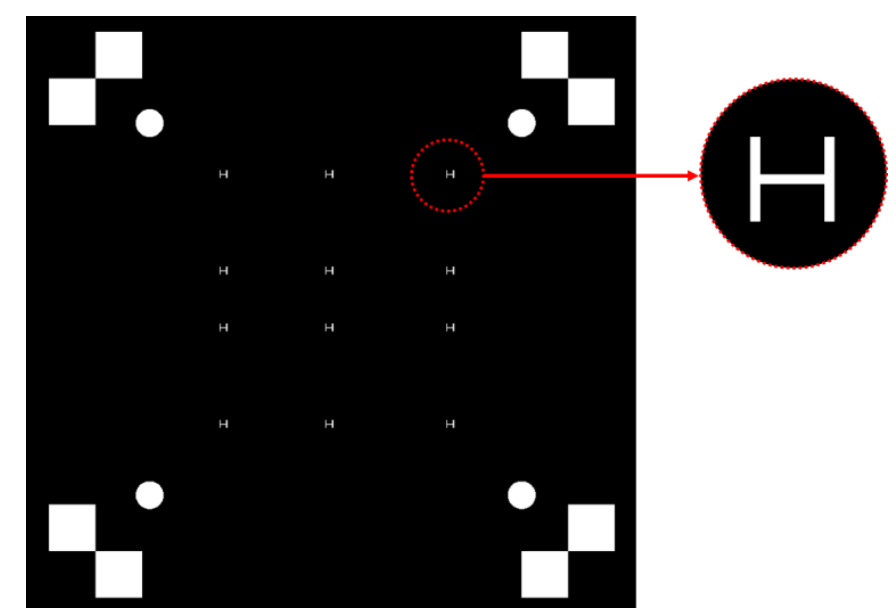

Figure 11. Layout of the ground plane with the $\mathrm{H}$-shape apertures (bottom layer).

A $508 \mu \mathrm{m}$ thickness R04003 substrate thickness has been cut with the laser to create a square base between transition and gap waveguide forming the air cavity.

First, the three parts forming the structures have been placed in a metallic base to make first measurements. 

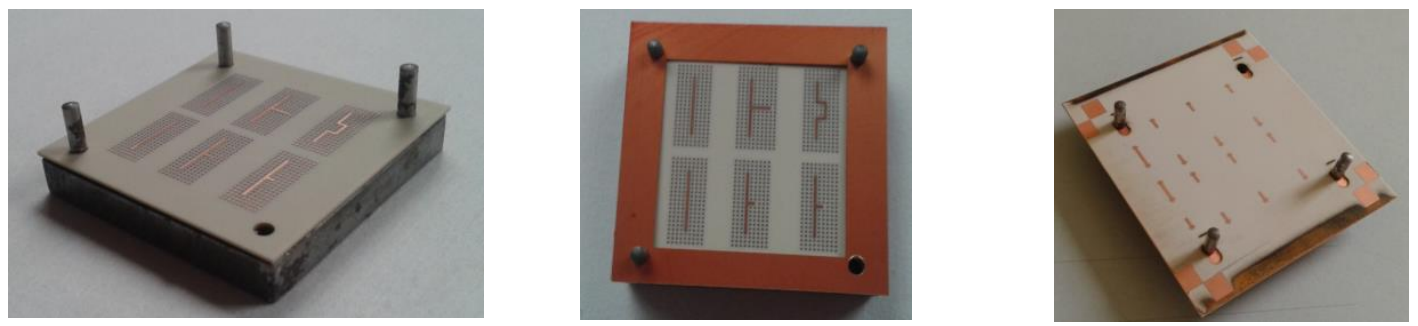

\section{Measurement information}

To better accuracy in the results, two calibrations have been done, one with the calibration kit designed in the own RO4003 prototype and second, with a TRL calibration kit standard.

a) Measurements of the back to back CPW-MSL with calibration kit designed in the prototype

Back to back CPW to microstrip line
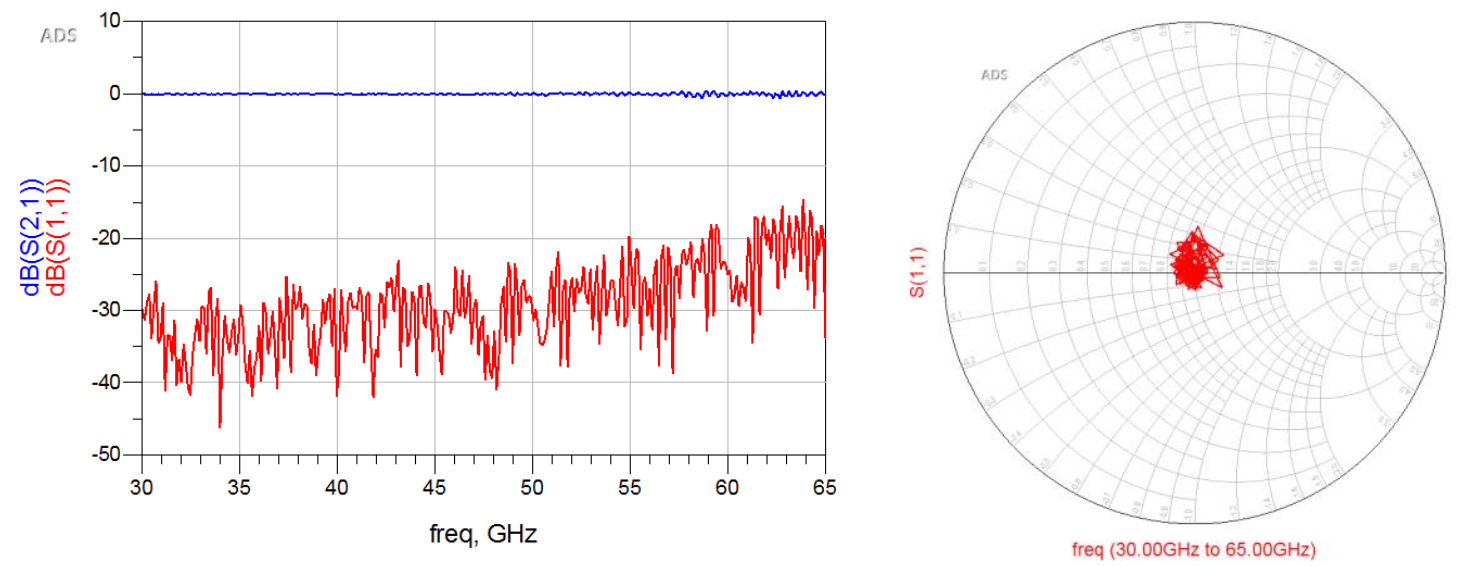

b) Measurement of the back to back CPW-MSL with the calibration kit standard 

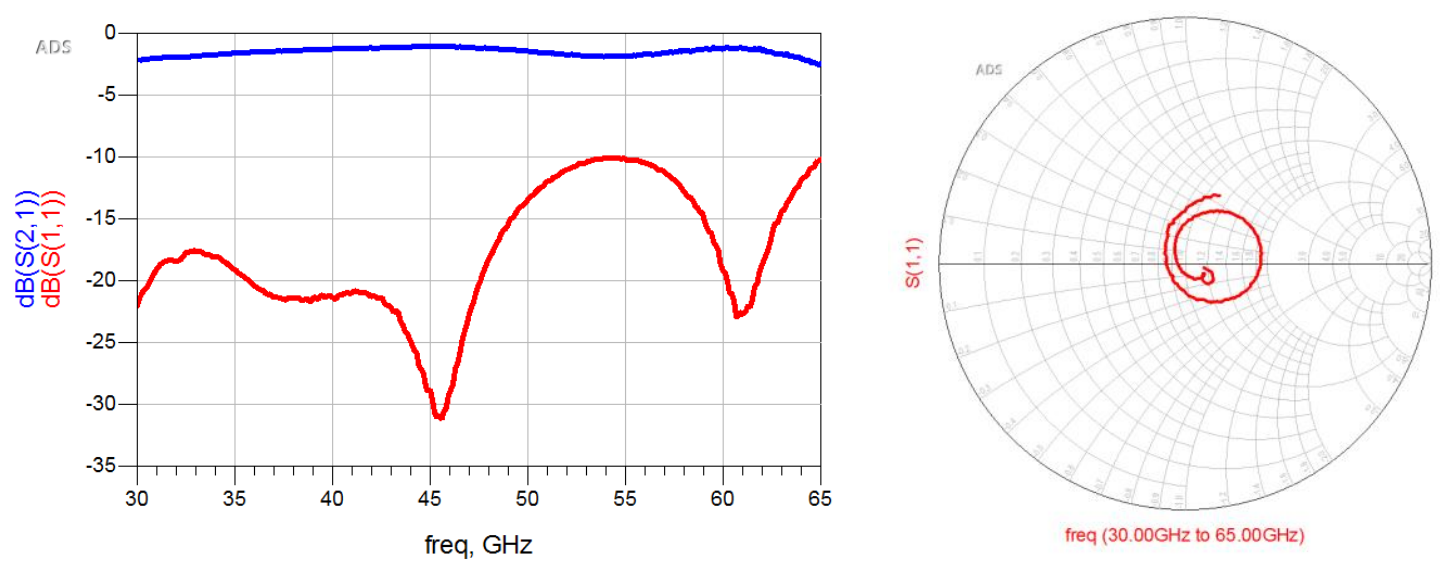

It is normal this difference, because in the last case, calibration has been done using and external kit, and measurements are referenced respect to this measurements. On the other hand in the first case, as the calibration kit is the same transition CPW-MSL measured after, S21 in the band is $0 \mathrm{~dB}$. 


\section{BIBLIOGRAPHY}

[1] B. R. Jean, E. C. Green, and M. J. McClung, "A microwave frequency sensor for non-invasive blood-glucose measurement," in IEEE Sensors Applications Symposium, 2008. SAS 2008, 2008, pp. 4-7.

[2] D. Stuerga, "Microwave-Material Interactions and Dielectric Properties, Key Ingredients for Mastery of Chemical Microwave Processes," in Microwaves in Organic Synthesis, A. Loupy, Ed. Wiley-VCH Verlag GmbH, 2006, pp. 161.

[3] T. Chen, D. Dubuc, M. Poupot, J. Fournie, and K. Grenier, "Accurate Nanoliter Liquid Characterization $\mathrm{Up}$ to $40 \mathrm{GHz}$ for Biomedical Applications: Toward Noninvasive Living Cells Monitoring," IEEE Trans. Microw. Theory Tech., vol. 60, no. 12, pp. 4171-4177, Dec. 2012.

[4] E. Topsakal, T. Karacolak, and E. C. Moreland, "Glucose-dependent dielectric properties of blood plasma," in General Assembly and Scientific Symposium, 2011 XXXth URSI, 2011, pp. 1-4.

[5] K. Shibata and M. Kobayashi, "Measurement of complex permittivity for liquids using the coaxial line reflection method," in 2015 Asia-Pacific Symposium on Electromagnetic Compatibility (APEMC), 2015, pp. 452-455.

[6] T. P. Marsland and S. Evans, "Dielectric measurements with an open-ended coaxial probe," Microw. Antennas Propag. IEE Proc. H, vol. 134, no. 4, pp. 341-349, Aug. 1987.

[7] P. M. Meaney, A. P. Gregory, N. R. Epstein, and K. D. Paulsen, "Microwave open-ended coaxial dielectric probe: interpretation of the sensing volume revisited," BMC Med. Phys., vol. 14, p. 3, Jun. 2014.

[8] T. S. R Zajíček, "Medical Diagnostics Using Reflection Method and Waveguide Probes - Feasibility Study."

[9] K. Shibata, "Measurement of Complex Permittivity for Liquid Phantom by Transmission Line Method Using Coaxial Line," IEICE Trans. Electron., vol. E87-C, no. No.5, pp. 689-693, 2004. 
[10] P. Vainikainen, "Method for accurate measurement of complex permittivity of tissue equivalent liquids," Electron. Lett., vol. 36, no. 1, pp. 32-34, Jan. 2000

[11] "Measurement of Dielectric Material Properties - Application Note - RAC0607-0019."

[12] B.-K. Chung, "Dielectric constant measurement for thin material at microwave frequencies," Prog. Electromagn. Res.-Pier - PROG ELECTROMAGN RES, vol. 75, pp. 239-252, 2007.

[13] J. M. Catala-Civera, A. J. Canos, F. L. Penaranda-Foix, and E. de los Reyes Davo, "Accurate determination of the complex permittivity of materials with transmission reflection measurements in partially filled rectangular waveguides," IEEE Trans. Microw. Theory Tech., vol. 51, no. 1, pp. 16-24, Jan. 2003.

[14] J. C. Booth, N. D. Orloff, J. Mateu, M. Janezic, M. Rinehart, and J. A. Beall, "Quantitative Permittivity Measurements of Nanoliter Liquid Volumes in Microfluidic Channels to $40 \mathrm{GHz}$," IEEE Trans. Instrum. Meas., vol. 59, no. 12, pp. 3279-3288, Dec. 2010.

[15] J. Roelvink, S. Trabelsi, and S. O. Nelson, "A Planar Transmission-Line Sensor for Measuring the Microwave Permittivity of Liquid and Semisolid Biological Materials," IEEE Trans. Instrum. Meas., vol. 62, no. 11, pp. 29742982, Nov. 2013.

[16] B. Kapilevich, A. Yahalom, B. Litvak, and D. Mihaeli, "Microwave diagnostics of some bio-medical solutions using cylindrical resonator," in IEEE EUROCON 2009, EUROCON 'O9, 2009, pp. 128-133.

[17] M. Neshat, H. Chen, S. Gigoyan, D. Saeedkia, and S. Safavi-Naeini, "Whispering-gallery-mode resonance sensor for dielectric sensing of drug tablets," Meas. Sci. Technol., vol. 21, p. 015202, Jan. 2010.

[18] A. Taeb, S. Gigoyan, G. Rafi, S. Safavi-Naeini, and M. Neshat, "A low cost and sensitive sensor based on the Whispering Gallery Mode at D-band," in Microwave Conference (EuMC), 2011 41st European, 2011, pp. 619-622.

[19] C. Liu and Y. Pu, "A Microstrip Resonator With Slotted Ground Plane for Complex Permittivity Measurements of Liquids," IEEE Microw. Wirel. Compon. Lett., vol. 18, no. 4, pp. 257-259, Apr. 2008.

[20] T. Chretiennot, D. Dubuc, and K. Grenier, "A Microwave and Microfluidic Planar Resonator for Efficient and Accurate Complex Permittivity Characterization of Aqueous Solutions," IEEE Trans. Microw. Theory Tech., vol. 61, no. 2, pp. 972-978, Feb. 2013.

[21] N. Meyne, G. Fuge, H. K. Trieu, A.-P. Zeng, and A. F. Jacob, "Miniaturized Transmission-Line Sensor for Broadband Dielectric Characterization of Biological Liquids and Cell Suspensions," IEEE Trans. Microw. Theory Tech., vol. 63, no. 10, pp. 3026-3033, Oct. 2015.

[22] S. Pinon, D. L. Diedhiou, A.-M. Gué, N. Fabre, G. Prigent, V. Conédéra, E. Rius, C. Quendo, B. Potelon, J.-F. Favennec, and A. Boukabache, "Development of a microsystem based on a microfluidic network to tune and reconfigure RF circuits," J. Micromechanics Microengineering, vol. 22, no. 7, p. article id. 074005, Jul. 2012. 
[23] W. R. McGrath, C. Walker, M. Yap, and Y.-C. Tai, "Silicon micromachined waveguides for millimeter-wave and submillimeter-wave frequencies," IEEE Microw. Guid. Wave Lett., vol. 3, no. 3, pp. 61-63, Mar. 1993.

[24] H. Shigesawa, M. Tsuji, and A. A. Oliner, "Simultaneous propagation of bound and leaky dominant modes on printed-circuit lines: a new general effect," in Microwave Symposium Digest, 1995., IEEE MTT-S International, 1995, pp. 145-148 vol.1.

[25] M. Bozzi, A. Georgiadis, and K. Wu, "Review of substrate-integrated waveguide circuits and antennas," IET Microw. Antennas Propag., vol. 5, no. 8, pp. 909-920, Jun. 2011.

[26] N. Ranjkesh and M. Shahabadi, Loss Mechanisms in Siw and Msiw. .

[27] P.-S. Kildal, E. Alfonso, A. Valero-Nogueira, and E. Rajo-Iglesias, "Local Metamaterial-Based Waveguides in Gaps Between Parallel Metal Plates," IEEE Antennas Wirel. Propag. Lett., vol. 8, pp. 84-87, 2009.

[28] F. E. H. Tay, Microfluidics and BioMEMS Applications. Springer Science \& Business Media, 2013.

[29] L. L. Wu, L. A. Marshall, S. Babikian, C. M. Han, J. G. Santiago, and M. Bachman, "A printed circuit board based microfluidic system for point-ofcare diagnostics applications."

[30] C. Iliescu, H. Taylor, M. Avram, J. Miao, and S. Franssila, "A practical guide for the fabrication of microfluidic devices using glass and silicon," Biomicrofluidics, vol. 6, no. 1, pp. 016505-016505-16, Mar. 2012.

[31] J.-C. Souriau, L. Castagne, G. Parat, P. Nicolas, P.-L. Charvet, G. Simon, K. Amara, P. D'hiver, B. Boutaud, and R. Dal Molin, "Characterization of a hermetic silicon box fitted in a cardiac lead in order to measure the endocardial acceleration signal," in Electronics System-Integration Technology Conference (ESTC), 2014, 2014, pp. 1-5.

[32] J. R. Anderson, D. T. Chiu, R. J. Jackman, O. Cherniavskaya, J. C. McDonald, H. Wu, S. H. Whitesides, and G. M. Whitesides, "Fabrication of topologically complex three-dimensional microfluidic systems in PDMS by rapid prototyping," Anal. Chem., vol. 72, no. 14, pp. 3158-3164, Jul. 2000.

[33] M. R. Gongora-Rubio, P. Espinoza-Vallejos, L. Sola-Laguna, and J. J. Santiago-Avilés, "Overview of low temperature co-fired ceramics tape technology for meso-system technology (MsST)," Sens. Actuators Phys., vol. 89, no. 3, pp. 222-241, avril 2001.

[34] J. Luo, "The Development and Biocompatibility of Low Temperature CoFired Ceramic (LTCC) for Microfluidic and Biosensor Applications," Theses Diss.--Chem. Mater. Eng., Jan. 2014.

[35] K. A. Peterson, R. T. Knudson, E. J. Garcia, K. D. Patel, M. Okandan, C. K. Ho, C. D. James, S. B. Rohde, B. R. Rohrer, F. Smith, L. R. Zawicki, and B. D. Wroblewski, "LTCC in microelectronics, microsystems, and sensors," in 15th International Conference on Mixed Design of Integrated Circuits and Systems, 2008. MIXDES 2008, 2008, pp. 23-37.

[36] K. A. Peterson, K. D. Patel, C. K. Ho, S. B. Rohde, C. D. Nordquist, C. A. Walker, B. D. Wroblewski, and M. Okandan, "Novel Microsystem 
Applications with New Techniques in Low-Temperature Co-Fired Ceramics," Int. J. Appl. Ceram. Technol., vol. 2, no. 5, pp. 345-363, Sep. 2005.

[37] S. Löffler, G. Reppe, A. Rebs, M. Herrmann, C. Mauermann, A. Kaiser, and K.-H. Fritz, "Thickfilm ceramic ready for MCM Interposer," European Microelectronics Packaging Conference (EMPC), Friedrichshafen, Germany, Sep-2015.

[38] P.-S. Kildal, E. Alfonso, A. Valero-Nogueira, and E. Rajo-Iglesias, "Local Metamaterial-Based Waveguides in Gaps Between Parallel Metal Plates," IEEE Antennas Wirel. Propag. Lett., vol. 8, pp. 84-87, 2009.

[39] P.-S. Kildal, "Artificially soft and hard surfaces in electromagnetics," IEEE Trans. Antennas Propag., vol. 38, no. 10, pp. 1537-1544, Oct. 1990.

[40] A. Polemi, S. Maci, and P.- . Kildal, "Dispersion Characteristics of a Metamaterial-Based Parallel-Plate Ridge Gap Waveguide Realized by Bed of Nails," IEEE Trans. Antennas Propag., vol. 59, no. 3, pp. 904-913, Mar. 2011.

[41] P.-S. Kildal, "Three metamaterial-based gap waveguides between parallel metal plates for $\mathrm{mm} / \mathrm{submm}$ waves," in 3rd European Conference on Antennas and Propagation, 2009. EuCAP 2009, 2009, pp. 28-32.

[42] A. Valero-Nogueira, E. Alfonso, J. I. Herranz, and P.-S. Kildal, "Experimental Demonstration of Local Quasi-TEM Gap Modes in SingleHard-Wall Waveguides," IEEE Microw. Wirel. Compon. Lett., vol. 19, no. 9, pp. 536-538, Sep. 2009.

[43] E. Rajo-Iglesias and P.-S. Kildal, "Groove gap waveguide: A rectangular waveguide between contactless metal plates enabled by parallel-plate cutoff," in 2010 Proceedings of the Fourth European Conference on Antennas and Propagation (EuCAP), 2010, pp. 1-4.

[44] A. Valero-Nogueira, M. Baquero, J. I. Herranz, J. Domenech, E. Alfonso, and A. Vila, "Gap Waveguides Using a Suspended Strip on a Bed of Nails," IEEE Antennas Wirel. Propag. Lett., vol. 10, pp. 1006-1009, 2011.

[45] D. Sievenpiper, L. Zhang, R. F. J. Broas, N. G. Alexopolous, and E. Yablonovitch, "High-impedance electromagnetic surfaces with a forbidden frequency band," IEEE Trans. Microw. Theory Tech., vol. 47, no. 11, pp. 2059-2074, Nov. 1999.

[46] H. Raza, J. Yang, P.-S. Kildal, and E. Alfonso Alos, "Microstrip-Ridge Gap Waveguide \#x2013;Study of Losses, Bends, and Transition to WR-15," IEEE Trans. Microw. Theory Tech., vol. 62, no. 9, pp. 1943-1952, Sep. 2014.

[47] E. Rajo-Iglesias, A. U. Zaman, E. Alfonso, and P.-S. Kildal, "Alternative ridge gap waveguide design using a mushroom-type EBG surface," in IEEE Antennas and Propagation Society International Symposium, 2009. APSURSI 'O9, 2009, pp. 1-4.

[48] E. Rajo-Iglesias and P.-S. Kildal, "Numerical studies of bandwidth of parallel-plate cut-off realised by a bed of nails, corrugations and mushroomtype electromagnetic bandgap for use in gap waveguides," IET Microw. Antennas Propag., vol. 5, no. 3, pp. 282-289, Feb. 2011.

[49] M. Bosiljevac, Z. Sipus, and P.-S. Kildal, "Construction of Green's functions of parallel plates with periodic texture with application to gap waveguides - 
a plane-wave spectral-domain approach," IET Microw. Antennas Propag., vol. 4, no. 11, pp. 1799-1810, Nov. 2010.

[50] E. Alfonso, P.-S. Kildal, A. Valero-Nogueira, and M. Baquero, "Study of the characteristic impedance of a ridge gap waveguide," in IEEE Antennas and Propagation Society International Symposium, 2009. APSURSI '09, 2009, pp. $1-4$.

[51] A. Polemi and S. Maci, "Closed form expressions for the modal dispersion equations and for the characteristic impedance of a metamaterial-based gap waveguide," IET Microw. Antennas Propag., vol. 4, no. 8, pp. 1073-1080, août 2010.

[52] E. Alfonso, M. Baquero, A. Valero-Nogueira, J. I. Herranz, and P.-S. Kildal, "Power divider in ridge gap waveguide technology," in 2010 Proceedings of the Fourth European Conference on Antennas and Propagation (EuCAP), 2010, pp. 1-4.

[53] E. Pucci, A. U. Zaman, E. Rajo-Iglesias, P.-S. Kildal, and A. Kishk, "Losses in ridge gap waveguide compared with rectangular waveguides and microstrip transmission lines," in 2010 Proceedings of the Fourth European Conference on Antennas and Propagation (EuCAP), 2010, pp. 1-4.

[54] E. Pucci, P.-S. Kildal, and E. Rajo-Iglesias, "Evaluation of losses in microstrip gap waveguide for slot antennas applications," in 2012 IEEE Antennas and Propagation Society International Symposium (APSURSI), 2012, pp. 1-2.

[55] E. Pucci, A. U. Zaman, E. Rajo-Iglesias, P.-S. Kildal, and A. Kishk, "Study of $\mathrm{Q}$-factors of ridge and groove gap waveguide resonators," IET Microw. Antennas Propag., vol. 7, no. 11, pp. 900-908, Aug. 2013.

[56] K. H. RIDA, "Packaging of Microwave Integrated Circuits in LTCC Technology," Thesis, Télécom Bretagne, Université de Bretagne Occidentale, 2013.

[57] R. Garg, I. J. Bahl, and M. Bozzi, Microstrip lines and slotlines. Boston, MA: Artech House, 2013.

[58] J. Xu, Z. N. Chen, X. Qing, and W. Hong, "140-GHz SIW LTCC antenna array using a large via-fenced and slotted dielectric loading," in 2013 7th European Conference on Antennas and Propagation (EuCAP), 2013, pp. 2861-2864.

[59] E. Pucci, A. U. Zaman, E. Rajo-Iglesias, P.-S. Kildal, and A. Kishk, "Losses in ridge gap waveguide compared with rectangular waveguides and microstrip transmission lines," in 2010 Proceedings of the Fourth European Conference on Antennas and Propagation (EuCAP), 2010, pp. 1-4.

[60] H. Raza, J. Yang, P.-S. Kildal, and E. Alfonso Alos, "Microstrip-Ridge Gap Waveguide \#x2013;Study of Losses, Bends, and Transition to WR-15," IEEE Trans. Microw. Theory Tech., vol. 62, no. 9, pp. 1943-1952, Sep. 2014.

[61] F. Shigeki, "Waveguide line (in Japanese)," Japan Patent 06-053 711, 25Feb-1994.

[62] D. Deslandes and K. Wu, "Accurate modeling, wave mechanisms, and design considerations of a substrate integrated waveguide," IEEE Trans. Microw. Theory Tech., vol. 54, no. 6, pp. 2516-2526, Jun. 2006. 
[63] M. Bozzi, A. Georgiadis, and K. Wu, "Review of substrate-integrated waveguide circuits and antennas," IET Microw. Antennas Propag., vol. 5, no. 8, p. 909, 2011.

[64] D. Deslandes and K. Wu, "Design Consideration and Performance Analysis of Substrate Integrated Waveguide Components," in Microwave Conference, 2002. 32nd European, 2002, pp. 1-4.

[65] Y. Cassivi, L. Perregrini, P. Arcioni, M. Bressan, K. Wu, and G. Conciauro, "Dispersion characteristics of substrate integrated rectangular waveguide," IEEE Microw. Wirel. Compon. Lett., vol. 12, no. 9, pp. 333-335, Sep. 2002.

[66] F. Xu and K. Wu, "Guided-wave and leakage characteristics of substrate integrated waveguide," IEEE Trans. Microw. Theory Tech., vol. 53, no. 1, pp. 66-73, Jan. 2005.

[67] M. Pasian, M. Bozzi, and L. Perregrini, "A Formula for Radiation Loss in Substrate Integrated Waveguide," IEEE Trans. Microw. Theory Tech., vol. 62 , no. 10, pp. 2205-2213, Oct. 2014.

[68] M. Bozzi, M. Pasian, L. Perregrini, and K. Wu, "On the losses in substrate integrated waveguides," in Microwave Conference, 2007. European, 2007, pp. 384-387.

[69] M. Bozzi, F. Xu, D. Deslandes, and K. Wu, "Modeling and Design Considerations for Substrate Integrated Waveguide Circuits and Components," in 8th International Conference on Telecommunications in Modern Satellite, Cable and Broadcasting Services, 2007. TELSIKS 2007, 2007, p. P-VII-P-XVI.

[70] C.-H. Jung, S.-H. Lim, and N.-H. Myung, "Analysis of an H-shape cross slotted aperture-coupled microstrip patch antenna," in Microwave Conference, 2009. APMC 2009. Asia Pacific, 2009, pp. 1890-1893.

[71] E. Pucci, E. Rajo-Iglesias, and P.-S. Kildal, "New Microstrip Gap Waveguide on Mushroom-Type EBG for Packaging of Microwave Components," IEEE Microw. Wirel. Compon. Lett., vol. 22, no. 3, pp. 129-131, Mar. 2012.

[72] C. Arenas Buendia, F. Gallee, A. Valero-Nogueira, and C. Person, "Gap waveguide structure in LTCC for millimeter-wave applications," in 2014 8th European Conference on Antennas and Propagation (EuCAP), 2014, pp. 982-986.

[73] E. Alfonso, P.-S. Kildal, A. Valero-Nogueira, and M. Baquero, "Study of the characteristic impedance of a ridge gap waveguide," in IEEE Antennas and Propagation Society International Symposium, 2009. APSURSI '09, 2009, pp. 1-4.

[74] H. Raza, J. Yang, P.-S. Kildal, and E. Alfonso Alos, "Microstrip-Ridge Gap Waveguide \#x2013;Study of Losses, Bends, and Transition to WR-15," IEEE Trans. Microw. Theory Tech., vol. 62, no. 9, pp. 1943-1952, Sep. 2014.

[75] H. Wang, B. Cao, Y. Huang, and J. Zheng, "High-Gain L-Probe Excited Substrate Integrated Cavity Antenna Array with LTCC-based Gap Waveguide Feeding Network for W-band Application," IEEE Trans. Antennas Propag., vol. PP, no. 99, pp. 1-1, 2015. 
[76] B. Kapilevich, A. Yahalom, B. Litvak, and D. Mihaeli, "Microwave diagnostics of some bio-medical solutions using cylindrical resonator," in IEEE EUROCON 2009, EUROCON 'O9, 2009, pp. 128-133.

[77] T. Chen, D. Dubuc, M. Poupot, J. Fournie, and K. Grenier, "Accurate Nanoliter Liquid Characterization $\mathrm{Up}$ to $40 \mathrm{GHz}$ for Biomedical Applications: Toward Noninvasive Living Cells Monitoring," IEEE Trans. Microw. Theory Tech., vol. 60, no. 12, pp. 4171-4177, Dec. 2012.

[78] P.-S. Kildal, E. Alfonso, A. Valero-Nogueira, and E. Rajo-Iglesias, "Local Metamaterial-Based Waveguides in Gaps Between Parallel Metal Plates," IEEE Antennas Wirel. Propag. Lett., vol. 8, pp. 84-87, 2009.

[79] C. Arenas Buendia, F. Gallee, A. Valero-Nogueira, and C. Person, "Gap waveguide structure in LTCC for millimeter-wave applications," in 2014 8th European Conference on Antennas and Propagation (EuCAP), 2014, pp. 982-986.

[80] S. Liu, I. Ocket, D. Schreurs, B. Nauwelaers, and W. De Raedt, "A $60 \mathrm{GHz}$ liquid sensing substrate integrated cavity in LTCC," in Microwave Conference (EuMC), 2013 European, 2013, pp. 613-615.

[81] A. Vasudev, A. Kaushik, K. Jones, and S. Bhansali, "Prospects of low temperature co-fired ceramic (LTCC) based microfluidic systems for point-ofcare biosensing and environmental sensing," Microfluid. Nanofluidics, vol. 14, no. 3-4, pp. 683-702, Nov. 2012.

[82] I. Wolff, "Design and Technology of Microwave and Millimeterwave LTCC Circuits and Systems," in International Symposium on Signals, Systems and Electronics, 2007. ISSSE' '07, 2007, pp. 505-512. 


\section{RELATED PUBLICATIONS}

\section{International conferences}

1. Cristina Arenas-Buendia, Francois Gallee, Alejandro Valero Nogueria, Christian Person, "Gap Waveguide Structure in LTCC for MillimeterWave Applications", Antennas and Propagation (EuCAP),2014 8th European Conference, April 2014.

2. Cristina Arenas-Buendia, François Gallée, Alejandro Valero Nogueira, Christian Person, "RF Sensor based on Gap Waveguide technology in LTCC for liquid sensing", Antennas and Propagation (EuCAP), 2015 9th European Conference, April 2015.

3. Cristina Arenas-Buendia, François Gallée, Alejandro Valero Nogueira, Christian Person, «LTCC Technology for Microfluidic Applications based on the Gap Waveguide Technology ", European Microelectronics and Packaging Conference (EMPC), September 2015.

\section{National conferences}

1. Cristina Arenas-Buendia, François Gallee, Alejandro Valero Nogueira, Christian Person, "Estructura Gap Waveguide en Tecnología LTCC para aplicaciones sub-milimétricas", URSI 2013, XXVIII Simposium Nacional 2013 Santiago de Compostela (Spain).

2. Cristina Arenas-Buendia, Francois Gallee, Alejandro Valero Nogueria, Christian Person, "Microfluidic application based on Gap Waveguide 
Topology in the Millimeter-Wave band with LTCC technology", URSI 2014, XXIX Simposium Nacional, 2014 Valencia (Spain).

3. Cristina Arenas-Buendia, François Gallée, Alejandro Valero Nogueira, Christian Person, "Caractérisation des milieux fluidiques à partir de structures basées sur la topologie "Gap Waveguide”, Journées Nationales de Micro־ondes, Juin 2015

\section{Workshops}

1. GapWave Workshop Chalmers November 2013 Feasibility of realizing gap waveguides in LTCC technology.

2. Lab-STICC Workshop (Poster presentation), 11 July 2014

3. Journées Ecole Doctorale SICMA, 25 September 2014

Best oral presentation award

4. LTCC Technology for microfluidic millimetre-wave applications, $3^{\text {rd }}$ Advanced Technology Workshop on Microelectronics November 2015, Lyon.

\section{Co-autor}

1. Camilla Kärnfelt, Jean-Philippe Coupez, Pascal Coant, Cristina ArenasBuendia, François Gallée, Daniel Bourreau, Alain Péden, Khodor Rida, "Le développement de la technologie LTCC dans le LabSticc pour une intégration mixte RF et optoélectronique", Journée du Club UMR FOTON Lannion 19 Juin 2014. 